Nijmeegse Geografische Cahiers nr. 40

\title{
Small Business Growth in Contrasting Environments
}

Peter Vaessen 

small business growth in contrasting environments 
Vaessen, Peter Mathias Maria.

Small Business Growth in Contrasting Environments / P.M M Vaessen. - Nijmegen: Department of Social Geography, Faculty of Admunistrative Sciences, Catholic University of Nijmegen. - I11 , fig., tab. - (Nijmeegse geografische cahiers ; no 40)

Also publ. in commercial ed : Utrecht : Royal Dutch Geographic Socicty ; Nijmegen : Faculty of Administrative Sciences, Catholic University of Nijmegen, 1993. - Netherlands geographical studies, ISSN 0169-4839; 165). Thesis Nijmegen. - With ref.

ISBN 90-70898-38-1

Subject headings: small business growth.

ISBN 90-70898-38-1 (Nijmeegse geografische cahiers 40)

ISBN 90-6809-178-6 (Netherlands geographical studies 165)

Copyright (C) 1993 Catholic University of Nijmegen, The Netherlands

Alle rechten voorbehouden. Niets uit deze uitgave mag worden vermenigvuldigd en/of openbaar gemaakt door middel van druk, fotokopie of op welke andere wijze dan ook zonder voorafgaande toestemming van de auteur.

All rights reserved. No part of this publication may be reproduced in any form, by print or photoprint, microfilm or any other means, without written permission from the authors.

Drukkerij Elinkwijk bv - Utrecht 


\title{
small business growth in contrasting environments
}

een wetenschappelijke proeve op het gebied van de beleidswetenschappen

\author{
proefschrift
}

ter verkrijging van de graad van doctor aan de Katholieke Universiteit Nijmegen, volgens besluit van het College van Decanen in het openbaar te verdedigen op vrijdag 1 oktober 1993 des ochtends te 10.30 uur precies

door

peter mathias maria vaessen

geboren op 19 mei 1955 te nijswiller - wittem 
promotor: prof. dr. egbert wever 
CONTENTS

List of figures 7

List of tables 7

Acknowledgements 8

1 Introduction 9

1.1 Trends in the space economy 9

1.2 The main thrust: agglomeration and inequality 10

1.3 The actual trajectories: deviancy and contradiction 11

1.4 Research question and scope of the book 15

2 Firm and environment in regional science 17

2.1 Introduction 17

2.2 The primacy of the environment 17

2.3 The primacy of the enterprise 24

2.4 Linking environmental and organizational approaches 34

2.5 Conclusion 40

3 Organization and environment; the perspective of organization theory 43

3.1 Introduction 43

3.2 The environment 44

3.3 Three perspectives on action 55

3.4 The role of organizational size 70

3.5 Summary 77

$4 \quad$ Research method and sample 81

4.1 Introduction 81

4.2 A qualitative method 81

4.3 The sample 92

$5 \quad$ Environmental responsiveness of small firms (I): its forms 97

5.1 Introduction 97

5.2 Coping with the labour market 98

5.3 Dealing with the financial environment 115

5.4 Managing the material input environment 120

5.5 Handling the business service environment 127

5.6 Meeting the market environment 136

5.7 Coping with the environment; summary and conclusions 147 
6 Environmental responsiveness of small firms (II): its strength 151
6.1 Introduction 153
6.2 Adaptation costs 152
6.3. Adaptation returns 154
6.4 The sustainability of revenues from adaptation behaviour 160
6.5 Summary 164

7 Environmental responsiveness of small firms (III): its origins 167

7.1 Introduction 167

7.2 The regional ecology 168

7.3 The incubator organization 171

7.4 Success and failure $\mathbf{1 7 4}$

7.5 Summary and conclusions $\mathbf{1 7 5}$

$8 \quad$ Summary and conclusions 179
8.1 Summary 179
8.2 Conclusions 185

Bibliography 189

Appendix 205

Korte Nederlandse samenvatting 217 
2.1 Substitution of low locational by extra economic or adaptation efficiency 28
2.2 The fallacy of the substitution framework 32

3.1 Levels in the environment according to McDermott and Taylor 44

3.2 Levels in the environment according to Castrogiovanni 45

3.3 Two conceptualizations of the administrative environment 49

3.4 The regional component in an administrative model of the environment 53

3.5 The regional component built into Castrogiovanni's enviromental model 53

3.6 Adaptive abilities, state of adaptation, and adaptive fits 76

4.1 The Netherlands; the location of the firms investigated 95

\section{Tables}

3.1 Dimensions and subdimensions of the environment 48

3.2 Means of dealing with the external environment 66

5.1 The spatial scope of the sample firms' sales linkages in 1988143 


\section{Acknowledgements}

A number of people have provided considerable help towards the conducting of the research on which this thesis is based, and the preparation of the text. Foremost I thank my promotor Prof. Dr. E. Wever for his stimulating support and valuable advice. He created a very stimulating work climate by giving me sufficient elbowroom while at the same time showing great interest in the work during the course of the project. I am also indebted to the entrepreneurs interviewed, for their willing cooperation with the present research. This is particularly true as the extensive interviews have demanded much of their time and effort. Furthermore, I thank the staff members of the Department of Social and Economic Geography of the University of Nijmegen for forming a pleasant work environment, for their stimulating comments, and for any assistence when needed. In addition I thank the commission consisting of Prof. Dr. J. Buursink, Prof. Dr. J.G. Lambooy and Dr. D. Keeble who evaluated the thesis.

Thanks are also due, with regard to the preparation of the manuscript. I acknowledge very much the services of drs. Nancy Smyth van Weesep who corrected the text with respect to stylistic and linguistic errors. I am also very grateful to my wife drs. Paula Roovers for preparing the lay out, the tables and the figures. Apart from that she did take over the finishing of the manuscript and dealt with the manuscript commission during my stay abroad at the University of Cambridge (UK) between April and August 1993. I am also in debt to my wife's younger sister Nathalie Roovers for coming to my help when the situation was hectic. Moreover, I am obliged to Willem Maas and Allen Swindell for some translation work. Finally, I am very thankful to Dr. Keeble for making a final check on the manuscript for detecting and correcting remaining typing, spelling and grammatical errors. 


\section{Chapter One}

\section{Introduction}

\section{$1.1 \quad$ Trends in the space economy}

Reality is complex. In order to understand something, we must abstract from it. Academics tend to do this by generalizing from numerous observations. Social scientists often search for trends and express these in models. They are more concerned with the dominant processes and phenomena than with the exceptions, the deviations from the 'norm'. So too regional economists and economic geographers. Scott's account of the main trends in geographic development since the beginning of the 1950s, is revealing. In the mid 1950s, the major capitalist economies seemed to be split into two spatial realms. One was a core of manufacturing regions with dense concentrations of population and economic activity. The other consisted of peripheral expanses of less well developed territory, largely dedicated to agriculture and local service activities, with occasional clusters of industrial employment in a few favoured places (Scott 1988a). Furthermore, many theorists considered the contrasts between the core and the periphery to be extremely durable and likely to intensify (Hirschman 1958, Myrdal 1957).

During the 1970s, however, this pattern began to shift dramatically. As the postwar boom came to an end, the major capitalist economies (North America and Western Europe) entered a long period of crisis and readjustment which continues to the present day. More and more of the basic industry of the core regions - steel, shipbuilding, car assembly, machinery, electrical goods, and so on - have moved to the world periphery. There they can take advantage of increasingly attractive production conditions (particularly low wages and regressive labour relations).

The net result of these developments is a major crisis in the core regions of North America and Western Europe. The crisis first became apparent in Britain and the United States in the late 1960s, spreading in the early 1970 s to France, Italy, and West Germany. In many formerly prosperous industrial regions, investment in new plants and machinery has slowed to a trickle. Large units of fixed capital are written off in an epidemic of factory closures and layoffs. In the United States, several major cities are at the brink of bankruptcy, as their tax bases shrink in response to their declining economies.

Nevertheless, even as these processes of decay and decline reach their greatest intensity, some regions in advanced capitalist nations are moving counter to the general trend. They are actually experiencing a rapid formation of new manufacturing 
capital and a rapid rise in employment. These exceptions comprise either (a) a number of enclaves within older manufacturing regions (e.g., Route 128, The Scientific City Ile de France, the M4 Corridor), or (b) more importantly, a series of regions located in formerly peripheral and semi-peripheral areas (e.g., Third Italy, Orange County, Silicon Valley, Grenoble, Montpellier, Sophia Antipolis, Toulouse, Cambridge) (Scott 1988b). By the mid-1980s it was clear that these regions had not only weathered the storm of restructuring of the 1970s but were also functioning as major foci of industrial innovation and expansion in their own right. It was now quite evident that a series of new industrial spaces had come in existence, and were beginning to form important alternative centres of capitalist accumulation. This process was based on a strong social division of labour, the proliferation of small to medium-sized businesses, and the reagglomeration of production. According to Scott, reagglomeration occurs at all geographic levels. A new combination of economic activities occurs on the international and national scale; this also takes place at the regional level, particularly in new growth areas.

The above overview sketches in rough contours, the geographic pattern over the industrial development of the past three to four decades, as Scott sees it. There is, however, one development which Scott tends to neglect: the come back of many large cities and core regions in the 1980s. Their economic perspectives improved either because of a revival of existing industries (e.g., Swiss watchmaking) or because of the boom the service sector is enjoying. Services have been concentrated in large cities all along, either in the city centre or in the urban ring. In the 1970s and 1980s, the intensity of concentration actually increased (Storper and Walker 1989). In sum, the geographic development of Western Capitalism since the beginning of the industrial revolution can be classified in three successive stages: concentration, decentralization, and reconcentration.

\subsection{The main thrust: agglomeration and inequality}

The three above-mentioned processes have formed the focal point of geographic studies and the object of model-building. In the course of time, the concentration and inequality model has come to predominate. Spatial proximity of business ventures and facilities generates major economic advantages, it is argued (the next chapter expands on this topic). Geographic polarization is viewed as a process of spatial optimization. This model posits that tight clustering in large cities and (new) industrial areas is the optimal location pattern for many economic activities. Consequently, international and interregional inequality of growth is seen as an inevitable concomitant and condition of growth itself (Hansen 1970). This is held to be true even during the decentralization period of the 1960s and 1970s. Many researchers point out that in this period decentralisation was not an integral and complete fanning-out process (Crum and Gudgin 1977, 150; Goddard 1978). Businesses only decentralized their routine production activities. To be sure, much employment also moved in the decentralization process, but these were large blue-collar jobs or requiring a low level of skills. Ultimately this trend held back the development of provincial centres. The research departments as well as the management stayed behind in the large cities. 
These eschelons were (and are) thought to be bound to a highly educated labour market, tight information flows and old-boys networks. In other words, the swarming-out process did not fundamentally challenge the core-periphery model of economic growth. And indeed, many countries, both Western and non-Western reveal marked differences between thriving core areas and depressed peripheral areas. The distribution of industry, generally speaking, is still rather clustered than dispersed (Chapman and Walker 1991). On the whole, agglomeration and regional inequality comprise what Abler (1991) terms "the laws of economic geography".

\subsection{The actual trajectories: deviancy and contradiction}

However, the spatial structures are not as unambiguous as the above model would suggest. Differences exist between countries, sectors, and activities. In a (small) country like the Netherlands, for example, it is difficult to observe a strong reconcentration of production in one or a few favoured growth areas in the aftermath of decentralization. After a comprehensive analysis of the economic geographical developments in our country, De Smidt and Wever (1990) conclude that the traditional core-periphery model is being dismantled. This is evident from in the spread of promising industries, the magnitude of innovation in the diverse regions, the receptivity to innovation stimuli, the location of industrial head offices, the geography of Philips plants, etc. It is increasingly clear that the differences within The Netherlands are only minor. De Smidt $(1988,126)$ concludes that the distribution of affluence within regions is more extreme than between regions. A similar picture emerges from an analysis of the spatial pattern of business vitality ${ }^{1}$ in the Netherlands by Tordoir and co workers (TNO 1992). Among other things they observed that areas of above and below average business vitality sometimes lie very close to each other.

Gaebe (1988) reaches the same conclusion regarding indicators of regional development in West Germany. The differences in demographic and economic characteristics are greater within the member states than between them. In the United States, Kasarda (1988) concludes that since 1983 there has been a convergence of the interregional differences in employment opportunities between the Northeast, the Midwest, the South and the West. Nevertheless, the South and the West will continue to lead population and employment growth until the turn of the century at the least. Anyway, Kasarda is more worried about the development of intraregional than interregional differences. In particular, he points out that the contrast within metropolitan areas between the central core and the suburban ring only increases the prosperity of the latter. In England, on the other hand, researchers concern themselves mainly with differences in development at the interregional level. They are especially interested in the growing split between the problematic North and the prosperous South (Harrison and Hart 1992).

But the level of aggregation of these outcomes is high. Thus, the figures mask the difference between many contradictory developments and processes. The overall picture is often made up of a mix of concentration and decentralization tendencies, and possibly a substantial portion of spatial arbitrariness. For example, take the spatial figures in the Netherlands. On several fronts, there is a flow out of the major 
agglomerations of the Randstad towards less urbanized areas, such as the southern and eastern areas of the country This flow consists of business movements (Pellenbarg and Kemper 1991), high-technology industry (Louter 1991), and general employment growth (Lambooy and Manshanden 1992) Other factors work to the advantage of the Randstad In this area we see an overrepresentation of foreign investments (Loeve 1989, Buck 1988), head offices (De Smidt and Wever 1990), high-quality business services (Louter 1992), and new business establishments (Kemper 1992, Bleichrodt and Louter 1991) According to Kemper, closure rates are remarkably similar across space However, Wever (1984), Bleumink et al (1985), Vaessen (1986), and Gordijn and Van Wissen (1992) report very high death rates in the major Dutch cities, whereby their advantage of high birth rates vanishes into thin air This has also been observed in London (Peters 1991)

The spatial pattern is strongly differentiated for a phenomenon such as business vitality (TNO 1992) In this regard the picture is varied and runs counter to every model of regional development Kleınknecht and Poot's (1992) 'multi-dimensional' conclusion regarding the spatial pattern of R\&D activities of private businesses is also very useful for demonstrating the diversity and ambiguity of spatial processes in the Netherlands Kleinknecht and Poot find little evidence that firms in centralized regions engage more often in $R \& D$ or undertake relatively greater amounts of $R \& D$ than similar firms in other regions Yet, it appears that industrial firms in the central region (the Randstad) are more intensively involved in product and service-related research, while industrial firms located elsewhere are more deeply involved in development activities (though the differences are not large) At first sight, this image of higher quality of industrial R\&D activities in the Randstad compared with the rest of the country seems to get reinforced by the spatial pattern of the service sector Service firms in the four major cities are more R\&D intensive than those outside those cities However, contrary to indications in manufacturing, service firms in the Randstad appear not to be more involved with fundamental and applied research in comparison with service firms located elsewhere In short, "The question whether regions matter for R\&D cannot be answered with a simple 'yes' or 'no'" (Kleinknecht and Poot 1992, 230) Both a 'no' and a 'yes' may be justified

Outside the Netherlands, the diversity of geographic development is hardly less, as some simple examples demonstrate In England, spatial trends are unmistakably dominated by the divide between the deprived northern and the prosperous southern part Yet some figures do not fit this picture at all For instance, variation in new firm formation does not conform to the core-penphery model of economic performance. Northern Ireland, Wales and Scotland show higher rates of job creation than the UK average, together with the South East, the Midlands and East England Below-average regions are the South West, the North, Yorkshire and Humberside, the North West and the West Midlands (Peters 1990,12) Another example which runs counter to the picture of the North-South divide in Great Britain is the spatial variation of new biotechnology firms This sector forms an indicator of economic development perspectives These firms are very dispersed spatially, Oakey and Cooper (1989) note that no less than $75 \%$ of the new biotechnology firms are located in rural and peripheral areas Concerning the geography of all high-tech development, Keeble $(1988,80)$ concludes that ' the geography of high-technology development in the $1980 \mathrm{~s}$ is one of widely dispersed growth in most regions of Britain, but with a particular focus on a 
broad band of southern English counties...". In any case, high-tech activities are in no way restricted to one or a few so-called 'high-profile' areas.

Keeble's conclusion fits the results of research by Oakey and O'Farrell (1991) concerning state-of-the-art production technologies in the form of computer numerically controlled ( $\mathrm{CNC}$ ) machinery to ensure high quality output at a competitive price. Oakey and $\mathrm{O}^{\prime}$ Farrell compare the adoption of CNC equipment in the core (Southeast England) with two peripheral regions (Scotland and Wales). The differences seem minor; surprisingly, there is a (slightly) deeper commitment to CNC machinery in Scotland and Wales (presumably a regional policy effect). Analysis of the growth performance of high technology companies runs even more counter to the prevailing North-South picture within the United Kingdom than analysis of the location of these activities. Keeble (1993) made recently some very interesting observations in this respect. He noted that though much fewer in number, NTBFs [New Technology Based Firms] established in 'hostile' environments often achieve high - and indeed higher - growth rates than their counterparts in more favourable environments. NTBFs in the three peripheral and old industrial regions of Scotland, Northern England and Wales reported percentage employment and turnover growth rates 1987-90 which were on average substantially greater than those for NTBFs in southern England, with employment growth three times faster than in London, and turnover growth five times faster than in the South East outside London. Furthermore, he added that this difference cannot be explained by size differences ${ }^{2}$ since Peripheral NTBFs are in fact on average larger than those in any other region (ibid 7).

In Germany, just as in England, biotechnology firms and information technology activities are widely dispersed at the interregional level, though there is a particular concentration in Baden Würtemberg. However, in contrast with the British Isles, the spatial pattern largely follows the urban hierarchy at the intraregional level (i.e. within the federal states). These activities are concentrated respectively in urban areas 95.1\% and 93.7\% (Henckel 1989). Thus, the the overall spatial pattern is polycentric. The strong German Mittelstant enterprises, on the other hand, seem to operate much less in large cities. These small and mid-size companies, which are at key to Germany's export success, work out of small plants often located in towns most people have never heard of (Business Week 1991). Observing such diversity, Gaebe states that opinions about a region's development potential just depend on the size of the areas studied and the indicators used. It is thus no surprise that the descriptions of regional development in Germany differ. Some observe pronounced disparities while others see a more balanced growth (Gaebe 1988, 179).

Unlike Britain and Germany, France displays strong concentration of both hightech establishments and employment. The preferred locations are the Ile de France and the 'Grand Sud'. However, France's spatial dynamics are less clear-cut. The number of establishments in the field of high-tech consulting remained highly concentrated between 1976 and 1987. Meanwhile, a substantial decentralization of employment opportunities occurred in this period (Moulaert et al. 1991).

Furthermore, no matter how emphatically research results confirm the agglomeration imperative, researchers are continually confronted with exceptions to the general rule and deviations from the norm which challenge conventional logic. Take, for example, electrotechnical and instrument industry in Great Britain. In accordance 
with a priori expectations, most firms in these branches are located in areas of concentrated economic activity. Nevertheless, a considerable minority (25\%) are located in rural and peripheral areas (Oakey and Cooper 1989). The same emerged from Oakey's, Rothwell's and Cooper's comparison of R\&D expenditure by firms in the San Francisco Bay Area (including Silicon Valley), Southeast England, and Scotland (Oakey et al 1988). As 'expected', firms in the core areas - the Bay Area and Southeast England - are more R\&D-intensive (respectively $91.7 \%$ and $85.0 \%$ of the firms conduct internal R\&D) than in Scotland $(77.8 \%)$, a problem area. At the same time, these figures clearly reveal that firms with $R \& D$ activities are not restricted to 'high quality areas' such as Southeast England and Silicon Valley. Small independent firms in peripheral areas can also develop technological innovations.

And even if percentages are quite small, we should not ignore the absolute number that can be included. Although only a minority of six per cent of West Germany's information technology and biotechnology firms is located in rural areas, it still comprises about 250 firms. Similarly, Appold (1991) reports that industrial research laboratories in the United States tend to locate within metropolitan counties $(90 \%)$. The remainder locate in rural counties. However, this $10 \%$ encompasses no less than 1055 establishments. No matter how much support we find for the assumption that new and innovative firms are concentrated in the centre or at the top of the urban and regional hierarchy, it is not a hard and fast rule.

The consequences of 'deviant cases' for spatial theory might be more farreaching than their quantity suggests. In fact, every successful firm located outside of the key centres of development can provide information about the conditional nature of the agglomeration imperative assumed in spatial theory. In theory-forming, the question of whether the spatial structure of the economy is determined by centralizing or decentralizing factors should be replaced by a query about the conditions under which clustering or dispersal occurs.

Apart from scientific arguments, a close analysis of 'paradoxical' cases, e.g. profitable enterprises in peripheral areas, can open up new perspectives for regional policy. Policymakers could learn from counterfactual businesses which have shown an ability to maintain their productivity and competitiveness in 'unfavourable' production environments. That may be more productive than putting all the effort into improving external conditions. Malecki and Nijkamp (1988) point out that it is impossible to offer compensation in peripheral areas for the wide range of comparative advantages in large urban core areas.

\subsection{Research question and scope of the book}

In sum, this thesis takes the variety of spatial developments and patterns as its point of departure. In doing so, it breaks with the tradition in geographical studies to analyse the dominant or prevailing developments. This means that the aim of the book is to find a starting point for an analysis of how similar firms develop in different environments; that is in economic core regions as well as economic periphery regions. It thus responds to Mason and Harrison's call for further research (1985, 4-5): "...there does remain a need for more detailed studies of the formation process 
and the role and growth of new and small firms in contrasting regional environments "

In this vein, the general research question of this thesis asks What is the underlying logic whereby some firms in the periphery are no less competitive than their counterparts in the centre? During the field-work, this broad research question gradually unfolded into three more specific questions

1 How do prospective firms respond to economic conditions in the production environment, both opportunities and limitations?

2 What can be said about the strength or efficacy of environmental responsiveness of small firms?

3 What are the ultımate sources underlyıng the firms' environmental responsiveness?

The structure of the book is as follows After this introduction, Chapter Two critically evaluates the views on firm environment interaction as propounded in spatial theories Its main point of criticism is the inferior or inadequate role of the micro-level, $1 \mathrm{e}$ the entrepreneur and his firm, in mainstream theory of regional science Chapter Three proceeds to examine the relationship between a furm and its environment in another field of science which concerns itself with this subject, 1 e organization theory In a sense, organization theory takes an opposite tack to regional science, as the former tends to overestimate the role of the organization or its management Yet we keep in mind that organization theory has recently been changing in this respect The chapter examines how we can utilize insights from organization theory in the fields of regional economics and economic geography in order to meet the objections against spatial theory, set out in Chapter Two Chapter Four explains the methodological aspects of the research Chapter Five goes on to extensively describe and interpret actual firm behaviour in relation to the characteristics of the regional production environment (research question number one) This chapter is based on the material from our own in-depth research among successful small and medium-sized businesses Contrary to what is implied by mainstream regional development theory, entrepreneurs do not resign themselves to the whims of the production environment They not only exploit the opportunities of the local production environment but also counteract regional shortcomings and disadvantages However, defiance of the environment often involves additional investments and expenditures, which send production costs upward With reference to this, Chapter $S_{1 x}$ poses the question whether, in the end, strategies to overcome environmental contingencies really can be of use, especially when competitors in more benign environments appear to be free of such investment The firms investigated suggest that the answer to this question nevertheless might be affirmative The chapter puts forth some arguments for this It elaborates on the effectiveness of environmental resistance strategies (research quest1on number two) Chapter Seven attempts to identify the possible fundamental sources of the businesses' ability to grow and resist external contingencies (research question number three) Chapter Eight, finally, summarizes the findings and puts forward some implications for policy and research 
Notes

1. Business vitality is measured in this TNO study on the basis of real turnover growth, increase of investments and net profit growth between 1982 and 1991)

2. Very small firms can sometimes achieve high percentage growth rates simply because of the low base level from which calculation is made. 


\section{Chapter Two}

\section{Firm and environment in regional science}

\section{$2.1 \quad$ Introduction}

An analysis of theories in regional science with respect to the relationship between (business) organization and environment bnings to light two perspectives The first infers the behaviour and functions of organizations from the environment in which they are located The second reasons more or less in the opposite way, the environment in which the firm locates is seen as a result of the firm's behaviour and actuvities The first emphasizes the study of the environment as such, especially the urban or regional environment From this perspective, the spatial environment is of overriding importance for the formation, behaviour, and performance of organizations Consequently, little if any leeway is left for the firm to influence the environment in which it is located The second perspective presupposes a much greater freedom of choice for organizations It pays close attention to the decisions and organizational structures and the influence these have on the spatial environment This school of thought views furms and organizations as area orgamzing mstttutions These two alternatives can be referred to respectively as the environmental versus the organizational approach The enviroumental perspective is also referred to as an ecological view (cf Lambooy 1984) The organizational perspective represents the whole micro level, pointing to the effects of both organizational structure and entrepreneurial or management behaviour Accordingly, it may also be called the behavioural approach in this thesis The present chapter examines both perspectives in greater detail and will evaluate them critically (section 22 and 23)

The dichotomy explotted in this exercise may suggest that regional science is strongly divided Yet several theories contain the seeds of convergence of the environmental and organizational approaches Section 24 discusses several ways in which the environmental and organizational logic may be linked to each other

22

The primacy of the environment

The supposition that the actions (including the choice of location) and performance of a firm are heavily affected by the attributes of the environment in which it is located forms the intuitive basis of mainstream thinking in regional science However, in the course of time, the impact of the environment has been assigned different forms in 
various spatial theories. We begin by discussing two schools of grand theory in which the spatial environment conditions business behaviour and performance. These are traditional location theory and modern regional development theory.

\subsubsection{Location theory}

The focus of traditional (i.e., classical and neo-classical) location theory is the singleplant firm. According to conventional location theory the behaviour of a (single-plant) firm is determined by a strong urge to occupy the best or so-called optimal location. That is to say, an entrepreneur's location decision is guided by either spatial differences in total production costs or by spatial differences in total revenue (or demand), or by both. It should be added, however, that traditional location theory has been preoccupied with the cost side of production rather than with the demand side. According to Smith (1987, 27), this is partly due to the greater conceptual and practical difficulty of dealing with demand and revenue.

In any case, classical location theory, with its roots in the work of Weber, is centred on the firm's search for the location where production costs are lowest (i.e., least-cost location). There is only one least-cost location for a firm. The theory assumes that the cost of acquiring the quantity of a particular input necessary for production of a certain volume of output differs from place to place (i.e., the spatial cost surface). Furthermore, it is assumed that spatial cost surfaces differ according to the input material. All individual input cost surfaces add up to a surface of total costs, from which the least-cost location can easily be derived.

A major shortcoming of variable cost theory is its neglect of demand considerations. Hotelling, Lösch and others changed this situation. The least-cost location became counterbalanced by the maximum-revenue location. It is assumed that (locational) behaviour of firms is most strongly determined by revenue or demand conditions, which vary from place to place. This may be due to spatial differences in price (for example because of varying distribution and production costs). But it may also be due to the number of consumers and their characteristics, such as income, taste and preferences. Thus, "Revenue also becomes a continuous spatial variable, analogous to the total-cost surface, with one point at which total revenue will be maximized" (Smith 1987, 28-29).

Smith (1966) brings together the least-cost approach and the maximumrevenue approach in a single model. According to Smith, the optimal location for a firm is the location where the difference between costs and revenues is greatest i.e. the maximum-profit location. Apart from the optimal location, there are second-best areas in which revenues exceed costs. On these locations, production is profitable as well, though profits are not maximal. Smith refers to this as the spatial margin to profitability.

In traditional location theory, either spatial profitability differentials between firms or firms' locational behaviour are assumed to be completely determined by locational cost and demand conditions. Furthermore, the incorporation of nonoptimizing behaviour in location theory (by introducing the concept of spatial margin) creates more latitude for making location decisions. However, this does not apply to 
business performance, because it is assumed that every deviance from the 'optimal location' is accompanied by a proportional decrease in profitability. Consequently, the spatial margin concept is just as spatially deterministic as the rest of traditional location theory.

\subsubsection{Regional development theory}

In the early 1950s, both classical location theory (Weber) and its neoclassical version (Lösch, Hotelling, Isard, Smith and others) started to attract criticism. This criticism came from urban and regional economists and geographers. Regional inequality was, in reality, much greater than could be explained with a plant-by-plant analysis. Various economic activities showed a striking and stubborn inclination to cluster in large cities and urban areas. The economic landscape was perceived to consist of fastgrowing, densily populated urban core areas on the one hand and lagging, sparsely populated peripheral areas on the other. This conspicuous regional split was the basis on which various regional development theories were elaborated. Examples are Friedman's (1966) centre-periphery model, Myrdal's (1957) cumulative causation theory, Perroux's growth-pole theory (1950/1970, 1988), Vernon's (1966) product lifecycle theory, the filtering-down hypothesis (Lonsdale and Seyler 1979), and, recently, the geography of flexible specialisation (Scott 1983, 1988a, Scott and Storper 1987, Piore and Sabel 1984, Schoenberger 1988). All of these examples emphasize the quality of the environment as a factor in the fortunes of economic activities by employing the concept of agglomeration advantages. "The idea is that, spatial propinquity allows better access to the inputs and outputs necessary for production, thereby raising revenues, lowering costs, and increasing productivity" (Walker 1986, 248).

In this theory, urban areas offer noticeably better conditions for production than thinly populated peripheral areas (Richardson 1973). The theory has developed considerably through the years and has thereby taken on different forms. Weber was the very first to use the concept of agglomeration economies. He assumed that the advantages of clustering of economic activities lie mainly in lower transportation costs for mutual deliveries of input materials. The importance of these costs has decreased in the course of time; their share in the total cost of production is now far less than in the past. Along with the development of spatial theory, the scope of the principle of agglomeration economies has been widened considerably. It is no longer limited to material relations. This concept is also applied to immaterial relations such as exchange of know-how, service linkages and labour market relations. It is assumed that factors other than transportation costs cause the total production cost to be lower in urban cores than elsewhere. Those factors include information, communication and transaction costs. Furthermore, urban locations offer more than cost advantages. They provide a higher information density, a more differentiated and better qualified local labour pool, the presence of professional services, and greater possibilities for contracting out, which allows firms to specialize in their core activities. These opportunities all serve to make urban firms more advanced, more innovative, and therefore more profitable. In other words, agglomeration economies are not only 
expressed in lower production costs but also in so-called differential advantages. All in all, in the course of time, the interpretation of the concept of agglomeration advantages has been widened. From a concentration on transportation costs, the focus has shifted to the much wider concept of 'accessibility to factors of production'. This concerns the opportunity costs of time and money. As society becomes more and more complex, entrepreneurs must go to some trouble and expense in order to obtain information, to establish and maintain contacts, to enter into contracts, and to perform transactions in a given quantity and of a certain quality.

The invention and development of the idea of agglomeration economies has done no harm to the field of spatial economics. The concept not only helps to explain differences in economic prosperity between different regions, but it also has clear macro economic implications (Nijkamp and Davelaar 1989). The reason is that spatial economics provides some answers to the persistent questions regarding the origins of (technological) innovation and economic growth. This contribution seems to be penetrating into other branches of economics. In any case, the role of the local business environment is becoming a 'hot issue' in the debate about the competitiveness and thus the economic growth of the domestic economy. There is increasing awareness that the economic success of some nation-states rests on the achievements of certain areas within them (Pollard 1982). Also Porter (1990) emphasizes the quality of the production environment in explaining a country's economic success. He comes to the conclusion that "While economic geography has not been seen as a core discipline in economics, my research suggests that it should be" (ibid, 791, note 23).

To sum up, in regional science, theoretical work is dominated by the idea that levels of regional development can be traced to attributes of the business environment. These characteristics influence the behaviour as well as the prosperity of firms. This environmental paradigm underlies the explanation of the territorial equilibrium models of traditional location theory. At the same time, it is embedded in the explanation of the structural and cumulative inequality models of regional development theory.

This section offers some critical notes on the environmental paradigm in spatial studies. Its use is assessed regardless of whether it underlies spatial decentralization and interregional convergence on the one hand, or spatial concentration and divergence on the other. It must be emphasized, however, that the reduction of patterns of geographical development to characteristics of the territorial business environment is not devoid of internal logic. Actually, the ecological argument is rather self-evident and provides powerful explanations for general patterns of economic development across space. Nevertheless, several points of critique can be made on the presupposed influence of the environment. In particular, we dwell on how the ecological argument has found much support within the concept of agglomeration economies. Objections to its use can be raised on the methodological as well as on the theoretical plane. 


\section{Methodological problems}

Methodologically, the question is how to convincingly measure the role of the production environment in the behaviour and economic functioning of firms. Traditionally, spatial researchers have mainly used quantitative methods of statistical inference. These methods are, however, plagued by problems of internal validation. Do they adequately represent what they are intended to measure? Stated differently, an important problem of quantitative methods is how to interpret the statistical associations measured. Briefly, this section outlines the problem at hand. It uses three different clusters of approaches to measure the impact of elements of the production environment on the behaviour and performance of private firms.

Analysing aggregate business location The problem of internal validation lies primarily in the methods that relate the number of activities in a certain area to the environmental characteristics of that area. For instance, spatial agglomeration of firms in urban areas is almost automatically attributed to the impact of agglomeration economies. This should be recognized as dubious logic. Agglomeration theory predicts the clustering of activities but the concentration of activities does not prove the operation of agglomeration advantages. Britton (1974, 368-369) notes that the concentration phenomenon could be interpreted in behavioural terminology just as well or even better than in terms of true economic arguments. According to the behavioural approach, firms cluster together as a result of management's limited knowledge of less visible but equally suitable alternative locations. Their choice is often exercised by imitation in order to reduce uncertainty about competitive conditions, costs and demand.

Apart from imitation, there is another way to interprete clustered location patterns. This option is based on the results of studies of new firm formation. It appears that new entrepreneurs do not make active location decisions. They simply begin in their own living or working environment, irrespective of local economic conditions, (cf for example Gudgin 1978, and Cooper 1985). The growth of new firms will then eventually strengthen the existing spatial differences, for non-economic reasons,. This too can be denoted as a behavioural explanation for the location of businesses and firms.

If such behavioural standpoints hold true, the existing spatial pattern cannot be simply viewed as a causal necessity, at least not as an economic necessity. Nevertheless, this tendency is present in much spatial research. In this regard regional science deserves the criticism voiced by Braverman. From his point of view, the modern social sciences still suffer from the urge "to accept all that is real as necessary" and "all that exists as inevitable" (Braverman 1974).

Horvers and Wever (1989) express this discord between reality and necessity when comparing the locational behaviour of large internationals and migrant firms with the spatial performance patterns of successful existing firms. The former have a strong preference for the best-equipped locations with reference to the presence of universities, characteristics of the labour pool, the access to airports etc. That is not to say that these firms would function less well in other locations. According to Horvers and Wever, the decentralized location pattern of the second group, i.e. 'successful' or 'alert' existing firms (winner of awards, fast-growing small and medium-sized firms and businesses which use innovation stimulating measures), proves that less eye- 
catching, alternative, locations need not to hamper business performance. This possibly could be relevant and encouraging information for regional policymakers.

Analysing aggregate external linkages A second way to study the influence of the production environment is by analysing the spatial relations between organizations. Agglomeration advantages could, for example, be expressed in the locally oriented external linkages of firms in concentrated urban core areas. These linkages are abundant, as opposed to the sparse, long-distance linkages of firms located outside the agglomerations (e.g. Malecki 1991, Scot 1983, Czamanski 1976, Streit 1969). However, according to McDermott and Taylor (1982), even the mapping of patterns in linkages is not sufficient evidence for the operation of agglomeration economies. An unequal spatial dispersion of contacts can just as well result from the limited spatial information of firms. Local networks are then the result of a lack of knowledge on the part of the entrepreneur. He may not be aware of alternative actors who, though perhaps more appropriate, are located further away. McDermott and Taylor (1982) term this "a behavioural explanation of spatial interaction".

Analysing aggregate regional performance $A$ third method is the measurement of differences in performance between central and peripheral areas. This concerns indicators such as average regional income, value-added, regional production, investments, unemployment, employment etc. The problem is that these figures are usually only available as averages and aggregates. In those forms, the variation between individual activities is lost. Does the environment influence all firms to the same degree? Or does the aggregate conceal large differences between individual cases?

Even if the figures are available at the level of individual firms, they may still be ambiguous. It remains unclear whether spatial differences tell us something about the conditions of production in an area or about the businesses or entrepreneurs operating there. Some authors suggest that differences in regional development are not so much tied to variations in the production environment as to divergent production structures (for example sector and size differences). Saxenian (1981), for example, attributes the growth of Santa Clara County (including Silicon Valley) to the growth of the micro-electronics industry. However, other factors can generate regional inequalities. For instance, selective population shifts may result in "quality differences" in population structure (Keeble 1989, Wever 1986, Vaessen \& Wever 1986); regionally bound cultural factors (Illeris 1986, Brusco 1986, Bleumink et al. 1985), social psychological factors, and religious factors (McCLelland 1961), may also enhance inequality.

In sum, the argumentation about the significance of territorial conditions for economic growth is only valid when a causal link can be proven to exist between the characteristics of economic conditions of a business location and the profits, the total costs and/or productivity of individual firms. Obvously, gathering these data is no sinecure, so the researcher has to be satisfied with less. Gilmour (1974) suggests that these problems of finding appropriate empirical data for validating agglomeration theory result from its deductive nature.

We have pointed out the problems of measurement and interpretation. Yet, we have still not mentioned all the problems encountered when using the environmental 
approach. The ecological paradigm also has a number of theoretical deficiencies. These are illustrated in the next section.

\section{Theoretical problems}

But even if the influence of territorial conditions on economic growth can be measured in an unequivocal way, explaining economic advancement from an ecological point of view is still full of pitfalls. These problems arise from deficiencies at the theoretical level. In this section, we cover three points of criticism of a more theoretical nature regarding the environmental approach. First of all, there is the question of the origin of 'low-profile' and 'high-profile' production environments. In regional development theory, regional differences in production circumstances seem to appear out of thin air. McDermott and Taylor (1982) point out that some locational advantages - the presence of pools of skills, labour, services and infrastructure - tend to follow rather than precede the initial concentration of basic economic activity. This problem comes to the fore in an article by Scott and Angel (1987) about the development of the semiconductor industry in Silicon Valley. The researchers do not answer a keyquestion: Why did such strong agglomeration economies appear so early in Silicon Valley rather than at other nascent centres of integrated circuit production in the United States (in Phoenix and Dallas, for example)? Saxenian remarks that "The approaches of traditional location and growth theory thus fail to satisfactorily illuminate the processes of regional change which have occurred in Santa Clara County. Neither provides an adequate framework for understanding the electronics industry's original location and agglomeration and the resulting unprecedented growth" (Saxenian 1981, 4). The reason is that these theories conceive of regions as predefined spatial units, having dynamics in and of themselves and existing in isolation from the rest of the economy. In other words, regional dynamics is viewed as manna from heaven.

A second problem is the one-dimensional nature of regional development theory. It is only able to predict and deal with cumulative inequality between a given centre and an existing periphery. This makes the theory inflexible and vulnerable. Storper and Walker (1989), but also Saxenian (1981), point out that neither location theory nor regional development theory are able to explain the dramatic changes which have occurred in the arrangement of the space economy. These changes have been characterized by the creation and destruction of centres and peripheries. They have been a feature of civilization since ancient times. In these authors' view, the recent North-South and East-West shifts of economic activity are merely the most recent expressions of an age-old process. This leads them to conclude that "The evidence suggests that there is something terribly wrong with existing theories of spatial development, none of which provides a ready explanation for rapid growth at the periphery" (Storper \& Walker 1989, 18).

A third point of criticism is that the ecological approach - thus the principle of agglomeration economics as well - neglects the individual organization. According to McDermott and Taylor (1982, 7), "Industrial geography [and regional economics] has tended to treat organizations all in much the same manner - as black boxes, capable of a limited number of actions and responses, which are themselves embedded within, and their actions determined by particular environmental contexts." They further assert that "many studies in industrial geography remain external to the 
organization, taking the organization and its internal processes as given and having no direct bearing upon spatial patterns of production Nowhere is this stance more apparent than in the cost surface approach to locational explanation in which the organization is treated as a single plant firm which is no more than a locational 'actor' - one dot in a dot distribution" (ibid) Not only in location theory but also in regional development theory, there is no elbow-room for an action-taking entrepreneur or any independence of the individual enterprise from the environment, let alone any question of 'reciprocal influence' Critics use the term spatial determinism (Taylor 1986, Walker 1985) or spatial fetishism (McDermott and Taylor 1982) Walker (1985, 250)posits that "It [agglomeration theory] misses the possibility of action at distance and of active intervention of businesses in labour markets, local politics and the like Industry does not depend only on the passive action of the market, distance, and the cumulative weight of many industries to make places suitable for its purposes "

Such criticism is not recent but dates from the 1960s The fact that these points are still being raised is telling Apperently, the improvements on classical location theory within regional development theory have not resulted in a more active role for the entrepreneur and/or enterprise On the contrary, in the new off-shoot of regional development theory known as flexible agglomeration theory (Scott 1988a, Schoenberger 1987), the idea of agglomeration economies once again takes a predominant place.

However, with the neoclassical locational theories and the modern development theories, the arsenal of approaches in the spatial theorizing is not yet exhausted There are still other approaches, some of which directly contradict the premises of environmental determinism According to these approaches, the forces which control an organization's doings and fortunes are not to be found in the environment Rather they dwell inside the organization and its management

Inspired by the work of Simon (1957) and March and Simon (1958), the socalled behavioural school of economic theory arose in the second half of the 1960s The behaviourial approach tries to compensate for the lack of attention within classical economic thought for the internal aspects of the company The new approach focused on how economic choices come about and on the personal motives and behaviour of the entrepreneur In economic geography and regional economy, this means that attention is given to the underlying forces of locational behaviour as well as the spatial implications of behavioural patterns of companies This basic need to open up the 'black box of the firm' soon turned into a strongly developed network of subapproaches, with mutual points of contact but with just as many nuances of stress Seen from the company-environment perspective there are two tangled points within this dense undergrowth of behaviourist approaches which require some pruning These clusters consist of 'behavioural location theory' and 'geography of enterprise' 
Behavioural location theory crtiticizes a number of assumptions on which classical location theory is based. Classical theory assumes that entrepreneurs are omniscient and that they are exclusively geared toward the maximization of profits. Central to the behavioural critique is the notion that entrepreneurs do not have maximum knowledge and that "spatial organization of economic activities is, at any time, the product of both economic and noneconomic forces" (Pred 1966, 4). It is thus acknowledged that entrepreneurs act in situations of uncertainty with regard to the choice of location.

It was in the 1960s that Allen Pred made his appeal to embellish existing location theory by taking into account non-optimal behaviour, imperfect knowledge, other psychological variables, socially dictated constraints, and the impact of existing patterns on subsequent patterns (processes) (Pred 1967, 16). Pred does not in any way depart from classical location theory. Consistent with tradition, he concentrates on the single plant business. Furthermore, he incorporates Smith's (1966) neoclassical spatial margin concept into locational behaviour theory. According to Pred, some decisionmakers have plenty of (relevant) information at their disposal and the ability to use that information. Thus, he argues, they are in a position to make rational economic decisions. One comes across their enterprises at or close to the point of optimal of location. Of course, other entrepreneurs may have little information at their disposal and a limited ability to use it. It is likely that they will opt for a non-viable location. By the way, Pred does concede that companies can end up in the right place by chance. Between poorly and optimally informed entrepreneurs lie the majority, consisting of those who are non-optimally but adequately informed. They are not seeking the optimal but a satisfactory location (satisficing behaviour). We will not come across these companies at the point of optimal location (unless by chance). But we will certainly find them within the spatial margin where profit-making production is possible.

Indeed Pred's behaviouristic critique on classical location theory is very illuminating. Nevertheless, even in this train of thought, which is based on ideas of satisficing behaviour and nonoptimally informed decision makers, environmental factors get the upper hand over entrepreneurial action. After all, a lack of information creates uncertainty on the part of entrepreneurs. And uncertainty leads to a 'safe' choice of location. This means that business owners are inclined to imitate each other and that their locational behaviour is governed by a 'herd instinct' (Richardson 1973, 111). As a consequence, they have a preference for concentration areas, where many companies appear to be functioning well, i.e. they prefer the large cities. So, the same locational forces which are thought to optimize profitability also minimize uncertainty. The primacy of the environment remains intact and the environmental approach incorporates behavioural location theory with little difficulty. 
In the wake of the behavioural approach came 'the geography of enterprise' or 'corporate geography', a development of the 1960s. The geography of enterprise emerged out of the constant and ever-expanding criticism of (neo)classical location theory. The objections voiced by the behavioural location theorists paved the way for its arrival. Two points of critique triggered the emergence of this new field.

First, enterprise geography rejects the Marshallian idea of the single-plant family firm, which supposedly operates in a world of perfect competition and perfect information, as the predominant mode of industrial organization. The small firm could not remain the basis for modelling industrial geography forever. In the 1960 s it was recognized that the multidivisional, conglomerate and multinational corporations had swept across the United States, then Europe and finally the world. Their ascendancy had to be accounted for (Dicken 1986). McNee (1960, 202), the founding father of the geography of enterprise, noted that "The atomistic economy of yesterday, composed of millions of enterprises, is increasingly giving way to giant industrial combines".

The second point pertains to the predominance of classical economic thinking, with its emphasis on deterministic mono-causalities (Krumme 1969a). According to classical location theory, the location of economic activity is completely determined by environmental or locational factors. The actors are only capable of reacting to their environment by exhibiting mechanical relocation behaviour. However, through the spectacles of the corporate geographer, (large) firms are seen to have a measure of control over their environment. They can act on the basis of bounded rationality and make strategic decisions that enhance their corporate advantage.

Carr (1983) gives a rough outline of the development of enterprise geography. He suggests that it initially emphasized the adaptation of production and organization to conditions of the external economic environment. However, nearly all of the later behavioural corporate studies ignored the influence of the external economic environment on organizational behaviour. Attention turned towards the effects of organizational decision-making upon urban and regional development.

From this brief review two approaches can be distilled within the field of corporate geography. One focuses primarily on adjustment behaviour of (large) firms in response to environmental conditions. The other is oriented towards the regional impact of the structure and behaviour of large firms. The former mentioned approach incorporates the environment as an input condition to organizational behaviour. In contrast, the second treats the spatial and regional environment as an outcome of management decisions. We will refer to these two approaches in enterprise geography respectively as 'adaptation corporate geography' and 'regional development corporate geography'.

\section{Adaptation corporate geography}

The initial framework for adaptation was devised by Krumme (1969a,b) and Steed $(1971 a, b, c)$. In the field of corporate geography, the adaptation approach assumes that adaptive behaviour can lead to deviation from the optimum location (Carr 1983). Krumme even asserts that companies are rarely located optimally. This is because the 
optimal location is extremely unstable. Both in the external environment and inside the companies, the dynamics are constantly changing the operating requirements. And this makes the optimal location very changeable. However, the production conditions at a sub-optimal location are not necessarily detrimental to the individual enterprise. The management may be able to adapt the company optimally or nearoptimally without having to change sites. Decision-makers can even try to work their way into the environment and influence it to their own advantage.

According to Krumme, an entrepreneur is constantly trying to react to his environment in the right way. The 'optimum location' of the classical location theory is placed in opposition to the concept of 'the optimum path of locational adjustment'. Rather than circumstances in the external environment, it is the company's ability to adjust, including its locational behaviour (e.g. by relocation), which determines the prosperity of an area. Krumme (1969b) used three criteria to classify entrepreneurial action: the spatial dimension, the organizational setting and the time frame.

The spatial dimension The first criterion is the spatial dimension. Do companies react to changes in production requirements by making spatial or non-spatial adjustments? Spatial strategies change the geography of the enterprise as reflected in the number and the distribution of its facilities. Some common spatial adjustments are (integral) company relocation; setting up or closing down subsidiaries; and participation in, taking over of, or being taken over by companies that are better located. Non-spatial or in-situ adjustments occur at the present location. Some typical non-spatial adjustments are the more or less intensive use of existing means of production, introduction of changes in factor and/or production combinations, product or process alternations in response to environmental forces, and changes in linkage patterns.

The substitution principle gives entrepreneurs the freedom to choose between various means of adjustment. According to Krumme, to a certain extent - within the spatial margin - non-spatial adjustments constitute an alternative to spatial adjustments. Using Renner's (1947) terminology, Krumme (1969a, 35, note 18) contends that entrepreneurs are often able to substitute high 'economic efficiency' (the degree to which an entrepreneur's economic behaviour approximates the optimum) for low 'geonomic efficiency' (the degree to which an industrial location approximates the optimum). Figure 2.1 places this line of reasoning in a core-periphery context. It depicts graphically how adaptation geographers think organizational behaviour in smaller areas can substitute for the locational advantages of large urban regions. 


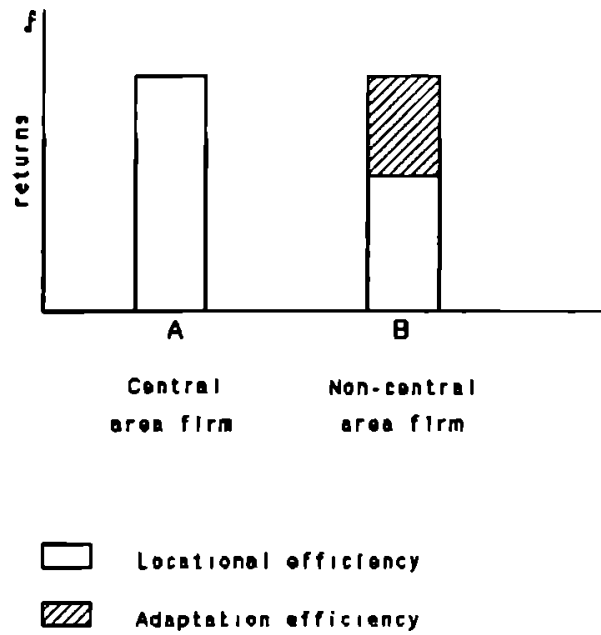

Figure 2.1 Substitution of low locational by extra economic or adaptation efficiency

Organizational aspects The second criterion for classification pertains to the organizational level at which a certain adaptation decision is made or at which it is to take effect. In this respect, a distinction has to be made between adjustment decisions at the corporate level, at the plant level, and at the level of the individual investor. Of course, the investor may or may not be the entrepreneur. The set of possibilities for spatial and non-spatial adaptation varies with each of these decision levels. Actually, this distinction reveals the great influence of both organizational structure and size on a firm's ability to adjust to and generate change. Organizational size in particular is considered to affect an organization's adaptability. More opportunities are likely to be available for larger firms. Large multi-locational enterprises have, as a result of their financial and managerial capacity, significantly more adjustment possibilities than small single-location enterprises. This applies to adjustments at the present location as well as to the possibility of constantly relocating assets across a number of sites (cf. also Rees, 1978; and Watts $(1978 ; 1980)$.

The time factor The third dimension is the time factor, i.e. the amount of time that is needed to carry out the necessary adjustments. Krumme makes a distinction between adjustments on a short, medium, and long term. In agreement with Steed (1971b), Carr (1983) adds that short-term adaptations consist of immediate reactions on the part of entrepreneurs to the external environment. The entrepreneur responds without lengthy discussions or radical investment decisions. Adjustments over the longer term, on the other hand, are more geared toward gathering as much information as possible and influencing the modifications in the external environment to one's own 
advantage Moreover, as uncertainty in the environment increases and the rate of change rises, the short-term adjustments will become less effective

Krumme (1969b) reasons that these criteria - space, organization, and time are interrelated In the short term, in-situ changes are attainable, whilst in the long term spahal adjustment strategies are possible In all instances, multi-locational companies have more adjustment options than single-location companies Krumme classifies the possibilities of environment adaptation according to the following three scenano's (see also Carr 1983, 393-394).

1) The single plant can adapt in the short run The intensity of use of investment goods can be vaned and input factor combinations can be changed as long as new investment is not necessary In this way multi-plant corporations can make spatial adjustments at different locations to various degrees

2) New or abandoned investment, is a feature of medium-run adaptation, when it does not involve the addition or removal of totally independent production units Again, the scope of adaptation is less for the single-plant firm Mediumrun adaptation can include agreements and cooperation between firms This may entall production integration, subcontracting, and research and development collaboration

3) Long-run adaptation involves locational investment strategies These include disinvestment, relocation, establishing branch plants, or change in ownership (by way of merger or takeover) The scope of adaptation is wider for the larger-scale, multı-plant firm that is planning over the long term

In sum, adaptation corporate geography focuses both on the internal milieu of companies and their external interaction The environment, as it expresses itself in the external economic conditions, has not been given much attention until recently (Dicken 1990) The bulk of corporate geographical studies of the mid-1970s and 1980s focus on business strategies These studies trace the effects of those strategies upon spatial divisions of labour and regional development

\section{Regional development corporate geography}

From the end of the 1970 s onward, the geography of enterpnse became strongly biased towards the spatial ramifications of decision-making within large firms (Carr 1983) Spatial analysts were impressed by the powers of the modern corporations and their area-organizing capabilities, as compared with the small firm The locational strategies of large firms and the way they organize their internal activities profoundly shape urban and regional economies Thus, it has been argued, large firms tend to create regional inequality (Watts 1980b, Dicken 1986) Because of their financial, economic and political power, it is assumed that large corporations possess considerable autonomy in making geographic decisions Place relationships are organized and regulated according to elaborate, pre-formulated, corporate plans for survival and growth Corporate autonomy makes the objectives set by the management extremely important The traditional theory of the geography of price has become fused with or even swept away by the geography of administration

Recently, Walker (1989) made some order out of the amalgam of corporate studies The literature on corporate geography seems to revolve around four main issues (1) the spatial bias in location introduced by corporate calculation, (2) the 
corporate spatial division of labour on regional development, and (4) the geographic expansion of corporate activities

The first issue, mainly concerns the reasons why large firms, unlike small ones, can manifest spatial patterns deflected away from the optimal or least-cost location Locational and organizational adjustment behaviour forms the empirical basis of these studies They therefore adhere to an adaptive approach, which has been addressed in the previous subsection The second to fourth issues, however, are more amenable to what we will term here a corporate regional development approach They make up the bulk of studies in the field of the geography of enterprise and will be discussed bnefly below

Corporate spatial divisions of labour Some corporate geographers who view uneven development as the outcome of management decisions and organizational structures of large corporations, link up with the new international division of labour theory of Hymer (1972, 1976) and Froebel, Heinrichs and Kreye (1977) In this view corporations are endowed with an internal division of labour, internalized systems of commodity and service flow, multilocational operations, and a herarchical mode of organization The argument centres on the general distribution of such corporate facilities as headquarters, research laboratones and manufacturing plants This division of labour within the corporation, it is said, is marked by a vertical hierarchy which becomes imprinted on the national and global space-economy, as first proposed by Hymer Walker $(1989,48)$ outlines the general spatial pattern according to this approach "the headquarters of giant multinationals are concentrated in core cities of developed countnes, higher order functions are grouped in secondary cities, and lower order activities are shunted off to backward regions or third world countnes"

Corporate branch plants and regional development Another strand of research in the corporate regional development approach concerns branch plants Many branch plants are in peripheral assisted areas They are depicted as detrimental to regional growth for two reasons First, branch plants have relatively few local linkages, hence low income multipliers (Townroe 1975, Britton 1976, Britton and Gilmore 1978, Watts 1981) "They are 'cathedrals in the desert' by virtue of their low impact on their immediate surroundıngs" (Walker 1989, 48) Secondly, branch plants are unstable contributors to regional prosperity, prone to closure (Erickson 1980, Bluestone and Harrison 1982)

Corporate spatial expansion Other investigations in the geography of enterprise are concerned with the geography of corporate growth It has to be admitted that these studies have but indirect links with regional development issues In such corporate growth models, the large firm creates a distinct pattern of spatial expansion It establishes a core territory within which it conquers its immediate competitors that territory forms a base from which the enterprise gropes outward in search of new markets and resources Some well-known spatial models of corporate growth are those of Taylor (1975), Watts (1980a) and Håkanson (1979) Watt's model is concerned with external growth (1 e merger and acquisition) Haikanson's model deals with internal expansion of firms (establishment of sales offices and production plants) 
The domunant stream of research in the geography of enterprise focuses on big enterprise strategies and structures, tracing their effects upon communities and regions (issue two to four) Thus the emphasis has shifted away from the issues preeminent in traditional location theory and regional growth theory These theories are concerned with the influence of the spatial dimension in the economic environment (1 e proximity to raw materials, markets etc) upon industrial location and performance Corporate geography seems to be avoiding a theoretical confrontation with (neo)classical location theory and modern regional development theory

Behaviourial geography, particularly in the form of the geography of enterprise, has clearly enhanced spatial theory's sense of reality Nevertheless, there is a long list of objections to this approach The general criticism is that enterprise geography suffers from an inadequately developed theoretical framework and an uncontrolled growth in the applied methodology (Dicken 1990) Partly because of this, both the value of its predictions and its practical application in policy-makıng are limited (Carr 1983, Hayter and Watts 1983, Walker 1989) Further points of criticism are specified below In order to meet the objective of this research - explaining small business growth in contrasting environments - these objections to the corporate geography approach have to be dealt with Stated briefly, the shortcomings dealt with are the neglect of the small firm, treating enterprises in isolation, and, if any, an inadequate focus on an organization's environment from a geographical point of view

\section{The neglect of the small firm}

The geography of enterpnse neglects small and middle-sized companies Whilst interest in the small company has grown since the 1970s, the geography of enterpnse is still strongly fixed on the large company, which it views as a 'given fact' How large companies get started and where they come from are questions the geography of enterprise does not ask It ignores the role that space and place might play therein Apparently, its practitioners are unaware that large companies must once have been small This fixation on the large company diverts attention from the central problem in regional development theory, namely the origin and growth of firms Instead, attention is directed toward the distribution of economic surplus '[Geography of enterprise] treated the problem of production as basically solved, in order to focus on distributional matters " (Walker 1988, 379)

\section{Firms in isolation}

The enterprise approach is too much of a single-actor or single-region approach It does not compare the functioning of companies in different types of production environment with each other However much behaviourial theorists emphasize the adjustment possibilities of entrepreneurs, in order to avoid a spatially deterministic explanation they still have to explain a paradoxical situation How it is that Company B, which is located in a badly provided-for (i e peripheral) environment, can make adjustments and overcome its locational disadvantage relative to Competitor $A$, which 
is located in a well provided-for (central) environment? Krumme's assertion that managerial adjustment behaviour can compensate for an inadequate location (Figure 2.1) is rather tricky as it ignores a firm's competitive context. His substitution framework passes over the following problems. In the first place, even the most staunch adherents of the behaviourial approach cannot deny that adjustment strategies may be combined with extra investments. This combination, in tum, pushes up production costs (see Townroe 1974). We should recall that the costs of site relocation are second only to bankruptcy as a dramatic event in a company's life (cf. Gruijthuisen and Junge 1992). But also other adaptation measures involve considerable expense, such as setting up internal supply capacity whenever the environment is inadequate in that respect, or training personnel if the regional work force does not come up to scratch. Behavioural studies have the tendency to neglect the costs which result from the decisions and actions of entrepreneurs. These are depicted in Figure 2.2 as adaptation costs. If these costs are taken into account, then we must consider whether adjustment measures can indeed be efficient and viable.

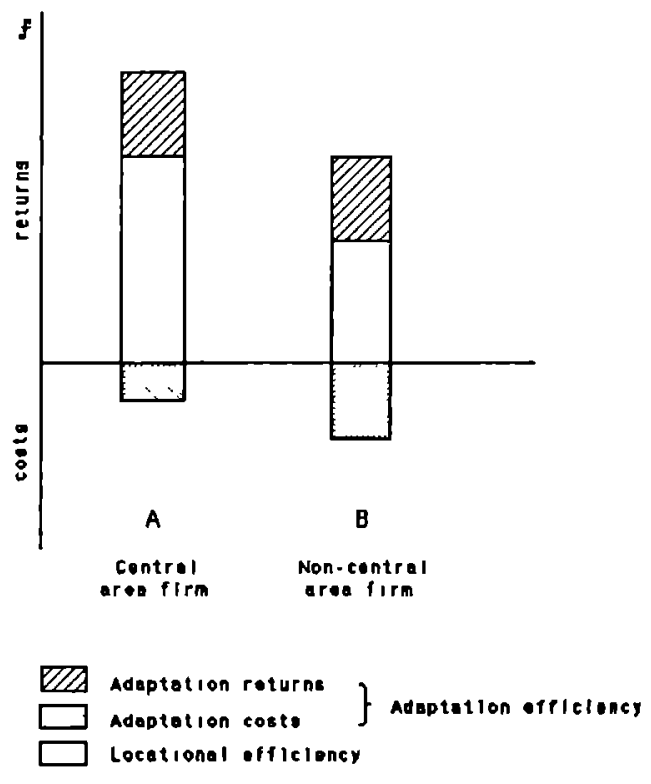

Figure 2.2 The fallacy of the substitution framework

Do the benefits exceed the costs? Small high-tech companies in peripheral locations might have to dig deeply into the coffers to acquire highly trained staff. Whether these recruits are found by in-house training or by attracting them from far and wide, the investment may be out of proportion to the returns. But even if this is the case, we are still not home and dry. There is a second possibility. In principle, every 
catching-up manoeuvre of Company B depicted in Figures 2.1 and 2.2 can be responded to by Company $A$. The latter maintains the initial difference in production costs and, in so doing, sustains its initial competitive advantage. If $B$ puts its staff under pressure to produce more efficiently, then $A$ can do likewise. If $B$ tries to raise productivity by introducing new techniques, then $A$ can do the same. If $B$ brings a new product onto the market, then A can reply with a comparable product. In other words, A does not even need to be as good an entrepreneur as B. All that A needs to do is to keep a close eye on B and respond to each of B's strategic moves. In other words, $\mathbf{A}$ just has to be a good imitator. It is also possible that A's adjustment costs will be lower than those of $B$ as a consequence of agglomeration economies (Figure 2.2). Thus, the more $B$ fights against his location, the more he can drop behind $A$.

This scenario demonstrates the theoretical consequences of the claim that enterprise prevails above production environment, or that disadvantages of the environment can be compensated by extra initiatives on the part of the entrepreneur. If we nevertheless reject the spatial deterministic undertones in regional economic analyses, we have to find solutions for the above-mentioned problems. Krumme and Renner fail to do this in their spatial interpretation of the substitution principle. Chapter Six attempts to meet these shortcomings by taking a closer look at the efficiency of organizational adaptation in a core- periphery context. There, we intend to throw light on the underlying reasons why locational disadvantages need not necessarily get a grip on sub-optimally located firms (irrespective of industry or market structure). The main argument evolves around absolute or differential advantages which can accrue to a firm from responding to environmental constraints. These advantages can downplay the role of the production costs argument.

\section{Inadequate conception of the relevant environment}

The third point of criticism is that the geography of enterprise either fails to define the environmental context or defines it inadequately for explaining regional development patterns. In so far as the environmental context is taken into account in the geography of enterprise literature, (empirical) research is one-sided. It is focused on the spatial consequences of the firms' strategic reactions to economic and technological change in the global environment (see De Smidt and Wever 1990b; Kelly and Keeble 1990; Fuchs and Schamp 1990; Le Heron 1990; De Smidt 1990; Chapman and Walker 1987, 122). Put differently, corporate geographical research perceives the environment mainly as an output of organizational action. In sofar as it views the environment as an input condition it considers only the influence of general socioeconomic trends, such as technological developments and changes in consumer demand. Meanwhile, it neglects the impact of unequal production conditions across space on business operation. Dicken warns against this ommision in corporate geography. "Certainly, we should be just as concerned with the influence of geographical variety (economic, political, social, and cultural) on corporate behaviour and strategy as with the influence of corporate behaviour and strategy on geographical space and geographical place" (Dicken 1990, 241).

The neglect of corporate geographers to consider the geographical environment as an input condition to organizational action and performance undermines its own justification. As a result of this ommission corporate geography has failed to develope its own stance on the subject of the role of comparative advantages and 
agglomeration economies on organizational action and operation. This vacuum seems to be surreptitiously filled in the geography of enterprise literature with principles borrowed from environmental approaches in regional sciences (i.e. location theory and regional development theory), as it hardly can be a coincidence that corporate geography detects exactly the same spatial hierarchical structures and patterns of regional inequality as regional development theory does (see pages 19-20). The difference is that the geography of enterprise claims that these structures are the results of spatial corporate strategies and exploration. However, the work has not been done to sort out the organizational impact on the spatial structure of the economy from other forces such as (Weberian) cost conditions and other locational attributes. Therefore, we agree with Walker that the case for a distinctive 'corporate geography' in the modern world has not been made yet $(1989,50)$. Walker illustrates this point clearly. He notes that the hierarchical spatial structure of large corporations follows spatial differences in economic conditions and does not precede them. For example, corporate headquarters and R\&D laboratories are located in major city centres to gain access to 'business services' and 'high quality personnel' already concentrated there, not vice versa. Similarly, cheap labour conditions in the periphery precede the location of corporate production plants, and not the reverse. "There has been, in the end, a notable failure of 'geography of enterprise' to show how large firms' spatial evolution is different from geographical industrialization under capitalism in general" (Walker 1989, 50).

In the above, we first dealt with viewpoints in spatial analysis which beatify the role of the environment. We subsequently dealt with approaches explaining the spatial structure of the economy in terms of organizational behaviour. Then we established that unidimensional reasoning from either of these points of view leads to unacceptable and incredible premises from the other perspective. Now the question arises whether the two standpoints can be linked or integrated. Dicken (1990) makes an emphatic plea for cross-fertilization between contextual and organizational approaches. Up to now this has hardly occurred.

\subsection{Linking environmental and organizational approaches}

Hayter and Watts (1983) point out that most analyses of the interdependence of companies and environment apply a case-study approach. In so doing, these analyses underline the difficulty of incorporating the relationship between companies and environment into models of regional economic analysis. Nevertheless, models of spatial analysis almost never deal exclusively with either the environment or the entrepreneur. Actually, most models have the potential for blending an environmental with an enterprise approach. Thus, theories of regional development, in which agglomeration economies play a vital role, incorporate elements of behaviourial approaches as well. Conversely, corporate geographers, now more than in the past, pay attention to the environmental context in which enterprises operate. In this section we will discuss alternative ways to link these approaches. 
Growth pole theory

Some spatial extensions of Perroux's growth pole concept (Boudeville 1976, 1977) pose that when one or more big enterprises in a propulsive industry are established in a depressed region, development in this area can be turned around This supposition implies an alliance between an organizational and an environmental approach The organizational aspect is present in the assumption that the dominant, growth-inducing firms are able to survive and transform the adverse environmental conditions (i $e$ stagnating regions) The environmental aspect is linked to the growth pole theory mainly by way of the above-mentioned propulsive firm The arrival of such a propulsive firm or unté motrice improves external production conditions for new or existing local firms, which can take advantage of the extensive input and output requirements of the propulsive firm ${ }^{1}$ It must be noted, however, that writers are ambiguous about the locational requirements of economic growth-inducing activities Early geographical interpretations of Perroux's aspatial growth pole theory assumed that big powerful enterprises would be able to trigger economic development in retarded areas This implied a certain organizational capacity to overcome adverse local conditions Later, when the shortcomings of this approach as an instrument of regional policy became apparent, critics emphasized that growth poles cannot be established everywhere They were only feasible in large urban centres, already endowed with sophisticated production conditions (HIiggins 1988) With that, the organizational aspect was shoved to the background, and environmental determinism took the upper hand Another drawback of the theory is that it does not explain growth Instead, it takes growth as a point of departure, stating only that it stimulates other economic activities (Jansen 1970)

\section{Product life cycle theory}

A second model in which firm size plays a role in linking an environmental or ecological approach with a behavioural or organizational approach can be inferred from the product life cycle theory (Vernon 1966) According to that theory, a product goes through several stages of development during its 'life' In the innovation and early growth stages, the firm is small and dependent on skılled labour, such as scientists and engineers, for product and process innovation Therefore, producers seek out high-quality locations in the established cores any of the advanced countries of the world There, communication is swift and easy, and external economies can be tapped In subsequent stages of product development, when the product reaches maturity, geographical market extensions and declining production costs come to determine sales and profitability of the product At these stages, manufacturers begin to shift production to distant and less-developed, low-cost regions and countries

It cannot be demied that environmental variables, such as market opportunities and lower labour costs, are assumed to be the prime impulses for manufacturers to internationalize production Nonetheless, Krumme points out some behavioural or adaptive elements which invade Vernon's model as products mature Krumme notes that "One of Vernon's specific concerns is with a corporation's 'public' relationships to its host country A multitude of barners resulting from the corporation's foreignness 
have to be met by varying degrees of personal, organizational, and technological adaptability to local conditions in order to prevent these barriers from influencing the corporation's development" (Krumme 1969a, 39). Furthermore, he adds, "One does not need to go all the way to Bavaria or Hawaii to realize that such barriers do in fact exist also on an interregional level within countries".

Summarizing, in the innovation and early growth stages, when the firm is still young and small, its growth fully depends on the quality of the external environment. However, this changes when the product reaches stages of maturity and the firm has grown into a powerful, vertically integrated, multi-plant company, located in peripheral regions and nations. Then it encounters a multitude of barriers imposed by the spatial environment. By that time, the survival of the business depends on its capacity to counteract environmental constraints.

\subsubsection{The market structure link}

Scott (1983) and Storper and Walker (1989) present a very different model of geographical industrialization. They observe that new industries tend to emerge in new places, the so-called growth peripheries. They argue that new or revived industrial sectors often require totally new factors of production, not pre-existing. So, at its inception, a new industry has to create its own inputs. It is not enough to simply compete for a stable quantity of goods and labourpower. In the early phases of development (or renewal) specific material parts and equipment may be so novel that they have to be produced on the spot. Similarly, the labour skills are likely to be so new that they can be acquired only through practical experience and on-thejobtraining. The huge production costs which accompany these investments can be covered because of the limited competition and huge profits in the early growth stages. It follows that new or leading firms in new industrial sectors have a substantial amount of freedom to develop right wherever they are or to locate where they please. In its early stages, a new industry can settle on any one of a wide variety of locations. These moments of enhanced locational freedom may be seen as windows of locational opportunity (Scott and Storper 1987). So new industries may spring up in unexpected places. Actually, industrial history is replete with such cases. Storper and Walker (1989) mention many instances, of which we can mention a few here. "Henry Ford founded his firm in Detroit, a minor city, and made it the center of the US (and world) automobile production in the 1910 s by virtue of his Model $T$ design and assembly line methods... John Deere, a blacksmith from Grand Detour, Illinois, went no further than Moline to build better plows, and northern Illinois became the US (and world) focus of agricultural equipment production... Santa Clara County, Phoenix and Dallas, all relatively obscure sites, became early homes of the new semiconductor industry in the 1950s and have prospered mightily since... In Japan and Korea, supertankers and video recorders are examples of new industries appearing altogether outside the dominant industrial complexes of the Atlantic economy after World War II" (ibid., 71-72).

However, locational freedom does not last forever. As the industry matures, price competition between firms intensifies. Total costs of production increasingly 
determine profit rates, and spatial cost differentials begin to play a part in the competitive struggle. Then, locational windows of opportunity diminish drastically. One start-up area, that with the most favourable economic conditions, surges ahead, while others decline or grow more slowly (ibid., Figure 3.1, 71). In other words, the environment has gained primacy over entrepreneurial action.

The model of geographical industrialization developed by Scott, Storper and Walker suggests that spatial diversity of location patterns within an industry is a short-term phenomenon in the business cycle. Here, we take a different view. We posit that locational diversity is not merely a cyclical phenomenon but contains a structural component as well. Competitive firms located outside existing core territories can be found at any time, albeit in some periods more intensively than others. The point is that the model of Scott and Storper and Walker is not able to explain this structural component in the diversity of location patterns.

\subsubsection{The environmental dimension link}

In this 'method', the either implicit or explicit difference between the non-spatial and the spatial dimension of the environment is functional. This distinction infiltrates the 'new' theory of flexible production complexes.

\section{Flexible production complexes}

Scott's theory of the geography of flexible specialization (Scott 1983; 1988a,b) is a spatial interpretation of Williamson's (1975) transactional cost approach. Scott's point of departure is the way the industrial sector adapts to a number of socio-economic trends, particularly the crisis of mass-production. This crisis is associated with "intensified uncertainty and instability of production, and increased competitiveness in final markets" (Scott 1988b, 176). As a consequence, firm structures and strategies in the advanced capitalist countries are thought to change dramatically. Mass markets have become saturated and unstable. Competitive firms have been responding to these shifts by "experimenting with flexible forms of organization which permit rapid shifts in output" (Sabel 1989, 18). The central issue in organizational management is how to create a flexible management and production system that can react to new technical and market opportunities. In order to achieve flexibility in the deployment of labour and capital, enterprises increasingly contract out activities formerly undertaken within the firm. Meanwhile, they foster interfirm relations and transactions. Inter-establishment interactions and transactions are becoming more important. At the same time, the costs of "transport, communication, information exchange, search, scanning, and so forth" (Scott 1988a, 161) become more significant. These transaction costs are sensitive to distance: "the greater the spatial dispersion of producers, the more onerous these costs will be" (ibid., 176). By lumping together, companies can minimize these potentially onerous transaction costs and continue producing economically. On the other hand, where enterprises are located in close proximity, they develop a complex and dynamic network of relationships. In stark contrast to this are the areas with a low level of economic activity, i.e. peripheral areas. In similar regions, the transaction costs can rise too high for the contracting out of supportive tasks to 
remain viable. Companies in these areas tend to internalize activities and display a limited degree of external integration. Consequently, in a changed economic environment, the production of goods and services in non-central areas is now less efficient than in the centre. Scott identifies a renewed tendency toward the spatial concentration of economic activity (Scott 1988a,b). This concentration occurs not only by means of active location decisions on the part of enterprises but also as a consequence of spatial selection processes.

We can infer that, according to the flexible agglomeration approach, the successful adaptation to macro-economic and technological changes is dependent upon the organization's spatial context. Put differently, companies are assumed to be basically able to adapt and respond to economic and technological forces effectively, but not to territorial hindrances. Thus, when all is said and done, spatial determinism comes off remarkably well in this approach.

\subsubsection{The national development link}

We mentioned above that economic developments are spatially dispersed and highly differentiated in the Netherlands. The 'urban field theory' of Friedmann and Miller (1965) offers a popular explanation for these features of the Dutch space economy. On the basis of developments in the United States, Friedmann and Miller discuss the ever-widening patterns of interaction in the highly developed world. As a consequence of this process, urban living will extend far beyond existing metropolitan cores and penetrate deeply into the periphery. The older established centers, together with the incorporated enveloping periphery, "will constitute a new ecological unit of America's post-industrial society that will replace traditional concepts of the city and the metropolis. This basic element of the emerging spatial order we shall call the urban field" (ibid., 313). Increasing real income, increasing leisure and increasing mobility make urban populations spread out over surrounding peripheral areas, attracted by their space and scenery. Thereby, the full effects of the combined trends in income and leisure will be transmitted through the increased mobility which modern technology affords. In their 1965 article, Friedmann and Miller focus on trends in the spread of population. Yet like many others, they also apply this line of thought to the location of economic activities. It is assumed that improvements in transportation and communication methods progressively lessen the friction of distance. These developments thus diminish the force of spatial proximity, expand the spatial outreach of agglomeration economies, and consequently allow industries to disperse their facilities. At its most extreme, this line of thought is expressed in the belief that industry is no longer bound to particular places at all. Supposedly, it has become 'footloose', free to follow the whims or residential preferences of owners and managers (Berry 1972). In the Dutch context, this would mean that the favourable conditions of the metropolitan core region in the western part of the country, i.e. the Randstad, have become accessible and available to firms located at a considerable distance. It is assumed that almost the whole country lies within the 'urban field' of the agglomeration economies of the Randstad. Lambooy (1976) and Lambooy and Huizinga (1977) already recorded these processes in the mid-1970s. 
However, there is much debate about the extent to which a lessening of the friction of distance already has or is currently taking place. Processes such as increased need for information, communication, and face-to-face interaction run counter to the widening patterns of interaction. Thus, these processes impede the advancement of those patterns. Furthermore, enhanced mobility is turning against itself. Growing congestion, manifest in endless traffic jams, train delays, accidents and many other inconveniences, curbs the growth of mobility and consequently endangers the access of outlying area firms to resources and support in central regions.

Apart from increased mobility, there is another factor associated with economic development which causes wide dispersal of economic activities across space. Decentralization also occurs because of improvement in the quality of the regional production environment on the spot. In highly advanced post-industrial communities like the Netherlands, prosperity and infrastructural services are spread across the country in such a way that economic conditions are favourable for manufacturing virtually everywhere. The bigger the pie of national prosperity, the larger the piece for creating a favourable production environment in lagging regions. Moreover, it is no surprise that national prosperity leaks through to outlying areas more intensively in small countries than in vast nation-states (Friedman 1966, 12). In the Netherlands, social, educational, cultural, infrastructural and other services are maintained at a high level, even in the most outlying areas. That is not to say that the external conditions for economic production are evenly dispersed in the Netherlands: they are not. If anything, this gap between large urban core areas and smaller outlying regions will widen as the process of technological development continues. Major technological improvements will be either absorbed by or directed to the major cities earlier, and on a larger scale, than to the small towns. In the same way, the telegraph and the railroad increased the relative advantages of big cities over small towns and rural settlements in the nineteenth century (Pred 1974; 1980). Nowadays, exactly the same thing is happening with respect to telecommunications and high-speed rail transport, for example. The large cities are receiving more such amenities than small towns and rural areas.

There is no reason to believe that differences in economic conditions between the core and the periphery have been evened out in a relative sense. That does not alter the fact that circumstances in the periphery have reached an acceptable absolute level, which no longer hampers the growth and survival of firms. The remaining locational disadvantages in the periphery can be handled by the entrepreneurs themselves by means of adaptation behaviour (Horvers and Wever 1989). It is assumed that the level of economic accumulation in a company's environment affects the development of the industrial sector up to a certain point; beyond that point, its influence is much less. This threshold has been exceeded throughout the Netherlands, with the possible exception of the two most northerly provinces. That means that geographical differences in the functioning of the industrial sector cannot, or at best only in small measure, be traced to attributes of the regional production environment. Other factors are therefore more relevant. These include the element of chance, the quality of the region's management, the production structure, historical and cultural factors, and others (Wever 1985, 1991; Horvers and Wever 1989).

Thus urban field theory implies that the balance between organization and environment in explaining business growth and performance across space is influ- 
enced by the grade of macro-economic advancement. The more a country is technologically developed and economically prosperous, the less importance may be attached to environmental space as a hard regional differentiating factor. This is because in the developed world the accessibility and spread of economic facilities is so wide that even deeply in peripheral areas no prohibitive barriers for business growth need occur. Remaining locational disadvantages for outlying area firms vis-àvis competing firms in urban core areas, are thus for the former to tackle for themselves $^{2}$. Similar to the adaptation framework, as depicted in Figure 2.1 (page 28), urban field theory leaves more room for the entrepreneur to manoeuvre against his area of location than other hypotheses of regional development. The challenge is to fill in this space. Therefore, the environmental responsiveness of small firms has to be made actually visible and the problems attached to this line of thinking (section 2.3.3) have to be solved.

\section{$2.5 \quad$ Conclusion}

Spatial theories concerning the firm-environment debate can be divided into two broad approaches. One assigns primacy to the environment over the firm. The other, in contrast, assigns primacy to the firm over the environment. The former includes traditional location theories as well as modern regional development theories. It has come to dominate theory-building in regional science. Organizational approaches, such as locational behaviour theory and corporate geography, have never been able to develop an adequate theoretical framework as an alternative to the ecological approaches. Walker (1989) even writes a requiem for corporate geography. However, by abandoning alternative frameworks, one does not absolve the criticism of the prevailing environmental paradigm from which those alternatives emerged. One such point of criticism concerns the subservience of the individual entrepreneur to the dictates of the surrounding economic environment (Chapman and Walker 1991, 25). In both location theory and regional development theory, the individual entrepreneur is deprived of any ability to counteract his regional context. Furthermore, empirical research is crawling with contradictory observations and exceptions to the rule. The prevailing ecological frameworks in regional science are not able to deal with these counterfactuals adequately. On the other hand, some attempts to deal with growth in the periphery have recently been made (Storper and Walker 1989). Thus, theories of regional development, in which agglomeration economies play a vital role, are now incorporating elements of organizational approaches as well.

We have evaluated several opportunities for cross-fertilization between ecological and organizational approaches in regional science. This exercise reveals that the urban field approach, in an extended and moderated form, provides a suitable theoretical starting point for dealing with empirical diversity in the location of economic activities. A moderated urban field theory downplays the comparative advantages for economic activities in the urban core regions of the advanced economies of the Western hemisphere. But it does not assume them to be completely absent for every kind of activity, at any stage in its life cycle and with respect to all factors of production. The point is that due to national prosperity economic conditions 
in outlying areas have become improved such as to meet minimum requirements for succesful production. The remaining locational disadvantages in the periphery are assumed to be handled by the firms themselves, by means of adaptation strategies. It is this bounded organizational adaptive capacity which is supposed to explain small business growth in relatively unfavourable environments. This thesis intends to carry further an adaptation perspective in the field of regional science. In order to buttress such an approach in regional science, the next chapter lays its ears to the ground of another discipline in the field of economics which is as preoccupied with firmenvironment relationships as is regional science.

\section{Notes}

1 In addition to this the environmental aspect plays a role in growth pole theory in the debate about which environmental conditions must be fulfilled to make motor industries locate themselves in retarded regions.

2 On the other hand, an ecological approach, which explains regional development patterns and differences predominanly from the quality of the regional production environment, is preferable where the level of development is lower, i.e. in developing countries. In these countries accessibility and spread of elementary economic facilities transport and telecommunication infrastructure, skilled labour, supplies, services etc. is so constrained that business growth is virtually impossible in non-central locations. 


\section{Chapter Three}

\section{Organization and environment: the perspective of organ- ization theory}

\subsection{Introduction}

The previous chapter exposed the need for improving the theoretical foundation of organizational autonomy and adaptation in spatial science. It also made a case for extending that theory to embrace small and medium-sized enterprises. The present chapter looks for clues in the administrative literature to counter the inadequacies of spatial theory. Can the field of administrative science indicate how to create more leeway in regional economics for organizational sovereignty, particularly for small and medium-sized enterprises? To answer this question we should conceive of central business locations as favourable and peripheral ones as adverse environmental operating conditions. How does the business administration literature in general, and organization theory in particular, deal with the quality of the external production environment? Does organization theory elaborate on the balance between organizational behaviour and environmental conditions as an explanation of economic performance? At first glance, there is some indication that organization theory might provide some insight. In regional research, the enterprise approach has never posed a serious challenge to the environmental or ecological perspective, while in organization theory it has. For a long time, the organizational adaptation view actually dominated this field of research (Pfeffer 1982, 41; Hannan and Freeman 1977, 291; Astley and Van de Ven 1983, 253).

However, to assess whether regional science can really learn anything from organization theory about the interaction between firm and environment, we first have to identify which environmental concepts both approaches are using. Are the concepts of the administrative and spatial environment related to each other? How can these concepts be integrated? After this assessment a second question comes to the fore. What perspectives does the administrative literature take in regard to organizational action and the role of the environment? How do these perspectives compare to the views prevailing in regional science? The third question concerns the role of size. In brief, the problem is that adaptability to environmental conditions is a function of organizational size, while the ability to attain substantial size depends on an organization's capacity to adapt. 
What struck me while browsing' through the organization literature is the increasing attention devoted to organizations' environments in the course of time. In this respect, organization theory has come a long way since McDermott and Taylor (1982, 77-78) noted that this discipline conceptualized the environment, albeit in an essentially abstract manner. In the past two decades, several organization theorists have tried to structure this reservoir of 'everything outside the organization'. They stratified the environment according to levels of specificity, immediacy, and relevance to the organizational phenomenon concerned (cf. Beard and Dess 1988; Castrogiovanni 1991; Starbuck 1976). Moreover, for the sake of comparison, theorists have described the basic character of environmental elements in terms of various theoretical dimensions (cf. Aldrich 1979; Child 1972; Dess and Beard 1984; Mintzberg 1985). Thus, it is argued that environments are both multilevel and multidimensional (Betton and Dess 1985).

\subsubsection{Environmental levels}

The well-known structural contingency model (Petit 1967) conceptualizes the environment as a stratified structure. This model hypothesizes that organizational structure and performance are largely dependent on environmental change and complexity (Clark 1985). The model, which has also infiltrated industrial geography, was analysed by McDermott and Taylor (1982), who distinguish four environmental layers (Figure 3.1).

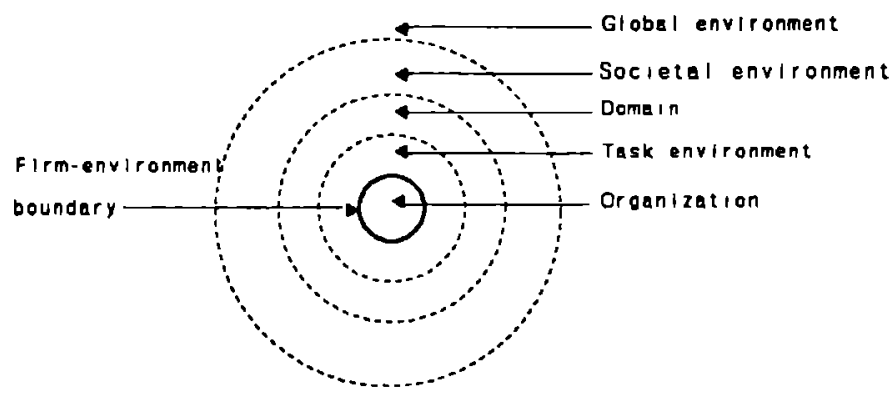

Figure 3.1 Levels in the environment according to McDermott and Taylor (1982, p.78)

These layers are identification points along a continuum depicting the directness or indirectness with which environmental forces impinge upon the operations of a focal organization. The organization is conceived as being confronted at the outer level with a global environment, which represents all knowledge, technologies and linkages. 
The societal environment can be regarded as the relevant cultural, political, social and economic system context, within which technologies are embedded and evolve. Within the societal environment, a particular focal organization establishes an environmental domain. This comprises the set of organizations with which a focal organization might potentially interact, given its particular character, the inputs it requires, and the output it produces. It is delineated by the industries or sectors with which an organization might interact (McDermott and Taylor 1982, 19). In passing, McDermott and Taylor's domain environment is similar to Castrogiovanni's (1991) aggregation environment.

Actually, the organization or enterprise will contact only a portion of the domain elements. This particular set of trading, business, labour and information contacts is called the task environment or organization set. It comprises the set of external elements with which actual exchange relationships are established. The task environment is the most particularized environmental level. The task environment will have the most direct bearing upon the development of the organization. Nevertheless, according to Castrogiovanni (1991), it makes sense to dissect the task environment in greater detail (Figure 3.2).

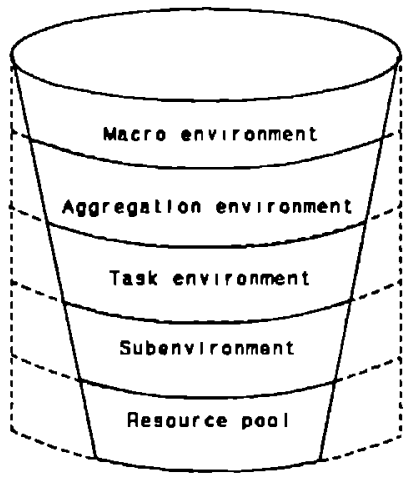

Figure 3.2 Levels in the environment according to Castrogiovanni (1991, p. 544)

Following Lawrence and Lorsch (1967), Castrogiovanni distinguishes organizational subunits. He argues that each organizational subunit concentrates on a different portion of an organization's task environment. As a consequence, inside the task environment Castrogiovanni discerns subenvironments. These are the individuals and organizations who control the inputs and outputs, or resources, that are most relevant to an organizational subunit. In their study, Lawrence and Lorsch identified (a) a market subenvironment relevant to the sales subunit; (b) a techno-economic subenvironment relevant to the production subunit; and (c) a scientific subenvironment relevant to the research and development subunit. The 'lowest' level of the environ- 
ment consists of stocks of particular resources. Studies of how critical various resources are to particular organizations (Pfeffer and Salancik 1978; Pfeffer 1972) are concerned with this resource pool level. This is the most specific level of environment within which the organization operates.

In Figure 3.2, the conical shape is intended to convey the notion that each higher level encompasses more than the one below. This notion is reinforced by the cylindrical shape indicated by the dotted lines. At the bottom of Figure 3.2, for example, the area inside the cone represents a particular resource pool. The area outside the cone but within the cylinder represents other resource pools, each of which can be examined separately. Moving up the cone, a particular subenvironment encompasses two or more resource pools viewed as a composite whole. The area outside the cone but within the cylinder indicates the existence of other subenvironments.

The resource pool level also happens to be the scale at which studies in economic geography and regional economics investigate linkages and information flows. The present study is no exception. Chapter Five will consider the firm-environment interaction with respect to five pools of resources: labour, finances, material supplies, services, and customers. These five were chosen because the field of regional science has developed theories about the importance to business development of each of these resource pools separately. In contrast, empirical studies in organization theory have focused attention primarily on the more abstract macro environment, i.e. the global and societal environment (McDermott and Taylor 1982, 19).

\subsubsection{Environmental dimensions}

A second issue in conceptualizing the organizational environment is the specification of characteristics or dimensions of external elements and their impact on organizational survival. The range of dimensions of organizational environments, as documented by Starbuck (1976), is wide indeed. However, there is an emerging consensus among researchers that there are three basic dimensions to profit-making organizations: munificence, dynamism, and complexity (Dess and Beard 1984) ${ }^{1}$.

\section{Munificence}

Environmental munificence is the extent to which a given environment can support sustained growth (Starbuck 1976). Munificence can be defined either as environmental capacity or environmental opportunity/threat. Environmental capacity or the carrying capacity of the environment is the level of relevant resources present within an environment (Castrogiovanni 1991). Environmental capacity can range from rich to lean. In a rich environment, the organization, like a pigeon, can pick and choose the nicest seeds. In a lean environment, it has to make do with a few grains, for which it even has to fight with competitors. Environmental opportunity/threat is the extent to which capacity is underutilized or overexploited. In other words, environmental opportunity/threat refers to the relative level of resources availability. Environmental capacity and opportunity may not occur always synchronously. For example, places of high-technology industrial concentration are known to have a large supply of hightechnology skilled workers. Nonetheless, enterprises in these areas often face serious 
shortages of this kind of labour. This is because a large local supply may be insufficient to satisfy a larger demand. This results in an 'overheating' of the local labour market, causing severe wage inflation (Oakey 1984). A second example of environmental threat in a high capacity environment occurs when abundant resources are controlled and withheld by a powerful third party. Many oil companies experienced this situation during the oil crises of the 1970s.

\section{Dynamism}

Environmental dynamism is the extent of unpredictable change in the task environment. A distinction can be made between environmental stability/instability and environmental turbulence (Dess and Beard 1984). Environmental stability/instability is the degree of tumover in any of the separate elements of the task environment. With stability, changes arise from the interaction of identifiable component organizations of an organization set. One example might be a fluctuation in the delivery of material input supplies due to the unreliability of a supplier. Sudden shifts in financing policy of governmental or private financiers and strategic moves of competitors may also affect the stability of an organization's task environment. Environmental turbulence, on the other hand, refers to changes which are induced outside the task environment of a focal organization $X$. Environmental turbulence is a result of the increasing interconnectedness of the social world. As a consequence, the relationships between $X$ and the organizations of its task environment are increasingly influenced by forces outside X's task environment (cf Terreby 1968). World-wide changes in technology, consumer affluence, international trade and the mutual interaction of these forces ultimately affect the transactional interdependencies in an organization's task environment. The distinction between environmental stability and turbulence is important. It is related to the degree of organizational autonomy and the ability to plan for the future. Organizations might be able to protect themselves against environmental instability through stockpiling, in-house production, multiple sourcing etc. (Thompson 1967). But hedging against change is much more difficult when it originates in the macro environment (Terreby 1968).

\section{Complexity}

Environmental complexity is conceptualized by Child $(1972,3)$ as "the heterogeneity and range of environmental activities which are relevant to an organization's operations." In other words, the more diverse the relevant environmental activities and the more there are, the higher the complexity (Jurkovich 1975, 382). The number of relevant organizations a focal organization has to encounter is implied by Gerlach and Palmer's concentration/dispersion dimension. This is the degree to which resources are (un)evenly accumulated across societal segments (Gerlach and Palmer 1981). The fewer external elements a focal organization has to deal with, the simpler its environment is. For example, the market environment of a firm which supplies its products to a highly concentrated industry, consisting of only a few very large corporate customers, can be denoted as very simple. On the other hand, an enterprise selling its output to a very competitive industry consisting of numerous small firms operates in a more complex market environment. It has to monitor and anticipate the wishes and requirements of many clients simultaneously. The concentration/dispersion dimension is intertwined with the homogeneity/heterogeneity characteristics of environmental 
factors. This is the degree of similarity or differentiation between the elements of the population dealt with. A homogeneous environment rewards the development of standardized ways of relating to the domain population. It may lead to the development of an undifferentiated set of products or services (Thompson 1967). Homogeneity simplifies organizational activities, because a small set of operating routines may suffice for a large population. Differentiation of consumer demands, on the other hand, forces organizations to utilize knowledge of the desires and demands of a host of heterogeneous clients. Their diversity makes market environments more complex. Table 3.1 summarizes the environmental dimensions discussed above.

Table 3.1 Dimensions and subdimensions of the environment

\begin{tabular}{ll}
\hline \hline Munificence & Capacity \\
& Opportunity/threat \\
\hline Dynamism & $\begin{array}{l}\text { Stability/instability } \\
\text { Turbulence }\end{array}$ \\
\hline Complexity & Homogeneity/heterogeneity \\
& Concentration/dispersion \\
\hline \hline
\end{tabular}

Following Castrogiovanni (1991), we can cross the three mainstream dimensions (Table 3.1) with the environmental levels introduced in the previous subsection (Figure 3.1 or Figure 3.2). This exercise results in a conceptual model of the environment which can be depicted graphically in a two-dimensional (Figure 3.3a) or threedimensional manner (Figure 3.3b).

Having modelled the components and the characteristics of an organization's environment, the question remains whether this is useful in analysing firm-environment relationships in regional science. Before we proceed to assess the relevance of this model, it might be helpful to discuss why organizations interact with their environment at all. Briefly, what is the raison d'être of interactions or relations between an organization and its environment?

\subsubsection{Significance of the environment to an organization}

It is recognized that an organization is not an autonomous entity but instead a unit in interaction with its environment. In this vein, the interdependencies between both entities are given full attention in organization literature. In the wake of the open systems approach, it became clear that the interdependence between the organization and its environment takes the form of service delivery or input-output transactions (Terreby 1968), and that: "much of the stuff that is the object of these transactions falls into the category of scarce and valued resources" (Yuchtman and Seashore 1967, 897). Benson (1975), following Yuchtman and Seashore, assumes that interactions at the level of service delivery ultimately rest upon strategies and processes of resource 


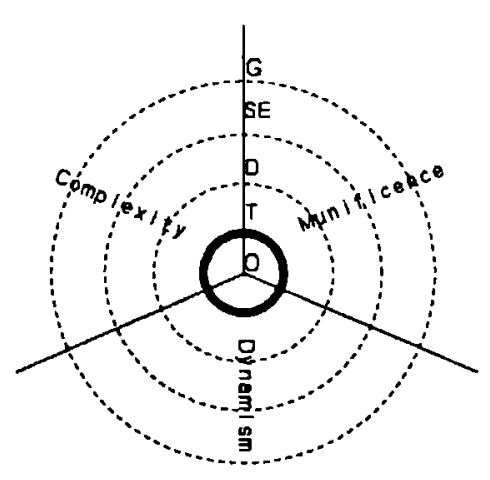

a) Two-dimensional representation

G: Global environment

SE: Societal Environment

D: Domain

T: Task Environment

O: Organization

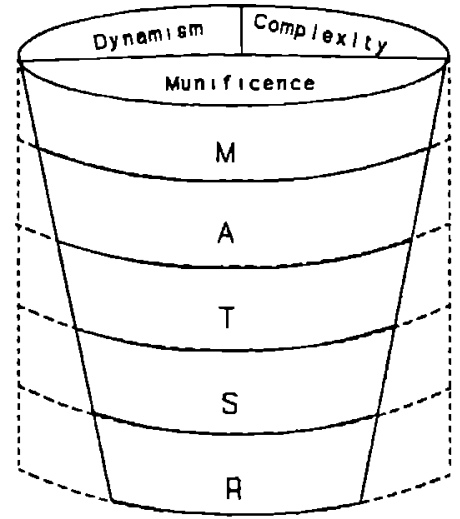

b) Three-dunensional representation

M: Macro environment (=G+SE)

A: Aggregation environment (=D)

S: Subenvironment

R: Resource Pool

Figure 3.3 Two conceptualizations of the administrative environment

acquisition. This means that considerations of resource procurement determine, within limits, the nature of interorganizational relations and transactions. Accordingly, Aldrich $(1979,61)$ states that: "Environments affect organizations through the process of making available or withholding resources, and organizational forms can be ranked in terms of their efficacy of obtaining resources." Furthermore, he notes that even 
theories that are oriented towards decision processes within organizations, and which pay no attention to an organization's environment other than as a source of equivocal information (cf note 1), must view information in view of its relevance for resource acquisition (Aldrich 1979). Hence, we may say that the substance of firm-environment interaction is the obtaining of resources (cf also Van den Bosch 1989).

From this resource perspective the dimensions of an organization's environment can be redefined. They refer to the available abundance (munificence), the variability (dynamism), and the number and heterogeneity (complexity) of resources required for production.

Now, how do these notions derived from organization theory - about the structure of the external environment and the substance of the interaction between organization and environment - accord with the insights from regional science on these matters? This question will be the subject of the next section.

\subsubsection{Spatial and administrative structure of the environment}

We now shift attention from organization theory to spatial science in conceptualizing an organization's environment. In doing so, we agree with McDermott and Taylor that regional science operates within a partially and only poorly developed framework compared with organization theory (McDermott and Taylor 1982, Ch. 3). Regional science focuses exclusively on the spatial dimension of the environment, while ignoring aspatial aspects of external forces. Thus, according to McDermott and Taylor, spatial theory has raised space to the status of the primary and, all too frequently, the only focus of concern. This one-sided view of the role of the environment caricatures the spatial disciplines and obstructs a productive cross fertilization between the insights of spatial and non-spatial disciplines in explaining and understanding economic reality. In this section we will discuss how the adaptation approach within the field of regional science might benefit from making allowance for the conceptual models of the environment shown in Figure 3.3.

Environmental levels in spatial analysis

First, we will consider the impact of taking into account the level at which environmental forces have originated. All environmental forces which impinge upon an organization are transmitted through the same ultimate transformation agent, namely its organization set. Yet these forces may differ greatly in strength and directness. While an organization must comply with some external disturbances, it may be able to influence others. This is affected by the environmental level at which these disturbances originated. Child, for example, states that: "this distinction of several boundaries proceeding 'outwards' from the organization implies that organizational decisionmakers do take positive steps to define and manipulate their own corners of the environment" (Child 1972, 9). Similarly, Terreby (1968) stresses the difference in difficulty of planning and controlling for changes in an organization's task environment. The difference depends on whether such changes were brought forth by the interacting organizations themselves or whether these organizations only transmitted changes which originated in the macro environment. It is not hard to understand that 
changes within the task environment induced by developments in the global environment cannot be influenced by an organization. On the other hand, it may be possible to deal with disturbances which originated within the task environment. For instance, whereas an enterprise is not able to change the ageing of the local population and consequently of its labour market, it might have the power to adjust the curricula of educational agents within its task environment to better suit its demands. Through financing a (part-time) academic chair at a university, through subsidizing a curriculum or academic course, through cooperating with local Employment Exchanges in providing training courses, for instance, enterprises may influence the skills of the labour markets. And while an organization has to take changes in world technology for granted and comply with them, it might be in a position to affect $R \& D$ programmes and production technology of an interacting organization. Actually, this mutual influence or interdependence of organizations is implied by new forms of subcontracting, like co-makership and main-suppliership. These concepts reflect the fact that parent firms are transferring more and more of the responsibility for product development to subcontracting firms. As a result, relations between subcontractors and parent firms may become increasingly equal. Patrick and Rolen (1987) noted this to be the case in Japan over the last 20 years. In sum, we may infer that it is highly inappropriate to treat all external conditions as compelling forces that cannot be influenced or changed. Actually, regional development theory is to blame for this faulty perspective, since it fails to recognize that environmental forces differ in regard to the strength with which they impinge upon organizations. And, as Horvers and Wever (1989) suggest, within limits, the characteristics of the business region nowadays are much less important than economic geographers often assume.

\section{Environmental dimensions in spatial analysis}

The second point to be discussed in the present subsection is an omission in regional science, and particularly in corporate and adaptation geography. These disciplines fail to elaborate on the basic characteristics of environmental elements that affect a focal organization's behaviour and performance. In this context I reiterate the three dimensions of the environment of an organization: environmental munificence, dynamism and complexity. The question can be raised, which of these three attributes is primarily responsible for explaining profit rate differentials and unequal development between regions.

The munificence dimension of the task environment unmistakably bears relevance to spatial research, since the availability of resources can vary markedly across space. Unequal spatial allocation of customers, suppliers, subcontractors, or knowledge is widely held responsible for interregional growth disparities (Aydalot 1988, Malecki 1991, Scott 1988). The uneven distribution of such resources over space makes some locations potentially richer than others. Actually, factor availability lies at the heart of the centre-periphery paradigm and is the cornerstone of agglomeration theory. In contrast, the dynamism of an organization's task environment, or the degree to which environmental factors remain the same over time or are in a continual process of change (Duncan 1972), reveals less clear spatial differences. Factors which cause major changes in organizations' task environments, including institutional developments (e.g. European integration), unpredictable shifts in the economy, increasing differentiation and rapid change of consumer demands, rapidly changing technologies, 
requirements of environmental protection and many others, originate in the macro environment. Thus they are of equal relevance to large bodies of firms, irrespective of their location. Perhaps, due to cultural differences, organizational task environments may be somewhat more dynamic in metropolitan regions than in outlying rural areas. Anyhow, since dynamism or (unpredictable) change contributes to uncertainty (Aldrich and Mindlin 1978), more dynamism in core regions is less of an advantage than a disadvantage of the urban production environment to individual enterprises.

Similarly, the complexity of an organization's task environment is only moderately accounted for by its location. The number and diversity of relevant environmental activities depends mainly on such things as an organization's growth strategy (specialization vs diversification), industrial sector (cf. a mining company, which requires few different inputs, with a micro electronics firm, which requires many different input components), and macro-economic developments (e.g. the growing technological advancement imperative, intensified global competition, and increasing demand for customized products of greater variety and quality). In so far as the spatial setting affects the complexity of the environment - and Scott (1983, 242) argues that spatial proximity stimulates organizations to enter into linkages (disintegration), which subsequently makes the task environment (actual stakeholders) more complex ((though not the domain (potential stakeholders)) - complexity is higher in central areas than in peripheral regions. However, complexity, just like 'dynamism', contributes to (information) uncertainty. This only means that economic conditions are more uncertain and hence less favourable in densely built-up city regions than in sparse peripheral areas.

The conclusion is that interregional differences of organizational task environments are dominated by the munificence-scarcity dimension of the environment rather than by the dynamism and complexity dimensions. Therefore, neither dynamism nor complexity but rather munificence is the key environmental variable in studying firm-environment relations from a spatial point of view. This standpoint is reflected in Figure 3.4 . 


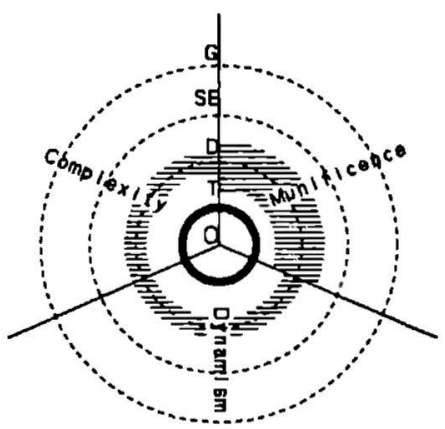

a) Core

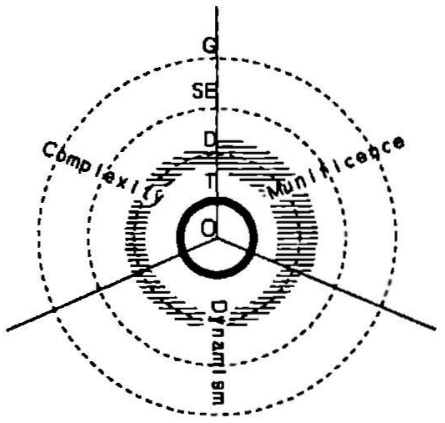

b) Periphery

Figure 3.4 The regional component in an administrative model of the environment

Figure 3.4 depicts the regional content of an organization's environment as a shaded band falling within both the environmental domain and the task environment. On the one hand the Figure highlights the more pronounced regional bias in the linkages of firms located in core areas compared with equal businesses in peripheral regions (see also Figure 3.5). On the other hand the Figure stresses the major importance of the local business region to the munificence dimension and the relatively minor importance of space to the dynamism and complexity aspects of an organization's task environment.

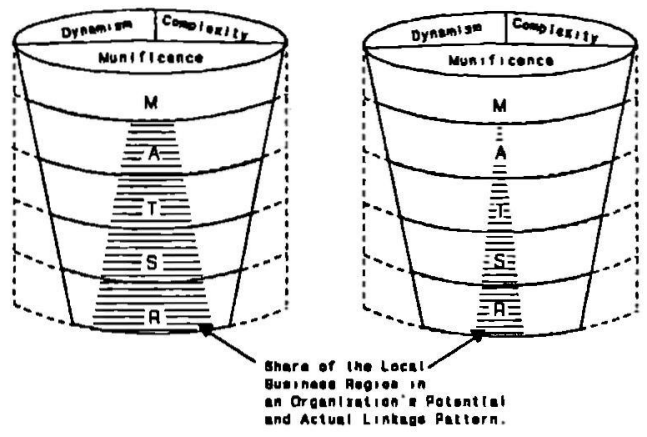
a) Core
b) Periphery

Figure 3.5 The regional component built into Castrogiovanni's environmental model 
This stance is manifested when businesses make location decisions. Firms making such decisions unequivocally choose a location in view of its endowment with the resources needed for production. They do not choose it because, for some reason or other, needed resources fluctuate in one location more than in another. Nor do they choose a location because a greater amount and more dissimilar resources are required in place $A$ than in place $B$. This is reflected in the wider width of the shaded band in the munificence segment compared to the narrower bands in the dynamism and complexity segments.

Within the field of regional science, the ideas of economic development theorists and location theorists clearly correspond to this stance. Their point of departure is (either consciously or unconsciously) the resourcefulness of the environment, unlike adaptation theorists and other corporate geographers. These latter writers, such as Steed (1971), Krumme (1969) and McDermott and Taylor (1982), predominantly focus on organizational adjustment to (macro) environmental change and turbulence, particularly changes in market conditions and technological progress (Krumme 1969). "Correspondingly", they argue, "the optimality of a location for a specific type of activity changes through time" (ibid, 16). This argument, however, has a major shortcoming. It neglects the crucial question of the impact of structural inequalities across space in the general availability of factors of production (core-periphery and urban-rural contrasts) on adjustment processes. Actually, uneven distribution of resources spatially is a rather stubborn phenomenon which may persist over a considerable period of time (Pred 1977). Both Steed and Krumme, on the other hand, suggest that quick successions of environmental changes constantly wash away the locational advantages, including agglomeration economies, of existing centres of production, while creating opportunities for economic development in completely different localities. To illustrate this view, Steed $(1971,324)$ may be quoted: "...locational analysts need to give careful consideration to the changing environment of manufacturing plants and firms, for the original factors underlying a location decision will change with sufficient rapidity that local management soon finds itself confronted by an entirely different set of environmental constraints and contingencies". This contention fails to do justice to longstanding interregional differences in prosperity, which are rather well accounted for theoretically by agglomeration theory.

Just like Steed and Krumme in the 1960s and 1970s, nowadays flexible accumulation theorists like Piore and Sabel (1984), Scott (1988), Schoenberger (1987) and others are concerned with organizational adjustments to the instability of the environment, albeit under a different theoretical flag. The difference is that they do not forget to incorporate the effect of longstanding urban agglomeration economies. This leads these authors to conclude that, when environmental conditions change rapidly, organizations in or near abundant spaces (i.e. dense agglomerations) are able to adjust to uncertainty and instability more efficiently (i.e. at less cost) than those organizations located in lean environments (periphery). The principal flaw in this reasoning is that, without any explanation, it is assumed that enterprises actively seek to deal with macro-economic uncertainty while remaining passive towards scanty resources in the local business region. There is only one possible conclusion from this standpoint: it is that whereas organizations are thought to be able to cope with global uncertainty, they are assumed to be unable to deal with local resource deficiencies effectively. From this conclusion we may infer that if adaptation geographers wish to 
reduce the deterministic overtones of spatial theory, they must focus less on environmental change or contingency than on an organization's dependency on the availability of local resources. What needs to be developed is a model of how (some) organizations are able to cope with scanty factors of production in responding to macro economic forces. Furthermore, such a model has to specify the conditions under which this ability might accrue to businesses.

Searching for some ingredients for such a model, the next section explores the mainstream views in organization theory on organizational action and its determinants.

\subsection{Three perspectives on action}

In administrative science a debate is going on about organizational choice vs environmental determinism or internal adaptation versus external selection mechanisms to explain organizational forms. That debate is lively and remarkably balanced. Historically, the bulk of the literature subscribes to an organizational action perspective, focusing on environmental perception and decision processes within organizations (Hannan and Freeman 1977, 929). However, ecological or contextual approaches gradually came to the fore and received increasing attention in research programmes. These models argue that an organization's situational context determines its organizational structure because of certain, mainly economic, constraints which the former is assumed to impose. In an influential article Child revolted against this "...simple theory" $(1972,2)$ by introducing the concept of 'strategic choice' in organization theory. His contribution was heavily debated and severely criticized by those adopting an 'external constraint perspective'. In this section I will give a brief outline of this debate among organization theorists, based on Pfeffer 1982.

To begin with, Pfeffer adds a third perspective to those two mentioned above. Pfeffer's three perspectives on organizational action are: (a) action seen as purposive, i.e. bounded or intentionally rational, and prospective or goal directed; (b) action seen as externally constrained or situationally determined; (c) and action seen as being somewhat more random and dependent on an emergent developmental process.

\subsubsection{The rational action view}

The rational action perspective argues that organizations act intentionally and rationally to achieve some autonomously constructed goal or value, such as efficiency or higher performance. Though originally focused on intra-organizational behaviour (i.e. the closed-system approach), Child extended the rational action view to organizations' external behaviour. From this perspective, interorganizational transactions are treated as deliberate strategies used by administrators to meet their objectives. Individuals, particularly decision makers, are seen as the basic units of analysis, and their intentions and perceptions form the key variables in organization-environment interactions. Child's strategic choice interpretation of this view perceives organizations as not strictly bound to environments. Child gave three arguments for 
this loose tie between organization and environment: i.e. organizations can make several choices; organizations enact the environment; and managerial perceptions intervene between the organization and the environment.

\section{Organizational choice}

It is assumed that an organization is largely autonomous in its decision-making. Thus, organizational decisions reflect management's preferences rather than environmental pressures. Managerial autonomy may be applied both to ends and means. With regard to ends, it is assumed that goals, such as efficiency or higher performance, are more or less independently constructed. Autonomous choice is also exhibited in the type of activities an organization intends to pursue, i.e. its environmental domain (Miles, Snow and Pfeffer 1974). Thus, to some extent organizations could choose the environment in which they were to operate. Child also noted that, given imperfections in the market, managers could pursue other aims besides maximum efficiency or profits, such as market power or power to control resources. So, the level of performance sought and the trade-offs between performance and other managerial objectives are both elements of intentional and rational action and hence subject to managerial choice.

Considering the menns or strategies organizations employ to attain their goals, even within the same environment there may be multiple responses (or equifinality, in systems terminology) that produce the same basic outcome of successful operation. Miles and co-workers observed two publishing organizations whose top management perceived a great deal of change and uncertainty in their environments. Nevertheless, they made substantially different adjustments to the same set of environmental demands and with the same intended outcome. "One firm went through a major effort to restructure the organization while simultaneously creating a program to financially underwrite a small number of free-lance professionals who were to experiment with new publishing techniques. The other organization, by contrast, retained its current overall organization structure but set up several cross-functional project teams to develop publishing demands to deal with changing environmental demands" (1974, 258). Pfeffer $(1982,160)$ notes that one can employ centralization and personal control or impersonal mechanisms of control; one can use surveillance or develop goal congruence; one can use output control or behaviour control. Thus, according to Pfeffer, there is clearly a range of structural responses available to cope with the internal or external stress faced by organization managers.

\section{Enacting the environment}

In his second argument for a loose coupling between organization and environment, Child pointed out that the environment is not to be viewed as a set of intractable constraints; it can be changed and manipulated through political negotiation to fit the objectives of the top management. Organizations have the power to 'enact' their environment. They create and constitute the environments to which they react. Managers construct, rearrange, single out, and demolish many 'objective' features of their surroundings (Weick 1979). The environment is the output rather than the input of organizational action. As an example, Child refers to the work of Galbraith (1967), who asserted that large businesses are able to create demand for their products and control their competitive environments. In this context Child also refers to Thompson 
and McEwen (1958), who have identified the various procedures which may be chosen in order to assert influence, which the controllers of one organization can exert over their counterparts in other organizations, and vice versa, in support of organizational goals.

\section{Managerial perceptions}

Thirdly, Child argued that the effects of the environment were mediated through the filter of managerial perceptions (see also Koberg 1987). Thus, the linkage between environment and organization was diminished through the operation of perceptual mechanisms and the various biasing effects that occur in such perceptions. This leads to the contention that environmental elements are of no interest in themselves, but are only relevant in so far as information about such elements is attended to by organizational participants. Environments, in the works of such theorists, consist of information about external elements rather than characteristics of the elements themselves. Proponents of this information perspective on environments are Dill (1962), Weick (1979), Lawrence and Lorsch (1967), Duncan (1972) and others.

In sum, strategic choice theory draws attention to decision-makers, their perceptions and their choices. They are regarded as performing a proactive role; their choices are conceived to be autonomous, and their acts are viewed as energizing forces that shape the organizational world. Thus, both organizational and environmental change and variation are brought about by the actors' definition of the situation - the subjective meaning and interpretation that actors impute to their worlds as they negotiate and enact their surroundings (cf Morgan 1992).

In spatial science elements of strategic choice or rational action theory can be found in the behavioural approach and particularly in the geography of enterprise literature. The most striking resemblance between rational action theory in organization theory and geography of enterprise literature is that in both bodies of literature focus on the environment as an output of organizational action.

As already mentioned, Child's article raised lots of dust in administrative science. It was seriously debated by theorists who defended an external control view. They argued that Child exaggerated the degree to which managers of organizations can make a conscious choice in dealing with their environment. Their arguments will be discussed in the next section.

\subsection{2 "The external constraint or situational control perspective}

The second perspective on action, the external constraint perspective, tends to ignore individual or organizational factors, such as personality, preferences, goals and information processing. In this view, action is not seen as the result of conscious foresight and free choice but as the result of external constraints, demands or forces that an organization may have little control over or even no cognizance of. The behaviour of an organization may have little to do with the values and preferences of its decision makers but instead reflect constraints imposed by limited external 
resources. Aldrich (1979) provides a clear example of this dominance of environmental pressure over management's preferences. He notes that, notwithstanding strong preferences among administrators for operating independently and autonomously (administrative rationality), many (smaller) organizations have the best chance for survival (organizational rationality) if they are attached to a dominant association. Similarly, environmental forces can explain the so-called 'paradox of cooperation': businesses entering into partnerships with arch-enemies. A growing number of firms, particularly in the computer and telecommunication industries, fraternally cooperate in technological research and subsequently use the outcomes to wage a life-and-death struggle. These companies work together not because they want to but because they have to. Rapid technological developments, sky-rocketing costs of $R \& D$, and the necessity to become a global player simply command cooperation. A notorious example is the mammoth alliance of the European chips manufacturers called JESSI (Joint European Submicron Silicon Initiative).

Whereas the rational action perspective describes the environment in terms of the information it makes available to organizations, the external constraint perspective conceives the environment in terms of resources (Aldrich 1979, Aldrich and Mindlin 1978). From this perspective the environment consists of scarce resources sought by populations of organizations that compete for as well as share them. Therefore, organizational effectiveness is not expressed in an organization's goal attainment but, instead, in its competitiveness in resource markets (Yuchtman and Seashore, 1967). It follows that according to external constraint theory, the environment is to some extent autonomous vis-à-vis the organization; i.e. attention is focused on the environment as an input condition.

Within the external control perspective of organizations, Pfeffer (1982) distinguishes two variants. The first, population ecology, is a Darwinian evolution model of organizational forms. It deals with the study of populations of organizations and tends to treat the individual organization as a black box. It focuses attention on birth and death processes as these are impacted by environmental conditions. The second variant, resource dependence, opens up the black box of the individual organization. It is concerned with internal adaptations and the politics that occur inside organizations as a response to external conditions.

\subsubsection{Population ecology}

Population ecology deals, as its name implies, with the study of populations of organizations. It is concerned with population characteristics and with variables defined on a population level of analysis. This only means that the stance of population ecology on organization-environment interaction is implicit, not explicit in the model. The population ecology model argues that change in populations of organizations occurs, in part, because of selection processes working on those organizations rather than through processes of adaptation. The importance of selection derives from the fact that there are both internal and external constraints on organizational choice and adaptability (Hannan and Freeman 1977, 1989). The internal factors include sunk costs, leaders' limited information about what is going on in the organization, internal 
politics (e.g. subunit resistance to organizational structural reorganization) and the dominance of institutional norms (Hannan and Freeman 1977, 931). External factors that produce structural inertia include barriers to entry and exit, external constraints upon the availability of information, social legitimacy and uncertainty about the effectiveness of a certain course of action.

Analogous to biological ecology, the population ecology or natural selection model in organization theory has three stages: variation, selection and retention. Variation within and between organizations emerges for one reason or another. This may be either for planned or unplanned (error, chance, luck) reasons. In a seminal article, the evolutionist Alchian (1950) doesn't beat around the bush in presenting his view on rational action. According to Alchian, planned action is meaningless in a world where foresight is uncertain. Organizational success (survival) does not require proper motivation but may rather be the result of fortuitous circumstances. Anyhow, "the population ecology model... is indifferent to the ultimate source of variation, as planned and unplanned variation both provide raw material from which selection can be made" (Aldrich 1979, 28). Once variation has occurred these various organizational forms are selected according to how well they fit their environment, i.e. the survival of the fittest principle. It is important to note that in this selection process, it is the environment which optimizes (Hannan and Freeman 1977, 939). Selection pressures may favour or eliminate entire groups of organizations, such as industries, and the changing population distribution of organizations in a society reflects the operation of such selection pressures. The main selection criteria is whether organizational forms are suited to the resource base of their environment. Selection occurs principally through the competition among organizations for scarce resources. Selection pressures are supposed to be lower in munificent environments than in lean environments. This means that environments richly endowed with resources tolerate more economic activities and organizational diversity than poorly endowed environments. From that it is assumed that "the more numerous and the greater the heterogeneity of variations, the richer the opportunities for an advantageous innovation" (Campbell 1965, 28). In hostile environments, in contrast, organizational diversity is reduced because of intensified competition. According to Pfeffer and Leblici (1973) competition causes increased demand for control. This, in turn, invokes a more structured organization as well as less decentralization and less structural elaboration in the process of product differentiation. In sum, resource scarcity requires tighter controls and therefore a more rigid organizational structure, which, as a side effect, impedes innovation.

The selected form, then, is preserved by so-called retention mechanisms. Retention, the last stage of the organizational change process, involves the maintenance of the organizational form in the future. Retention processes seem to be quite autonomous. Pfeffer (1982, 189) notes that "many of the mechanisms that Hannan and Freeman (1977) listed as impediments to adaptation, are at the same time, mechanisms of retention" (cf the role of organizational routines; Nelson and Winter 1982).

The tight coupling in the population ecology scheme between organizations and environments bears similarity to classic location theory and modern regional development theories. Strictly speaking, in traditional location theory the organization is able to react to changing environmental conditions, but it does so in a very limited way. The only option available to an enterprise to respond to economic conditions is by 
making locational adjustments, i.e. moving to the 'optimal location'. Regional development theory also accords with population ecology in assuming that environmental conditions determine both regional specialization patterns and organizational productivity and profitability (cf. Malecki 1991).

\subsubsection{Resource dependency}

Just like population ecology, resource dependence theory argues that organizations are externally constrained. But the latter theory also focuses on the tactics and strategies used by authorities in seeking to manage their environments as well as their organizations. Because organizations are not internally self-sufficient, they require resources from the environment. Thus, they become interdependent with those organizations and groups in the environment with which they transact. The demands of those in the environment that provide resources necessary for continued survival influence an organization's behaviour in that it must attend to these demands. On the other hand, it is recognized that interdependence can lead to the development of interorganizational influence.

Thus, Pfeffer $(1982,193)$ emphasizes that there are two elements in the resource dependence arguments. The first addresses the issue of external constraint. He argues that organizations will (and should) respond more to the demands of those elements of the environment that control critical resources. As a result, resource dependence theory pays attention to interorganizational power relations, assuming that an organization's position of power affects its activities. The second element argues that, for a variety of reasons, managers and administrators attempt to manage their external dependencies. They do so both to ensure the survival of the organization and to acquire, if possible, more freedom from external constraint ${ }^{2}$. It is even assumed that efforts to manage the environment are directed relatively more toward environmental elements as the pressure they exert on the organization increases (Pfeffer 1982, 199). According to Pfeffer, this is reflected in a firm's board of directors. Pfeffer (1973) found, for example, that the size and composition of hospital boards corresponded with their community context (manufacturing community, agricultural community, and the like). What is more, Pfeffer also reported that hospitals with boards that more closely matched their contextual requirements were better able to obtain additional resources (funds) and grow. Similarly, Pfeffer (1972) found that a firm's capital structure - specifically its debt/equity ratio, an indicator of financial dependence predicted the proportion of bankers or other representatives of financial institutions on its board.

Dorsman and Huizing (1989) suggest that the stronger the perception of environmental pressure in an organization, the more actively it will counteract the environment. Likewise, Staw and Szwajkowski (1975) hypothesized that the less munificent the organization's environment, the more effort the organization will exert to obtain resources from the environment. Their hypothesis was over-supported in that environmental resource scarcity seems to evoke the commission of illegal acts. This is documented by analyses of company and industry data from firms cited for unfair market practices and restraint of trade activities. Whetten (1981) refers to accepted 
principles of organization theory and the common knowledge that innovation lies at the heart of adaptive responses to decline-induced organizational crisis ("necessity is the mother of invention"). Thus, the second part of resource dependence theory traces the various strategy of organizations and their managers to cope with external constraints resulting from resource interdependence. In sum, an organization's behaviour is affected but not determined by the external environment.

\section{Coping with resource dependency}

The list of potential strategies for coping with external constraints and dependencies is endless. In reviewing the literature, it was rather difficult to find a categorization of strategies that cope with the environment that would be applicable to spatial analysis. But Prakken's (1987) categorization of strategies in response to environmental turbulence allowed me to distinguish four broad groups of strategies of firm-environment adaptation. These are: manipulation, immunization, adaptation, and utilization.

\section{Environmental manipulation}

The ability to manipulate or control the behaviour of an interacting organization is largely dependent upon the interorganizational power relations. The parties in a power relationship are tied to each other by the dependence of one on the other, or perhaps by mutual dependence. Note that power is an attribute of the relation between two actors and not of actor A or B taken in isolation (Aldrich 1979). It is possible, therefore, that $A$ may be dependent upon $B$, while at the same time having power over $C$. Furthermore, in the case of mutual dependence, it is possible that $A$ and B may become increasingly powerful with regard to each other. In other words, there is a possibility that increasing interdependence may result in increasing net power. It is this possibility on which coalitions rest (Thompson 1967, 31-32). However, it must be realized that increasing interdependence between organizations means loss of autonomy for each one (Dorsman and Huizing 1989). It is this distinction between power and autonomy which serves to classify manipulation strategies.

Unilateral strategies Organizations acquire and use their power to manipulate environmental elements in a variety of ways. Aldrich (1979) contends that these 'strategies' are preferred according to their impact on an organization's autonomy. The most preferred strategies are those that can be used while maintaining full autonomy. The organization acts without regard to its interacting organizations. Incentive strategies, like price cutting, product differentiation, acquiring prestige, and extensive marketing work through market forces. Other, more direct, inducements to control the activities or relevance of other organizations are unilateral actions, such as cancelling sales or purchase orders, withdrawing support, making donations (including bribery), hiring employees away from competitors, lobbying or going to court.

Bilateral or dyadic strategies A second type of strategies requires cooperation or negotiation with isolated or small numbers of other organizations. Under cooperative strategies, the effective achievement of power rests on the exchange of commitments. 
These strategies are problem-laden, because they involve giving up autonomy to win a greater measure of control over essential resources or an environmental contingency (Aldrich 1979, 295). The most preferred cooperative strategies are dyadic strategies as opposed to action set formation (Aldrich 1979). Dyadic strategies involve cooperation or negotiation with isolated or small numbers of other organizations. Action set formation, in contrast, requires the cooperative interaction of many organizations. According to Aldrich, dyadic strategies are preferred to action sets because the former strategy leaves the interacting organizations more autonomy than the latter. But also within dyadic strategies different degrees of cooperation and commitment can be discerned. Running down the autonomy scale with which the environment can be negotiated in a dyadic context, it is possible to use Thompson's (1967) difference between: contracting, coopting, and coalescing.

Contracting refers to the negotiation of an agreement for the exchange of performances. It is not restricted to agreements formally achieved but may also include informal arrangements to guarantee the provision of essential resources. Contracts may vary from formal long-term arrangements between suppliers and customers to tacit agreements among organizations in oligopolistic market structures regarding their market behaviour (Pfeffer and Salancik, 1978).

Coopting is the absorption of new elements into the leadership or policy-determining structure to be better able to manipulate flows of essential resources. Cooption increases the certainty of future support by the organization coopted. A subtle cooption strategy to exert interfirm control is the establishment of interlocking directorates (USA) and (partial) ownership (Japan) (Aldrich 1979, Gerlach 1992, Glasmeier and Sugiura 1991).

Coalescing refers to a combination with another organization in the environment. This includes joint ventures, mergers and strategic alliances, for instance. These strategies drastically reduce the autonomy of every single member. Therefore they are not pursued very often compared to other forms of interorganizational activities. They are only undertaken to meet severe problems of dependence and uncertainty because of suppliers', distributors', or competitors' actions. In this way market power may be increased, technological developments can be controlled, and so forth and so on (Aldrich 1979).

Multilateral strategies Many sources of interorganizational dependence and uncertainty cannot be dealt with by unilateral incentives and dyadic cooperation. They require the cooperative interaction of many organizations in action sets. An action set is a group of organizations forming a temporary alliance for a limited purpose. Research on action sets examines the purposeful activities of an aggregate of organizations to influence environmental forces. Reciprocity between members is a major problem for some forms of action sets, such as producer boycotts and cartels (e.g. OPEC), as each may fear that the others are taking out more than they are putting into the relationship. Other forms of action sets are joint lobbying, public rallies of small firms and self-employed persons, and consortia. The limited purpose of action sets means that the bonds joining the interacting organizations are usually temporary; temporary coalitions are created to carry out projects no single organization could accomplish. 
It would go beyond the scope of this chapter to illustrate every strategy mentioned at this point. Examples of many of the above-mentioned 'strategies' will be given in Chapter Five, albeit from a spatial perspective. Anyhow, the list of potential manipulation strategies is endless, "limited only by the imagination of managers and employees' constraints on internal variation, and legal and normative sanctions" (Aldrich 1979, 295).

Besides, apart from striving to adapt the environment to the firm's requirements through stronger or weaker external encouragement strategies, an organization may have other options to deal with the environment.

\section{Immunization}

If the environment is too stubborm to be managed by means of inducement strategies, an organization may seek to hedge against external influences. To protect production from environmental threats, organizations may have recourse to several strategies. These are: buffering, rationing, scattering, and vertical integration.

Buffering includes tactics such as stockpiling, but also the accumulation of a nest egg (financial risk reserves or excess resources) to hedge against temporary losses or financial shocks. Stockpiling may be an appropriate and important ploy in massmanufacturing organizations. But it is less feasible when the product is perishable or when the object is inexorably involved in the technological process, as in custom production and consulting activities. Furthermore, in a dynamic market, as currently is the case, stockpiling bears the risk of obsolescence. On the other hand, operating at full capacity (i.e. zero stock principle) makes production processes extremely vulnerable to slight hitches in the supply of even minor components, causing queues, bottlenecks and delays. Thus, operating at excess capacity may eliminate queuing and bottlenecks and reduce unpredictable variations in the time needed to complete each part of the manufacturing process. As a consequence, investing in lots of excess capacity greatly increases delivery reliability (Gertler 1988; Linge 1992; The Economist, August 1990).

Rationing Rationing involves assigning priorities in organizational tasks. An important and well-known example of a rationing strategy is the current back-to-basics trend in private and public organizations. Organizations give new priority to their core activities and externalize non-core activities (disintegrating). Growing international competition allows an organization to hold market leadership or technological leadership in only a very few markets. The current environment is too turbulent and complex for an organization to compete on many fronts. The assignment of priorities in organizational tasks is clearly illustrated in the case of the multinational electronics company Philips. According to Philips's own metaphor, there is a 'Philips tree with components as the trunk and with consumer electronics and information technology and communications as the branches' (De Smidt 1990, 58). Domestic appliances are given less priority, and the company decided to hive off a host of non-core activities completely. These included transport and garage facilities, internal accountancy and assurance services, architectural and engineering departments, canteen services and hotel accommodations, white-goods businesses, diamond tools and many more. The company even decided to sell the vast majority of the activities of the product 
division Information Systems to Digital Equipment Corporation (DEC). After having been engaged in computer production for 25 years, this decision was charged with emotion. However, Philips Computer Systems made huge losses and proved unable to withstand international competition. So, this decision released the organization from serious external pressures and threats, like a lizard that escapes its predators by casting off its tail when attacked from behind.

Scattering Scattering of dependence is the third strategy which helps to make an organization immune to environmental effects. Scattering can be applied to both input and output linkages. Scattering of input linkages is known under the term 'multiple sourcing'. A producer seeks more than one supplier of a component in order to become less vulnerable to the whims of every single one. Scattering of output linkages can be achieved by means of diversification, i.e. the development of new products and acquisition of services. A multiple product strategy can shield an organization from shifts in demand.

Integration The fourth strategy of immunization from environmental pressures is integration or internalization of activities. Integration can occur either internally or externally. Internal integration concerns the in-house development of activities which otherwise would have been contracted out. External internalization relates to the take over of already existing facilities. By internalization, organizations may place the incalculable or failing environment under direct control or become immune to it. Forward integration may serve to improve quality control and protection of the product (for example, bakers owning both a bakery and a baker's shop; computer manufacturers providing software and facilities management to customers), or to gain better control over sales volume and prices (oil-company-owned petrol stations and brewery-owned or financed cafes). Backward integration can make it directly possible for an organization to assure (more regular) supply of materials or components from sources which otherwise might be controlled by powerful firms in monopolistic or oligopolistic markets (for example, oil, paper, and chemicals). Backward integration also makes it possible to improve or secure quality control over parts and components. Horizontal integration is frequently associated with efforts to avoid competition.

(External) integration refers not only to a focal organization's acquisition activities but also to the voluntary merging of this association into a dominant, more powerful organization. As Aldrich $(1979,268)$ noted: "Smaller organizations have the best chance for survival if they are under the protective wing of a more dominant organization." Such reasons underlie the take-over of Volmac, the biggest Dutch computer software company, by Cap Gemini Sogeti (the chief European software company). Volmac sought contact with Cap Gemini after it proved unable to internationalize sales and contacts on its own. Consequently, the company feared ending up in an isolated position in the international world of computer science (Kosterman and Hentenaar 1992).

\section{Adaptation}

Often organizations are not in the position to either influence or hedge against environmental forces. Then they have to accept environmental mandates and comply 
with them. There has been heated debate on the radical changes that increasing customer power and demands have brought about in the way private production takes place in the countries of the Western World. As a consequence of both rising consumer prosperity and growing international competition, fluctuations in market demand have increased, and subtle nuances of taste and need variation in the market place have become manifest (Gertler 1988, Valery 1987). These changing market trends are increasingly forcing firms to abandon mass-production systems and adopt tailor-made and small-batch production systems. Even giant corporations like IBM have to obey these trends and invest heavily in flexible manufacturing systems. They must adopt organizational structures totally different from those prevailing under a mass-production regime (Kelly and Keeble 1990).

The increasing flexibilization of production is an example of internal changes in organizations to comply with environmental mandates. Here, this will be called 'adaptation'. Adaptations, conceived of as intra-organizational changes intended to fulfil external demands, include product changes, process changes, changes in management, and changes in organization structure. It is well known and abundantly illustrated how the transition of rigid mass-production to flexible niche-production changed the nature of products (e.g. smaller components, higher quality, wider product variety), processes (e.g. flexible automation, zero stock production), management style (e.g. shift from autocratic and commanding leadership to participatory and motivating leadership), and organizational structure (shift from centralized and mechanic organization structures to decentralized and organic structures). There is an abundant literature on these subjects in administrative science (e.g. De Sitter 1987, Morgan 1988, Hitomi 1989, Parthasarthy and Sethi 1992).

\section{Utilization}

Finally, organizations do not only counteract the environment, they also make use of it. An organization's employment of given sources of supply is primarily a function of their availability. The presence of the right resources of the right quality is a necessary condition for using them. However, it is not a sufficient condition. Other factors may intervene, such as an organization's ability to obtain the resources required, the organization's need for them and the willingness to use them. The ability and the need to procure certain resources may be affected by the growth stage of the firm. This may hold good for interorganizational variations in the use of external sources of finance within the same environment. Established firms possess a greater ability to raise external finance than new and small ones. Also management's willingness, abilities, and many more factors may intervene between the availability of resources and their exploitation. Variation in the use of external sources of finance, temporary employees, scientific knowledge and other elements may be just as much a result of different attitudes by the owner-managers or of the availability of these resources (Peters 1990). However, this doesn't alter the fact that environmental capacity defines the upper limit at which resources can be used. Hence, it is plausible that, by and large, organizations in rich settings can tap more readily into their environments than organizations in lean environments and thus give the former an important built-in advantage. Table 3.2 summarizes the means discussed above which organizations may use to manage the external environment. 
tble 3.2 Means of dealing with the external environment ${ }^{3}$

\begin{tabular}{lll}
\hline \hline Manipulation & Unilateral incentives & $\begin{array}{l}\text { Indirect: market forces } \\
\text { Direct: non-market forces }\end{array}$ \\
& $\begin{array}{l}\text { Bilateral cooperation/nego- } \\
\text { tiation or dyadic strategies }\end{array}$ & $\begin{array}{l}\text { Contracting } \\
\text { Coopting } \\
\text { Coalescing }\end{array}$ \\
& Multilateral action sets & \\
\hline mmunization & Buffering & \\
& Rationing & \\
& Scattering & \\
Integration & \\
\hline tdaptation & Product & \\
& Process & \\
\hline Jtilization & Management & \\
\hline \hline
\end{tabular}

Reviewing the resource dependence view, it appears that according to this sproach an organization's behaviour is affected but not determined by the external ivironment. With this stance, resource dependence steers a middle course between ganizational primacy in the strategic choice perspective on the one hand and, on the her, the environmental determinism of the population ecology model. Nevertheless, $\mathrm{e}$ instigating and sometimes directing role of the environment in resource dependice theory leads Pfeffer to consider this view as a particular approach within the xternal control perspective of organizations'.

le resource dependence view in organization theory bears some similarities to the laptation approach within the geography of enterprise. First, in describing adaptaon processes in response to environmental constraints or contingencies, both approales see the environment as an input to organizational behaviour and not so much as I output. This is unlike the rational action view in organization theory and corporate sography in spatial science. Secondly, the adaptation approach in geography as well the resource dependence approach in organization theory do not view the organizion as completely powerless or inactive vis-à-vis its environment - as population ology on the one hand and location theory and regional development theories on e other hand actually imply (see Chapter Two). There is, however, one significant 
difference between the adaptation perspective in geography and the resource dependence view in organization theory. This is their conception of the environment. Whereas the former focuses on managing environmental dynamism (uncertainty perspective), the latter concentrates on dealing with resource scarcity (dependence perspective).

Interestingly, rational action theory and external control do not cover all the options. Pfeffer offers another perspective on organizational action, the so-called 'social constructionist' view. I will address this perspective briefly in the next section.

\subsubsection{The social constructionist perspective}

This perspective on action, according to Pfeffer (1982), fundamentally denies either an internally directed or an externally determined rationality of behaviour. In social constructionist approaches, in contrast, it is assumed that much organizational activity is relatively mindless, in the sense that its sensibility is not questioned or even considered, let alone evaluated. Organizational behaviour is conceived of as emerging automatically from and responding unconsciously to the socially constructed reality. This means that social reality is both the context and the result of social interaction processes. Within the social constructionist view of organizational action, Pfeffer discusses three streams of research: paradigm theories, decision-process theories, and institutionalization theory.

\section{Paradigm theory}

Theorists who view an organization as a paradigm posit that an organization's members act according to the shared understanding and the shared exemplars that guide members' actions. "A paradigm is a technology, including the beliefs about cause-effect relations and standards of practice and behaviour, as well as specific examples of these, that constitute how an organization goes about things... paradigms are the glue binding the organization together and differentiating it from its environment and other organizations" (Pfeffer 1982, 227-228). Brown (1978) maintained that the development of shared paradigms is what occurs in formal organizations. Pfeffer (1981a) argued that one of the critical administrative tasks involves the articulation of the organization's paradigm. In this respect Pfeffer refers to Selznick (1957), who noted that a critical leadership task was to provide myths and beliefs that infused day-to-day activity with meaning and purpose.

\section{Decision models}

Decision process models take a bounded rationality view on organizational behaviour. Because it is too costly and problematic to ground decision making in the acquisition of maximal knowledge, organizations economize on information. Thus, the decisionprocess models maintain that the administratively rational thing to do, and what organizations actually do, is to develop performance programs, standard operating procedures, rules of thumb, and so forth, and use these in making decisions. For instance, one commonly hypothesized decision process is that of incrementalism (Padget 1980). Incrementalism economizes on information processing and decision 
time by taking last year's allocation pattern as a base and making marginal adjustments from that base. Decision-process theorists argue that in order to understand a given organization, it is necessary and largely sufficient to understand these performance programs or procedures (Pfeffer 1982, 235).

\section{Institutionalization theory}

Institutionalization theory is the third approach within Pfeffer's class of social constructionist views on organizational action. It addresses the issue of how and why meanings, forms and procedures come to be taken for granted and what the consequences of this institutionalization are (1982, 239). Meyer and Rowan (1977, 341) define institutionalization as "the processes, by which social processes, obligations, or actualities come to take on a rule-like status in social thought and action." For institutionalized acts or institutionalized structures, there is a stable structure of interaction and definition of the situation, which is not necessarily based on rational or instrumental considerations. According to institutionalization theory, actions are not undertaken because they are tied to the achievement of a particular goal (rational action), nor because they are rewarded or enforced by a powerful third party or other intervening processes, but simply because that is just the way things are done. Each actor is motivated to comply because otherwise his actions and those of others in the system cannot be understood. Institutionalization is rooted in conformity, in the taken-for-granted aspects of everyday life (Pfeffer 1982, 240).

According to institutionalization theory organizations import the shared views institutionalized in the environment about what organizations should look like and how organizational work should be performed and incorporate them in their structure as a way to maintain their legitimacy. Organizational and individual action reflect the context of the wider social structure, expectations and norms. As Lincoln, Hanada and Olson $(1981,114)$ noted: "...organizational phenomena are shaped by the cultural values and beliefs, as well as the institutional arrangements, of the populations in which they are embedded... Organizational structures may indeed be loosely coupled to formal goals, but they are closely tied to the cultural and social orientations of the people involved with them."

Ideas from paradigm theory, decision theory and institutionalization theory have a wide applicability. For example, they have some bearing on identifying the sources of competitive advantage of first Britain, then the United States, and more recently Japan during the historic evolution of capitalism (cf Lazonic 1989). Also these theories are relevant to the examination of geographical patterns in cross national merger activities and cooperation between private and public organizations (Molle 1991).

In regional economics and economic geography, several strands of research touch on the ideas of social constructionist theories. They are found, for example, in research on regional identity and spatial images (Pellenbarg 1985) and on cultural and social aspects of new firm formation (De Smidt and Wever 1985, Bleumink et al. 1985, Illeris 1986, Brusco 1986). Also the ideas regarding the impact of selective urban-rural migration of higher-income, highly qualified workers, managers and entrepreneurs on current SME (small and medium-sized enterprise) development in many small towns and rural areas in Northern Europe (Keeble 1989) reveal social constructionist dimensions. Yet, anything like a well-established, clearly recognizable exemplification 
of social constructionist thought is unknown in spatial economics. Indeed, this is a relatively unexplored field, which might account for the many inconsistencies observed.

Pfeffer (1982) notes further that culturally conditioned organizational properties and behaviour need not have any close connection to efficiency or technical rationality. Child (1972), however, stated previously that a theory of organizational structure and action cannot ignore organizational performance The neglect of organizational efficiency or profitability is a major shortcoming of the social constructionist approaches. In this thesis, though perhaps somewhat boldly, I take the standpoint that socially constructed behaviour is merely a particular case of action or adaptation, namely unconscious or mindless adaptation, which could also be pursued rationally. Thus, when I discuss firm-environment adaptation, I do not consider whether the 'strategy' is chosen consciousiy or unconsciously; hence, both types of behaviour are incorporated. Consequently, the following approaches to action are left the strategic choice view, the population ecology view, and the resource dependence view. The next subsection will summarize them briefly.

\subsubsection{The perspectives on action in retrospect}

The main issue addressed in the previous discussion of views on organizational behaviour and performance is whether these are managerially or environmentally derived. The classical stance of organization theory is that an organization's existence, structure and actions are the result of the goals, preferences and perceptions of its leaders. This implies the assumption that managers possess behaviourial autonomy. Presumably, they have some choice with respect to organizational behaviour and design; the range of alternatives is only curtalled by the subjective meanings, interpretations, and the limited information on the part of the management Furthermore, it is believed that strategic choice also extends to the organization's environment. The proactive behaviour of management changes and manipulates the organizational environment to fit the objectives of the organization In other words, primacy is ascribed to organizations in a way that the environment, indeed the whole social world, is seen as an output or derivation of organizational behaviour.

Strategic-choice models have been severely attacked by population ecologists. These theorists emphasize the limits on the degree to which autonomous strategic choice is possible, because of so-called 'internal and external structural inertia'. This means that population ecologists are convinced of the intractability of the environment to manipulation. As a result, according to this view, organizations are placed at the mercy of their environments. They either fortuitously 'fit' into the environment or are 'selected out' and fail Change is explained in terms of an autonomous drift of resources through the economy, rather than in terms of internal managerial action. Population ecology views environments as inputs to organization, not outputs of it. Primacy is ascribed to the environment, which inhibits choice by channelling organizations in predetermined directions. In this sense, the managerial role can be described as inactive or, at most, symbolic in population ecology (Astley and Van de Ven 1983). 
Environmental autonomy is also assumed by resource development theorists, but unlike population ecologists, they do not accept an inactive role or symbolic function of organizational management Because organizations require external resources, they become dependent on certain segments of the environment For a variety of reasons, however, managers and admınıstrators attempt to manage their external dependencies, both to ensure the survival of the organization and to acquire, if possible, more autonomy from external constraint Thus, resource dependency also traces the vanous strategies of organizations and their managers to cope with external conditions In an attempt to create some order out of the chaos of the myriad of 'strategies' that might be undertaken, either consciously or unconsciously, these have been grouped into four categories manipulation strategies, immunization strategies, adaptation strategies, and utilization strategies In a way, the confrontation between the proactive organization in the strategic-choice model and the inactive one in the population ecology model results in a reactive system in resource dependence theory

A major issue, which remains to be discussed now, is whether these perspectives on organizational action are competitive (1 e each one claiming general applicability), or whether they coexist (i e each one dealing with a different portion of the variety of organizations) It has been shown in the previous chapter that regional scientists take the latter standpoint, reserving rational and adaptation approaches for large multiplant enterprises, while applying a selection approach to small and mediumsized firms The last section of this chapter explores whether or not organization theory provides clues for escaping this presupposed deterministic role of size in assessing the adaptability of organizations

\subsection{The role of organizational size}

Aldrich (1979) and Freeman (1982) have implied that the potential to alter the environment to manage external dependence is a capacity possessed primarily by large organizations Thus, they imply that the reactive view of organizational behaviour in resource dependence theory is primarily applicable to larger organizations Also Pfeffer (1982) admits that this view is probably true, taking organizations separately Even Child (1972), who defends a rational action view, reserves the power to influence the conditions prevaling within environments for large organizations Small organizations, according to Child, can only avold unsuitable environments by rationally selecting those types of environment which support the functions the organizations perform The other side of the coin is that, as Astley and Van de Ven point out (1983), the idea of natural selection in population ecology fits small powerless organizations better than big ones

As noted previously (Section 233), the statement that large organizations are more able to cope with their environments than small ones has a major shortcoming The existence of large organızations and their adaptive capacity are taken for granted, without questioning the origins and development of these features As Taylor and Thrift noted "our concern should be with growth and not with size per se" (1983, 451) They quote Penrose (1959), who pointed out that growth is a process, whereas size is only a state Some authors, however, provide inroads to attack the binary 
distinction between size and environmental determinism. This section dwells briefly on these innovative ideas. First, we will offer a critique of the assumption that smaller firms are not able to respond and adapt to environmental conditions. Secondly, with reference to this critique, we will present a model which accounts for adaptation, though not as a static fact but as a cumulative process resulting from dynamic interaction between an organization and the environment.

\subsubsection{The primacy of size attenuated}

Authors who presume that smaller firms are powerless vis-à-vis their environments fail to take into account the following considerations. In the first place, small organizations may develop considerable power by banding together in associations. In this way substantial control over the environment can be achieved (Pfeffer 1982). Wever $(1990,127)$ derived a clear example from the Dutch dairy industry. "For a long time the Dutch dairy industry was dominated by private firms, with milk-producing farmers in a dependent position, because of the perishability of milk. To counter this dependency the farmers established co-operative dairy plants, the first one in Warga in Friesland in 1886. Gradually these co-operatives took over the production of butter and cheese. As a consequence the private companies had to look for other productmarket combinations. One of the alternatives was condensed milk."

So far so good. At the beginning of this century, however, problems arose once more, when the demand for condensed milk increased. As Wever continues, "The private firms had to buy more milk from individual farmers or from co-operatives. They were ready and able to pay a high price for milk, higher than the co-operatives did. This created a problem for the farmers of the co-operatives. They knew from experience that dependency on the private sector was dangerous. On the other hand the milk price could not be overlooked. This conflict between co-operative ideal and individual business was solved by the decision of thirty Frisian co-operatives jointly to start a condensed milk firm: ccFriesland." In this way ccFriesland, now merged into one of the top dairy firms in Holland, was the result of farmers joining forces to fight external threats. By the way, co-operative firms currently run the show in the Dutch dairy industry. In fact, numerous forms of co-operation between small firms can be mentioned, varying from public rallies of farmers, taxi companies, petrol station owners and others to more structural forms of cooperation like cooperations as illustrated above, sharing the same names and logos, syndicates, cartels etcetera.

Secondly, small firms actually reveal higher adaptive flexibility than large enterprises (Rothwell and Zegveld 1982, Pratten 1991). Paradoxically, this statement accords perfectly with ideas of population ecology theory. Remember that, according to population ecologists, the adaptive flexibility of organizations is inhibited because of structural inertia, both internal (e.g. sunk costs, communication structures, internal politics, dominance of institutional norms) and external (e.g. barriers to entry and exit, bounded rationality, social legitimacy) to the firm (see Section 3.3.2.1). In Hannan and Freeman's $(1977,930)$ original formulation: "the stronger the inertial pressures the lower the organization's flexibility and the more likely that the logic of environmental selection is appropriate. As a consequence, the issue of structural inertia is central to 
the choice between adaptation and selection models." The problem is that population ecologists contend that inertia is largely a phenomenon associated with the complex department structures of large organizations (Aldrich 1979), whereas they admit that selection models fit small, powerless organizations better than large ones (Astley and Van de Ven 1983). Betton and Dess (1985) put a finger on this fascinating paradox of population ecology.

The third argument points out the failure of environmental determinists to discriminate between the amount and nature of environmental pressures exerted on small firms versus large ones. Emerson (1962) noted some time ago that the first of two decisive factors of an organization's dependence on some element in its task environment is its need for resources or performances which that element can provide (see Section 3.3.2.1). Thus, a manufacturing firm is first and foremost dependent on a financial organization, to the extent that the firm needs financial resources. It is obvious that a small organization needs less financial capital than a large one. Actually, new firms rely heavily on resources of personal finance (Storey 1982, Donckels 1990). This applies not only to financial resources but also to other factors of production. Peters $(1990,20)$, for example, recognizes that small firms do not require the same large pools of labour and investment; therefore, they can successfully be dispersed to more thinly populated areas. Similarly, Daniels (1982) and Martinelli (1986) argue that large corporate plants use more business services than small firms and are therefore dependent on the centres of such activity. Furthermore, smaller firms place more moderate requirements on the environment, not only in a quantitative but also in a qualitative sense. For example, apart from the fact that these firms cannot afford national or international top managers, they do not need them either. This is because management processes are much less complex in small firms than in large ones. Large multinational enterprises are also highly vulnerable to fluctuations in the exchange rate than small local firms are. This is expressively illustrated by the former president of Philips. He "would like to have the entire range of Philips products produced on the deck of a supertanker. This tanker would than stop off in different countries according to the advantages of moving exchange rates. Philips could then rid itself of one of its biggest worries, extreme fluctuations in exchange rates, which influence sales and profits in unpredictable ways" (De Smidt and Wever $1990,1)$. Similarly, big companies are increasingly scrutinized by society and therefore more susceptible to public opinion and image of the firm than are small ones. Consider also the availability of many types of advanced business services like management consultancy, legal and financial advice regarding acquisition activities, and quotation on the stock market. These and many other resources offered by the environment are of utmost importance to larger multiplant enterprises but are totally irrelevant to small new ones. As a consequence, by virtue of their size, these young companies are also shielded from the threats and contingencies which accompany these dependencies. In line with the foregoing, findings by Peters (1990) about small business growth are elucidating. He observes that high growth led to more demanding requirements for most business inputs. Consequently, rapidly growing companies experienced many more constraints from the environment than slow-growth firms. The former had more labour recruitment problems, difficulties with quality of raw materials, and the availability of technical help. Moreover, they were less satisfied with the services of sales agents and marketing consultants. Peters states that 
constraints mainly follow from growth; "Most of the consequences of growth were in fact likely to be growth-induced constraints."

In short, small firms, almost by definition, maintain a smaller and less complex task environment than large ones. Consequently, the former are exposed to fewer (potential) pressures from the environment and therefore have to generate less counteractive power and adaptive capacity than the latter to bring firm and environment in line with each other. The conclusion is that it is not an organization's absolute power but rather its strength in relation to the size and complexity of its task environment that counts in dealing with firm-environment adaptation. An absolute notion of organizational adaptability and power vis-à-vis the environment may reveal a clear gap between small and large organizations. However, starting from a relative notion, this gap may be much smaller and less self-evident.

\subsubsection{Adaptation development}

This relative idea of adaptability does not alter the fact that, as a prercquisite to growth, organizations have to enhance their ability to deal with environmental conditions, both threats and opportunities. This focuses attention on adaptation as a dynamic process that is the result of the relative strength and power of an organization in relation to its environment (Hrebiniak and Joyce 1985, 347). Chakravarthy (1982) developed a model which conceives of adaptation as a development process. For that purpose he brought together four different concepts of adaptation. These concepts are: 'state of adaptation', 'adaptive ability', 'adaptive fit' and 'the process of adaptation'. Figure 3.6, page 76, depicts Chakravarthy's model in an adapted and simplified form.

\section{State of adaptation}

The state of adaptation of a business organization is the level of adaptation in which it can survive the conditions of its environment. Each level describes the extent of environmental complexity that can be dealt with. And the more environmental complexity that can be handled by a firm, the better are its chances for long-term survival. Chakravarthy differentiates between three states of adaptation. These are defined as: unstable, stable, and neutral.

In the unstable state, a firm's adaptation to the environment is extremely fragile and vulnerable to changes in the environment. The manager of such a firm deliberately creates stability through a series of decisions which lessen the organization's interactions with its environment. It is possible for a firm in this state of adaptation to show good financial results in the short run. However, its long-term viability is severely constrained. As mentioned above, limited integration with the environment is a feature inherent to small organizations, rather than large ones, and thus are they relatively sheltered from a host of environmental pressures.

A stable state describes the state of adaptation in which instead of protecting itself from the environment, the firm is open to it. The firm reacts to environmental changes and complies with environmental mandates. In a neutral state, a firm can withstand most environmental changes because they have been anticipated before 
their occurrence and the firm has invested in the requisite adaptive ability. The environment may even have been modified to suit the organization's needs. These organizations are continuously searching for market opportunities. They often create changes in their environment, to which their competitors must respond.

Although all three states of adaptation, within limits, are viable ways of coping with the environment in the short term, they do not have the same immunity from environmental constraints in the long run. According to Chakravarthy, the neutral state has the highest immunity, followed by the stable and unstable states. A firm seeking to ensure its future should prefer a neutral state of adaptation over the other two states. However, the state of adaptation that a firm aspires to is predicated on the resources that it commands, that is, its adaptive ability.

\section{Adaptive ability}

An organization's adaptive ability or capacity consists of two basic components; material capacity and organizational capacity. The material capacity of a firm includes its material resources, i.e. equipment and financial capital. This material capacity sets the upper limits to the strategic choices. When the material capacity of a firm is poor, the strategic choices open to the firm in order to deal with the environment are both limited and conservative, i.e. directed at conserving its limited resources. In contrast, a firm with unlimited material resources can explore several strategic actions.

A firm's organizational capacity describes the quantity and quality of its commercial, technological and human resources as well as the organizational structure. Actually, a firm's organizational capacity contains all its non-material assets, including its know how and its creative and innovative capacity (Van der Zwan 1990). In Figure 3.6, material and organizational capacity are assumed to relate mainly, though not exclusively, to organizational size and age respectively. Whereas at its start the organization already has a certain, albeit limited, capacity (both material and organizational), namely the founder's own property as well as his abilities and ideas, the organizational features of size and age only come into existence after it has been started up. This fact is flagrantly overlooked in new firm research, which, by implication, treats all new firms alike as tabula rasa, being unknown quantities and fully dependent on the environment for their resources. Nevertheless, it is just this preexistence and start-up capacity which constitutes an important determinant of the early growth potential of a new firm.

In order to withstand environmental pressure and to stay in business, a minimum of acceptable material and organizational capacities is required. These minimum requirements reflect the pressure on the organization exerted by the environment. It can be seen in Figure 3.6 that along with the growth and maturing of the organization, the material and organizational capacity of a firm increase, as well as the pressure exerted from the environment.

\section{Adaptive fit}

Chakravarthy's third concept, adaptive fit, must be distinguished from state of adaptation, referred to above. "Whereas a state of adaptation ensures survival, an adaptive fit ensures in addition the optimal use of the material and organizational capacity of a firm" (1982, 40). A state of adaptation can represent several strategies, with varying emphases on creativity and productivity. Yet an adaptive fit represents 
an optimum between creative (i.e. effective) and productive (i.e. efficient) use of organizational resources, both material and organizational. In Figure 3.6 all firms are adapted to their environment effectively, but only the firms A1, B1 and $C 1$ are adaptively fitted. According to their state of adaptation, A1, B1 and $\mathrm{C} 1$ are in an unstable fit, a stable fit, and a neutral fit respectively. The most interesting and crucial point in Chakravarthy's model is that firms can move both within and between the different states or levels of adaptation. They do so through the process of adaptation.

\section{Process of adaptation}

According to Chakravarthy, "The process of adaptation includes two subprocesses: adaptive specialization and adaptive generalization. Adaptive specialization is the process of improving the goodness of fit in a given state of adaptation. It refers to the rationalization of processes and structure using available material and organizational capacity for moving to the nearest adaptive fit. Adaptive generalization refers to the process that improves the survival potential of the organization. It is the aim of adaptive generalization to enhance the material and or organizational capacity of a firm as required to move it to the next higher state of adaptation" (1982, 41-42).

In order to understand adaptive generalization, Chakravarthy $(1982,42)$ considers a successful firm to be adaptively fitted with its environment. "In such a condition of fit, the firm generates a surplus of contributions over the inducements that it provides. This surplus is called 'slack' (Cyert and March 1963)". This slack can be invested in improving both the material and organizational capacities of the firm. Through these investments, a firm can consider new strategies for coping with greater environmental complexity and dependence. Once a firm has reached a higher state of adaptation, it may seek adaptive fit in keeping with its new level of resources (adaptive specialization). In this way Chakravarthy's model views the ability to adapt not as a given capacity but as a dynamic process, resulting from the cumulative interplay between a given level of organizational adaptive capacity, environmental forces and organizational adaptation processes. I agree with Hrebeniak and Joyce who state that: "it is necessary to think in circles (Weick 1979), to investigate the reciprocity of relations between organization and environment, and to study the mutual causation that obtains" $(1985,347)$. Chakravarthy's conceptual framework can be used to describe how the adaptive capacity and size of a small start-up business may unfold and develop in the course of time. The model can describe appropriately how a small, new-born business might exploit and expand this adaptive capacity. Beginning with a given, albeit modest, amount of adaptive ability (cf the white triangle at the lower left in Figure 3.6) - the origins of this important initial capacity will be addressed in Chapter Seven - the firm will move to higher domains and scopes of power vis-à-vis the environment. 


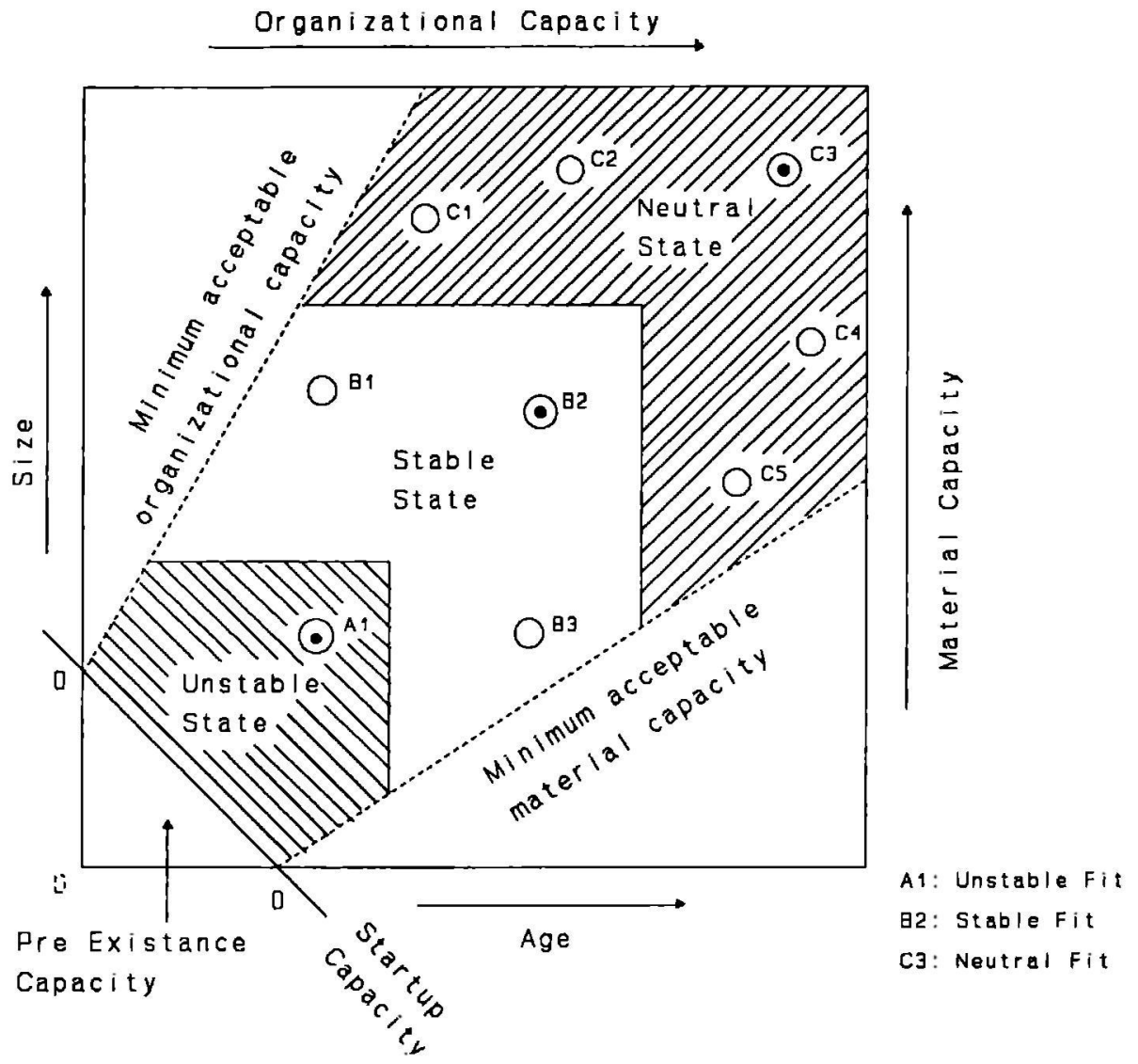

Figure 3.6 Adaptive abilities, state of adaptation, and adaptive fits (adapted from Chakravarthy 1982)

\subsection{Summary}

The aim of this chapter was to find out whether organization theory provides clues to creating more leeway for entrepreneurial action vis-à-vis the spatial context. This could be useful to a theory in spatial science which seeks to explain the phenomenon of small firm growth in contrasting environments, i.e. both rich core regions and lean peripheral areas. We sought to answer three questions.

(1) How is 'the environment' conceptualized in organization theory, and how can this be of use to regional science? 
(2) How is the interaction between organization and environment perceived in organization theory, and how can this be compared to interpretations in spatial theory? In other words, where do we currently stand in the debate about organizational adaptation versus environmental determinism in organization theory as compared to spatial science?

(3) How do organization theorists perceive the intervening role of organizational size in the interaction between organization and environment? In more concrete terms: does organization theory provide any theoretical notion to break through the assumption of the predominant influence of size on the dichotomy between organizational adaptation and environmental determinism?

Castrogiovanni views the environment as being composed of environmental levels and dimensions. Environmental levels refer to the directness and strength with which external forces impinge upon an organization. This distinction of several boundaries proceeding 'outward' from the organization implies that environmental influences cannot be generalized and that a distinction must be made between more and less compelling forces. Environmental dimensions - munificence, dynamism and complexity - are specific aspects of the environment to which organizations respond or by which they are affected. Formulated in terms of positive action, the dimensions capture why a resource is sought (Aldrich 1979, 62). Applying this formulation in the sphere of spatial science, the question becomes why organizations may prefer one location over another. It is unequivocally not because of dynamic or complexity aspects of the local task environment but because of munificence aspects. This illustrates the key function of the munificence variable of the environment in spatial science. Adaptation geography forms no exception. So, the rmison d'être of adaptation geography must be how organizations respond to resource scarcity or munificence and not to turbulence or heterogeneity. Hence, adaptation geography should explicitly incorporate organizational targeting of resource dependence in dealing with information uncertainty.

Regarding the second question about the balance in organization theory between organizational and environmental primacy, three standpoints can be identified: a) the organization proactively moulding the external environment (i.e. rational action/strategic choice perspective), b) the environment selecting organizations (environmental selection/population ecology view), and c) the organization reacting to external conditions, being affected but not determined by the environment (resource dependence theory). Stretching the imagination a little, these viewpoints also can be identified in spatial science. Whereas in spatial theory, environmental determinism predominates over theory development, in organization theory the balance between different approaches is more in balance. The conclusion is that organization theory's resource dependence perspective provides useful clues and insights for developing a framework. That framework makes it conceptually possible that organizations respond actively to external conditions of the local business region and handle locational disadvantages effectively. These insights revolve around the notion of the reactive orgnnization as well as around four basic means of responding to environmental forces: manipulating, immunizing against, adapting to, and utilizing the environment. It is the aim of this study to develop adaptation geography further, while using these insights derived from organizational resource dependence theory. 
The third question concerns the issue of whether an adaptation approach in geography must be reserved for a small elite of big powerful companies, or whether it can also be applied to the remaining 70 to 90 per cent of firms, made up of small and medium-sized enterprises. Although small firms possess less power to deal with external forces, they also place fewer requirements on the environment and hence are exposed to fewer external pressures than big enterprises. Irrefutably, there exists a wide gap in absolute power between large multiplant companies on the one hand and small single-plant firms on the other. Nonetheless, the relative gap may be much smaller. If the adaptive capacity of small companies exceeds a minimum level they may be well able to cope with the environment in accordance with their needs for survival. I proceeded along the lines of a development model of organizational adaptability, designed by Chakravarthy (1982). Chakravarthy's framework describes how a modest initial adaptive ability of organizations can be expanded along and in interaction with the organization's size. Meanwhile, the organization will place evergreater demands on the environment and consequently experience ever-increasing external pressures.

Using the insights from this chapter as a point of departure, in Chapter Five I will illustrate how small, successful companies in the rich Dutch urban core region (Randstad Holland), as well as in less well endowed peripheral and semi-peripheral areas, deal with both the opportunities and the constraints of their local business region. The examples are taken from my own in-depth research conducted among 13 small and medium-sized companies. Most of these are young, high-technology enterprises, operating both in the industrial and in the service sector (Vaessen 1989, Vaessen and Wever 1990). The research is characterized by a qualitative research method. This methodological approach is not without criticism. So, before I proceed to discuss the nature and development of the responsiveness of these enterprises towards their local business region, I will first address some methodological aspects and explain the selection of the businesses investigated.

\section{Notes}

1 Distinguishing these three dimensions of the environment gives rise to two different perspectives on firm-environment interaction. The munificence dimension directs attention to the dependence of an organization on external resources. Environmental dynamism and complexity, on the other hand, direct attention toward lack of information and resulting uncertainty. Both perspectives indicate that dependence and uncertainty are key concepts in the explanation of firm-environment interaction (cf Aldrich and Mindlin 1978, Douma and Schreuder 1992).

2 Hannan and Freeman (1989), subsume the resource dependence theory under the class of adaptation theories.

3 As discussed above, organizations can deal with their (aggregate) environment in four ways: manipulation, immunization, adaptation, and utilization. Although these ways have been presented as analyt cally distinct methods, reality is often more obscure. Actually, these methods are inextricably intertwined and ambiguous. For example, a firm that tries to manipulate the market by enhancing consumer satisfaction first has to 
improve the adaptation of its product to the market's desires. Similarly, organizations that seek protection from the national government against foreign competition (immunization) often first have to mobilize the state to intercede (through negotiating, lobbying, making threats, and so on) and to take appropriate policy measures (manipulation). The web of means of coping with the environment may be much more intricate yet. Before Philips could withdraw from the competitive pressure on the computer market (i.e. immunization), the company first had to reorganize its computer division (adaptation) to make it fit for sale and to induce potential acquisitors to take over (manipulation). Subsequently, Philips entered into talks with Digital and after a year of tough negotiations the company succeeded in selling its computer division to Digital (manipulation). 


\section{Chapter Four}

\section{Research method and sample}

\subsection{Introduction}

Chapter Two recorded how spatial theorizing in general and modern regional development theory in particular failed to incorporate the entrepreneurial factor in an adequate way. In Chapter Three, we put our ears to the ground of organization theory, seeking clues for improvement. The present chapter explores ways to tackle this subject matter methodologically. How can one convincingly highlight organizational autonomy vis-à-vis the environment within a prevailing picture of centreperiphery and urban-rural contrasts? This chapter is divided into two parts. The first part (Section 4.2) considers the research method used; the second (Section 4.3) reports on the selection and description of the sample.

\subsection{A qualitative method}

Getting straight to the point, the present thesis is based on a somewhat unusual, though not new, research method in regional science, a qualitative research method. This involves the collection and analysis of qualitative data. Barton and Lazarsfeld $(1961,95)$ define qualitative data as "detailed, concrete, non-metric descriptions of people and events". The distinctive difference between quantitative and qualitative research is that the latter kind is strongly focused on obtaining in-depth knowledge about the nature of a particular phenomenon, whereas the former is much more oriented towards gaining insight into its representativeness (Maso 1987, 30). Of course, there is not only one qualitative research method. Actually, qualitative analysis can be pursued in many different ways, and every method is subdivided into many different variants, depending on the specific context. The following sections address the use of a qualitative approach in the present research as well as matters of reliability and validity.

\subsubsection{Features of the research method}

The first distinctive characteristic of the project's research method is that an in-depth approach has been applied, resulting in case studies of individual enterprises (cf. 
approach has been applied, resulting in case studies of individual enterprises (cf. Vaessen 1989, Vaessen and Wever 1990). Though the choice for a case study perspective is a rather unconventional way to study small and medium-sized firms in regional science, it is easy to defend. The task of the project was to fill in a void in spatial theory; that is, to explain (modem) small-firm behaviour outside of metropolitan core areas. This task required an explorative approach. In other words, the research was expected to produce one or more hypotheses to fill this vacuum. And as Platt $(1988,8)$ notes: "it is universally recognised, even by those who scarcely allow it any other role, that a case study may suggest hypotheses, interpretations, empirical uniformities for future (quantitative) investigation. It does so by showing that things are so, or that such an interpretation is plausible, in the particular case, so that they might also be so in other cases" (cf also Wester 1987).

The inclination to conduct case studies is reinforced by a growing dissatisfaction on the part of the author with the rough and vague information provided by the prevailing quantitative methods. The ready-made choices in structured questionnaires and the measurement of statistical associations in conventional methodology drastically abridge the intricate and multidimensional nature of the everyday world. Consequently, any appreciation of the adequacy and the accuracy of the asserted relations disappear from view. Too frequently the importance of locational conditions for growth is postulated without showing the underlying mechanisms. Case studies are assumed to give insight in these matters, because they can provide material which is inaccessible or less easily obtained by other measures. Platt $(1988,11)$ refers to this as the function of micromediation of case studies.

A second feature of the qualitative research method used here is its comparative perspective. Thus, unlike case study research using but one case, the present research is based on several cases. The main reason for multiple cases is to be better able to control for the impact of the quality of the production environment. Thus, behaviour of favourably located firms is compared with the actions of less favourably sited businesses. In the Dutch context, it is assumed that the strongly urbanized polycentric metropolis in the western part of the country, known as Randstad Holland, offers a more favourable production environment for many types of modern and highly valued economic activities than do other regions. As a consequence, the present research investigates the firm-environment relationship of Randstad firms in comparison with non-Randstad firms. In this way the research counters an important objection aimed at corporate geography, namely that it is essentially a single-firm or at least a single-region approach. Accordingly, corporate geography ignores the effects of comparative (dis)advantages on interfirm competitiveness and development. In order to put forward hypotheses more confidently, several firms from both types of area, central and peripheral, have been investigated. However, the number of cases should never exceed the quantity which would make it impossible to retain the individuality of each case. Ultimately, 13 firms have been selected for investigation.

A third hallmark of the present research is its non-interpretative nature. Often, qualitative research is considered to be interpretative research. Eyles $(1988,1)$ even suggests replacing the designation interpretative geography for qualitative geography. Applying an interpretative approach means that researchers try to perceive the world of individuals and groups as the subjects see it. The task of research, it is said, is, therefore, to uncover the actor's view on and perception of the social world and how 
this relates to behaviour. This would imply, in the end, that the researcher's hypotheses or theories have to correspond with the respondents' opinions. Not so in this study. Although this research deals with entrepreneurial behaviour it does not focus on understanding the underlying reasons, but on recording actions and assessing their influence. Remember that the present study is aimed at explaining small-firm success in different spatial settings. Within this context it is largely irrelevant what process of reasoning or motivation drives organizational behaviour towards the environment. The only thing that matters is whether or not the organization's actions contribute to its competitiveness and survival. What is interpreted in this research is how an organization's behaviour affects its relation to the environment in general and to the regional setting in particular. From this an assessment is made about the relative weight of the contribution of the regional production environment to the firm's success and survival. So, unlike mainstream qualitative research, the present approach views the entrepreneurs or businesses investigated as objects of research and not so much as subjects.

The fourth and last pertinent feature of the research method is that it adopts a longitudinal approach. This means that the research is processual and change-oriented. Actually, it comes down to reconstructing a firm's behaviour towards the spatial environment from its foundation until the moment it is investigated. This is necessary because it can be hypothesized that the role of the regional environment is most apparent in the early growth stages of the firms. Admittedly, this was not realized from the outset. Somewhat naively it was expected that a random indication of linkage patterns and an assessment of the entrepreneurs' explanations of their success would yield useful material. Supposedly, this would lead to insight into the significance of the quality of the production environment to small-firm performance. However, soon after the field work started, it appeared that a longitudinal approach would be indispensable. Even businesses which were hardly interwoven with their local business region at the time of the investigation, have benefitted from their surroundings during earlier growth stages. Such items as the career record of the entrepreneur and the foundation and early development of the firm, were initially brought up only for the sake of formality. Yet these points became the focus of attention as the research proceeded.

Summing up, the research on which this thesis is based can best be typified as a qualitative, though objectifying, comparative case-study approach with a longitudinal character and an explorative assignment. That is quite a mouthful. How has this task been realized?

\subsubsection{Putting the research method into practice}

The collection of the data

The data which meet the above requirements of the research method are primarily obtained from lengthy in-person interviews with the directors of the firms. These were informal or semi-structured interviews. Unlike structured or formal interviews, the questions asked in informal interviews have a sequence and wording that are not standardized and worked out beforehand. In semi-structured interviews, the inter- 
viewer tries to tailor the wording of the questions to each particular individual and ask the questions in an order appropriate to the interviewee. The researcher does not, however, approach the task without any framework. There is usually a checklist of topics to be covered by all respondents. Such interviewing requires great skill on the part of the researcher. That individual must not only be an attentive listener but also a good conversationalist, able to keep a dialogue going. We will return to these points in the discussion about the reliability of the results.

Anyhow, the interviews took place in two rounds. The first round of interviews were conducted early 1988, the second round two years later. The first series of interviews was aimed at gathering as much information as possible about the relation between the firm and (certain elements of) the environment. In the second series of interviews, the questions were much more purposeful. We will first address the 1988 interviews.

Because of the absence of an elaborate pre-existing body of theoretical work about small-firm growth in peripheral areas, which can be used as a starting point for analysis, it was decided to formulate the research question in an open and explorative manner. Accordingly, the research question was wide-ranging, directed at collecting large quantities of information about the firm's performance in relation to its place of location. Three kinds of questions were posed, dividing the conversations roughly into three parts.

In the first part of the interview we inquired about particular events in the firm's existence. These questions involve the previous career record of the entrepreneur, as well as the company's start-up (why, where, when) and development (shape of the growth curve, particular breakthroughs, important events and developments with respect to product-market combination, and other significant occurrences in the history of the company). The main goal was to identify external links of influential events and to unravel the spatial structure of these relations. For example, if a respondent mentioned an innovation during the conversation, I would then ask how the original idea was born, who was involved and where they were located. I was also interested in whether or not external agencies are called in to assist with the implementation. This style of questioning, which is labelled haphazard interviewing (Wester 1987, 34), requires great alertness on the part of the interviewer.

The second part of the interview was designed to provide insight into the general state of affairs in the relation between the firm and its environment. Five important environmental elements or external resources, drawn from the literature, were considered. These resources are: labour market, financing opportunities, material supplies, supporting services, and market opportunities. We were primarily interested in the geographical structure of these relations, their dynamism, and their causal mechanisms. Thus inquiries had to be made about the ratio of internal to external deliveries, the action radius of external linkages (i.e. regional ${ }^{1}$, national, international), and how these linkages came into being. The third and last part of the interview contained some questions about the entrepreneur's viewpoint; how does he explain the firm's success and how does he perceive the role of the production environment?

I compiled the profiles from the conversations with the entrepreneurs but also used information from annual reports as well as newspaper and magazine articles in which their businesses are mentioned. These sources often contained useful clues for the interviews. 
The analysis of qualitative data

The interviews were tape-recorded and subsequently transcribed This yielded about 330 pages of text. The processing of the empincal material gathered in the first round of interviews went as follows Five interviews were selected to prepare an equal number of profiles, which depict the firm-environment interaction in detall. These profiles have been published in Vaessen (1989, Ch 3-7) These 'company biographies' represent the first step in attempting to understand the role of the environment in disparate regions The other eight firms are systematically compared to the five companies which have been portrayed in detail (Vaessen 1989, $\mathrm{Ch}$ 8) Here we analyse this matenal and draw some prelımınary conclusions (Vaessen 1989a, $\mathrm{Ch} \mathrm{9,1989b)}$ This exercise yields insights into the relevant research issues for explaining small-firm growth under different locational conditions Among other things, it elucidates a firm's behaviour in response to the avallability and suitability of external resources as well as the conditions of imtital rapid firm growth

Then, early in 1990, two years after the first interview had taken place, the opportunity arose to visit five firms for a second time This opening occurred within the scope of writing a book about those firms (Vaessen and Wever 1990) On the second occasion I gathered additional information to buttress the central themes that had emerged in the initial analysis Then, after the book was finished, the observations were re-examined from a theoretical perspective What basic processes are really involved, and what are the key issues? In this alternation and interaction of data collection and analysis, the research questions relevant to growth in contrasting environments gradually became clear I re-analysed the interviews and profiles in the light of new, more precisely formulated questions The present volume is the account of this more fundamental reconsideration

The cyclical procedure explained above is a distinctive feature of explorative qualitative research (Wester 1987, Maso 1987, Strauss and Corbın 1990) In taking such an approach, the researcher has to go through the empirical cycle several times In response to an approximate problem, data are collected, analysed and evaluated tentatively. Then, the first general question is narrowed down and the data are analysed again If initial information is not satisfactory in vew of the adjusted research question(s), the investıgator may even have to return to the field and collect new or additional data before he can proceed with the analysis In this way, one cycle follows the other

\subsection{Objectivity of qualitative research reliability and validity}

It must be noted that a qualitative research method as explained above is not beyond criticism Its major drawback concerns the impossibility of measuring the reliability and validity of the findings How can one determine whether the outcomes of qualitative research are tied to the idiosyncrasies and personal views of the researcher or intervewer, for example? More than once, qualitative research has been taken to task for its subjectivity and arbitranness (Swanborn 1990) And how about its validity? What can be proved on the basis of a close examination of only a few cases? Unlike quantitative research, qualıtative analysis is not able to present exact calcula- 
tions and standards on these matters. However, this does not imply that qualitative research is completely inadequate and that 'anything goes'. First we will deal with the question of reliability, and then discuss the validity of the present research.

\section{Reliability}

The problem of reliability is concerned with the control over and/or measurement of unsystematic research errors. Reliability may be defined as: the extent to which an experiment, test, or measuring procedure yields the same results on repeated trials (Webster's New Collegiate Dictionary). Swanborn (1990) considers those research outcomes as reliable which remain stable under replication of researcher, instruments and circumstances. All three aspects will be discussed below with respect to the present research.

Reliability of the researcher If the same investigation would have been conducted by another researcher, would he had achieved the same results? In answering this question, which points to the problem of the so-called researcher's bias, we would like to address two forces which might have influenced the outcomes and conclusions of the research. These are the experience and skills on the part of the researcher in conducting in-depth interviews, and the researcher's foreknowledge and interests.

On aspect of researcher bias with the potential to influence the research outcomes is the extent of the researcher's previous experience in conducting interviews. I myself had some experience in conducting unstandardized in-depth interviews in a research context before the start of the present project. To further minimize the researcher's bias in this respect, some additional measures were taken. It has been noted earlier that unstandardized interviewing requires great skill on the part of the researcher, who must not only be an attentive listener but also a good conversationalist, able to keep a dialogue going. Regarding the aspect of careful listening, it was beneficial that the interviews were recorded on tape and transcribed literally, which was, by the way, a very time-consuming job. Tape-recording turned out to be particularly useful for detecting inconsistencies and partial answers of the interviewees which had been missed during the sessions. These inaccuracies have been corrected partially in the second round of interviews and, occasionally, through an additional telephone call with the entrepreneurs.

With respect to the ability to keep the dialogue going, it was of great help that I supplemented the standard interview checklist with topics specific to every individual firm. These have been derived from annual reports as well as newspaper and magazine articles in which the businesses are mentioned or portrayed. Furthermore, I underwent a steep learning curve from one interview to the next. In sum, I feel that, due to the measures taken and the rapid learning effect, even more seasoned in-depth interviewers, all else being equal, would not have achieved different results. At most is it possible that a more experienced interviewer might have been able to buttress the conclusions more thoroughly.

What might have influenced the outcomes and conclusions of the present research more significantly are the foreknowledge and/or interests of the researcher. The first round of interviews was aimed at obtaining information about a wide range of aspects on firm-environment relationships, in order to optimize the chance of detecting clues for the formulation of hypotheses. Therefore, the data probably 
contain indications worthy of further investigation However, not all of these signs may have come to the forefront Some have simply been overlooked by the researcher, whereas others were noticed but underestimated The information selected for further elaboration may have been influenced by the researcher's idiosyncrasies, such as his foreknowledge and interests.

Thus, the present research elaborates on the observation that the enterprises located outside of the Randstad, from the outset, sold their output mainly beyond the local business region on a national and even international scale. This is diametrically opposed to the regional orientation of the Randstad firms in the sample as well as to what is known from the literature Though I did not fall to notice this duning the fieldwork, I was somewhat resentful of this discovery I was afraid of becoming saddled with confused findings and thus $I$ did not attach much meaning to it at that time I only recognized its importance later when I suddenly yielded to an impulse to elaborate on the causes and consequences of this divergence in geographical sales orientation between successful central and peripheral firms Furthermore, apart from an emphasis on geographical sales linkages, the research focuses on the pre-existence, the foundation and the early-growth stage of the firms This might have been caused by my foreknowledge of and interest in the subject of new firm formation, gained from previous research experience (Vaessen 1986, Vaessen and Wever 1986) In sum, it cannot be ruled out that other researchers in my place, with other backgrounds and interests, such as labour market issues or subcontractıng, would have come up with different outcomes and conclusions. However, this can hardly be a surprising conclusion, as this problem - the influence of the view of the researcher - is inherent to every kind of research, both qualitative and quantitative

Reliability of the research instrument An indication about the reliability of the research instrument can be denved from a partial overlapping of the three types of questions used, which have been mentioned earlier This technique is sometimes referred to as a form of triangulation, a term introduced by Campbell (see Smaling 1987, 292) In addition to triangulation of interview questions, also member checks have been used This means that results and conclusions are presented to the respondents for verification

To what extent did the nature of the questions posed affect the answers given by the respondents and hence the outcomes of the research? In the present research, the use of both informative questions (facts) and questions about opinions (attitudes) to assess the role of the production environment provides insight into the consistency of the respondents' answers. Thus, the general impression about the importance of the production environment on the basis of factual data is compared with the general picture which emerged from the entrepreneurs' opinions about this matter. Roughly speaking, these two images tend to correspond in that entrepreneurs whose firms maintain abundant linkages with their regional business environment (1 e mainly Randstad firms) tend to attach more value to the influence of the regional production environment for business performance than entrepreneurs whose firms are less firmly interwoven regionally At least, this was suggested by the entrepreneurs' responses to two questions whether they thought they would have been as successful if their firms would have been located in another area of the country, and what they think of the proposition that production conditions are more favourable in the Randstad than 
elsewhere. Besides, this latter question tends to evoke an overreaction among the nonRandstad entrepreneurs. They are inclined to approve every possible location except one in the Randstad. Anyhow, the Randstad entrepreneurs tended to view the locational range of tolerance or spatial margin for production in a more restricted way than the non-Randstad entrepreneurs (Vaessen 1989).

However, this difference in valuation of the local production environment between regionally more and less tightly intertwined firms, respectively Randstad and non-Randstad firms, is ephemeral. It vanishes into thin air when the entrepreneurs reveal what they think the ultimate causes of their success are. Irrespective of their firm's location, the respondents point to either non-spatial external factors (market demand) or to factors internal to the firm, either their own personal characteristics or features of the organization as a whole. Thus, when directly asked to name the ultimate factors of success, locational conditions disappear completely from the picture. This contradicts the impression which emerges from the life histories of the firms on the basis of events placed in a chronological order. These overviews demonstrate that practically every firm investigated, whatever its spatial setting, has derived definite growth stimuli from its local business environment (Vaessen 1989).

In sum, using various types of questions is a useful, albeit rough, method for estimating the extent to which the nature of the inquiries affect the answers given by the respondents.

A second measure for controlling the reliability of the interviews is to hold member checks. This entails sending provisional research reports to all entrepreneurs interviewed. we requested them to verify the content by writing their remarks in the margin. They restricted their comments almost exclusively to the passages about their own firms, and they ignored the (tentative) conclusions. Thus, while insight has been gained about the extent of the entrepreneurs' approval of the empirical accounts of the interviews, this measure did not throw any light on their judgement of the researcher's interpretation of the empirical material. Of course, the entrepreneurs' approval of the research conclusions is a very weak measure of reliability (Swanborn 1990), simply because the researcher's point of reference may be unfamiliar to the respondents.

Reliability of the context Would the research outcomes be the same if the interviews had been conducted under different circumstances or at a different point in time? In response to this question we would like to address two issues which might have been of influence. These are the characteristics of the interviewees and the profitability situation of the firms at the moment the interviews took place.

Eighteen interviews were conducted at 13 firms. It should be noted, however, that the interview scene differs from case to case. The number of respondents participating in an interview, the interviewees' involvement with the foundation of the firm, and the respondents' position and tasks in the firm are combined to form ten different interview situations. During the field work, we conducted:

- five interviews with an entrepreneur who started the business on his own;

- one interview with two directors who founded the firm;

- three interviews with a technical director, one of two head-starters;

- one interview with a one-man starter in the presence of the manager of a foreign subsidiary of the company; 
- one interview with a financial director who is not a head-starter but who is nevertheless narrowly involved in the formation process;

- one interview with a two managers who did not found the business;

- one interview with a general director who was not involved in the foundation of the firm;

- three interviews with a general manager who acted under the supervision of a general director at arm's length;

- one interview with a staff member who is responsible for the firm's public relations and who has been instructed by the general director (founder) after a list with questions had been sent;

- one interview with the son of the founder in a father and son-managed firm.

Undoubtedly, it will influence the answers obtained whether one is seated across two respondents or only one: whether one talks to the general, the commercial or the technical director or whether one interviews the founder himself or (one of his associate(s). On the other hand, however, this is likely to affect mainly expressions of opinions. Information about such matters as the previous work experience of the (other) head-starter, major breaking points or important occurrences in a firm's life history, the geographical extent of input and output linkages and the like seem to be accessible to all respondents interviewed, at least in broad outlines. Of course, it must be noted that the accuracy on some items may vary widely across the interviews.

In addition to the characteristics of the interviewees, a second aspect of the reliability of the interview context is the firms' fortunes at the moment the conversations take place. It should be kept in mind that the first interview round took place when all sample businesses were experiencing high growth rates. Would the answers given have differed if the firms had been in decline? Though the text repeatedly refers to successful firms, it is actually about the good times at the date of the first interview. Thus, the question ought to run: will entrepreneurs give different answers about their relation with the environment and the reasons for their success when they are in trouble and looking back at their hey day? This might be true for their opinions about the causes and sources of the firms' success. However, there is no reason to believe that current prospects would have affected the answers to the informative questions.

In sum, let us conclude this section about the survey's reliability with three remarks. First, qualitative research does not present such neat, exact figures and standards on reliability as quantitative research does. In qualitative research, it is not always possible to present the research process and the research outcomes in a way which makes it possible for other persons to check, to verify and to actually reproduce them. Secondly, this does not imply that qualitative research is completely inadequate and that anything goes. Smaling and Maso $(1990,21)$ propose a more realistic alternative standard for qualitative research, namely debatability and virtual reproducibility. This means that the researcher should give precise information about the methods and procedures used. In this context, Swanborn $(1990,69)$ refers to the criterion of explicitness. This is what we have tried to do in the present section and will attempt to continue in the remainder of this text. Thirdly, the factors which I feel detract from reliability, in the sense of reproducibility, are the idiosyncrasies of the researcher (both interview experience and personal views and interests) and of the 
interview situation.

\section{Validity}

Even if the research were to yield the same results on repeated trials, and the outcomes were perfectly reliable, this does not necessarily mean that they adequately reflect what is going on in reality. Imagine a thermometer with a deviant scale, which consistently and faithfully indicates the wrong temperature. This illustrates the problem of validity of the research findings; i.e., to what extent do the research outcomes adequately reflect real-world processes? However, there is more to it. According to Campbell and Stanley (1963) and in their footsteps Denzin (1978, 199), it is useful to distinguish whether this correspondence between research outcomes and reality focuses on the extent to which the outcomes and conclusions reflect upon the real nature of a process or its representativeness (or both). This distinction is often referred to in the methodological literature as internal versus external validity of research findings (Swanborn 1990). Internal validity refers to the adequacy and accuracy of results. External validity, on the other hand, refers to the problem of generalizing the outcomes.

Internal validity The assessment of internal validity is concerned with the quality of the scientific, causal or logical inferences. Mitchell (1983) defines this as the process of drawing conclusions about the essential linkage between two or more characteristics in terms of some systematic explanatory schemes. The long and the short of it is to eliminate alternative interpretations (Swanborn 1990). In quantitative research, this occurs through reasoning and empirical manipulation (i.e. controlling for or standardizing) in order to avoid spurious correlations. However, no firm criteria exist with which to judge when a relationship between variables has been demonstrated adequately. This value judgement has to be left to the scientific forum, i.e. to colleagues' opinions.

The difficulty with much statistical research is that indicators are rarely specific enough to rule out ambiguity. Adequate explanation derives from an understanding of a process. In this regard, the fact that a relationship is statistically established does not speak for itself. Now, take quantitative explanatory models of regional differences in new firm formation rates. The interpretation of virtually every statistical association observed between a dependent and independent variable can proceed along different lines. For example, according to Storey (1982), home ownership may be directly relevant to new firm formation, because houses can be used as collateral in borrowing to finance new ventures. However, Ashcroft, Love and Malloy (1991) rightly remark that house ownership may also be serving as a general wealth proxy which could influence firm formation from the demand side (through more wealthy local markets) and/or through the supply side, since wealthy individuals would be more likely to have access to the funds required to set up a new firm. Similarly, a managerial background is considered to be important for new firm formation, because managers consider themselves to be more competent to start and run a new firm than bluecollar workers (e.g. Cross 1981; Storey 1982; Ashcroft, Love and Malloy 1991). However, the present research suggests that managers are more likely to start a firm of their own because they maintain the bulk of the firm's contacts with the external environment. Therefore, they are in a much better position to observe market 
opportunities compared with manufacturing personnel (see also Chapter Seven). Many more instances of this lack of specificity in understanding the causes about the concomitant variation of two variables could be mentioned, which must be disconcerting to policy-makers. And then we do not even raise the issue of whether the variables are measured appropriately. To this day, explanatory models in regional science explicate little more than positively established associations between regional differences in firm formation and performance characteristics of the environment. From that observation, these models, of which the centre-periphery approach is the clearest example, proceed to the simplest theoretical solution. And that is that environmental factors determine regional differences in economic performance and structure. A British economist once compared statistics to a bikini: exciting to look at, but the crucial issues are concealed.

Qualitative research is assumed to have greater potential for buttressing the internal validity of its findings. Because of the researcher's extensive direct contacts with the subjects under investigation, he is in a position to lay bare the causal mechanisms of the relation between different characteristics. He is able to do so in greater detail than is possible in a quantitative research design. Two measures are particularly important in this context. These are thick description and the search for counterfactual cases. A third measure, which is of equal importance in quantitative research, is the comparison of the findings with the outcomes of other investigations.

By thick description, we mean a careful justification of the reasoning which links the data with the conclusions. The data gathered are depicted so as to retain the individuality of the answers given by the respondents. This means that the entrepreneurs' responses, including additional motivations, comments and clarifications, will be expressed either in their own words or will be briefly summarized. An important advantage of thick description is that the inevitable interpretation of the respondents' replies by the researcher remains open to external control. This contrasts with the precoded categories in standardized interviews or postal questionnaires, where the transformation of the respondents' disordered 'natural language' into the strongly regulated 'artificial language' of the researcher remains concealed from view. After all, the 'yes' of one entrepreneur need not to be the same as the 'yes' of another.

A second procedure to validate the research outcomes is to take particular notice of the data which do not fit the assumed version of the events. Analysing disconfirming evidence and the search for counterfactual arguments can specify under what conditions a relationship between events may hold and under what conditions it does not. Eyles (1988) emphasizes repeatedly that in the process of validation it is important not to suppress evidence that does not fit. Within this context, the present study will pay particular attention to 'deviant' cases and data.

However, it must be emphasized that internal validity is not sufficient. Presenting a full picture of one or a few cases is not interesting from a theoretical or from a policy point of view until attempts are made to place the cases studied in a wider context. Therefore, the researcher must make sure that he does not lose himself in the individuality of each case. The problem is how to live up to this; it is easier said than done.

External validity The problem at issue under this heading is to what extent the research findings can be generalized across other cases which have not been studied. 
Undoubtedly, this is a strong point in favour of quantitative research methods. In a qualitative case study design, on the other hand, estimating the representativeness of the research outcomes is a precarious activity. Nevertheless, an attempt will be made to design a model of small-firm growth in contrasting regions, against which other cases might be checked. These claims for representativeness of the research findings rest on two pillars. The first is the coherence and logic of the arguments. Mitchell (1983, 198-200) argues that the process of inference to general propositions is always logical rather than statistical. Whether one is conducting quantitative or qualitative research, when analysing the results, one has to go beyond the sample and resort to theoretical thinking to link different characteristics together. According to Mitchell, case studies are just as good a basis for such inference as other kinds of study. The present study attempts to demonstrate that the findings might also apply in other cases by virtue of the logic of the inferences.

A second measure gives some insight into the external validity of the outcomes. This involves comparing the results with the outcomes of other investigations, both confirming and disconfirming external evidence.

Notwithstanding these measures, estimating the representativeness of qualitative research results remains precarious. Therefore, we must stress that generalization cannot be the objective of the present research. We can only present a possible solution to the research question, albeit one directly grounded in empirical observations. Only future research of a quantitative nature will be able to measure the actual representativeness or external validity of the research findings. Nevertheless, a qualitative research design has great potential for identifying and understanding causal mechanisms, due to the researcher's extensive direct contacts with the subjects under investigation. And that is what this thesis seeks, as expressed in the research objective; namely, to develop hypotheses about small-firm growth in contrasting environments by obtaining in-depth knowledge about firm-environment interaction. In other words, a qualitative methodology has been chosen because of its internal validity, not its external validity.

All in all, qualitative and quantitative research complement each other. Qualitative research is more appropriate for theory building and quantitative research is better equipped for theory testing. The methodologies are complementary rather than competitive.

\subsection{The sample}

Which businesses have been selected for the research and how was this selection made? First we will address the procedure used to select the firms investigated. Then We will present the output in the form of a brief description of the firms selected.

\subsubsection{Selection method}

Two main sources were used to track the successful small and medium-sized firms that were investigated within the scope of this PhD project. One is a 'hit parade' of 
the 25 fastest-growing small companies in the Netherlands. The other is a file of firms which once won business awards. The 'hit parade' was published annually between 1986 and 1988 in the periodical 'Intermagazine'. The file of prize winners was compiled by the Department of Geography of the University of Nijmegen (Horvers and Wever 1987, 1989). These sources yielded ten enterprises. Additionally, three firms were traced via the business journal 'Het Financieele Dagblad'. So, in sum 13 firms were selected for in-depth investigation ${ }^{2}$.

The following criteria were used in making the actual selection:

a. The firms had to be at least five years old. (This criterion was applied to make sure that no 'flash in the pan' was included.);

b. The companies should have fewer than 500 employees;

c. There must have been a relative increase in turnover which is above average for the respective industrial category over the four years preceding the (first) interview ${ }^{3}$;

d. During the same period, the firm had to achieve an average net profit as a percentage of total sales (or alternatively of total assets) above the average in the same industrial category ${ }^{4}$;

e. There must have been an annual increase in number of employees over the four years preceding the first interview.

Furthermore, the resulting sample had to include both centrally and peripherally situated firms, so that quite different environments would be represented. Which firms appeared in the footlights after the selection procedure had been applied?

\subsubsection{Overview of the resulting sample}

This subsection gives a general overview of the sample. For a closer look at the firms selected, we refer the reader to the Appendix. Of the firms selected, all but one are high-technology companies. The sample included enterprises engaged in micro-electronics, metal components, synthetics, transport equipment, services related to computer software and hardware, telecommunication consultancy, and rental of temporary business accommodation. Most employees had enjoyed (technical) education at secondary level or higher.

Four of the firms are located in the polycentric metropolis in the western part of the country, known as the Randstad Holland, which constitutes the national economic heartland. The other nine firms are located in the eccentrically situated and more rural northern, eastern and southem parts of the country (Figure 4.1).

With regard to the age of the firms, one can distinguish between nine younger and four older enterprises. Of the younger companies, I was able to interview the founders. The age of these firms, at the date of the first meeting, varied between nine and 19 years. The other four enterprises were all established before 1950 . Since the beginning of the research, ten of the firms have continued to operate independently, while two have been taken over by large multinational corporations and one by a private investment group.

Whereas all 13 firms could be considered fast-growing companies at the beginning of 1988, by the end of 1991 five of them were in serious trouble and 
running at a loss. One even went bankrupt and was out of business in the meantime. In the course of 1992, two more would follow. We will return to these latter firms in Chapter Seven. Without exception, they were engaged in computer-related activities. However, this sectoral bias does not justify any hasty conclusions (cf Section 7.4).

\subsubsection{The quality of the sample}

Before starting the selection procedure, I did not formulate any particular ideas about the sectoral composition of the sample (except that no agrigultural or retail trade firms should be selected), nor were any demands placed on the maximum age of the firms. Looking back, the sample comprises mainly medium and high-technology companies. Within that category, it covers a considerable range of different activities, including both manufacturing and service businesses. Moreover, the sample allowed mutual comparison of high-growth business service firms located in and outside the urbanized core area of the Netherlands. The influence of the quality of the production environment is assumed to emerge most clearly with these kind of activities, while at the same time the relatively wide variation in type of activities reduces the risk of intrusion of sectoral biases in the research findings. Another fortunate feature of the sample is that it contains relatively young firms, i.e. firms of which the founder could be interviewed. This is important, because certain elements of the entrepreneurs' career record preceding the start of their own firm turned out to be important in explaining the growth potential of a number of firms.

However, also in retrospect, the composition of the sample has some shortcomings as well. First, only four Randstad firms have been investigated, whereas nine non-Randstad firms were included. No manufacturing firms in the Randstad were selected. Actually, in order optimally to measure the impact of the regional production environment on small-firm growth, the two groups of firms - Randstad and nonRandstad - should have been matched carefully. This should have occurred on the basis of type of activity, age and ownership. Yet even if I had been aware of this at the beginning of the project, I think that the search for appropriate firms would have been too time-consuming. Let me illustrate this. In order to find 13 firms that were willing to participate, I had to approach about six times as many companies. Those companies refused to cooperate for one of three reasons: they found the research too time-consuming; they considered the topic too confidential; or they did not comply with the selection requirements, on further consideration.

Notwithstanding the shortcomings mentioned, close analysis of the firms selected yields valuable insights about firm-environment interaction in different production environments. On this basis, it was possible to formulate some interesting hypotheses concerning the role of the production milieu for individual business development.

The next chapter, Chapter Five, pays close attention to how the nature of the production milieu affects the companies' environmental or external behaviour. 


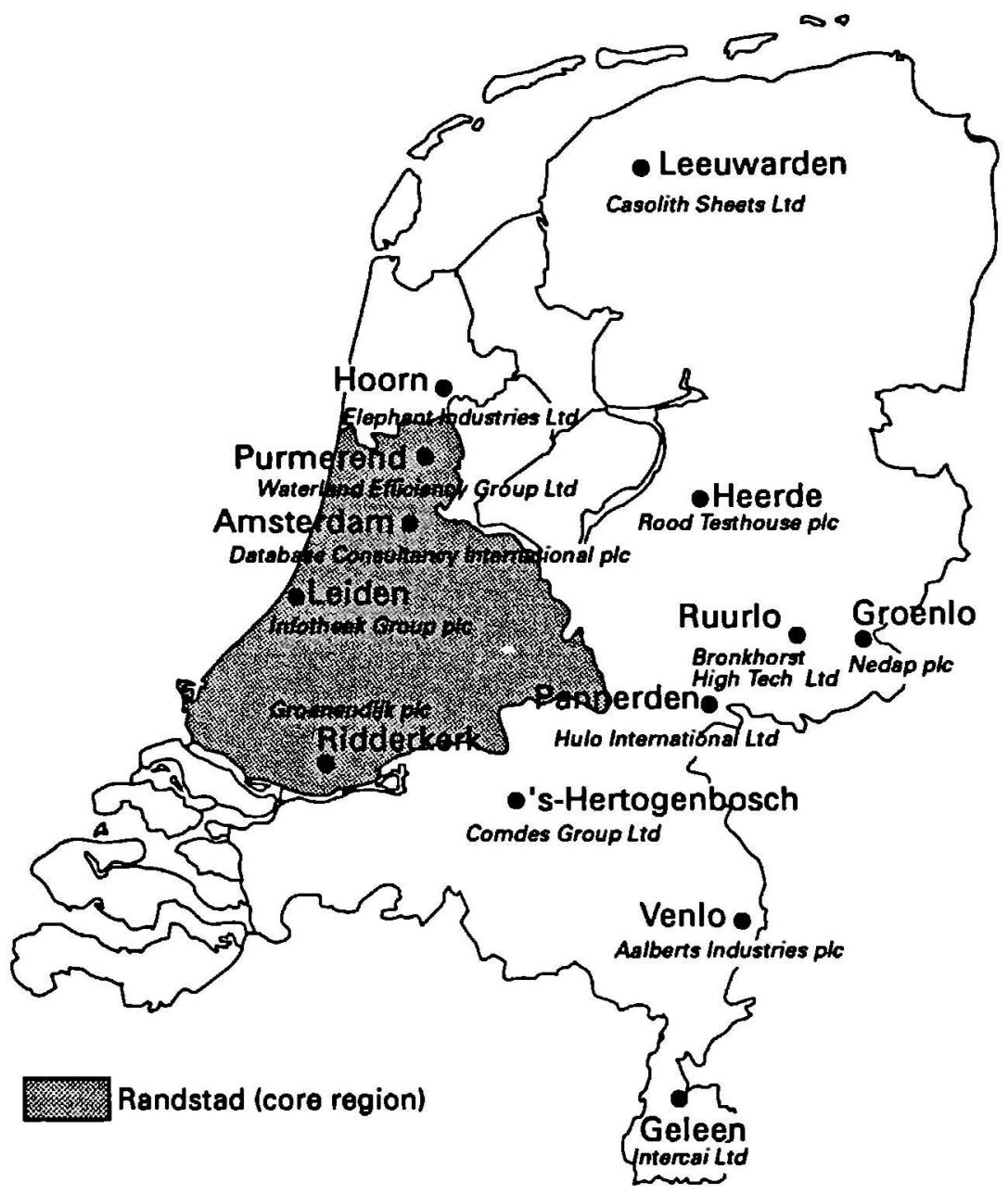

Figure 4.1 The Netherlands; the location of the firms investigated. 
1 In defining the boundaries of the business region, I stick to a circle of about $50 \mathrm{~km}$ around the firm. Some entreprencurs use a slightly different measure for defining the home region, namely the administrative province they are located in.

2 Ideally, it would have been more appropriate to investigate more than 13 businesses; about 25 . However, the processes of selecting appropriate firms, writing letters to and making appointments with directors, and writing out the interviews conducted took so much time that this probably would not have been a viable proposition. Moreover, a large portion of the time available had to be reserved for developing a theoretical and conceptual framework.

3 Sources:

- Annual reports provided individual sales figures;

- ERBO 1984-1987 (VVK) provided average sales figures for the respective industrial categories.

4 Sources:

- Annual reports provide individual net profit figures;

- Statistick financiën ondernerningen (CBS 1990). This CBS publication supplies statistical information about balance sheets, income statements and distribution of income of the major enterprises with economic activities in industry, trade, transport and services. It should be noted that this inquiry excludes firms with a balance sheet total below 10.0 million guilders. Furthermore, for statistical data concerning the year 1991, the CBS periodical Statistisch Bulletin no. 15, 16-4-1992 was used. 


\section{Chapter Five}

\section{Environmental responsiveness of small firms (I): its forms}

\subsection{Introduction}

The aim of the present chapter is to counterbalance the rejection of the individual small firm as a legitimate focus of attention in conventional regional science, including the geography of enterprise. The bulk of economic geographic and regional economic research makes it appear as if small firms can only passively accept the conditions of the environment in which they are located. However, this picture is mainly the by-product of deductively derived and quantitatively tested theories about regional inequality and not the result of positive empirical research. This chapter serves to demonstrate that this perception of small firms is inadequate; at least that it is not a hard and fast rule. It intends to make visible what has been unjustly ignored hitherto in spatial research, namely, direct measuring of small-firm conduct towards environmental conditions, both opportunities and obstacles. More precisely formulated, it attempts to characterize the interaction between small to medium-sized companies and their relevant environments. This formulation includes not only conscious but also unconscious actions which affect the position of the firm in relation to its production environment. This should be kept in mind every time the word strategy is used in this text.

In describing organizational actions, this chapter makes use of the classification scheme developed in Chapter Three. In that chapter, external organizational behaviour was categorized according to its impact on the ties and mutual influences between organization and environment. Four basic 'strategies' have been mentioned, i.e. manipulation, immunization, adaptation, and utilization. It is valuable to point out that this classification was 'developed' (i.e. partly borrowed from Prakken 1987) after the interviews were conducted. So, to be perfectly clear, the interviews were not deliberately designed in advance to find particular classes of organizational responses towards the environment, and certainly not to test the validity of this particular classification. What I endeavoured when I had gathered the empirical material was to grasp and report as adequately as possible from the directors' accounts what was basically going on with respect to the relationship between firm and environment.

The present chapter is organized around the factors of production, i.e. labour, financial means, material supplies, services, and customers. The process of acquisition of these resources will be described and interpreted in terms of the strategies 
mentioned above.

\subsection{Coping with the labour market}

It is widely assumed that local labour markets of metropolitan and large industrial centres are often a source of important economies of agglomeration in production. It is anticipated that the proximity of many firms facilitates the fluid deployment of labour among firms, allowing the firms to meet their labour requirements easily and at low costs from within the local labour market. Angel (1989; 1991), Oakey and Cooper (1989) and others have found indications that firms in dense agglomerations can meet the majority of their labour demands by hiring workers with considerable occupation-specific experience. This pattern holds for professionals and highly skilled technicians as well as for low-skilled production workers. Businesses in remote locations, by contrast, either have to make do with workers at the entry level, generating requisite skills and experience in-house, or they must attract suitable workers from beyond the local labour market (Angel 1991; 1989; Oakey and Cooper 1989). Malecki and Bradbury (1992) note, however, that employers have found it difficult to recruit, transfer and relocate highly skilled and experienced workers to peripheral locations. In sum, such labour market inefficiencies are considered to be significantly detrimental to the competitiveness of high-technology firms, and especially to the competitiveness of small start-up firms (Angel 1991; Oakey 1984; 1985).

On the other hand, Angel notes that the pattern of labour-market activity in dense production complexes, such as Silicon Valley, is not without its disadvantages. The rapid labour turnover in these agglomerations makes producers lose key personnel, who accept job offers from competing firms (Angel 1991, 1509). However, for the majority of (small) producers in Silicon Valley, this loss of personnel to other businesses is apparently counterbalanced by the ability to recruit skilled and experienced workers from the local labour market (ibid, 1511).

Against this background we place the anecdotes of the labour recruitment behaviour and human resource management of the firms in the survey. Since the sample contains centrally as well peripherally located successful young high-technology businesses, the question is, how do these firms deal with the advantages and disadvantages of the local labour market?

\subsubsection{Manipulation}

Investigation of the directors' accounts reveals at once a considerable divergence between reality and the basic image provided by location theory of small firms unable to counteract against their surroundings. This subsection shows the tactics by which the sample firms exert influence on the labour market. These include modifying the external labour supply, enticing the labour force with attractive terms of employment, using intermediate agents as a leverage towards the labour market, and wielding the firm's prestige and reputation. 
Modifying

In 1979, J. Weishaupt and J. Bijsterbosch started their testhouse for electronic circuits in Heerde, i.e. Rood Testhouse. Heerde is a rural nucleus in the far north of the province of Gelderland. Shortly after founding the business the founders were confronted with a lack of 'testing skills' in the labour market. Weishaupt: "Few people could be found who had prior work experience with 'testing'. This means that education had to take place on the job. We established relations with the HTS (Technical College) in Zwolle [20 km] and we began to attract students on work placements. We broke these people in while doing their work placements and we could take advantage of the opportunity to select the most eligible ones. At present obtaining suitable workers is not much of a problem anymore." In this way Bijsterbosch and Weishaupt to some extent created the requisite labour supply which they could not find.

Nedap (detection systems) takes a similar route. Although director Westendorp is not dissatisfied with local labour supply, he made some critical comments regarding the degree of motivation. This criticism was aimed at the educational institutes from which Nedap recruits new personnel. "I would like the teachers to make the students more eagerly. We make extensive use of students on work placements, on average 30 students a year. While they are doing their teaching practice here, we try to make them enthusiastic for working in our firm, and meanwhile we select the best ones." Also Bronkhorst High Tech (gas flowmeters) regularly recruits new employees from students on work placements, both on a secondary and a tertiary level of education. Engaging students on work placements, breaking them in in the firm, and trying to recruit them when they finish their study and enter the labour market is an example of how small firms may organize (local) labour supply.

A related method of how firms may modify (selected) segments of the local labour force before actually trying to enlist particular workers is displayed by several businesses in the sample which are engaged in information technology. These are System House Comdes, Waterland Automation (both computer software engineering), Intercai (telematics consultancy), and Infotheek (computer trade). As System House Comdes was growing rapidly in the early and mid-1980s, the founders Quekel and Kragtwijk were increasingly confronted with a shortage of automation experts within the labour market. At one point they decided to hire unemployed academics and people who had enjoyed higher vocational education. By means of additional inservice training, they then tried to impart the requisite automation skills to the newly hired workers. As a by-product the company gained know-how about training and schooling of personnel. When sufficient experience had accumulated, the Comdes management decided to commercialize this know-how by providing educational services in the field of computer sciences to other organizations. One of Comdes's clients is the Local Employment Exchange (GAB). Now, a situation has developed whereby the company takes highly educated though unemployed people and makes them suitable for work in its own kind of business at the expense of the GAB. A great advantage to Comdes is that the firm has first choice and ample opportunity in selecting the most appropriate students and offering them a job. Comdes is able to hire a substantial number of new workers via its Elementary Course in Automation (BOA).

Similarly, Waterland Automation, in its heyday, made use of unemployed, highly educated people who were registered with the local GAB. In those days, a shortage of 
eligible employees was the most significant factor curbing the growth of the firm. Schoneveld, in 1988: "If you ask me what dampens our growth, then the answer is shortage of suitable manpower." According to Schoneveld, the firm had to refuse jobs offered because it lacked sufficient manpower. Cooperation with the local Employment Exchange was one of several endeavours to tackle this problem. Schoneveld: "In cooperation with the Local Employment Exchange we retrain people who have enjoyed higher education in directions which offer no future prospects for employment. These persons have to be trained from scratch. We used to start with a group of 15 persons. It is the aim of the project that Waterland will engage one-third of those who complete the course. Actually, we employ all persons who have finished the course; about one-third drop out during the training. By the way, we are talking about lower-order functions of the firm, the programmers. We have been doing this now for three years and I am satisfied with the project."

In 1986 director Kivits of Intercai was requested by the GAB for Maastricht and its surrounding area to provide a one-year telematics consultancy course for unemployed academics and people with a higher vocational training. While the Local Employment Exchange financed the course, Intercai took care of the subject matter and the organization of the training. According to Donders, by 1990 Intercai itself already attracted about ten people from the course out of the 100 persons who had taken it.

A final example of modifying skills on the local labour market is provided by Infotheek. In 1989 the company together with four other leading Dutch automation companies announced the foundation of a new sectoral interest group which will be aimed at monitoring the quality of automation courses and trainings in the Netherlands. This initiative has been taken in order to combat the morbid growth and bungling in the field of automation education and their adverse influence on the quality of the labour market (Het Financieele Dagblad, 25-1-1989).

\section{Terms of employment}

The businesses studied do not only affect the labour supply through providing education and training courses for the external labour force. They also exert their influence more directly by offering attractive conditions of employment to potential workers, either financial or fringe benefits or both. When Intercai reached a size of about 50 employees, director Kivits wanted to strengthen the managing staff with a second director. He succeeded in attracting Mr. H. Waalboer - "the best one can find in the Netherlands in the field of telecommunication consultancy", according to Kivits - away from Alcatel-Holland. He attracted him not by paying premium wages but merely by offering better fringe benefits. Kivits: "At Intercai, Waalboer earns exactly the same salary as he did at Alcatel. With us he is no better off, not even a single guilder. What does count is that he has acquired more freedom and responsibility by joining Intercai. And that is what we intend to give our employees in general, freedom and responsibility. Furthermore, they have the opportunity to buy Intercai shares, and we offer other benefits in order to make employees feel comfortable."

Director Vissers of System House Comdes also pointed out the role of fringe benefits in obtaining access to the external labour market. In response to complaints by director Vissers about the constrained capacity of the regional labour market, I asked him whether this situation is harmful to the performance of System House Comdes. Vissers: "This is only a modest problem for us since we are situated on the right 
side of 's-Hertogenbosch, with good road connections with the Randstad, and on the other hand we work out of a magnificent office building with many conveniences. People can do their job here very comfortably; parking is no problem, we have ample parking space, and our office is very accessible."

By the way, offering good fringe benefits in itself may not be adequate to attract the entire staff, and additional means may prove necessary. For example, though Intercai did succeed in hiring Waalboer because of favourable fringe benefits, this does not mean that other top personnel has been captured in this way too. The remark of staff member Donders that "as the company grows, it can catch the bigger fish" is indicative of a different way to induce professionals to move from their previous employer to Intercai, i.e. the wage lever. This occurred in the case of Mr. F.M. van der Voort; this third holding director, who is responsible for the internationalization of the firm, was drawn away from Enicsson-Holland (located in Rijen, at a distance of $129 \mathrm{~km}$ from Geleen). Another instance was the case of Mr. C.H. Kouwenhoven, who is responsible for the development of the Dutch subsidiaries commercially, and also for public relations and advertising domestically as well as internationally. Kouwenhoven was previously director of a software company in Rotterdam (Het Financieele Dagblad 8-8-1992), which is at a distance of about $180 \mathrm{~km}$ from Geleen'.

The financial 'crowbar' has also been wielded by director Vissers of the Comdes Group. Under the regime of Quekel and Kragtwijk, Comdes hired its workers mainly (about 90 percent) from the local business region, i.e. the province of Brabant ${ }^{2}$. In 1989 Comdes was taken over by the investment group Modalfa, and a new managing director, Mr. Vissers, came to the helm. At that point, the course changed rather drastically. Vissers was not impressed by the abilities and skills of a number of staff members of the Old Guard. Through a major reorganization, he wanted to raise the quality of the firm's services and lift the company's functioning to a more professional level. Director Vissers: "I have tried to remove the weak core of employees. I replaced the entire management of the [six] business units, except one, and 75 per cent of the middle management [project managers] by hiring new people from outside." In concrete terms, Vissers is talking about ten executives who have been supplanted. Apart from that the new Comdes director acquired 44 new employees for lower positions (after reducing the original number of employees from about 160 to 116). To acquire new management staff, Vissers called in headhunter agencies. Vissers: "I gave them a profile of which type of workers I wanted and I told them also which companies I would prefer the new managers to come from." It stands to reason that to achieve this, Vissers had to manipulate the labour market by offering additional financial inducements. These were amply supplied by the new owner, i.e. the substantial investment group Modalfa/Mobeta Ltd. Applicants for lower positions were enlisted via advertisements.

\section{Leverage}

Yet another strategy in operating on the local labour market to acquire suitable staff is by using intermediate agents as a lever for gaining better access to the labour market. Hulo Intemational (transport equipment), for example, maintains good relations with relevant local training and education institutes. Director Meeus: "If one has good connections with the director of the Technical College in Arnhem [15 km], or with the director of the Intermedinte Technical School in Doetinchem $[25 \mathrm{~km}]$, it is always possible to 
get, on request, some suitable people [i.e. students] in one's office for an interview." One step further is the incorporation of people from the field of education into the Supervisory Board or even into the Executive Board of Directors, as is the case with parttime professors, for example. They might be motivated to bend and influence the stream of graduates in the direction of the enterprise. Infotheek, Rood Testhouse and Elephant Industries have professors in their Supervisory Board. Director Brouwers of Elephant Industries indicates that he has acquired scientists via this channel. Brouwers: "Graduates from universities have joined our company because of such contacts with universities." These examples illustrate that organizations need not resign themselves passively to local labour force constraints. They may play an active role within the labour market and try to influence its disposition in favour of the firm's requirements.

\section{Prestige and reputation}

Apart from purposeful actions, such as supplying external training or providing tempting employment conditions, an organization can influence the external labour force in its favour in yet another way. This alternative may be less direct or purposive, but not necessarily less effective. I am referring to the way an organization wields its prestige. This point arises most clearly in the interview with director $\mathrm{K}$. Groenendijk of Groenendijk plc (letting of makeshift accommodation). When asked whether his business experiences difficulty in attracting new employees, Groenendijk's answer was: "I know that there are complaints in our line of business about this matter. However, it hardly gives us any problems. It is snid that there is a shortage of drivers, but we do not notice it in our firm... the reason for this is the business's reputation."

Likewise director Meeus of Hulo International (transport equipment) brought up the point of the firm's image in acquiring new employees. It appears that Hulo International is regularly approached by people who would like to join the company as an employee. Meeus: "They simply find it fascinating to work for us." A major reason for this attraction of the firm to the (local) labour force is, according to Meeus, the captivating product it makes. Meeus: "one can choose to be engnged in the production of dull hose-pipes for vacuum cleaners or of more risky [challenging] machines." Hulo's transport and logistic systems, special handling equipment, robot manipulators and related appliances are custom-built and Meeus regularly calls them 'solutions' to customers' logistic problems.

Director Van Leenen brought the image of his firm, Infotheek, to the fore in response to a question concerning labour turnover. Van Leenen: "Sometimes staff members are bought away from us, though at present less frequently than formerly. I think that the prominence of our company due to its quotation on the Stock Exchange gives us greater possibilities to retain our persomnel." Also Schoneveld and Haakmeester (both of Waterland Automation) noted that people find it interesting to work for Waterland, although they do not always possess the right qualities.

The role of prestige is also underscored in the literature. Thompson (1967) states that: "Acquiring prestige is the 'cheapest' way of acquiring power. To the extent that an environmental element finds it prestigeful to exchange with an organization, the organization has gained a measure of power over that element without making any commitments; i.e. it has gained power without yielding power" (Thompson 1967, 33). 
He refers to Perrow (1961) who concludes that if an organization and its products are well regarded, it may more easily attract personnel, influence relevant legislation, wield informal power in the community, and ensure adequate numbers of clients, customers, donors, or investors. Perrow sees the creation and maintenance of a "favourable image of the [hospital] organization in its salient publics" as an important way of controlling dependency (see Thompson 1967, 33).

The foregoing shows (some of) the tools that small and medium-sized enterprises may have at their disposal to influence the disposition of the external labour market in favour of the organization. At the same time it suggests that one has to be cautious when interpreting complaints by directors of small and medium-sized enterprises about labour shortages. At first sight these might be recorded and understood merely as constraints of the production environment. However, upon closer scrutiny, these complaints may reveal important organizational weaknesses as well. Rather than real external inadequacies they might reflect the firms' weak competitive position within the labour market and their inability to generate sufficient applicants on their own. And it is this internal dimension which tends to be overlooked in the quantitative research tradition of regional science.

\subsubsection{Immunization}

Although the businesses investigated demonstrated some ability to manipulate (sections of) the external labour market to benefit their own requirements, this often proved to be insufficient. In the first place, it may not be practicable to influence all workers before they enter the firm. It may be too expensive, for example, to lure away from competitors or other enterprises every single worker needed who is not ready available in the labour market by dangling the purse in front of him. In the second place, even if an entrepreneur has succeeded in inducing the (type of) workers which, given the situation in the labour market and the financial capacity of the firm, he had in mind to join the firm, he is not there yet. After all, these new employees may not all be brought into action at once. Consequently, entrepreneurs, if they want to retain or improve their current market position, often have to take additional measures which make their firms relatively insensitive to inadequacies or deficiencies of the external labour supply. Two forms of immunization could be observed from the interviews: namely, what has been labelled (internal) integration and rationing in Chapter Three.

\section{Integration}

One measure is to compensate for a certain lack of skills or know-how by internal education and training. And indeed, from the interviews it becomes abundantly clear that the firms studied are or were heavily engaged in training and education of new and existing personnel, although not every firm gives exact figures on investments in human resources. Director Vissers of System House Comdes assessed that the costs of training programmes would amount to somewhere between 3000 and 4000 guilders per employee annually from 1992 onwards. This is 2100 to 3100 guilders in excess of average costs of training programme in medium-sized commercial businesses in 1990 
in the Netherlands (CBS 1992, Table 2). For programmers, this training consists of technical courses in new programming techniques, whereas sales staff take courses on new sales methods. For the management staff, structural schooling expenditures are even higher, from 8000 to 10,000 guilders per manager. This is mainly because Vissers includes the expenditures for visiting symposia and seminars among training costs. The bulk of training costs consist of indirect costs, i.e. the costs of lost working hours. For internal company training programmes, Comdes uses its own training unit, which also operates on the commercial market. Direct training costs, on the other hand, consist of payments for courses which Comdes contracts out to external agents. Vissers mainly farms out external training programmes to computer firms like IBM and DEC, or to software firms like Progress and Uniface. By the way, in 1990 and 1991 schooling costs for the Comdes Group were even higher. Vissers estimated that he was going to spend about 1.2 million guilders per year in this two-year period. Per employee this would mean education expenditures of about 6000 to 8000 guilders, according to Vissers. These huge expenditures for education in the years 1990 and 1991 were mainly because of a major reorganization the firm went through in this period. Vissers: "In 1989 we decided to drop developing software for Wang computers, which was until then our sole hardware base. This means that we have to retrain our personnel to be capable of working in the IBM/AS 400 environment, in the DEC environment and in a Unix environment. This training takes place partly here at our office and partly at the sites of the hardware firms."

Similarly, under the regime of Vissers's predecessors, Quekel and Kragtwijk, who founded the Comdes Group, the firm spent much money on training their human resources, not only on teaching programming techniques and principles, but also on developing the communication skills of the workers. Kragtwijk: "We are particularly in need of employees who are communicative and who are good at dealing with people. For that we have developed separate courses." Kragtwijk and Quekel did train their workers in holding conversations, in chairing meetings, in giving lectures and so on. Kragtwijk: "Doing business is a matter of confidence, which one must be able to radinte".

Also director Schoneveld of Waterland Automation declares that inadequate know-how of programming techniques is not the main deficiency of new personnel. In the first interview - April 1988 - Schoneveld said: "The most important problem we have is education. I mean the whole course which is needed to clarify the function a software package fulfils in a business company, and how people in client firms make use of it. One has to realize that every new staff member costs us 80,000 to 100,000 guilders before he is fruitful. I find that the subject of administrative science and how to apply it in practice should become obligatory in a whole range of fields of study." Schoneveld was convinced that such a measure would considerably diminish Waterland's expenditures on additional on-the job training of new personnel, and would make them more useful, more quickly. Furthermore, Schoneveld found that new employees' knowledge of automation is not up-to-date.

In addition to these structural education costs, Waterland, just like Comdes, occasionally has even still higher training costs. In 1985 director Schoneveld started a reorganization of Waterland Automation. Schoneveld intended to evolve greater specialization in developing software packages for computerized control of logistic processes in manufacturing production. This is supposed to improve, according to Schoneveld, "Waterland's visibility in the market". By 1988, when Waterland Automa- 
tion was suffering losses, the specialization strategy was intensified. The core business, i.e. developing software packages in the field of logistics automation, was strengthened and extended vertically, both upstream (i.e. automation consultancy) and downstream (after-sales services). Another reorganization measure was to achieve better standardization of workers' programming practices. To implement these and other measures, the whole organization had to be retrained, partially in-house and partially elsewere. In 1988 and in 1989, employees of Waterland Automation spent on average no less then 10 per cent of their working hours on education. Schoneveld explicitly wanted to carry out the reorganization through retraining existing workers instead of acquiring new personnel. The organization's management even received additional schooling in how to initiate and guide the reorganization process. Schoneveld: "Our policy is to realize the entire reorganization with our own employees. Of course in a number of cases this will be more expensive than attracting new people from outside who have more approprinte know-how. However, knowledge is not the whole point, settling into an organization is an equally important aspect. It requires in some cases much time before new people are productive. This also applies to workers with appropriate expertise; they come from a different organization characterized by a divergent business culture. Before they have adopted our culture, valuable time is lost." This decision was also inspired by bad experiences with acquiring new personnel in the past. Existing staff members felt their position in the firm was threatened and decided to leave. Schoneveld: "For a type of business like ours, it is very important that labour turnover is as low as possible" (see box).

Intercai (telematics consultancy) also has to supplement the skills of newly acquired and existing personnel with additional internal education in order to avoid becoming negatively affected by conditions on the labour market. Though the firm mainly requires highly experienced consultants (Director Kivits: "Shell or the Amsterdam Stock Exchange do not pay us to be advised by people who have just gradunted. These organizations require top consultants, the best they can get in this field."), it is not able to select exclusively this kind of workers. Intercai also hires junior staff at the entry level. Kivits: "Otherwise salaries are prohibitive." Furthermore, Kivits says: "I could use 20 or 30 workers who are specialized in some subsectors of telematics consultancy, but unfortunately they cannot be found in the Netherlands. So we have to largely create them by ourselves, through giving them the opportunity to build up expertise". Fellow worker Donders estimates that two-thirds of all new personnel has already gained substantial experience in the field of consultancy during their previous work experience. Inexperienced, newly appointed junior employees are exempted from any productive task during the first two months on their new job. However, it takes two years before they have become full consultants. In the 1988 interview, Kivits indicated that in-house education consists "partially of on-the-job training, and partially of courses which we give them ourselves." To shorten the breaking in period of new personnel, Intercai started a new experiment in the field of education of human resources in 1989. Some six newly appointed, highly educated employees at the entry stage were offered the opportunity to take a six-month, full-time training programme. This course was developed by the education unit of Intercai. Donders has the impression that the learning process is more efficient in a structured education programme than by means of training and supervision on the job. While he is not able to present evidence, Donders feels that employees who have followed the full-time training course can more easily tackle 
These observations of director Schoneveld indicate that David Angel in his previously mentioned article on High-technology agglomeration and the local labour market did not fully cover the disadvantages of rapid labour turnover. Angel refers only to the damaging effect of the loss of expertise of frequent interfirm mobility of personnel, which, by the way, would be counterbalanced by the easy influx of skilled and experienced workers in dense production complexes (Angel 1991, 1511). Director Schoneveld, however, adds to this other harmful influences of intensive labour turnover. He mentions the costly break-in period of new personnel, which is all the more difficult because these workers have often previously worked in a different corporate culture. According to director Westendorp of Nedap (electronic detection systems), Westendorp: "changing people and unlearning them things is more difficult for us than teaching them sonething new."

Furthermore, Schoneveld points to the negative and threatening effects newly hired, highly qualified employees may excrt on existing employees, especially when the former occupy higher-order functions, which the latter feel passed over for. Schoneveld also complains about the loss of customers that occurs because of high labour turnover. Employees who leave the firm to start a business of their own often tend to take customers with them. The case of Casolith Sheets (acrylics products) shows that customers not only stick to employees who leave their supplier (of computer software) to start a new business, but also to employees who only move from one employer to another.

And it is director Jouwsma of Bronkhorst High Tech (electronic flowmeters) who mentions yet another destructive effect of rapid labour turnover, namely, poor and disruptive customer service. Bronkhorst High Tech uses IBMcomputer hardware. For software development, IBM refers clients to a company in Diemen, which lies a stonc's throw from Amsterdam. While the Diemen company should have completed its task for Bronkhorst by July 1987 , at the dates interviews took place, i.e. early 1988 and early 1990, this had still not occurred. An important reason for this delay is the disruption of the software services related to Bronkhorst, because the executive computer scientists did not stick to the Diemen company. In the 1988 conversation, Jouwsma said: "Now we are dealing with the third executive of that firm. We have to keep explaining over and over exactly what we want them to do." Two ycars later, the project was still not ready; again, another computer scientist was engaged with it. According to Jouwsma it took three months before the new executive picked up the thread where his predecessor was. Jouwsma was furious at the Diemen company: "It is little less than scandalous .... It is a menace, I can tell you that." If other clients of the firm in Diemen have complaints similar to those of Bronkhorst, the damaging effect of rapid labour turnover may be fatal to the automation company.

A final disadvantage of high labour to be mentioned here, is that it adversely affects companies' training and education efforts. Reich $(1989,24)$ writes: "The restlessness of U.S. workers creates centres of technology like Route 128 around Boston and Silicon Valley in California, but it intends to discourage investment in human capital."

On the basis of these and other examples, one may wonder if Angel is underestimating the disadvantages of rapid labour turnover in dense production complexes when he states that the loss of personnel to other businesses is apparently counterbalanced by the agglomeration advantage of easy influx of new skilled and experienced workers from the local labour market. 
tasks by themselves.

So it appears that by means of in-service training, Intercai seeks to secure the quality of its work in spite of some shortcomings of the external labour supply. For the recruitment of the eight division managers that Intercai needed, the firm relied on the external labour market for only three. The other five division managers were recruited from its own ranks. Intercai has established internal job ladders, up which selected workers can advance and gain management experience.

Bronkhorst High Tech, manufacturer of electronic flowmeters, provides another telling example of how internal education prevents businesses from becoming adversely affected by deficiencies of external labour supply. Director Jouwsma: "Newly graduated electronic engineers cannot be used immediafely, because, for example, they don't know anything about flowmeters. Or if I would ask them to make a 'print', they would not know how to do it. They are simply not practically educated. We actually have to educate them ourselves. After six months' in-service training they are able to work in our firm." When asked whether this situation dampens the growth of the firm, Jouwsma answered: "In the beginning it did, becnuse while one staff worker was tenching the other, together they produced too little output. Yes, at the outset this situntion did cause a problem. When the firm became bigger, however, we could afford a separate education official. Now, it does not cause problems anymore." Furthermore, Jouwsma indicated that apart from the education official, he himself teaches courses to employees, every Thursday afternoon. By the way, the education official was recruited internally. Jouwsma: "He is the very first employee we engaged. He knows everything, and he enjoys working here."

Similarly, the other firms investigated are or were heavily engaged in internal education of human resources. Casolith Sheets (acrylics products), for example, spends about 100,000 guilders per year on training its staff. This amounts to about 690 guilders per employee. For medium-sized firms (size class 100 to 500 employees) in manufacturing industry as a whole, the average equivalent figure would be only about 350 guilders (calculated on the basis of figures from CBS 1992). And director Spoelstra even excludes an amount of 300,000 guilders, which is invested in quality circles, because this is an incidental expenditure. Nedap (electronic detection systems) spends two per cent of direct labour costs on education and training of employees (Nedap 1988, 23). For industrial firms within the size class of 100 to 500 employees, this figure amounts to a mere 0.8 per cent (CBS 1992, Table 3). At Bronkhorst High Tech in 1990, 32 staffmembers followed an external course in addition to the extensive internal training programme. These 32 employees represent at least 0.7 participants in (external) training programmes per employee. This figure in itself is far in excess of a mere 0.09 participants in (internal and external) training programmes per employee in small manufacturing firms (size class five to 100 employees) as a whole (Statistisch Bulletin 13-2-1992, 6). Just for the record, training efforts of firms cannot always be interpreted as compensation for deficiencies of the external labour force. Director Groenendijk of Groenendijk plc (transport and letting of makeshift accommodation), as well as director Brouwers of Elephant Industries (precious metal dental products), also have to provide additional in-service training; nevertheless, they are content with the local external labour supply and acquisition of new employees. Director Aalberts of Aalberts Industries (supplier of tailor-made metal products) says: "We prefer new Junior Technical School graduates at C-level, who are prepared to undergo additional in-house training." According to director Westendorp of Nedap, it is impossible for an educa- 
tional institute to turn out ready-made workers, because every firm has its own peculiarities, which it has to impart to employees by itself. Westendorp even takes the argument a step further: "I would not advise educational colleges to try to do so. Probably they would do things a little different from our own method. Well, changing people and unlearning them things is more difficult for us than teaching them something new." So, for several reasons and within certain limits, firms might prefer training staff in-house to hiring highly experienced workers from outside, irrespective of their availability on the labour market (see box).

Anyway, the firms studied do not resign themselves to mismatches between qualities offered by the labour force on the one hand and requirements of the business on the other hand. As such, in-service education and on-the-job training are tactics which can be applied to compensate for inadequacies of the external labour market.

\section{Rationing}

From a strictly spatial or regional point of view, firms can make themselves immune to inadequacies or shortcomings of the local labour market in another way. They can, to a certain extent, retreat from or bypass the local labour market and try to get access to extra-regional labour markets. From the experiences of the businesses examined, two methods can be inferred. One is that attempts are made to attract people from outside the local business area to the firm. Director Vissers (System House Comdes) provides the clearest example of this tactic. When he cannot find sufficiently professional computer programmers and managers within the local business environment, which is 's-Hertogenbosch and its surrounding area (50 km radius), he succeeds in attracting them from the Randstad. Vissers: "I have tried to find them in the local environment, within a radius of $50 \mathrm{~km}$. I did not succeed. Ultimately I think 30 per cent of the new personnel comes from within this radius of $50 \mathrm{~km}$ and 70 per cent from outside this circle." This holds for both higher and lower-level personnel. Vissers: "The problem with the present local business region is that too few professional, highly qualified software system houses are located in this area. There are four or five good enterprises in Eindhoven [32 km], but that's the end of it." Most new employees appear to come from cities in the Randstad: Utrecht $(55 \mathrm{~km})$, Rotterdam $(80 \mathrm{~km})$ and other places.

Staff worker Donders observes that the local response to recent employment advertisements of Intercai Geleen in national papers has diminished. Nonetheless, this does not affect the firm, since this trend has been balanced by a growing response from outside the province of Limburg. From the two interviews conducted at Intercai Geleen, it can be inferred that the number of workers who come from within the province of Limburg decreased between 1988 and 1990 from about 85 per cent to about 60 per cent. And though this is not a conscious strategy, its effect remains the same; namely, reducing the firm's vulnerability to the situation within the local labour market.

Nearly the entire top and middle management of Elephant Industries, about 20 persons, come from places within the Randstad ( $35 \mathrm{~km}$ distance and over). They have been acquired in several ways. Some have been found by directly phoning educational institutes to request suitable applicants; some have been acquired via advertisements; some academics approached Elephant Industries via existing contacts between their university and Brouwer's firm; others asked director Brouwers for a slot in his 
firm after they had attended a lecture by Elphant Industries. Recently the firm called in an acquisition and selection bureau in Rotterdam $(100 \mathrm{~km})$ to track down suitable staff members for Elephant Industries. Furthermore, Brouwers says: "But manty of those people coming from Amsterdam, Zaanstad, and the Randstad, after a while, decide they are going to live here too, and then they move to Hoorn."

As an alternative to bringing distant workers to the firm, extra-regional labour markets may be addressed by bringing the firm to the workers. This is a strategy frequently described in articles on the location of high-tech activities (see for example Keeble 1986; 1988; Perry, Dean and Brown 1986). Complete relocation of a firm for reasons of labour market considerations has not been observed among the sample firms. However, a related method has, which will be dealt with in the following.

\section{Scattering}

Intercai and Database Consultancy Intemational happen to have adopted a locational pattern of small plants scattered throughout the southern and western part of the country. In 1990, Intercai comprised eight subsidiaries, spread across five different provinces. In 1988, Database Consultancy International consisted of four establishments located in three different provinces, all of them within the western part of the Netherlands. This dispersal enables these firms to tap the labour markets of almost the whole western and southern parts of the country. Although this was not the main reason behind the strategy - it was mainly pursued to avoid becoming large bureaucratic organizations - this pattern offers important advantages within the labour market. As a result, the firms have become far less susceptible to labour force constraints within their original local region. Intercai again provides a clear example of this. In 1989 the firm established a specific subsidiary which collects specialist knowledge. This subsidiary, 'Special Know-How', employs six researchers who are highly experienced in the field of computer science and telecommunications. Special Know-How is accommodated in the Intercai office in the city of Utrecht, which is part of the Randstad and at the centre of the country. Since all researchers at 'Special Know-How' come from organizations sited in or near the Randstad (Siemens, Alcatel, PTT, Ericsson, universities), one might doubt whether the company would also have succeeded in recruiting them if they all had to move to the head office in Geleen. And it is out of the question that similar expertise could also have been found in the province of Limburg. So, by applying a multilocation strategy, firms scatter their labour market relations across space and thereby lessen dependency on the conditions in one particular area.

\subsubsection{Adaptation}

The sample provides few examples of active adaptation (McDermott and Taylor 1982) to the labour market, through which firms do not try to modify the dimensions of the labour force or seek to hedge against it, but, instead, constructively adjust to them. However, without any doubt active adaptation will have occurred. After all, for most of the 1980s, the labour market was characterized by shortages of skilled and experienced technicians and computer scientists. Furthermore, there is an ever-present 
shortage of professional managers. These types of workers are in a position to place high demands on potential employers. At least some of the sample firms must have been confronted with this and will have adapted to the demands and desires of these workers, either in the sphere of payments or in the sphere of fringe benefits. This subject, however, was not raised during the conversations with the directors.

On the other hand, a case emerged of a more passive form of adaptation, whereby a firm's products were adapted to the type of skills and expertise available in the labour market. In 1985, Schoneveld decided to narrow down Waterland Automation's activities and specialize in automation of intra-firm logistics. When asked which activities the firm pursued in the years before, Schoneveld gave the following answer: "During the years before, we set about automation projects which were determined by the know-how and prior work experience of the staff workers who were in our service at that moment rather than by the aims and intentions of the company, a situation which one could find in most system houses." And then Schoneveld continued: "Now, we more empitatically search for new employees who have a background in manufacturing automation."

\subsubsection{Utilization}

When all is said about the companies' abilities and endeavours to deal with discrepancies between firmneeds and the local labour market, it remains true that all businesses in the sample, both Randstad and non-Randstad firms, are able to take advantage of the local labour force as well. This is most clearly demonstrated by four industrial companies and one service company from the sample, which are located in the eastern and northern periphery of the Netherlands. These firms are Casolith Sheets (synthetics), Bronkhorst High Tech (electronic flowmeters), Nedap (electronic detection systems), Hulo International (robotized transport equipment) and Rood Testhouse (testing electronic circuits). All five firms obtained over 90 per cent of their employees from the local business region, and none of them was particularly dissatisfied with the regional labour supply (this does not mean, however, that no mismatches between firm and labour market occurred). The education infrastructure in the Dutch periphery may be largely sufficient for a high-tech company, as shown by Bronkhorst High Tech (BHT). Bronkorst High Tech is located in Ruurlo, a rural village of less than 4000 inhabitants. Ruurlo lies in the centre of a peripheral region on the eastern border of the Netherlands, a region known as the Achterhoek. The main education institutes from which the employees of Bronkhorst High Tech graduated are located at a distance of $35 \mathrm{~km}$ to the northeast (Intermediate Technical School and Technical College in Enschede), $33 \mathrm{~km}$ to the northwest (Intermediate Technical School in Deventer), $20 \mathrm{~km}$ to the south (Intermediate Technical School in Doetinchem), and $40 \mathrm{~km}$ to the southwest (Technical College in Arnhem). Director Jouwsma perceives Bronkhorst as being centrally located at the intersections of the market areas of these institutions. The employees themselves all live within a radius of $30 \mathrm{~km}$ from the firm.

Some $13 \mathrm{~km}$ eastward from Ruurlo, via a scenic route, lies another rural nucleus in the Achterhoek, called Groenlo (8186 inhabitants). This is the location of Nedap, another high-tech firm in the sample. Both directors of Nedap whom I interviewed, 
Mr. Paijens and Mr. Westendorp, are content with the local labour supply. No less than 99 per cent of the firm's employees come from within a radius of $40 \mathrm{~km}$. Nedap makes use of largely the same educational institutes as Bronkhorst does, though it takes on more scientists. Paijens: "Most of them have studied at the Technical University of Twente [in Enschede, 37 km]."

Continuing through the rural landscape of the Achterhoek, travelling from Groenlo in the direction of Nijmegen over back roads, after some $53 \mathrm{~km}$ one might pass through a small village of about 2000 inhabitants. This is Pannerden, home base of Hulo Intemational a third high-tech company in the sample. Although this firm got into trouble in 1990, it was successful at the time the interviews took place. Anyway, in early 1988 I was on my way to visit Hulo International, and I eventually found the firm on the outskirts of the village, in the open fields. The lowest level of education which Hulo requires of its employees is intermediate administrative or technical education. "Otherwise", according to director Meeus, "one would just not be able to fulfil one's task in our firm." Furthermore, about 30 per cent of Hulo's employees have a higher vocational or scientific education. Out of the 85 people which Hulo employed at the date of the interview, only five (six per cent) came from outside of the local business area $(\geq 20 \mathrm{~km}$ ). These are highly skilled workers. Director Meeus was very content with the skills and the work ethic of the regional labour force. Meeus: "Skilled workmanship is very good in this area, and also loyalty to the company and willingness to work overtime." However, he severely criticized education institutions for going overboard in teaching abstract theoretical knowledge, which is virtually useless in practice. Meeus blamed educational institutes for the fact that: "It takes an incubation period of at least five years before an employee who has just finished a Technical College is able to master his tasks in our firm completely." Two educational institutes from which Hulo International obtains its employees are the Intermediate Technical School in Doetinchem $(22 \mathrm{~km})$ and the Technical College in Arnhem $(20 \mathrm{~km})$.

Some $75 \mathrm{~km}$ northward from Pannerden, at the northern border of the spacious nature reserve of the Veluwe lies another rural nucleus called Heerde ( 8162 inhabitants). This is the place where Rood Testhouse is located. At the date of the interview in 1988, when the firm was operating very successfully, it was able to recruit all of its lower-level employees (junior technical school level) as well as medium-qualified personnel from the local business area. The same was true for 95 per cent of its highly qualified workers, who had received a higher vocational education. Furthermore, director Weishaupt praised the work ethic of his employees, which he calls "formidable".

Proceeding even further, to the 'barren north' of the Netherlands, one will ultimately cross the boundaries of the province of Friesland, which traditionally has been a peripheral policy region. Its capital, Leeuwarden, which lies only a few miles from the Wadden Sea, hosts another sample firm, the successful synthetics company Casolith Sheets. Despite the status of Friesland as a peripheral problem area, Casolith Sheets is able to find sufficiently suitable personnel within the local business region. The company seeks all its employees mainly by means of employment advertisements in local papers. Spoelstra: "We are perfectly able to acquire our personnel locally. We have not the slightest problem in finding new employees. Why then should we put advertisements in national papers?" Casolith Sheets obtains practically all personnel from within a radius of $40 \mathrm{~km}$. Director Spoelstra: "In 1989 we needed 12 extra employees at once. Over 
100 applicants responded to our employment advertisements, of which 50 per cent were eligible. So, we had a wide choice. We enlisted six people at junior technical school level and six who had enjoyed intermediate teclnical school education. What more could one ask for?" Furthermore, for highly educated staff members, such as production and project managers, Casolith Sheets takes advantage of the Technical College, which is located in Leeuwarden.

Two other peripherally situated manufacturing firms in the sample, i.e. Aalberts Industries (supplier of tailor-made metal products) and Elephant Industries (precious metal dental products) cannot satisfy their labour requirements from the local labour market to the same extent as the businesses mentioned above. Certainly, Aalberts Industries in Venlo is able to attract over 90 per cent of its production-line operatives and intermediate-level personnel (junior technical and intermediate technical school level) from the local business area. But for top workers, this percentage is much lower, viz. 40 per cent (about 20 out of 50 high-quality staff workers). Furthermore, director Aalberts indicates that finding sufficient workers in accordance with the company's potential for growth is becoming a serious problem (Algemeen Dagblad 25-2-1989).

Elephant Industries in Hoorn, which lies in the north of the province of North Holland, not only has to attract all its top personnel (about 10 persons) but also its middle management (also about 10 persons) from outside the local business area. However, director Brouwers does not see this as a major problem. This is mainly because Brouwers perceives the pleasant environment as well as the more favourable housing market in and round Hoorn compared to the Randstad to be of great help in attracting and retaining skilled workers from outside the area.

Roughly speaking, the firms mentioned so far give the impression that, concerning the level of education, the labour market in the Dutch periphery is suitable. Also regions outside of the Randstad seem to be sufficiently covered by appropriate educational institutes for (growing) firms to be satisfactorily provided with skilled employees. And although some entrepreneurs find some shortcomings in the curricula of these institutes (not focussed on practice, ignoring communicative and commercial skills etc.), the basic infrastructure seems adequate. However, when it comes to other aspects of the labour market, such as prior work experience, for example, the situation is less clear. On the one hand, a number of firms in peripheral areas, such as Rood Testhouse, Bronkhorst High Tech, Casolith Sheets, Hulo International, and Nedap, can satisfy the vast majority of labour requirements within the local business region, ranging from low to high-level workers. According to director Paijens of Nedap, this need not come as a surprise, since in the Netherlands manufacturing industry is concentrated in the eastern half of the country. About the western Netherlands he says: "All that is, is trade."

On the other hand, the cases of Elephant Industries and Aalberts Industries show that the capacity of the local business region to provide enough senior staff members and skilled personnel may not be sufficient everywhere in the Dutch periphery to sustain small-firm growth. This impression is reinforced when considering two nonRandstad service firms in the sample which require very highly qualified staff workers. These firms are Intercai (telematics consultancy) in Geleen and System House Comdes in 's-Hertogenbosch. As far as Intercai is concerned, it is unthinkable that the enterprise would have succeeded in finding all its current employees within 
the local business region (i.e. the province of Limburg) if the entire company had remained concentrated under one roof in its original business location. This is all the more true since the percentage of locally derived employees at the Geleen office fell from about 85 per cent to about 66 per cent between 1988 and 1990. This suggests that the capacity of the regional labour market to sustain further company growth rapidly becomes depleted.

With respect to System House Comdes, let us refer to an event already mentioned. Director Vissers of Comdes was able to find only 30 per cent of the 44 newly acquired employees with extensive prior work experience from within a radius of $50 \mathrm{~km}$, in an endeavour to upgrade the organization's activities within the scope of a major reorganization. Contrary to plan, he had to attract the right people from outside this circle, mainly from the Randstad; this amounted to no less than 70 per cent of this type of workers. It goes without saying that this forced reorganization costs upward.

In contrast to the above example of System House Comdes, consider the situation at a Randstad firm like Infotheck. In the 1980s, Infotheek was a very successful, rapidly growing computer trade organization. It was located in Leiden, a city in the western wing of the Randstad. Initially, director Van Leenen, via the Bureau for Academics, recruited extensively unemployed people who had enjoyed either a higher vocational or an academic education. Via additional in-house training, Van Leenen made these people fit to occupy sales functions in his organization. However, after some years, like System House Comdes under the regime of Vissers, Van Leenen cut back on hiring unemployed people at the entry level. "We have tumed away from unemployed people. Now, we search more intensively for people who already have several years of prior work experience. Then we don't have all the bother of extra training". However, this type of workers does not grow on trees. Nevertheless, unlike Comdes, Infotheek succeeds in finding more qualified employees within the business region, that is to say within a radius of $30 \mathrm{~km}$. Van Leenen: "We don't want people who have to travel long distances from home to work. We want our workers to have a lot of time at their disposal for the company, more than eight hours a day. If they had to spend four hours a day travelling, they would not be able to do so." Infotheek intensified the search for more experienced employees. Headhunters were called in, more 'aggressive' advertisements were run in the papers, employee recruitment fairs were visited and mailings were conducted. Furthermore, Infotheek's annual report of 1987 mentioned that the company was able to take advantage of the fact that some automation businesses were cutting back on staff. The company found out about these business contractions via the local contact network of some of its employees. Van Leenen: "Some of our employees were informed that something was happening in these organizations."

Two out of the other three Randstad businesses, i.e. Groenendijk and Waterland Automation, just like Infotheek, are able to derive all the employees they need from within the Randstad. Database Consultancy Intemational in Amsterdam, on the other hand, also hires many employees from outside the local business area, although (financial) director De Zoete is not able to present figures on the region of origin of workers. De Zoete: "Our employees live dispersed, because we have an office in Breda [province of North Brabant], in Utrecht and in Amsterdam. Furthermore, many of our employees do most of their tasks at the client's site." In other words, many of DCl's employees are not required to live near one of the firm's offices.

Anyhow, the above examples qualify somewhat the initial conclusion of only very 
moderate quality differences in the labour market between the Randstad on the one hand and non-Randstad regions on the other. It appears that for particular kinds of activities (business service firms) and occupations (senior level), regional labour market conditions really may be more adverse for peripherally located businesses than for centrally located ones. It should be realized, however, that insofar as some peripheral firms in the sample run up against local labour market constraints relative to centrally located businesses, these constraints have emerged only rather recently. So, it remains that regional labour markets in the Dutch periphery are as able as central ones to sustain both the start-up and early rapid growth stages of the survey firms.

\subsubsection{Summary}

The above explanation evolves around two observations:

(1) First, the businesses studied, both those in the Randstad and those in the periphery, are or were able to make use of the local labour force to a significant extent. This suggests that in the Netherlands inter-regional labour market differences are minor and must not be exaggerated. However, this statement should be qualified in two respects. First, labour market conditions in the periphery seem to be more appropriate for the manufacturing businesses than for the (knowledge-intensive) business services in the sample in their current stage of development. Secondly, the convergence across space of appropriate labour supply for the development of promising high-technology companies applies more to the degree of education than to the extent of prior work experience This observation seems more sound for service activities than for production firms. All in all, highly skilled junior workers are more widely available inter-regionally compared with experienced senior workers. This does not detract from the fact that the businesses in the sample are strongly rooted in the local labour market during their start-up and early growth stages. So, it appears that labour market conditions are sufficiently adequate to allow the launch and take-off of promising businesses in almost every region in the country. Only after these stages of infancy does the more limited capacity of labour markets to sustain further business growth in the periphery, as opposed to the Randstad, become manifest for some firms.

(2) However, the businesses investigated do not resign themselves to the constraints or inadequacies of the labour market. On the contrary, they actively counteract these mismatches in many ways. In this respect, it is revealing that the company which hires the smallest number of workers from the original local business region - i.e. Intercai in Geleen - is one of the best-performing businesses in the sample.

The subsequent sections of this chapter will deal with interactions between firm and environment with respect to resources other than labour. However, these sections will be less extensive than the present one. 
Access to financial capital in general and venture capital in particular is considered by some workers to be of vital importance. It can be conceived of as the linchpin for the transfer and commercialization of new technology and a vital precursor for future economic growth (Thompson 1989). From a spatial point of view, it is commonly accepted that the markets for capital in most developed countries have been successfully integrated into a unified national system. This has occurred because of a number of developments. These include the establishment of many-branched distribution systems of national banks, consisting of numerous small subsidiaries at the local level. But the integration is also the result of progress in communication technology, which has enhanced nationwide access to all major public capital markets. It is believed that as a result of integration, investment capital is equally available everywhere and its price (for a given type of credit) is more or less invariant in space (Gertler 1984). On the other hand, a particular segment of the financial capital industry, i.e. venture capital, is unevenly distributed across space and highly concentrated at existing centres of economic activity (Gibbs 1991). Furthermore, it should be added that the know-how and competence at local offices of large financial organizations are limited. According to Martin $(1989,397)$, the spatial bias in the sources of venture finances is almost certain to exert an independent supply-side effect. This is because venture capital firms based in the core areas are not sufficiently aware of investment opportunities in peripheral areas, and the need to monitor investments restricts funds to a local focus. However, these latter arguments will hardly pertain to the Netherlands, because of its small size. Consequently, it may be safely assumed that firms outside the Randstad have access to appropriate finance equal to that of Randstad-based businesses. In any case, the sample firms did not give any indication of the reverse. Therefore, we pay somewhat less attention to obtaining financial capital compared to the acquisition of other resources.

\subsubsection{Manipulation}

Manipulation mechanisms vis-à-vis the financial environment can be divided into three categories. These are the exertion of pressure on financial agents in the environment, the leverage functions of intermediate agents, and prestige.

\section{Pressure}

The second oil crisis of the late 1970s caused the most serious worldwide economic recession since the 1930s. The Netherlands was particularly hard hit, harder than surrounding countries. By 1981, thousands of businesses were in deep trouble, and the number of jeopardized jobs ran into six figures. Casolith Sheets, a medium-sized company with sales of about 10 million guilders, was no exception. The company was running a loss of two million guilders and bankruptcy was imminent. The new director, Ernst Jansen, undertook the arduous task of trying to save the company. Together with three staff members he drew up a far-reaching restructuring plan; this required investments amounted to four million guilders. The compelling question was 
how to come by this money. Jansen had previously worked for the regional development organization for the northern problem regions (the NOM). He decided to appeal for help to his former employer as well as to the bank. And although the NOM is the sole shareholder of the company, the development organization was not keen to put more money into the operation, especially since earlier endeavours to get the company back on its feet had failed. Nevertheless, Jansen succeeded in acquiring the money; he got three million guilders from the NOM and one million from the bank. When asked how he managed this, Jansen answered: "Because of nty prior work experience, I knew how banks reason, how such a development corporation argues. I knew the best way to talk about financial reconstruction measures; plans must open up new perspectives, however, without falling into the error of being too optimistic." And then he added: "In talking to the NOM, at the same time we told them, 'If you do not come up with the money, we will part as friends. We understand that it is a big risk, as far as you are concerned. But in that case, I would propose closing down the company right now." By threatening the NOM with firm closure, Jansen exploited the political situation prevailing in those days, whereby the government tried anything to preserve existing employment, particularly in problem areas. Jansen: "It is quite clear that the NOM was pressured to invest in Casolith Sheets for purely political reasons. It is unthinkable that a lossmaking business, as Casolith Sheets was at the beginning of the 1980s, would ever be taken over by any regional development corporation at the present time. These organizations have now become far more reserved about putting money into poorly performing businesses."

Jansen is not the only director in the sample who has exerted pressure on financiers by means of threats. Director Van Leenen of the Infotheek Group has also done so.

\section{Leverage}

The term leverage is used to mean that firms indirectly exert influence on the behaviour of environmental elements through intermediate agents. An example with regard to obtaining financial capital is provided by the directors of Rood Testhouse. In the early years of self-employment, Bijsterbosch and Weishaupt found it very difficult to scrape together enough money to sustain the growth of their business. Director Weishaupt: "We looked everywhere for sufficient financial means. The problem was that our company had no track record to inspire the confidence of financiers." After operating in financial straits for four years, an important breakthrough occurred. On the advice of the local chamber of commerce in Zutphen, Bijsterbosch and Weishaupt let the National Bureau of Manufacturing (Rijksnijverheidsdienst, RND) investigate the the viability of their company. The report of the RND served as a crowbar to pry their way into the graces of the (Rabo) bank. With the report of the RND in hand, Bijsterbosch and Weishaupt induced the local subsidiary of the Rabo bank to be much more liberal in lending money than before.

\section{Prestige}

In September 1986, the term of a 20 per cent share of the Brabant Development Corporation (BOM) in equity capital of System House Comdes expired. Comdes bought back the BOM shares, but immediately made a proposal to let the BOM participate anew in the company. The BOM agreed with this, albeit with reservations. Because the BOM is owned by the national government, the approval of the Ministry 
of Economic Affairs was required for this transaction. Sometime between Christmas and New Year, the then Minister of Economic Affairs, Mr. R. de Korte, suddenly decided to cancel the transaction. The reason was that, since Comdes no longer can be considered a risk-bearing venture, the company falls beyond the scope of the BOM, which is expected to invest in promising risk-bearing investments. Because of repercussions for the annual financial report Quekel and Kragtwijk did not want Comdes itself to buy back the BOM shares. Instead, they wanted new share-holders to take over the participation of the BOM before the end of the year. However, the company proved to be very saleable in financial circles, and within three days everything was fixed. Kragtwijk: "They approached us of their own accord."

Similar experiences were recounted by director Jansen of Casolith Sheets. After the reorganization of the firm in the early 1980 s was complete, the profitability of the company increased rapidly. And although the confidence of the financiers was difficult to obtain initially, financing problems eventually vanished into thin air. Jansen: "Financiers are now standing in line to talk to us. It's very funny to see this occurring."

\subsubsection{Immunization}

Firms may not only gain some protection against the inadequacies of the labour market but also against the deficiencies of the financial market. One way is for entrepreneurs to fall back on their own financial resources.

\section{Integration}

At the date of the interviews, financial capital was easy to come by for Casolith Sheets, as indicated at the end of the foregoing subsection. But this was very different in the first half of the 1980s, ever after recovery. Consider the following anecdote. By 1984, Casolith Sheets had recovered and the company was operating at a profit again. Then the NOM indicated to Jansen that the development corporation no longer wanted to be the sole shareholder of Casolith Sheets. The Regional Development Corporation intended to sell its shares. At that point, director Jansen, together with the NOM, set off trying to find other shareholders. They failed, however, in their endeavour. It turned out that its past came back to haunt Casolith Sheets. Jansen: "We were full of a sense of victory, because we had got Casolith back on its feet again. However, potential shareholders did not share these feelings. They mainly looked back to the troublesome past of the company, to all those years it had been operating at a loss. This was very frustrating for us to discover. We reached a stage when we said: all right, then we will take care of it by ourselves. I went to the NOM and told them that we would like to buy Casolith ourselves." The NOM was taken aback by this proposal to let Casolith Sheets be taken over by its own employees. Initially, the Development Corporation considered it a hare-brained idea. After prolonged negotiations it was decided in 1986 that both management and employees of Casolith Sheets would buy 49 per cent of the capital stock from the NOM. Jansen offered every employee the opportunity to buy shares. In the end it turned out that 23 daredevils among the personnel were prepared to put 50,000 guilders each into the enterprise. By the way, later on that year Casolith Sheets also 
applied for quotation on the stock market in Amsterdam. This occured mainly because the NOM insisted on getting rid of its majority participation. Staff members of Casolith meanwhile refrained from acquiring a majority participation. At the same time, Casolith was in need of more investment capital in order to realize its plans to expand the factory. Other firms in the sample, such as Bronkhorst High Tech and Hulo International, rely almost exclusively, apart from government grants, on retained profits to finance the firm.

Considering the degree of (in)vulnerability to the (un)availability of external financial resources, it is also worth noting that out of the nine younger businesses, the start-up of at least four has been either solely or mainly funded by the founders themselves. These sources comprise either personal savings (Comdes, Infotheek) or house mortgages (Intercai, Waterland). Of these, only director Kivits of Intercai indicates that he decided to take out a house mortgage after attempts to obtain money from a bank (NMB bank) and a regional development corporation (LIOF) had failed. The directors of the other three businesses resorted to personal financing from the outset. It is a well-documented fact that over 50 per cent of new entrepreneurs get started with entirely self-generated financial means. The percentage of new businesses which start without any help from banks or venture funds is even higher, since many entrepreneurs receive informal start-up capital in the form of gifts and loans from friends or relations (see for example Storey 1982 and Donckels 1990). Formal investors appear on the stage only after the new enterprise has survived the start-up phase, which takes two or three years (Donckels 1990). This allows them to expand more rapidly. The other side of the picture is, however, that the new entrepreneurs have to accept and deal with external interference.

\section{Rationing}

Apart from internalizing activities, firms may immunize themselves against certain elements in the financial task environment by means of rationing relations with them and switching to other elements. An example of this is provided by director Kivits of Intercai. Kivits considers it very important that he always has had 'good' shareholders, who renounce dividend payments. This makes it possible to pump profits back into the enterprise, particularly into investments in human resources. Kivits considers this of vital importance. Nevertheless, when Intercai was about five years old and had become a rapidly growing company, director Kivits felt that he could not expect the current shareholders, who at that time were local private investors, to keep on deferring payment of current dividends. In 1984 Kivits suddenly found a solution to this problem. In that year Intercai conducted a research project for the Dutch national aviation company, KLM. During the meeting at which the research results were presented, KLM unexpectedly showed interest in taking part in Intercai's stock capital. KLM took this step not for financial but merely for strategic or network reasons. Telecommunications is an extremely relevant activity for an aviation company. In 1985, KLM bought out some of Intercai's current shareholders and acquired 47 per cent of the capital stock. Furthermore, 29 per cent of the capital stock was taken over by members of Intercai's own staff. In the course of the second half of the 1980 s, Kivits succeeded in acquiring two more so-called strategic shareholders, who have no interest in receiving dividend payments from Intercai. These two new participants are PTT Telecom and BSO (a very successful young automation company). So, 
on April 1 1989, Intercai had the following shareholders' KLM, owning 35 per cent of the stock capital, BSO, with 20 per cent, PTT Telecom, with 20 per cent, and Intercai staff, with 25 per cent. It is of vital importance that Kivits was able to arrange that none of these shareholders would make any claims on dividend payments for at least the next five years. In this way, Kivits insulated his firm from the threat of profits draining away beyond the walls of the company, that would have made Intercai all the more dependent upon bank loans and other external sources for financing growth.

In a strictly spatial sense, firms may become independent of the local supply of financial resources by rationing the home region and switching to extra-regional financiers. For example, after the first five years of operation, Intercai began to change its stockholders, at which time the company replaced local shareholders by national ones. When the local financial backing of the BOM was suddenly cut off from System House Comdes, the company had to find new shareholders. At that point, it too turned mainly to non-local investors. One of the new shareholders was Modalfa, a big private investment group from Amsterdam $(85 \mathrm{~km})$, which took over the Comdes Group two years later. Also Elephant Industries has two major shareholders outside of the business region, 1.e. the APPM in Amsterdam and the MIP in The Hague. Since Casolith Sheets has been taken over by the Société Nationale Elf Aquitaine, its equity capital comes from France.

\subsubsection{Adaptation}

A particularly good example of how a company adapts to mandates of banks is the case of Elephant Industries, manufacturer of precious metal dental products. As mentioned above in 1969 director Brouwers started Elephant Industries as a trading business. However, from the beginning it was his intention not only to sell but also to produce precious metal products. So, trade profits were invested in building and expanding the manufacturng capacity. However, the retained profits were inadequate, much more money was required Consequently, Brouwers also appealed to the bank ( $A B N)$ for loans. And although the bank is generally not unwillingly to lend money, the demands of Elephant Industries were far in excess of what the ABN was ready to provide Brouwers "Fmancming could not keep pace with the growth potential of the company There is always the attachment of loan capital to stock capital. You cainnot borrow 100 millon guilders if you only have one millon, at most you can borrow four or five milhon That was our problem in those days Furthermore, venture capital funds did not exist yet in the 1970s In those days mestments had to come from the sphere of credits "To increase equity capital more rapidly in order to obtain larger loans from the bank, Brouwers had to raise profits. To achieve this, he employed the following trade strategy. Brouwers" "I tried to borrow as much money as possible from suppliers who gave me a longer term of payment than usual If the common term of payment was 30 days, I tned to agree on 60 or 90 days. Once, I succeeded in extracting a term of payment of 180 days. From my customers, on the other hand, I strove to receive payments withm either a standard or shorter time interval. In this way I had money at my disposal for two or three months to conduct two or three additional business transactions. The resulting profits were completely recycled into the company [thereby buttressing the firm's assets]. Well, this helped a lot. In 
this manner, bit by bit I was able to receive increasingly larger loans from the bank. This was the way things were going in those days." By the way, this does not detract from the fact that when in 1981 the $A B N$ established a venture capital fund, i.e. APM Ltd. in Amsterdam, Brouwers was there in a flash to request risk capital.

\subsubsection{Utilization}

It is beyond dispute that the businesses in the sample not only have to counteract but are able to benefit from elements of the local financial task environment. Casolith Sheets, for example, would have been doomed to die in the late 1970 s and the early 1980s without the financial backing of the NOM and the local bank. And one might doubt whether Jansen would have succeeded in getting sufficient money for restructuring the company if he had been completely dependent on non-local resources in those days.

For a number of younger companies in the survey the importance of local financial resources appears to apply particularly to the early life of a firm. This holds for both Randstad and non-Randstad companies. Insofar as the founders in the sample do not rely on their own resources for start-up capital, they make use of local financiers, either for equity capital or for loan capital or both. And also local sources remain important some time thereafter.

However, ultimately the non-Randstad companies in the sample have to shift to extra-regional sources, and they do so more intensively than the Randstad companies. A comparison between Aalberts Industries in Venlo and the Infotheek Group in Leiden makes this point clear. When their requests for loans go beyond the competence of local subsidiaries of their bankers, the directors have to turn to the headquarters for new bank loans. For the Infotheek Group, this means that new monetary loans come from Amsterdam, which is at a distance of about $38 \mathrm{~km}$ from Leiden. Aalberts Industries had to switch to Utrecht, which is at a distance of about $148 \mathrm{~km}$ from his company in Venlo. Peripheral area firms like Intercai, Comdes and Rood Testhouse started to retreat from the region for new (stock) resources after four to six years of operation, although there is no indication that the firms consider this to be of any importance. Anyway, Randstad firms, in contrast, remain much longer and much more intensively committed to capital sources from within the business region than their counterparts in the periphery.

\subsection{Managing the material input environment}

Much geographical literature has concentrated on flexibilization and the concomitant need for disintegration and subcontracting of production. The resulting deepening of interfirm transactions and the attendant transaction costs have attracted much attention in regional science. It is assumed that these transaction costs may be spatially determinated to a high degree. Scott (1983, 1988a) contends that where connections between firms are small in scale and rapidly changing, the spatial convergence of interconnected firms is essential if economic efficiency is to be 
maintained. Therefore, it is assumed that dense agglomerations offer important cost advantages to many modern companies in the sphere of subcontracting and input deliveries. On the other hand, isolated companies are confronted with serious disadvantages regarding intensive subcontracting relations. They either have to incur higher transaction costs than firms in the centre or they must adhere to an inflexible, and therefore inferior, vertically integrated organization structure. Theoretically, there seems to be no other way out of this dilemma but relocation of the firm. In spite of this theoretical impossibility of achieving competitiveness in the periphery, successful high-technology businesses in outlying areas actually do exist. This section devotes attention to them and shows how they deal with external material supply conditions.

\subsubsection{Manipulation}

The sample firms have been observed to exert influence on material supplier firms by exerting pressure, as well by means of their reputation.

\section{Pushing and adjusting}

As the gas flowmeters of Bronkhorst High Tech get technologically more sophisticated, they are equipped with an increasing number of electronic components. At the same time, the company strives to further reduce the size of its instruments. To decrease the size while incorporating more components, in the mid-1980s Jouwsma and Bruggeman decided to introduce the Surface Mounted Device (SMD)technique. With this technique, electronic components become fixed to printboards in a different way, which makes it possible to use even smaller components. In addition, the SMDtechnique is able to equip printboards double-sidedly instead of onesidedly with components. However, in those days no firm existed in the Netherlands with a thorough command of the SMDtechnique. Jouwsma: "We were the very first producer of instruments in the Netherlands to apply this new technique, which has become very popular nowadnys." Subsequently, the technical director explained how the problem has been resolved. Jouwsma: "So, we said to our component supplier, LM Electronics in Neede [13 $\mathrm{km}]$ : 'We want to move in the direction of SDMtechnique too.' They answered that they were not able to do it. Then I said: 'Just make sure you figure out how to do it. It las to be done.' Then we went to Germany together, visiting firms which already had started making themselves familiar with the technique." Apperently it was not an easy task; it implied quite a revolution for LM. New apparatus had to be purchased to realize the job. LM hesitated whether it should proceed. Jouwsma: "Well, in the end they did pick it up, because we simply said: You have to. We need the back of those printboards for fixing more components; the front is fully covered already. So we cannot proceed any further, technologically we have reached a ceiling." By the end of 1986, LM had done the job and Bronkorst introduced the SMDtechnique into its instruments.

In 1988, a similar situation occured. The electronic flowmeters which Bronkorst produces are endowed with temperature and pressure sensors for measuring mass flows. When the company took up the development of a new type of flowmeter, it also decided to build in a new, cheaper, type of sensor. This new sensor would be developed on the basis of a 'thin membrane technique' instead of the conventional 
winding technique. In the Netherlands, Jouwsma could not find a supplier with the know-how to produce the new sensor. Finally, Jouwsma found a small company in Germany, in the Black Forest, that was able to develop the new sensor desired by Bronkhorst. Jouwsma: "The German company was able to construct the sensor exactly as we would like to have it." Nevertheless, Jouwsma decided not to take a chance with the Germans. Jouwsma: "It is too far away from us after all. We want to lave it closer by our firm, that really works better." Jouwsma decided to have the required sensor developed by a current supplier located in Doetinchem $(22 \mathrm{~km})$. Together with a staff member of the Doetinchem company, Jouwsma paid the German firm several visits in order to copy their production technique. Jouwsma on the Doetinchem producer: "At any rate they have mastered the basic principle. The only snag is that they still have to learn how to apply it exactly. But that will turn out alright, it's only a matter of time."

Furthermore, Jouwsma sees to it that when suppliers invest in new equipment they take into account the demands of Bronkhorst High Tech. Jouwsma: "The supplier, a metal-working company in Vorden $(12 \mathrm{~km})$, has a numerically controlled machine in house, with which everything can be done. It is able to manufacture a complete product in a single run. These are impressive investments. Such machines cost from 500,000 to one million guilders. But we have made sure that he meets our requirements in his apparatus as well. We told him: mind that you take into account such-and-such..., the machine has to meet this-andthat contition. Not that he buys just for me, he uses it for others too." These examples show that the relations between Bronkhorst and its suppliers are characterized by intensive interaction and a concomitant mutual influence. This results from the firm's hollowization strategy. This implies farming out actual production, not on spec but rather in cooperation with suppliers.

\section{Reputation}

The computer dealer Infotheek and the software engineering firm Waterland Automation also influence the domain of potential (hardware) suppliers. But they do so in a different, less direct manner; namely, through their reputation. After being in operation for respectively three and five years, both Infotheek and Waterland were approached by the Dutch branches of reputable international hardware manufacturers with an offer to market and develop software for their machines. Infotheek was approached by IBM located in Amsterdam $(38 \mathrm{~km}$ ), and Waterland was contacted by Digital in Utrecht $(52 \mathrm{~km})$. For both companies, this was an important breakthrough, as it gave them access to large markets. Director Schoneveld of Waterland Automation discloses how this came about. "We started Waterland Automation as a system house for Data General computers. And now we still purchase Data General computers, we develop software for them and produce turn-key systems for the end-user." By the way, Schoneveld's connections with Data General date from his previous job at Stork in Amsterdam (metal products). Stork used Data General computers. Schoneveld: "We have been appronched by Digital on the basis of our success with developing software for and implementation of Data General hardware. By that time, as a system house, we had become the largest customer of Data General computers." 


\subsubsection{Immunization}

\section{Integration}

When elements of the external environment cannot be brought in line with a firm's requirements, the company may choose to integrate these elements in some way. Consider, for example, the remarkable step taken by Casolith Sheets in this respect. After the company was drastically restructured in the early 1980s, it entered into a stage of steady growth. Casolith performed even better than its rivals. Consequently, the Leeuwarden firm occupied an ever-increasing portion of the European market for acrylics products, from two per cent in 1980 to seven per cent in 1986. However, the synthetics company has one weak point. Unlike its main rivals, such as Röhm in Germany, ICI in England and, to a lesser extent, CdF in France, which have polymer acrylics products in their assortment too, Casolith does not have its raw material supplies in its own hands. This makes the company vulnerable in comparison with its competitors. There is a threat that competitors will eventually conspire to cut Casolith off from raw materials, such as 'monomer', which is a liquid. In order to ensure his supply of raw materials, Jansen intended to sell to a supplier of raw materials the 25 per cent of equity capital which the NOM still owned after the introduction of the company on the stock market. However, things did not take place the way Jansen had in mind. In Spring 1987, a mere seven months after Casolith was first quoted on the stock market, the French state company Chemie de France S.A. (CdF) got in touch with the Frisian producer. It appeared that the French company, which is an important supplier of Casolith Sheets, wanted to buy up the entire firm. If Casolith did not agree with this offer, CdF would set up a factory similar to Casolith. After consultation with his commissioners, Jansen decided to agree with the proposal of CdF. His main reason was to guarantee the supply of raw materials, which is the company's achilles heel, as Jansen puts it.

Another example of how a company may immunize itself against the contingencies of suppliers in the environmental domain or task environment is provided by director Jouwsma of Bronkhorst High Tech. Surprisingly, in the second interview, conducted in 1990, Jouwsma indicated that the idea of subcontracting its entire production, which the company has adhered to since its establishment, is losing ground somewhat. Jouwsma intended to internalize the production of certain components and the performance of some tooling techniques typical of Bronkhorst High Tech in the future. The reason is that suppliers do not apply new techniques as rapidly as Jouwsma would like them to. Jouwsma: "This is because they have to look after other interests as well. They not only deliver to us, but also to other firms." For certain orders, subcontractors take too much time, or these assignments are not done very well. Jouwsma provides two examples of activities which he considers internalizing in the future. The first is 'electro polishing'. Jouwsma: "This is a very special technique, characteristic of our firm." The subcontractor does not provide the same high quality unremittingly, and Jouwsma expends much energy to get him to do so. The second example is the production of 'prints'. Jouwsma would like the subcontractor to change the arrangement of SMDcomponents on the printboards. Jouwsma: "He does do it for this draft, but not for the next one. So, I have to start all over again. One is too dependent. Our philosophy for the future is that we will retain specific knowledge in-house. 
And perhaps we will go so far that we do it for others as well."

The fortunes of Elephant Industries, producer of precious metal dental products, are vulnerable. Obviously, they are dependent on the price of precious raw materials such as gold, palladium, platinum, and silver on the international market. But since the prices of precious metals are expressed in U.S. dollars per troy ounce $(31,1035$ gram), they also fluctuate with the exchange rate of the American dollar. In order to lessen this dependency, Elephant Industries has invested in a unique method of refining their waste of precious metal alloys with the help of an ultramodern refininginstallation. This waste material is produced during the processing of precious metal alloys. The refuse consists of snips, scales, filings, grindings and residuals. In this way the company is able to achieve a greater degree of self-sufficiency regarding raw materials (Annual report 1986).

Director Bijsterbosch of Rood Testhouse provides us with the last example of immunization against environmental barriers with respect to material supplies by means of internalization of activities. Initially Bijsterbosch and Weishaupt did not have enough money to buy all the test devices they required. Bijsterbosch: "In the beginning, test apparatus was sometimes too expensive for us. Then we had to look for other options and we developed parts of our equipment in-house."

\section{Buffering and scattering}

The Infotheek Group faces serious difficulties with irregular and late supply of computer hardware (particularly IBM computers), which the firm sells and installs. One of the measures taken to buffer against these irregularities is to keep hardware in reserve in order to put at a client's disposal temporarily.

Yet another strategy by which businesses in the survey immunize against the material supply task environment is by scattering relations. After the take-over of Casolith Sheets by CdF Chemie, the Leeuwarden company was no longer in a position to buy its most important raw material, monomer, independently. However, other supplies such as glass, pigments, PVC and the like are still bought autonomously. With respect to these products, Casolith diminishes its susceptibility to suppliers through multiple sourcing. The company sees to it that every type of material is provided by two or three suppliers.

\section{Rationing}

When Bruggeman and Jouwsma started Bronkhorst High Tech, they decided not to undertake the actual production of the various components of the flowmeters by themselves but to subcontract these activities instead. The two men confined themselves to research and development as well as the assembly of the instruments. The reason for this 'strategy' is that when the founders decided to start their own company, they did not envisage it raising sufficient money to set up an adequate production capacity. Jouwsma: "Even just a modern lathe, which is a very advanced machine nowadays, costs half a million guilders. To this, tools running at 300,000 guilders have to be added as well. For a new enterprise, it is impossible to cope with this. So, we decided to invest not in means of production but in human resources and in research and development." In this way Bruggeman and Jouwsma avoided entry barriers in their line of business.

Although director Brouwers prefers managing a manufacturing firm, he set up 
Elephant Industries as a company trading in precious metal products. Brouwers: " $A$ trade business did not really appeal to me much." The reason for starting Elephant Industries as a trade enterprise was to avoid the huge capital investments required to set up a manufacturing company. However, Brouwers did not abandon his intention to operate a production firm. Using the profits from his trade activities, Brouwers succeeded in the course of time to develop Elephant Industries into a full production company.

From a spatial point of view, with respect to acquisition of material inputs, most firms in the sample operate rather independently of the local environment. Groenendijk in Ridderkerk buys its cabins from firms in Coevorden (198 km), Raamsdonkveer $(48 \mathrm{~km})$ and Nijkerk $(97 \mathrm{~km})$. Aalberts Industries obtains only two per cent of its input materials locally; 50 per cent comes from the rest of the Netherlands, and the remainder from abroad. Director Aalberts: "We don't do anty business in the region." This is also true for the apparatus manufacturer Nedap in Groenlo. This firm buys about two-thirds of its input materials from abroad and one-third within the Netherlands. The domestic portion comes entirely from outside the local business region. An important domestic supplier for the company is Philips in Eindhoven (120 $\mathrm{km}$ ). Casolith Sheets buys its raw materials, which constitute up to 60 per cent of total purchase costs, abroad. Machinery and equipment, however, are purchased domestically from all over the country, although there is a particular emphasis on the business region. Similarly Elephant Industries and Rood Testhouse obtain their input materials mainly abroad.

In sum, many firms in the survey acquire their input materials outside the business area. This is surprising, because some of them are engaged in custom-made production, such as Hulo International, Aalberts Industries and Casolith Sheets. The comments of some of these entrepreneurs on this situation are revealing. When director Westendorp was asked where the main suppliers of Nedap are located, he answered: "Don't ask me where it all comes from. To be honest, I don't really care. As long as everything we need gets here." Director Meeus of Hulo International responded in a similar way: "Oh, I don't know. Suppliers are located all over the country and in Germany." I asked director Groenendijk whether transport costs of make-shift cabins from so far away affect profits adversely, and he said: "Oh, it isı't so bad." Director Bijsterbosch of Rood Testhouse admits that if he could obtain test equipment - which now has to come from all over the United States and increasingly from Japan - closer by, this would be lucrative to his firm; "For us that would be very nice." However, he stipulates one hard and fast condition: "This enterprise would then have to cover the world market. After all, test equipment must be above all suspicion and doubt of customers." These remarks put into perspective the significance of the distance factor for subcontracting practices. In any case, they show that spatial propinquity is not a prerequisite for achieving flexibility. This contrasts with the theory of the geography of flexible specialization, which emphasizes the role of the distance factor in this respect. The remarks, especially that of Bijsterbosch, indicate that other forces, such as product quality and product differentiation, may thwart the supposedly important role of spatially related transaction costs in subcontracting practices. 


\subsubsection{Adaptation}

In order for Bronkhorst High Tech to achieve sufficient flexibility in the market place through custom-made production and just-in-time delivery, its supplier firms must be able to produce and deliver products and components on demand within a week. Director Jouwsma: "We order today what we will need in a week from now." To enable the suppliers to meet this requirement, Bronkhorst's purchasing policy was adapted to include an integrated planning system. This means that subcontracting takes place in two moves. The first step is when Bronkhorst places orders with its suppliers for 'basic supplies' one year in advance. The second step is when orders are placed for specific components and subassemblies in the short term. These have become relatively quick for the subcontractor to produce, since basic supplies are on hand. Jouwsma: "So, we always take care that the subcontractor has a basic stock of supplies for our instruments." For Bronkhorst it is crucial to plan carefully in advance which components are needed by the subcontractor. Therefore, the firm makes huge investments in automating material resource planning, to which we will return in the next section. Jouwsma: "You can't just tell them, 'sorry, I forgot to pass something on to you."

Rood Testhouse is forced carefully to plan the supply of test apparatus in advance for a different reason. The company obtains these tools mainly from producers in the United States, i.e. Terradine and ALX. Weishaupt: "Because these instruments are not available from stock, sometimes very long delivery times occur; half a year is not unusual. This is quite a long time, considering that the economic life of this apparatus is no longer than four years". In order to secure maximal return on the machines, Bijsterbosch and Weishaupt plan their input requirements at least six months in advance.

\subsubsection{Utilization}

The foregoing might have suggested that all the firms in the sample are indifferent to the location of their suppliers, but this is not the case. Bronkhorst High Tech obtains input materials, worth over 80 per cent of purchase costs, from two major suppliers, which are located at a distance of eight and $15 \mathrm{~km}$ respectively. If one of these two firms were to relocate at a distance in excess of $30 \mathrm{~km}$ from Bronkhorst, Jouwsma would drop it as a supplier. After all, "within the local region sufficient alternative potential suppliers are located", according to the technical director. The location of suppliers other than the two major ones is less sensitive to distance. Furthermore, Bronkhorst exploits the local environment in a more insular way. Initially he farmed out manufacturing activities. But in the near future, Bronkhorst intends to internalize the production of firm-specific components. Jouwsma: "we conduct initial development externally; then, at a certain moment, when we have heard enough, we make things part of our company."

Whereas Casolith Sheets obtains many pieces of machinery, installations and other operating inputs from outside of the business area, an important input comes from within the local region: stainless steel machinery. Casolith can extract that equipment from the local dairy industry. On the other hand, both director Jansen and director Spoelstra give us the impression that they would not care a bit if they had to 
purchase machinery from elsewhere in the country.

All in all, it appears that Scott's model of the geography of flexible specialization is not brushed aside by every firm studied. Particularly the spatial subcontracting relations of Bronkhorst High Tech run counter to the above conclusion (under the heading 'adaptation' in this section), which undercuts the role of spatial propinquity for transaction costs. And the reason is clear; Bronkhorst is perfectly suited to the flexible specialization and industrial district model. The firm subcontracts the actual manufacturing of its entire flexible product range. (Recall that the company undertakes only development and final assembling activities). Other manufacturing firms in the survey engaged in tailor-made production are either completely (Hulo International) or partially (Casolith Sheets, Aalberts Industries) more vertically integrated than Bronkhorst High Tech. So, the latter's subcontracting relations are much more intensive and varied than those of the former two. Anyway, director Jouwsma gives the impression that Bronkhorst attaches great value to spatial proximity (i.e. less than $30 \mathrm{~km}$ ) of his main suppliers, although no proof is given that Bronkhorst High Tech would have performed less well if subcontractors had not been found within the local area.

What is striking is that Bronkhorst and, to a lesser extent, Casolith Sheets, both located in the most peripheral and rural areas of the Netherlands, can obtain so many input materials from the local business region. This illustrates that the subcontracting conditions in the Dutch periphery need not be unfavourable, not even for a hollow, high-tech corporation. This finding confirms the contention of Horvers and Wever that interregional differences in economic conditions in the Netherlands are relatively small (Horvers and Wever 1989). Praat (1992) even concludes that for manufacturing industry, subcontracting conditions are actually better in some peripheral areas, such as the southeastern part of the country, than in the Randstad.

\subsection{Handling the business service environment}

The phenomenal growth of business service functions in the past two decades has been accompanied by increasing attention and research from the field of economic and regional sciences. It is believed that many producer services in general make a vital contribution to the efficiency and performance of economic life. Their importance is all the more stressed in this time of continuous transformation of markets, rapid and ever more complex technological change, and the trend towards greater product differentiation. Producer services assist enterprises in coping with the dynamics of the macro-economic turbulence. They help to adapt skills, products, processes, and even attitudes to changes in the environment, or to reduce the organizational, managerial, and informational barriers to adjustment (Marshall et al 1987).

Producer service activities thus play an important role in promoting the development and adjustment of regional (and national) economies to structural changes in society. Accordingly, regions in which these functions are poorly represented, i.e peripheral regions, are likely to become constrained in their economic growth. According to Goddard (1978), the ability of regional industry to adapt to market changes and to develop new products and production techniques is partly determined 
by the availability and quality of local service information and advice. Therefore, independent companies in peripheral regions may be disadvantaged if their local area has a poorly developed service sector (Marshall 1982, 1523; Daniels 1987).

O'Farrell and Hitchens (1990) suggest that firms in peripheral regions with a poorly developed producer service sector may react in four ways. They may simply decide to carry on without the benefit of the service input, thereby creating a latent demand; they may search outside the region; they may try to provide services inhouse; and they may invest in areas with a high-quality service environment (serviceled regional development). Furthermore, O'Farrell and Hitchens suggest that in addition to forsaking services, the most frequently occurring response of small and medium sized enterprises is buying in from outside of the local region. Internalization of service functions is only possible for larger firms.

O'Farrell and Hitchens refer to Marshall's (1982) finding that in the British case, a high proportion of a firm's demands (i.e. 80 per cent of external service inputs) are met locally; if not available there, firms tend to purchase from London. Similarly $\mathrm{O}^{\prime}$ Farrell and O'Loughlin (1981) demonstrated that in Ireland, peripheral firms at greater distance from regional centres tended to skip the regional centre and place their orders in Dublin. However, according to $O^{\prime}$ Farrell and Hitchens, high extra costs are attached to the provision of services over large distances, which hampers such behaviour. They quote Daniels $(1987,5)$, who states that "unless these [i.e. producer services] are available locally the cost of obtaining them ... may be a sufficient deterrent to limit utilisation or, more seriously, to prevent use altogether and so cause firms to grow more slowly or even to cease production because of their inability to compete with those firms located in cities with more immediate access to a comprehensive pool of intermediate services." The extra costs peripheral area firms have to incur in obtaining eligible business services, which Daniels has in mind, concern travel time to meetings, frequency of contact, telecommunication charges, the possibility of delays caused by the inability of the client and supplier to meet as often as required, or simply lack of knowledge. Similarly, O'Farrell and Hitchens (ibid, 1147) themselves establish that when peripheral Irish firms use Dublin-based producer service companies because of local gaps in service supply, the extra costs consist primarily of managerial time and management costs.

All in all, it is perceived that the small firm sector remains very dependent upon the location and local availability of external service inputs. Therefore, it is suggested that firms which are located in remote areas with an inadequate service sector have an insurmountable competitive disadvantage, whether or not they try to resist their environment. When they react against this inadequate supply infrastructure, they incur extra costs, and when they defer counteraction, they incur differential disadvantages.

Against this theoretical background, in the following section we will make an inventory of the reactions of the firms in the survey vis-à-vis both the opportunities and deficiencies of their business services production environment. 


\subsubsection{Manipulation}

\section{Leverage and pressure}

Modern production methods, such as just-in-time production and integrated planning systems, call for careful material resource and time planning in organizations. Bronkhorst High Tech resoundingly confirms this. Director Jouwsma: "It is of vital importance to us that we have the flow of materials and scheduling under control". To that end, Bronkhorst purchased an IBM 36 mini-computer system at a price of about 200,000 guilders. Furthermore, Bronkhorst requires suitable software, both for bookkeeping and for production control. Furthermore, Jouwsma wants these two systems to be linked. Jouwsma: "For example, as soon as an instrument has been sold, stock registration and time planning should be adapted automatically." For that purpose, IBM refered the firm to a software company in Diemen that is supposed to have developed the appropriate software package for this problem. Bronkhorst approached the Diemen company and, according to Jouwsma: "all our desires and wishes were thoroughly discussed. They assured us that their computer program was able to deal with all our needs." However, when installing the system, which had to be adapted at some points to the specific context of Bronkhorst High Tech, problems arose and things did not work out as expected. Jouwsma: "Whereas the system should have been working since last July, now, on the first of March 1988, it is still not functioning adequately. We have not bought the system in its current condition. It does not work as we would like it to work. At the demonstration, which was organized for us before we bought the system, it all went without a hitch, and all our wishes could be met, it was said. But afterwards it tumed out that time planning, as we would like to have it, cannot be dealt with." At the date of the first interview with Jouwsma, the project was in an impasse. However, Jouwsma was not inclined to leave it at that. "They will have to improve it." Via calling for IBM, he intended to put the Diemen company under pressure. "Let IBM come sit right here at my desk and tell me what we should do." Furthermore, Jouwsma has another card in his hand which he can play to try to force the software firm into compliance: "I have paid only two-thirds of the total price; one-third still has to be paid, and that is quite a lot of money".

A second type of producer service which Jouwsma is not content with is advertising, although the problems in this sphere are by no means as serious as those with software engineering. About advertising agencies Jouwsma says: "Advertising services are also such empty words. There is much to be improved in the performance of these agencies. We have to look after the design of our brochures carefully. Otherwise they make very beautiful pamphlets, it is true, but they are useless for selling flowmeters. All that matters to those advertising guys are nice pictures. But first of all, flowmeters have to be sold, and that's what they tend to forget. Once they designed a folder of which everybody was wondering what it was about. We told them that it was no use to us. Look at this one, you can hardly see that it is about flowmeters. The type of letters used has to be changed. Above all, it has to be clear that it is about flowmeters. Those advertising boys are difficult to keep in line."

In a similar way, the Rood Testhouse management seeks to keep a tight rein on their advertising. Director Weishaupt: "We do not lenve things completely to this kind of business. We regularly have to check their products against our own basic idea. It is a matter of visiting them regularly and working on them to keep them in line with our own view." 
The interviews provide few further examples of influencing supporting service firms in response to insufficient deliveries. However, this does not imply that everything is clear sailing. Directors mainly mention two other reactions in answer to inadequate external service deliveries: integration and changing external suppliers.

\subsubsection{Immunization}

\section{Integration}

On 9 January 1990, two years after the first interview with director Jouwsma had taken place, I returned to Bronkhorst High Tech for a second interview. It appeared that problems with tailoring the standard software package to the desires of Bronkhorst High Tech had still not come to an end. Director Jouwsma indicated that he was fed up and that he intended to change his tack. Jouwsma: "Now we are going to see whether we can find new eligible software on the market. Subsequently, we will make specific adaptations to our flexible production system ourselves. If we can find such a system, we will throw out the present one. One must take into account that in those days, when the present company [i.e. the software engineering company from Diemen] started this project, no good computer program in this field existed yet. Since then, developments have occurred very rapidly in that line of business. Perhaps a new computer program has meanwhile come onto the open market which would suit our firm. Then, we would buy the basic program and subsequently develop firm-specific adaptations by ourselves. By the way, we insist that the Diemen firm finish the project. But it remains to be seen whether we are actually going to use it."

When I asked the Nedap managers whether they farm out or undertake software engineering by themselves, director Westendorp answered: "We produce all our software ourselves. In the past we have tried to delegate software engineering to external firms several times. However, we found that the packages delivered did not always fit in with our requirements, nor were they always easy to use." Actually, Nedap had tried out three software companies, two from the Randstad and one from within the local business region, before the management decided to design software internally.

Director Brouwers of Elephant Industries decided to design advertising pamphlets in-house after having had negative experiences with external advertising agencies. Brouwers: "In the past we had a burean that looked after these things for us. I must tell you that we had to very carefully check the quality of the work which these people were able to provide. We fairly often felt disappointed. It mostly proved that our assignments were too demanding. Now we have a department ourselves which takes care of the design of advertising pamphlets."

\section{Rationing (and integration)}

Some firms show how they, by virtue of their smallness, maintain limited relations with (elements of) the service environment and therefore are (temporarily) shielded from possible contingencies in these markets. This has been observed with respect to advertising agencies. Director Schoneveld of Waterland Automation indicated in the first interview, in April 1988, that he felt it was not necessary to advertise intensively. Schoneveld: "I have already indicated that the growth of the firm is dampened because of 
problems in finding suitable personnel, not because of difficulties in finding clients. We are approached by more clients than we can serve." Furthermore, Schoneveld thinks that: "It is very difficult for advertising agencies to transmit one's own ideas to the market." In the second interview, in 1990, the situation with respect to marketing efforts had changed. Schoneveld: "We have paid quite a bit of attention to the marketing of our products." These activities are performed by the marketing communications department of the firm. When asked why these services are provided internally instead of externally, Schoneveld answered: "Because we are not engaged in mass production, we do not sell standard products."

Also Intercai, at least for the first nine years of its existence, did not undertake extensive marketing and advertising activities. This seems not due to inadequate external support, but rather to weak external competition. Donders: "It is still the case that Intercai does not have a professional marketing system. This is still the responsibility of our consultants in the field." By this statement, Donders suggests that Intercai as yet does not need professional marketing assistance, be it either an internal or an external service. Donders refers to the monopoly position which Intercai still holds in the Dutch market for telecommunication consultancy. So, marketing and public relations are allowed to operate on a less professional level. However, when the threat of increasing competition eventually picks up, then the company will have to reach out for more professional marketing services. This can be built up internally or called in externally. By expanding the current management team to include a commercial director in 1991, Intercai seems to have chosen the first option. However, it must be noted that Intercai is still not experiencing serious rivalry from its competitors.

A similar situation is found at Database Consultancy International in Amsterdam. Database Consultancy International does not call in an external marketing bureau. Director De Zoete: "Until recently we had never produced a folder. There was no use for this. This is changing, however, since the firm is growing bigger and bigger."

From a sheer spatial point of view, firms can immunize themselves against constraints in the local business service environment by withdrawing from the region and switching to the extra-regional domain. For example, initially System House Comdes was out for maximal self-financing. The local accountant, who is located in Zaltbommel, supported this view. However, when the company began to grow consistently rapidly, Comdes was recurrently confronted with liquidity problems. In order to solve this problem, according to Kragtwijk, Comdes had to introduce a new business philosophy. This new philosophy abandons the view of maximal selffinancing. Kragtwijk: "So, we needed a good accountant who agreed with this new growth ideology and who would be able to convince external financiers. Our own accountant at that time accorded only reluctantly, which was not in proportion to the investments we made. Then, we decided to switch to a nationally operating accountant, TRN in Rotterdam" [80 $\mathrm{km}$.

Casolith Sheets contracts out supporting services mainly to the local region. For example, public relations services are ordinarily farmed out to a bureau in Drachten $(26 \mathrm{~km})$. However, when Casolith applied for quotation on the stock market, advertising activities were farmed out to a firm in Amsterdam $(138 \mathrm{~km})$, which possesses experience in the financial press. Director Jansen: "Look, this is typically a world in which no experienced people can be found in this part of the country." Similarly, whereas ordinary legal advice is put out to consultants in Leeuwarden, for special, important 
events these activities are contracted out to an enterprise in Rotterdam (205 km). Jansen: "For daily business we have our house lawyer here in Leeuwarden. When considering quotation on the stock exchange and for the take-over of our firm by the French, we consulted a well-known legal consul in Rotterdam, because I think that for such kind of specialities one has to got to the highest place. One can safely have an operation on a finger in a hospital here in Dokkum. However, as soon as they are going to pry into my brains, I would prefer to go to an academic hospital."

Nedap in Groenlo regularly farms out advertising activities, although many activities in this sphere are provided inside the firm by the PR department. Westendorp: "Now and then marketing bureaus do something for us. Printing and the like is performed by businesses here in the neighbourhood. Brainpower activities, on the other hand, are located in the western part of the country."

Director Aalberts of Aalberts Industries gives a similar evaluation of local business services. Aalberts: "Supporting services are not only located in the vicinity of the firm, but also outside of the local business area. It depends purely on which type of services one needs. If one seeks important legal counsel or fiscal advice, one cannot find these services here in this area but has to go to the Randstad. Over there one has much wider choice of businesses and agencies. It depends very much on the size of the firm. Look, a small firm of ten employees will have much less need for important professional services than, for example, our own group of firms has."

Bronkhorst High Tech switched to an advertising agency in Voorburg (182 km) after it had unsatisfactory experiences with an agency in the immediate vicinity, located at a distance of only $200 \mathrm{~m}$ from the firm.

It is clear that mainly non-Randstad firms (have to) farm out certain supporting business services to firms outside of the local business area. The opposite, whereby a Randstad firm uses extra-regional business services, occurs only incidentally in the sample. An example is Groenendijk plc, which farms out software engineering to a software firm in Eindhoven $(98 \mathrm{~km})$. Director Groenendijk: "This occurred purely by accident. In a trade journal, an article was written about automation in a contpeting firm. The software company which implemented this project was located in the Eindhoven area. And via this firm we ended up at Philips computers for computer hardware equipment." Furthermore, another Randstad firm in the sample, i.e. Waterland Automation in Purmerend, contracts out international marketing services to IDC in Brussels.

All in all, it appears that the firms studied deal with perceived or actual inadequacies in the local service task environment by means of hedging against these deficiencies rather than by influencing and changing the elements of the environment. Furthermore, it appears that information about these strategies mainly has to be gathered from the interviews with the directors of non-Randstad firms in the sample. The difference between Randstad and non-Randstad firms is particularly notable with respect to extra-regional service linkages. The latter obtain business services from a much wider area than the former.

Examples of adaptation behaviour in response to barriers in the service environment could hardly be found. Therefore, the next section proceeds with opportunities for utilizing the local pool of business services. 
Examples of resistance strategies in response to inadequacies in the local business service environment are mainly found among the non-Randstad firms in the survey. Nonetheless, these firms, sometimes quite unexpectedly, can take advantage of the local environment as well. This is clearly illustrated by the case of System House Comdes. Though the company has to look to the Randstad or other areas for the provision of certain services (accountancy, international marketing), many activities can be conducted locally. Consider the following example. When Vissers took charge of the enterprise in 1989, he broke off relations with almost every one of the firm's prior service businesses. Vissers started calling in more professional business service firms. Unlike the acquisition of more qualified staff workers (see Section 5.2.4), he was able to find eligible service firms within a $50 \mathrm{~km}$ radius. Bookkeeping, legal advice, advertising, public relations, headhunters; all these activities were found within a radius of $50 \mathrm{~km}$. For accountancy services, Vissers switched from the TRN Group (at present TRN Bakker en Versteegh) in Rotterdam to Coopers \& Lijbrand Dijker van Dien in Arnhem $(62 \mathrm{~km})$. Vissers himself appeared to be surprised that he succeeded in finding suitable service bureaus in the business region so easily. About farming out supporting advertising activities, for instance, he said: "It would have been obvious if I would have tumed to an agency in Amsterdam, since the majority of these activities are concentrated there."

Non-Randstad areas in the Netherlands sometimes have an unexpected capacity to provide business service functions. This is also shown by Casolith Sheets. Just like Bronkhorst High Tech, Casolith Sheets was confronted with problems in regard to software engineering and services in the middle of 1980s. Jansen: "We did try out many software firms. We have had bad luck in selecting these firms. They always went bankrupt or.... Among other things we have been with a bureau from Amsterdam. They are the worst scoundrels I have ever met, awful." Casolith dropped the Amsterdam company and called on a northern subsidiary of the software activities of the travel agency Holland International. When the automation activities of Holland International were taken over by Ormer, an English group, Casolith went along to Ormer, which operates in the Netherlands from The Hague $(182 \mathrm{~km})$. Ormer, however, also went bankrupt. Then, the computer scientists of Ormer, who actually developed the computer software for Casolith, moved to a firm that does not use Philips computers. For this reason Casolith Sheets did not go along with them. Via coincidental informal relations, Casolith ended up at the computer office of the Dairy Federation in its place of location, i.e. Leeuwarden. Decline in the dairy industry forced the Federation to orient itself towards alternative opportunities outside of this line of industry. To Casolith it was important that the Dairy Federation used Philips computers. Jansen is very content with this situation. "Evenything is going excellently."

Similarly Bijsterbosch and Weishaupt of Rood Testhouse returned to the local business region after having tried out several advertising agencies in the Randstad. Weishaupt: "We have had quite a few advertising agencies, and ultimately we ended up at an agency in Apeldoon $[20 \mathrm{~km}]$. After all, there is a need to have one's satellites in the close vicinity of the firm, particularly advertising agencies. We regularly have to check their products against our own basic iden. It is a matter of visiting them regularly .... Well, and 
Just like the advertising agency, the firm's accountants, Moret Ernst \& Young, are also located in Apeldoorn. About accountancy services Bijsterbosch says: "At present, since we are in smooth waters, it has become less important that the accountant is located in the vicinity of the firm than in the beginning. It depends on the growth stage the firm is in. In the starting period it is more important to have the accountant close by than now. Furthermore, it was also handy that the accountant was located nearby when we applied for quotation on the stock market, because everything had to be verified, and hence contacts occurred very frequently." Rood Testhouse can also get legal advice in the local region, i.e. from a local notary office in Heerde.

Aalberts Industries had its accountant and notary initially in Eindhoven $(55 \mathrm{~km})$. Later on, in 1985, the company switched to a national register accountancy firm, Moret Emst \& Young, in Maastricht $(71 \mathrm{~km})$, which in 1989 also established a subsidiary in Venlo. Up to 1980, notary services were provided from just outside the business region. Thereafter, Aalberts switched to a national notary office in Amsterdam $(181 \mathrm{~km})$, i.e. Stibbe, Blaisse \& de Jong.

Intercai is a real do-it-yourself firm with respect to supporting business services. Nevertheless, those activities which the company does contract out lie within its business region. For example, accountancy is provided by TRN Bakker \& Versteegh in Maastricht $(15 \mathrm{~km})$ and incidental consultancy activities by a firm in Heerlen (15 km).

The same applies to Hulo Intemational. The company performs its software engineering, advertising and public relations activities internally. The few services which it does farm out are found in the local business area. Accountancy was provided initially by Moret, Ernst \& Young from Nijmegen $(18 \mathrm{~km})$, and later by the TRN Group from Arnhem $(20 \mathrm{~km})$; legal advice is also found within the business area. Also Bronkhorst High Tech is able to find some important business services within the production environment. This is the case for accountancy (Zutphen $19 \mathrm{~km}$ ) and notary activities (Vorden $12 \mathrm{~km}$, and Zutphen).

The above examples of opportunities for peripherally situated firms to employ business services from the local area do not change the fact that their counterparts in the Randstad can make more extensive use of their immediate surroundings in this respect. The service activities these firms farm out can almost entirely be bought in from the regional production environment, if not from the place of business itself. As an illustration, consider the answer of director De Zoete of Database Consultancy Intemational in Amsterdam when asked if he thinks that opportunities for small-firm growth are better in the Randstad than elsewhere in the country. De Zoete: "If we weed a good tax consultancy business, well, we can find it upstairs in this same building. When I want to talk to my lawyer I can find him right around the comer. And this is what we always consider carefully; to make sure that we call in good consultants, the best one can get in the Netherlands. Well, and they are located here in these very neighbourhoods [i.e. the Amsterdam canal district]. If they would be located outside of Amsterdam that would be very difficult, because these kinds of activities are time-consuming and we want them to have been carried out as soon as possible, if not sooner."

Infotheek in Leiden and Groenendijk in Ridderkerk also illustrate the opportunities of the Randstad production environment with respect to producer services. When director Van Leenen of the Infotheek Group cannot find an eligible marketing bureau in Leiden itself - "No good advertising agencies are locnted in Leiden" - he turns to a firm 
in Rotterdam, which is only $36 \mathrm{~km}$ from Leiden, and to a firm in Amsterdam $(38 \mathrm{~km})$. Furthermore, Van Leenen says: "If I could get an advertising agency from Leiden, I would prefer that, because now we have to incur a loss of travelling time. These are inefficiencies which I want to have as low as possible." Other supporting service firms also are provided from within the business area, such as accountancy (Arthur Andersen \& Co., The Hague, $15 \mathrm{~km}$ ), notary services (Amsterdam), and headhunters (Leiden).

Groenendijk plc in Ridderkerk initially made use of accountancy and notary services from Amsterdam $(73 \mathrm{~km})$. Groenendijk: "This occurred because of personal relationships. Some yenrs ago, we reversed that for practical reasons. We have switched to businesses in Rotterdam [which is adjacent to Ridderkerk]. When one has to visit the notary or the accountant [Coopers \& Lijbrand Dijker van Dien] it is ensier to travel to Rotterdam than to Amsterdam. For example, when we applied for a WIRpremium, we called in the advice of the notary per order. It was very pleasant that it was possible for him to come around to our office every time again."

Waterland Automation also obtains the majority of the external business services the firm needs from the regional production environment. Director Schoneveld: "As far as services are concenied we rely on Amsterdam [15 km]." However, some services do not come from Amsterdam, although still from within the regional production environment. Auditing is performed by a chartered accountant, Paardekoper \& Hofman, in Zaandam (13 km), for example. Furthermore, Waterland has called in the help of Digital Holland in Utrecht $(52 \mathrm{~km})$ for the implementation of an office automation system in order to be able to do its administration in-house. Two important services are located outside of the business region. These are Pandata in Rijswijk $(72 \mathrm{~km})$, for training of employees, and IDC in Brussels $(225 \mathrm{~km})$, for international market research.

\subsubsection{Summary}

These case studies allow us to draw two major conclusions with respect to the interaction between the firm and its (service) environment. First, it appears that nearly all firms in the survey, both Randstad and non-Randstad (perhaps with the exception of Elephant Industries in Hoorn), are able to make fairly intensive use of the local region for external service functions. Secondly, this does not detract from the fact that peripheral area firms in the sample, as they develop, increasingly have to take a proactive stance toward the local services infrastructure. Of the businesses investigated, this expresses itself most clearly in non-Randstad firms purchasing service inputs from outside the local area, particularly from the Randstad. Randstad firms, in contrast, can continue to rely on their business region, if not their place of location, as they grow.

About internalization or non-use of service functions, as other types of responses to inadequate external supply, no definite statements can be made on basis of the firms investigated, since such behaviour is often not directly related to conditions in the local environment. On the other hand, particularly because of inadequate external supply, self-service unmistakably occurs. It has been recorded thoroughly, albeit not in response to ineligible local supply, but instead of inconvenient non-local, i.e. extra- 
regional, support. On the face of it, this suggests that decision-makers first seek to satisfy external service functions within the business region. If this fails (or actors think it will), they try to find the services outside of the business region. Only after this also proves unsatisfactory (or actors think it will) might they start internalizing (or keep on switching and searching, of course).

Anyhow, the sample firms reveal a major flaw in the prevailing theories about the role of service functions in the environment. It is maintained that spatial differences in the availability of specialized and high-quality services are an important factor in creating inter-regional differences in business competitiveness, and furthermore it is implied that this relation is a rigid one. This line of reasoning fails to take into account the fact that relations between firm and service environment work out in a more dynamic way, along with the growth stage of the firm. From the directors' accounts it can be inferred that a number of service functions - headhunters, market research bureaus, and all sorts of advice which accompany events such as quotation on the stock exchange, take-over and acquisition activities, restructuring measures and the like - are used only after the firm has been in operation for several years and when it has achieved considerable size. Furthermore, in their early growth stages, firms not only use a smaller number of service functions, but they also call in less prominent and less professional supplier firms than later on. For example, it is very unlikely that the reputable nationally and internationally operating accountancy bureaus which provide their services to the sample firms have been called in from the outset. In their early growth stages, businesses simply cannot afford such a luxury. The same phenomenon has been observed with respect to hiring labour (cf Section 5.2). Calling in McKinsey to consult a small firm of ten, 20 or 30 employees is like cracking a nut with a sledgehammer. In sum, firms that are young and small are far less demanding with respect to the local supporting service infrastructure than larger ones. This implies that for their early development they need not be located in a region that is optimally endowed with all sorts of specialized, high-quality services. By the time they do require this kind of service input, the firms have grown larger and their capacity to deal with local deficiencies has increased. This is more or less admitted by O'Farrell and Hitchens, when they write that "Insofar as larger enterprises are able to internalize many service inputs, the location and local availability of external suppliers may not be important" (O'Farrell and Hitchens 1990, 1147). The impact of the extra costs attached to resistance strategies in response to local inadequacies gradually diminishes as the firm increases in size. So the interviews point towards a mutually reinforcing, dynamic interaction between on the one hand organizational size and adaptability, and, on the other hand environmental pressures, as has been outlined in Chapter three, Section 3.4. and Figure 3.5.

\subsection{Meeting the market environment}

Up to now, this chapter has devoted attention to input resources; the present section focusses on output resources. It is widely assumed that the growth of enterprises will be accompanied by a progressive expansion of the geographical range of its activities, like ripples spreading in a pond. This means that firms' activities develop from local 
or regional to national or international spheres of operation. In the early life of a firm, sales are heavily concentrated within the local market. When the local market is saturated, the firm expands to the regional scale by setting up sales offices. In the subsequent stage, activities are extended to the national and eventually to the international scale, either by acquisition of other establishments or by setting up capacity of its own. Models of corporate growth have been developed by Taylor (1975), Watts (1980) and Hakanson (1979). In all these models a firm's access to a large and growing urban market by virtue of its location is of vital importance for triggering growth of the firm. According to Haikanson (1979, 132), "During the early life of a firm, its ties with the immediate surrounding environment are likely to be very close. Personal contacts with financiers, suppliers, and customers are often decisive factors for early survival and growth." Small, new firms in peripheral areas, in contrast, fall into a vicious circle of constraint. Because the local and regional markets prove too small to sustain production at the levels required for profitable operations, the need to break out of the local area and to operate on a supraregional market emerges soon after start-up. However, McDermott and Taylor $(1982,62)$ assert that it is no sinecure for small firms to achieve this. According to them, only larger firms would be able to extend their action space beyond the regional level. It is assumed that small firms face formidable marketing problems, because they lack both the expertise and sufficient financial means to sell their products on wider geographical markets (cf for example Hall 1985, 53). According to McDermott (1974), entrepreneurs find it easier to monopolize the local market than to compete in supraregional ones (spatial monopoly hypothesis).

The dependence of new firms on the local market as a launch-pad for start-up is recognized in many new business surveys. For example, Dutch research conducted among very young companies of at most three to four years old shows that sales of 72.6 per cent of these businesses do not go beyond the regional scale (VNO/AMRO 1988, Table 14). De Jong (1987) investigated firms which were somewhat older, i.e. at most ten years. This research, which was conducted among businesses in the northern wing of the Randstad (i.e. the cities of Haarlem, Amsterdam and Utrecht and the surrounding areas) suggests that slightly more older businesses are still very strongly attached to the regional market. No less than 83 per cent of the surveyed firms booked over 50 per cent of their sales in the westem part of the country. Similar results are found by Lloyd and Dicken (1982) in the British conurbations of Manchester and Merseyside. More than half of all the sampled new firms, which were less than five years old, contracted in excess of 50 percent of their business in the local conurbation, and one quarter found 90 percent of their sales within the immediate urban area. This market context differs minimally for firms between five and ten years old. Indeed, the latter category reveals an even closer attachment to the immediate conurbation market, with one-third depending on local sources for more than 90 per cent of their business.

Well, how do the survey firms encounter the market. Particularly, how do they respond to hindrances and opportunities? 


\subsubsection{Manipulation}

\section{Enticing and leverage}

To begin with, I will demonstrate that a firm's clientele is not unilaterally dependent on the presence of that firm in the local business area. This subsection highlights the influence which the sample firms exert on the market. The businesses investigated employ several methods to influence the disposition of the market in favour of their own survival. Many incentives can be detected which either deliberately or inadvertently entice potential customers to buy a company's goods or services. Working the market occurs deliberately by employing such well-known measures as advertising in trade journals, conducting direct mailings, preparing prestigious annual reports, taking part in trade fairs, dispatching internal salesmen and/or external sales reps. But also utilising in reputable material and service input suppliers is meant to foster goodwill in the market. Three sample firms have undertaken some more unusual initiatives in this respect. System House Comdes, for example, under the regime of Quekel and Kragtwijk, organized rond shows and also an annual tennis tournament in 's-Hertogenbosch (in cooperation with Nashua Holland). When Comdes was in serious trouble and was taken over by the Modalfa/Mobeta investment group, the new director, Mr. Vissers, worked hard to restore the image of the company in the market. So, Vissers organized symposia and meetings in which well-known people take part. Vissers gave an example: "Yesterday evening I held a conversation with Professor Nieuwenburg, who seems willing to present a lecture at a symposium we are organizing. This symposium is meant for corporate investors, financial organizations and insurance companies. The organization of this symposium occurs against the background of a software package called 'Rendement', which we designed for controlling investment portfolios. Nieuwenburg is prepared to discuss our product and to talk about the market for corporate investments. The director of Bogamij, Mister Blnisius, will act as a discussant for the paper of Nieuwenburg. In this way, by inviting and establishing relations with important players on the market for investments and by subsequently attempting to give follow-up to these connections afterwards, we try to restore our image."

Furthermore, in its heyday Comdes published an irregularly appearing news magazine about its advances in the sphere of software engineering, to which one can subscribe. Database Consultancy International does the same. Intercai's sponsorship of a well-known ice hockey team in the Netherlands, the Smoke Eaters, is yet another example of working the market.

A medium which is becoming increasingly important for small and medium-sized manufacturing firms as a marketing instrument is the successful introduction of the so-called ISO 9001 Quality Management System into the firm. An ISO 9001 certificate represents the official quality approval, not only of the firm's products but of the entire organization. To obtain such certification, firms have to meet rigorous quality standards and to carry out radical adaptations. Such a certificate is seen as an important credential by (potential) customers. Several of the sample firms have either already obtained an ISO certificate or are working on it: Elephant Industries, Bronkhorst High Tech, Casolith Sheets Nedap and several Aalberts subsidiairies.

Enticing potential customers to buy the business's goods and services occurs not only by deliberate marketing. It also takes place, and maybe even more importantly, 
in an indirect, informal and less conscious way. A number of directors in the sample indicate that by far the most effective way to advertise the firm is through satisfied clients. Contented customers work as gateways to new ones. The directors of System House Comdes, Intercai, Waterland Automation, Hulo International, and Database Consultancy International indicate that word-of-mouth advertising is a major method for bringing in customers, if not the most important one. Some entrepreneurs actively exploit this fact by presenting contented customers in an eyecatching way in their annual report or advertising brochures and printing their praise of the goods or services delivered. In a similar vein, other beneficial occurrences in a firm's life cycle are exploited as a lever to influence the market. For example, prizes awarded, visits to the firm by well-known people such as leading politicians and members of the Royal Family, or quotation on the stock exchange are deliberately used as marketing instruments.

In their heyday, the automation companies Infotheek, System House Comdes, and Waterland Automation 'used' a very strong lever to gain access to the market, namely their primary computer hardware suppliers, respectively IBM, Wang, and Digital. The three automation firms were able to take significant advantage of their relations and exploit the sales outlets and brand names of the latter.

It must be added, however, that many of such marketing activities are more likely to follow firm growth than cause it. They come into existence only when the company is experiencing substantial growth; they vanish into thin air as soon as hard times are coming. They fall into the category of what Storper and Walker (1989, 44-45) call 'weak competition', i.e. jostling for improvement of a firm's market position but not determining it.

\subsubsection{Immunization}

\section{Integration}

Apart from attempting to influence the behaviour of actual and potential customers, the surveyed businesses also take measures to defend themselves against certain market barriers or deficiencies. One such group of measures can be subsumed under the heading of integration. For example, in order to improve its grip on the market, Aalberts Industries has moved increasingly from producing input supplies towards manufacturing final products. To that end, Aalberts Industries has taken over medium-sized customer firms (200 - 300 employees) which produce final products on the basis of non-ferrous input materials, such as water and gas pipes, and beer appendages. Director Aalberts: "We increasingly realized that we had no good hold on our markets. As an industrial supplier firm, one's fortunes are very dependent on the success of one's customers." This changes when a firm produces the end products itself (Het Financieele Dagblad 4-9-1991).

In the Netherlands and Belgium, Groenendijk ple has extended its geographical market outreach by establishing its own subsidiary plants, while in Germany the firm has expanded by taking over a German plant in the same branch. The main reason for pursuing a different geographical expansion strategy in Germany than Netherlands and Belgium is to hedge against restraints imposed by custom barriers or by 
unfamiliar market conditions in Germany. Director Groenendijk: "In Belgium people are familiar with Dutch products. In Germnny this is different; over there one has to see to a strong domestic identity of the goods or seroices delivered. I think in Germany it is more difficult to establish subsidiaries from the ground up than it was in Belgium." This very same internationalization strategy is applied by Intercai. Whereas in Belgium the company has established a subsidiary by itself, expansion in Germany, Switzerland and Italy has occured through taking over foreign telecommunication consultancy firms.

\section{Scattering}

A second group of measures which shield the firms from the contingencies of separate segments of the market environment includes the scattering of market relations by means of diversification strategies. So, no single company in the sample has its fortunes tied to one or a few large customers or market segments. For example, the most important market segment of Aalberts Industries, aircraft construction (e.g. flight recorders for Fokker and Airbus), accounts for only eight per cent of total sales, and no other customer is worth over three per cent of its trade. These are iron laws within the company (FEM 25-2-1989).

Nedap develops electronic identification systems for the retail trade (antishoplifting systems), for premises (hands-free access control systems), for cattlebreeding (automatic cattle management systems), manufacturing industry (e.g. automatic material handling systems, tool identification systems), and for many more markets.

Casolith Sheets adapts its synthetic sheets for use as an input material in the illuminated advertising industry, in the construction sector (noise barriers, balcony fences etc.), and in the sanitary industry. System House Comdes develops software packages for hospital automation, corporate investors, and the printing industry. Intercai has attuned its telematics consultancy services to the requirements of several different market segments, such as national government, health care, agro-business, tourism, transport, industry, manufacturing industry and the financial and insurance sector.

Director Brouwers of Elephant Industries explicitly pursues a diversification policy with respect to geographical markets (geographical market development). Brouwers: "Intentionally, we do not want to develop a large market share in one particular country. Instead we prefer a small portion in many nations. This is our philosophy. In this way we are not dependent on one, two or three markets." Many more examples of diversification by means of market development could be added here if we could spare the space.

Diversification occurs not only with respect to markets but also with respect to products. So, Intercai's initial consultancy activities consist of supervising and managing the technical installation of new communication systems (cable television mains, computer networks, air networks, company switchboards). However, in the course of time, Intercai has also become involved in other parts of the whole process of introducing new communication systems to organizations fanning out from its niche of consulting about the technical installation. The firm has also started commissioning consultancy activities concerning basic policy lines in organizations with respect to new communication systems (these activities occur prior to the actual technical implementation of the installation of these systems), their organizational 
implications, and the education of staff workers to handle the new systems, as well as their maintenance. Staff worker Donders: "When initial consultancy about one fragment of the entire process is good, organizations call you in for consultancy on other parts as well." These so-called turn-key services, which constitute an incidence of a vertical differentiation strategy, are not unique to Intercai. They are typical for several other service firms in the sample, such as Rood Testhouse, Waterland Automation, System House Comdes, and Infotheek. Vertical differentiation is mainly presented as a growth strategy (Ansoff 1987), but at the same time it hedges businesses against drawbacks in separate products or types of business activity. The Dutch consultancy market for building local cable television mains is rapidly becoming saturated since the majority of the local communities in the Netherlands has already been provided with cable television. Nonetheless, Intercai's consultancy activities regarding cable television mains are not diminishing. Donders: "This is because at the level of local governments reorganizations are taking place which require linking of distinct cable television mains. There is also a rising need to replace or modernize obsolete mains."

\section{Rationing}

A third cluster of measures which immunize against segments of the market environment involves rationing market relations. Rationing strategies are used, for example, by start-up companies to by-pass entry barriers. Remarkable instances of this strategy can be found in the start-up phases of Aalberts Industries and Bronkhorst High Tech. Recently Aalberts Industries, which predominantly produces supplies for niche markets, is increasingly producing for final markets. Director Aalberts admits that he projected this already at the start-up of the company. Aalberts: "When I started in 1975 I thought: I want to produce final products using my own technology. But I did not realize then that selling them would cost so much money. Production I would be able to afford, marketing definitely not" (FEM 13-6-1992). After a difficult start-up period, Aalberts decided (for the time being) to avoid the barrier of addressing final markets and to start as a supplier company.

When Bruggeman and Jouwsma started their firm for electronic gas flowmeters (Bronkhorst High Tech), they initially targetted the very big OEM market, i.e. Original Equipment Manufacturers, which encompasses the semiconductor industry, among others. OEM firms use gas flowmeters as components in their own machines. However, two major problems arise when addressing this market. In the first place OEM manufacturers are strongly inclined to use only well-established and familiar input materials in their (sub)assemblies. This is because the quality of their machines is directly related to the quality of their input devices. So, they are aversive to risktaking in this respect. A second problem attached to the OEM market, which director Jouwsma mentions, is that Bronkhorst's gas flowmeters are built into appliances which themselves are still being made. Jouwsma: "So their development period is added to the time lag. In practice you are not able to produce until one-and-a half to two years after the instrument has been offered for evaluntion. So as a company, you need a long lead time. We didn't have that when we were starting out." For these reasons, Bruggeman and Jouwsma decided to avoid the OEM market and to start their activities in a different market. True, that market is much more limited but at the same time it is also more 
accessible to a new firm. This is the chemical laboratory market. Because labs use the gas flowmeters of Bronkhorst High Tech as separate instruments they are more inclined to experiment with them and to evaluate their performance instantly. When Bronkhorst High Tech had established sufficient credibility and a reputation in the labs market the company was able to address the OEM market successfully. At the date of the interview, Bronkhorst was following this very same strategy to introduce electronic liquid flowmeters onto the market.

Rationing against elements of the market environment occurs not only in the initial but also in more mature stages of a firm's development, such as with restructuring processes and operations. For example, director Vissers of System House Comdes hived off computer trade activities when he reorganized the company. He wanted to be able to concentrate totally on software engineering and to make the company less directly susceptible to developments in the hardware trade. Similar measures have been taken by director Schoneveld of Waterland Automation when he restructured, rationalized and specialized Waterland Automation. When director Jansen started reorganizing Casolith Sheets in the early 1980s, he abandoned the production of casein, which is a synthetic material made from milk proteins. Manufacturing casein was the company's original core activity. Jansen: "That nearly broke my hart, because we had 50 per cent of the European market for that product. However, we needed 100 per cent to be able to gain something by it."

From the above instances of immunization strategies against either actual or perceived market hindrances, no difference between Randstad and non-Randstad businesses was discovered in the sample. However, one sharp difference between these two groups of firms did emerge. The more limited local market conditions in the periphery relative to conditions in the Randstad provoke non-Randstad businesses to skip the home region as a sales area and to develop an extra-regional market outreach very early in their life cycle, if not from the very beginning. At the time the interviews took place, out of the nine peripheral area firms in the sample, eight sold negligible amounts or nothing at all to the regional home base (Table 5.1), whereas Intercai's regional content was about 20 to 30 per cent of total sales.

So, at the time of the interviews, the non-Randstad businesses sold their goods and services on a national or an international scale, to a much larger extent than the Randstad firms in the sample. What is more, all but one of the (younger) peripheral area firms in the sample have to do without local customers from the very start of their businesses, as revealed by the directors' accounts. Take, for instance, the comment of director Bijsterbosch of Rood Testhouse: "Our starting point was the rise of micro-electronics. It was very difficult for user firms to come by test software, test programs, and everything that entails. Thus, we thought we had a good chance to perform a service function in that area. This was especinlly the case in Germany; there the clients demand quality. So actually, we started out on the German market." Or take the statement of Director Brouwers of Elephant Industries: "To us Germany has been a large market from the outset, larger than we would like it to be." Director Jouwsma of Bronkhorst High Tech: "We started with three sales reps; one in the Netherlands and two in Germany. At the same time this represented the geogrnphical distribution of sales: one-third in the Netherlands and two-thirds in Germany. This was how we started." Aalberts Industries had its major customers in the early development period mainly outside the local business region; 
these were Philips (Eindhoven, $55 \mathrm{~km}$ ), IBM (Amsterdam, $181 \mathrm{~km}$ ), Siemens (The Hague $189 \mathrm{~km}$ ). One important early customer - Oce van der Grinten - is located in the vicinity of the firm, namely in Venlo. System House Comdes also found its initial customers mainly outside of the home region - in Amsterdam (Lukas Hospital, Bank

Table 5.1 Spatial scope of the the sample firms' sales linkages in 1988

\begin{tabular}{lrrr}
\hline Firm & $\begin{array}{c}\text { Home } \\
\text { region }\end{array}$ & $\begin{array}{c}\text { Remaining } \\
\text { Netherlands }\end{array}$ & Abroad \\
\hline & Non-Randstad & & $\%$ \\
\hline Aalberts Industries & 2 & $50-60$ & $40-50$ \\
System House Comdes & 10 & 80 & 10 \\
Elephant Industries & 0 & 19 & 81 \\
Hulo International & 0 & 10 & 90 \\
Intercai & $20-30$ & $65-75$ & 5 \\
Casolith Sheets & 4 & 26 & 70 \\
Bronkhorst High Tech & 0 & 9 & 91 \\
Rood Testhouse & 0 & 30 & 70 \\
Nedap & 0 & 40 & 60 \\
\hline & Randstad & & 12 \\
\hline Database Consultancy Interna- & vast ma- & minority & 10 \\
tional & jority & 35 & 10 \\
Groenendijk & 55 & 20 & 12 \\
Infotheek & 70 & 23 & \\
Waterland Automation & 65 & & \\
\hline \hline
\end{tabular}

Mees \& Hope), and in Rotterdam - although two clients were located within the province of Brabant. Anyhow, director Kragtwijk complains about the local market domain: "The region of Brabant prefers being served by firms from the Randstad. Now and then I have a feeling that this is because Brabant people consider themselves [and by extension Brabant-grown products] a bit inferior to people from the Randstad." It is clear that none of the enterprises mentioned above could ever had got off the ground if they had been dependent on the local market as a launch pad.

There is, however, one peripheral area company in the sample which forms an exception to this picture. This is Intercai, which could take advantage of local customers as a launching site for a quite successful growth. However, very soon after 
start-up, the company broke through the boundaries of its home region And after being in existence for about four years, the majonty of sales were made in the Randstad

\section{Adaptation}

The above subsections give an impression of how firms attempt to work the market in their favour or to protect themselves against certain threatening elements of the market environment Yet it is beyond dispute that the growth of the surveyed firms has occurred essentially because they have been able to discover and to adapt to the needs and desires of the market Their adaptiveness to the market is the reason for the firms' basic competitive edge on competitors The only viable way for small firms to influence the customers' behaviour is by fulfilling their needs This does not mean that the sample firms fit in with the customers' requirements in a perfect way Rather, they provide, over a certain period, a more satisfactory solution to customers' needs than a whole range of alternative opportunities

The businesses investigated demonstrated that the adaptation process occurs on two levels, $1 \mathrm{e}$ a supplementary and a basic level. These two levels can be viewed in the context of distinction made by Storper and Walker between strong and weak competition respectively (Storper and Walker 1989). Sticking to the sample firms, it appears that supplementary adaptation occurs roughly according to the following pattern Entrepreneurs or firms master a basic technology or skill, which they are able to adapt to the requirements of different users This may occur in the form of standardized production in smaller or larger senes for separate industries or types of functions Otherwise, or at the same time, it may occur in the form of tailor-made production for individual customers By the way, standardized series of goods or services for particular lines of business may be further adapted to the requirements of individual users

Without doubt, the fine tuning of technologies or products to (groups of) customers is a market-led phenomenon However, what about basic skills or technology which constitute the basic strength of the business ( $\mathrm{e}$ strong competition), are they market or technology-dnven? Well, as far as I could gather, most firms in the survey also gleaned their central ideas from the market So, according to Jouwsma (Bronkhorst High Tech) his companion Bruggeman contacted him in March 1981 with a proposal to start a business of their own, saying "I have an idea to develop a product for which a good market exists " Director Groenendıjk recounted the gist idea which lies at the bottom of the important letting division of makeshift accommodation of Groenendijk plc "The firm was founded as a transport undertakng One of the activities was the transportation of construction sheds used by road crews, painters, construction workers and the like From the contacts with these people, at some point the idea emerged not only to transport but also to let out these construction sheds This actually meant the start of our letting activities, some 15 years ago " At the end of the 1970s, Mr Lightbody, director of an electronic and instruments engineering company, was toying with the idea of expanding his firm by adding a third division, which should undertake telecommunication consultancy activities Henk Kivits was willing to pick up the idea and work it 
out, though not as a part of Lightbody's business, but as an independent firm, called Intercai. Lightbody agreed with this. On the origin of Lightbody's idea for developing telecommunication consultancy activities, staff worker Donders says: "Some ten years ago Lightbody saw that electronic engineering and instrumentation activities would incrensingly be dealing with developments on the telecommunication market, and that the information technology market would come along in its wake."

Director Schoneveld's (Warterland Automation) idea to establish his own business in the sphere of administrative automation in 1978 was equally inspired by market opportunities. Schoneveld: "I was previously head of an automation department in the field of process automation in a manufacturing firm, VMF Stork in Amsterdam. From our basis in process automation, we increasingly had to deal with complete business automation. Against this background we conducted a fnir amount of research on mini-computer systems. They were picking up in those days and made it in principle possible for firms to undertake automation by themselves. Growing conflicts between firms and central computer centres, and the possibility to decentralize automation activities to individual firms, inspired me to start a business of my own to further develop administrative business automation."

Here it is useful to repeat Bijsterbosch's account of the start-up of Rood Testhouse: "Our starting point was the rise of micro-electronics. It was very difficult for user firms to come by test software, test programs, and everything that entails. Thus, we thought we had a good chantce to perform a service function in that area." Also the foundation of other sampled businesses, such as System House Comdes and Elephant Industries, was inspired by market considerations. Small wonder, since the initiative to set up independent firms was taken by entrepreneurs, respectively Quekel and Brouwers, with a commercial background, i.e. people with previous work experience in commercial functions.

Nedap, however, constitutes a clear exception to the above pattern of market-led innovations, since the company's basic technology, electronic identification systems, is strongly technology-driven (cf, for example, Paijens 1989). Anyhow, irrespective of whether the firm's know-how or skills have been pushed by technology or pulled by demand, their success in the market reflects primarily their adaptiveness to customers' needs and desires. It is out of the question that the businesses studied have either the power or the means to impose their products on the market.

\subsubsection{Utilization}

With respect to input resources, all sampled businesses, i.e. both those within and outside of the Randstad, are able to make significant use of local conditions. Yet with respect to output resources, things are quite different. Unlike their counterparts outside of the Randstad, Randstad firms, from the very beginning of their business activity up to the moment of the interviews, have been able to sell the bulk of their products and services to local customers (Table 5.1). The Randstad entrepreneurs consider it of great importance to be located in the vicinity of their customers in order to gain access to them. This is most clearly revealed in the directors' answers to the question whether they think their businesses would have achieved the same rate of growth had they been located elsewhere in the country. Spontaneously, the respon- 
dents name the proximity of customers as the decisive factor. Director Groenendijk of Groenendijk plc: "I think that here in Rotterdam the market is bigger than elsewhere. Even a location in the northern part of the Randstad would have made our growth rate less rapid, because quite a lot of makeshift sheds are let to industries here in the Rotterdam area, such as the petrochemical industry in the Europoort area."

The other Randstad directors think that location elasticity is slightly higher. Director Van Leenen (Infotheek): "Within the Randstad I would have achieved the current growth of the company in any location; outside of the Randstad not, however. In that case, 'distance' would emerge as the limiting factor. The majority of our clients are located in the Randstad. Since personal visits are important to our firm, one has to take travelling distnnce and travelling time into account. Well, in the case of a location outside of the Randstad, traffic jams would play an enormous, negative role. We establish sales outlets in the big cities; we have an outlet in Amsterdam [38 km] in order to be sited near our custonters." Others express similar viewpoints. According to director De Zoete of Database Consultancy International: "A location in the North of the Netherlands, for example, would be very unfortunate, because our clients are big companies, of which the vast majority is located in the Randstad." And in the words of director Schoneveld of Waterlaud Automation: "I think that it is all the same whether we approach larger internationally operating companies from Purmerend or from elsewhere. But then I am referring specifically to the Randstad."

The only non-Randstad business in the sample to which local customers have been important is Intercai. The company was launched by two important orders for consultancy services concerning the installation of cable television mains. A third order from the provincial authority of the Province of Limburg in Maastricht $(15 \mathrm{~km})$ in 1983 was the break through for Intercai as far as director Kivits is concerned. To the question whether he thinks that Intercai would have achieved the same success in other places in the Netherlands, Kivits answers: "If local and provincial authorities in other places would have been as cooperative as those here in Limburg, I think that it would have occurred in Groningen, Leeuwarden, Appingendam, Delfzijl, or wherever, in the same way as it has at the present location. But local authorities have been very valuable to us, since they did give us the opportunity to do our first projects. I am very grateful to them." However, now about half of the company's clients are located in the Randstad, at a distance of over $170 \mathrm{~km}$ from Geleen. Kivits thinks that this disadvantage is compensated for by the image of the Province of Limburg as a relatively small-scale community and as a region centrally placed in Europe. For these reasons, Kivits thinks that "Intercai as a consultancy bureau is better able to proliferate itself from Limburg than from the Randstad." Anyhow, it is salient that Intercai did not establish sales outlets in the Randstad nor in any other region of the country. Staff worker Donders emphasizes that the subsidiaries which Intercai did found in the Randstad and in other parts of the country do not function as sales outlets. Intercai's affiliates are functionally separated, and every single subsidiary covers the national market for its particular function(s). So, the Geleen office is specialized in the market segments of health care, media and regional policy. The Rotterdam office, on the other hand, is specialized in the segments of finance, insurance, and water transportation. In the Utrecht office, the unit of air, road and rail transport has been concentrated, as well as the units of tourism, agro-business, and national government. And Intercai Veldhoven, near Eindhoven, concentrates on manufacturing industry. 


\subsubsection{Summary}

It appears that the firms investigated here deal with inadequacies in the market in many different ways, of which adaptation is the most significant. In encountering the market, there is one notable difference between Randstad firms and non-Randstad firms in the sample, which also has been observed with respect to input linkages. This is that limited sales opportunities in the home region have induced the non-Randstad firms to pursue more intensively the adoption of a wider geographical action space than the Randstad-based companies. However, local market conditions in the periphery are nevertheless distinctive from local input conditions. The former (markets) are restricted to a much greater extent than the latter (inputs). Hence, local market conditions call forth more drastic counteraction than local input conditions. So, the more restricted availability of input supplies in peripheral areas does not become manifest to non-Randstad firms until they have reached a certain size and have been in existence for several years. In contrast, peripheral demand conditions are such that firms often have to make do without regional customers from the very beginning. Chapter Seven will elaborate on how these firms have succeeded in getting started while lacking any local output resources. Chapter Six attempts to explain why this situation has never undermined or hampered their growth.

\subsection{Coping with the environment; summary and conclusions}

This chapter has attempted to give some insight from a shop-floor perspective into the extent to which small and medium-sized businesses can take advantage of external resources. It also examined how these firms respond to actual and/or potential inconveniences in utilizing these resources. The recapitulation of this will take place in two steps. First, small-firm conduct towards external conditions will be summarized from a spatial point of view. Secondly, some concluding remarks will be made concerning the framework used to categorize firms' attempts to cope with external resource contingencies in general.

\subsubsection{The spatial dimension}

From a spatial perspective, three observations on small-firm behaviour towards the environment can be made on the basis of the empirical material presented in this chapter. One is that both Randstad and non-Randstad firms are able to take considerable advantage of local conditions. Centre-periphery contrasts seem to be moderate in the Netherlands. Secondly, the foregoing contention should be immediately qualified. On the one hand, convergence of economic conditions across space is related to the growth stage of a firm. As firms mature and professionalize, they raise their expectations of the quantity as well as the quality of external inputs and deliveries. Ultimately, greater resource capacities of central areas may become manifest. On the other hand, inter-regional convergence of economic conditions is different with respect to type of resources. Centre-periphery contrasts are greater and more discriminating in 
regard to local sales opportunities than to input resources. The third observation is that these comparative disadvantages did not prevent the peripheral firms from being successful. They are in principle able to overcome the constraints of the production environment by manipulating external resource conditions, by immunizing against them, or by adapting to them. In practice, coping with centre-periphery contrasts concerning the availability of resources manifests itself most clearly in non-Randstad businesses that had adopted a much wider geographical action radius earlier in their development than Randstad firms. I have interpreted this as a form of immunizing against local resource scarcities. With respect to manipulation, internalization, or adaptation strategies, no clear spatial dimension can be determined from the sample firms. It did not prove that non-Randstad firms took recourse to such strategies more frequently and intensively than Randstad firms. It is possible however, that a larger research sample would have revealed this as well, though perhaps somewhat less markedly.

\subsubsection{A general picture}

In the present chapter, attention has been focussed on action and counteraction. It must be noted that firms do not or are not able to react to every environmental threat or barrier they encounter. It also occurs that entrepreneurs go into action too late, not rigorously enough, or otherwise act in an inadequate way. This chapter is not meant to depict the survey firms as being able to resolve all the problems they run into, certainly not. The intention is to highlight the point that small firms need not be completely at the mercy of their environment, as is implied by ecological perspectives in both regional and administrative science. Surprisingly, many ways and means can be used by small firms to mitigate local resource deficiencies. This does not occur according to grandly designed strategic plans aimed at autonomous goal achievement, as is suggested by the bulk of corporate geography literature in regional science and by the rational action approach in administrative science. Rather, firms tackle the environment by means of numerous measures of all kinds. These vary from high-cost to low-cost, from permanent to temporary, from locational to in situ, from conspicuous to inconspicuous, from drastic to moderate, and so forth and so on.

In general, the sample businesses give the image of being involved in an endless struggle to bring firm and environment in line with each other. In other words, a firm continually tries to satisfy its resource needs, not so much through selective, sophisticated strategies but rather via trial and error and taking many different, ad hoc measures simultaneously. On the other hand, the firms do not have to fight for every single resource unit required. Through prestige and reputation, firms exert influence on elements of the environment. These are the spill-over effects of prior successful growth. Such effects have been found to operate in regard to acquiring labour, financing, suppliers and customers.

At the time, the businesses studied frequently have to adjust their objectives and change their tack in response to environmental constraints in order to survive. Sometimes, at a later point in time, initial objectives are eventually realized, either expectedly or surprisingly. Other adjustments, in contrast, cannot be reconsidered 
anymore. They will exert their influence for years to come, such as when autonomy is lost. In this way, the survey firms are undisputedly affected by the environment in their heyday. However, they are not determined by it.

The organizations' responses to the environment have been arranged according to how they deal with perceived or actual environmental constraints and opportunities. As a result, four types of action have been distinguished: manipulation, immunization, adaptation, and utilization. At this point it should be reiterated that although the firms' actions have been presented as discrete strategies having clear-cut effects, these methods and effects are inextricably intertwined. There are many possible combinations of the foregoing perspectives that can characterize the interaction between a formal organization and its relevant environment. For example, before firms are able to influence customers' behaviour they first have to adapt to the buyers' needs and desires. Or, in order to fulfil the requirements of one actor in the environment, firms may have to exert pressure on others. So it is worth noting that the characteristics of small-firm conduct towards the environment may function as both means and ends (see also Chapter Three, note 3 ). Because of this interwoven aspect it is sometimes difficult adequately to classify actions into one of the four categories used. Clearly, each of these four perspectives represents only a partial view of the complex reality of small-firm behaviour towards the relevant environment. Only together do they provide a useful repertoire of complementary ways to deal with external threats and opportunities.

Reviewing how small firms manage and resist the environment in which they are located, the rigorous environmentalist may object that ecological views do not contend that firms remain inactive vis-à-vis environmental conditions; rather, they are not able to overcome disadvantages effectively (see also Chapter Two, Section 2.3.3). In other words, even if organizations act against their environment energetically, all their attempts and endeavours can have only a symbolic value. This matter will be dealt with in the next chapter.

\section{Notes}

1. Donders is not dissatisfied with the labour supply in the local business region. Nonetheless, he acknowledges that acquisition of new personnel is easier in the Randstad than in Limburg. Donders: "Why is acquisition of new consultants more difficult in Limburg than in the Randstad? Because the entire market stmucture is concentrated in the westem part of the country. When one realizes that it is there where the key firms in the field of telecommunications, such as Alcatel and PTT, are located, it stands to reason that it is easier to attract new personnel there than here in Gelecn",

2. Kragtwijk gives two reasons for the organization's orientation toward the regional labour pool: "In the first place, the wage level is lower in Brabant than in the Randstad. Secondly, if we succeed in making people from Brabant feel at ease in our company, and when they have the opportunity to show their abilities, they will be less inclined to leave the finn in response to a higher wage offer elsewhere compared with employees living in the Randstad." Kragtwijk thinks that the farther people have to travel from home to work, the less loyal they will be to their employer and the more likely they will stay only for a short while at their current workplace. 
3. Elephant Industries constitutes an exception to this, since Brouwers received start-up capital from an American supplier firm. 


\section{Environmental responsiveness of small firms (II): its strength}

\subsection{Introduction}

The previous chapter described extensively how small to medium-sized firms respond to pressures from the environment, either by means of elaborate, intelligent strategies or through straightforward, everyday behaviour. An attempt was made to place entrepreneurial actions and reactions of all kinds in a spatial perspective. Of course, it remains to be seen whether this entrepreneurial jostling vis-à-vis the environment is really effective. Or is it little more than a futile show of opposition to an inescapable fate? This chapter devotes attention to the forces which can make a firm effectively overcome comparative disadvantages (i.e., research question number two).

Therefore we have to distance ourselves from the sample firms and resort to theoretical thinking. The framework for explaining the efficacy of resisting comparative disadvantages is basically drawn from the literature and from theoretical work in several closely related fields. Where possible, this picture will be documented by empirical evidence from the sample firms. All in all, this means that the explanatory framework cannot be supported by sufficient quantitative or qualitative information to be able to accept or reject it with certainty. The scheme draws together four themes in contemporary economic-industrial research: the growing importance of differential advantages in creating competitiveness and the concurrent demise of the role of comparative advantages; the dynamism and instability of competitiveness; first-mover advantages; and organizational learning.

It should be emphasized that organizational action is a prerequisite for mitigating locational or environmental disadvantages. Allowing mismatches between organization and environment to persist means that organizations eat into their slack (i.e., excess resources or reserves). Especially small organizations, which usually have little slack, cannot afford to let discrepancies between firm and environment exist without harming their market position. For instance, if the diminishing supply of appropriately skilled workers negatively affects the product quality of certain enterprises in a country or region, that downgrading will more rapidly and more adversely hamper the market position of a youthful small enterprise than that of a well-known established company. Whereas the reputation of the latter may form a temporary buffer against the effects of certain external threats, the former does not have that slack and must counteract the threats immediately. 
On the other hand, employing adjustment strategies does not necessarily imply that the adaptation is effective. Let us recall Figure 2.2, which posed the hypothetical case of two initially identical firms differing only in their location. One business lies in a well-provided-for region and enjoys comparative factor advantages. The other is sited in a poorly equipped peripheral region and faces comparative disadvantages. These territorial differences in the availability of resources manifest themselves in higher productivity of the favourably located, central area business relative to its remote contender (i.e. locational efficiency). In order to effectively overcome these locational disadvantages, catch-up strategies of the disadvantaged firm must fulfil three conditions. First, they must yield revenues in excess of costs. Secondly, their net result must be large enough to outweigh, or at least offset, the comparative disadvantage vis-à-vis the favourably located counterpart. And thirdly, this catch-up must be resistant to being nullified by means of simple imitation behaviour on the part of the central area firm. The next sections will deal consecutively with the costs, the revenues, and the sustainability of adaptation measures to environmental constraints.

\subsection{Adaptation costs}

Counteracting local resource scarcities has a cost. For example, supplementary inservice training of newly hired workers involves not only direct education costs but also important indirect costs of lost working hours. Compensating for labour market deficiencies by calling in headhunters to search for professional workers and hiring them away from other firms far and wide also bears extra costs. These consist, for example, of the fees of the headhunter bureaus called in; extra bonuses and other expenditures (e.g. moving expenses) to induce workers to leave their current place of residence and make a long-distance relocation; and the opportunity costs of a longer search time to find the appropriate workers. Or take another example: the extra high call-out charges or the opportunity costs of the accessory search and travelling time entrepreneurs have to incur when they must consult distant agents every time they need to call in external advice; or the extra costs of calling in either external sales reps or hiring separate salesmen to work distant markets when local market capacity is depleted or non-existent; or the greater need for distinct market research. Still another example concerns the extra costs attached to setting up internal supply capacity when this cannot be found in the vicinity; and consider the accompanying loss of flexibility and quality of inputs, to which flexible specialization theory devotes so much attention. Many more instances of extra costs attached to production in peripheral places vis-à-vis central areas could be mentioned, including partial or integral firm relocation.

However, not all adaptation behaviour is equally costly; some forms are clearly less expensive than others. For example, firms such as System House Comdes and Intercai, which provide training courses for the external labour market in their respective lines of business, take advantage of this activity by training and refreshing the skills of their own personnel without incurring any costs. Such spill-over effects are not restricted to the field of education and training, but may also take place in other domains. Intercai, for example, as a form of product development, supplies 
organizational advice concerning the implementation of new communication systems in organizations. The company also uses this know-how for the benefit of its own reorganization. This can be concluded from the response of the staff member Donders to the question why Intercai did not call in external consultants to guide the reorganization the firm was going through at the end of the 1980s. Donders: "If you can give outside advice, you should be able to give inside advice as well. We did not obtain external advice, apart from some legal counsel about statutory labour relations and some fiscal advice."

It also occurs that entrepreneurs receive external support to fill a gap in the production environment. For example, three firms in the sample received financial support from Local Employment Exchanges in providing training courses to the external labour force, as a way to upgrade the skills and know-how of the local labour supply (see Section 5.2). Other employers made use of cheap students on work placments, tailored their skills to the requirements of the respective production processes and subsequently attempted to incorporate them into their firm as soon as they finished their study and entered the labour market (section 5.2). In these cases the employers had a free ride on the budget of the Ministry of Education. Bronkhorst High Tech, another example, lets its personnel take computer courses which are financed by the branch organization the company is a member of. In passing, it should be kept in mind that these examples mainly concern savings in monetary terms, and that many costs remain. Take, for example, the opportunity costs of internal training and advice, i.e productive labour capacity being sacrificed.

However, sometimes adaptation is even virtually free. This is the case when adaptation results from a favourable reputation or image of a firm. For instance, the diminishing response from the local labour force to new job vacancies of Intercai is counterbalanced by an autonomous increase in the response from outside of the local business area. Equally, Elephant Industries is able to attract the great majority of its workers from a distance of at least $30 \mathrm{~km}$ without offering extra financial bonuses. Similarly, Intercai's extension of its geographical market area within the Netherlands, beyond the boundaries of the business region is achieved virtually without cost, since this is an autonomous process based on word-of-mouth advertising. Donders: "Territorial market expansion has never been a major goal of Intercai. We never said: now, we serve the Limburg market and within two years we want to supply the Brabant and Zeeland markets, and within five years the Randstad... In the field of cable television you have a government network to deal with.As long as you are able to supply a good product local governments recommend you in this circuit. Well then, it is a matter of leapfrogging from one local district to another. And from local authorities, linkages develop, both with Provincial authorities and with private businesses."

Furthermore, in a small country like the Netherlands, which is equipped with a very sophisticated road and communication infrastructure as well, distance costs are presumably modest. Thus, the extra costs of being located in non-central areas will be moderated according to urban field theory (cf Wever 1985). On the other hand, in spite of its elaborate road infrastructure, traffic congestion is an urgent economic problem. It jeopardizes the increased access of outlying firms to central facilities. Anyhow, the contention that in the Netherlands production cost differentials between regions can be ignored is in need of direct empirical verification. Up to now this has 
hardly occurred, mainly because of great methodological difficulties in measuring production cost differences across space. Nevertheless, such efforts would be most welcome to the field of regional science.

But even if production and adaptation costs in outer areas, within limits, do exceed costs in central locations, this does not rule out competitive production in such places (provided factorial resource endowment is not below a certain prohibitive threshold). One also has to incorporate the revenues of adaptation behaviour into the equation.

\subsection{Adaptation returns}

It is pointless to emphasize the costs of adaptation behaviour while neglecting its revenues. In modern trade theory, comparative advantages - that is, relative costs savings of production because of favourable local factor mixes - are increasingly pushed to the background as an explanation of trade patterns and competitive advantage. Endowed comparative advantages are increasingly being replaced by created absolute advantages as an explanation for trade patterns and competitive advantage. Thus, first it is argued that we now live in a world where factors of production for technologically stable products are not endowed, but instead "...created through investments made by individuals seeking to develop their skills, firms seeking the tools necessary for competing, and social institutions or governments hoping to benefit society or the economy" (Porter 1990a, 80). Almost any developed country making the effort can become as efficient as the next country in a technologically stable manufacturing sector. To buttress this contention, Storper (1992) refers to Amendola, Guerrieri and Padoan (1991), who show that technological variation among major countries tends to diminish steadily after major technological revolutions. An important force which diminishes the role of factor comparative advantages is technological change. As Porter (1990a, 14) states: "Technology has given firms the power to circumvent scarce factors via new products and processes. It has nullified, or reduced, the importance of certain factors of production that once loomed large." Porter gives the example of Swedish iron ore. The low phosphorous content of Swedish iron ores was an important advantage as long as the technology of steelmaking had difficulty dealing with impurities. As steelmaking improved, however, the phosphorous problem was solved, nullifying Sweden's factor advantage. Diminishing the importance of factor advantages not only occurs with respect to 'traditional' natural resources but also regarding 'modern' agglomeration advantages, such as labour. Ongoing automation and robotization is reducing the labour content of products in some industries, not only of low wage labour but increasingly also of medium and highly skilled workers.

Rapid interfirm and intercountry technological imitation behaviour makes such modem process and production techniques widely available. The result is convergence in the costs of production of different countries in a wide array of markets. Yet, trade and trade specialization between countries and regions persist and are increasing. So, as a second argument it is reasoned that something must make advantage possible in the face of rapid imitation by (potential) competitors. Many analysts now 
suggest that this force is continuous innovation on the basis of technological mastery, which gives rise to absolute advantages. The latter differ from comparative advantages: they reflect productivity gaps between countries or regions that are so great that they are insensitive to almost any set of changes in input prices (Dosi, Pavitt and Soete 1990; Storper 1992, 65). As such, the concept of absolute advantages is strongly related to the concept of differentintion, which incorporates the phenomenon of a firm creating a unique benefit that outweighs the disadvantages due to higher production costs (Porter 1985).

It is this notion of created absolute or differential advantages which underlies the explanation of observed disruptions of established positions of nations, regions and businesses, pushing the capitalist economy to grow and keeping it from ever settling into (neoclassical) equilibrium. Disadvantages and falling behind play a crucial role in this everlasting process of leaders being outwitted by laggards. According to Porter (1990a), innovation and advancement results from offsetting weaknesses, from being challenged by bottlenecks and threats, rather than from exploiting strengths. Disadvantages prod or force a nation's firms to seek new solutions. Thus, stress instead of munificence is the moving spirit behind innovation and advancement. As Porter states (1990a, 174): "Competitive advantage emerges from pressure, challenge, and adversity, rarely from an easy life'." Furthermore, Porter stresses that innovation to circumvent (selective) factor disadvantages is disruptive. This means that innovations may generate all kinds of benefits, though sometimes hard to anticipate, which far exceed their costs and may even exceed the advantages of competitors. In this way, winners may turn into losers in the capitalist economy. As Storper and Walker $(1989,48)$ put it: "capitalism is a mode of production in which 'all that is solid melts into air'. Simply making the best use of current resources - the neoclassical vision of economic rationality - is not the point; overcoming present limits to growth is." This dynamic disequilibrium model of competitiveness and economic growth manifests itself in space in the form of a process which Storper and Walker call leapfrogging. With this they mean the process by which (certain) lower-order centres, regions or nations, take over the lead of established ones every time new major innovations break through and new industrial ensembles emerge (Storper and Walker 1989, 11-18).

It is these dynamic disequilibrium forces, growing out of created absolute advantages, to which this section refers for a theoretical explanation of how businesses in non-central locations gain a competitive edge over firms located in wellprovided-for agglomerated environments by counteracting territorial resource scarcities. To that end, it must be shown that overcoming the limitations of the regional production environment generates important differential advantages. This, in turn, can reverse an initially bad position to create a leading position'. As Porter (1990b, 78) states: "a [factor] disadvantage in a static model of competition can become an advantage in a dynamic one." $\mathrm{A}$ number of examples of this reversal will be presented below. 


\section{Advantages induced by labour market constraints}

In the early 1980s, Quekel and Kragtwijk of System House Comdes had the problem that they could not find sufficient highly educated automation staff within the regional labour market (i.e. in the Province of Brabant). Furthermore, there was no opportunity to contract out automation training of people who lack knowledge in the field of computer science. In those days no such facilities existed in the local region. In response to the regonal employment situation Quekel and Kragtwijk had no choice but to make people acquainted with the field of computer science themselves. They decided to invest in developing an automation course for new personnel. Subsequently, the two men hired unemployed but highly educated people and gave them computer training in-house. When other organizations got wind of Comdes's automation training activities, they approached the software company to provide computer courses for their staff as well. Kragtwijk and Quekel complied with these requests. Training activities eventually developed into one of Comdes's core activities and main money makers (Vaessen and Wever 1990, 82).

The discovery of new business opportunities is but one advantage of tackling labour market constraints by means of extra internal education efforts. Several studies have examined labour market behaviour and training efforts of small innovative and high-technology companies in agglomeration areas on the one hand and outlying rural areas on the other. These studies reveal that the latter rely to a greater extent on internal labour markets and have higher training efforts than the former. This is precisely because of constrained external employment conditions (cf Oakey and Cooper 1989, Angel 1991, Meyer-Krahmer 1985). And it is precisely these greater training efforts and reliance on internal labour resources, commanded by external constraints, which possibly may give rise to extra differential advantages. For, it is widely recognized by economists and administrative scientists that investing in human capital by providing company training programmes and creating internal labour markets has an advantage over tapping external ones. The former alternative encourages motivation, initiative and creativity of workers to a greater extent than the latter (cf Lazonic 1989, 87; Reich 1989, 1990; De Smidt 1991). This effect is also clearly demonstrated by director Jansen of Casolith Sheets (Vaessen and Wever 1990, 47).

A third advantage which may rise out of shortages of suitable personnel is that this constraint can lead to technological innovation. For example, severe labour shortages lead many firms in Japan to invest heavily in labour saving equipment, particularly industrial robots. This is likely to further strengthen an economy that is already murderously competitive in export markets around the world (Newsweek 27-1990, cf also Porter 1990a).

\section{Advantages induced by financial environment constraints}

The sample firms also provide some examples of environmental resistance strategies from which differential advantages may be derived. Consider, for instance, the fact that director Jansen decided to let the employees take over Casolith Sheets when he couldn't find sufficient external investors who were prepared to buy shares (see Section 5.3). This step, born of necessity, may well have engendered differential 
advantages since businesses with employee ownership tend to perform better than businesses where this is not the case (Voute 1991). This is mainly because employee equity participation enhances motivation and commitment, and thus raises productivity.

Another example in the sphere of finance is provided by director Weishaupt of Rood Testhouse. The firm has had great difficulty in obtaining sufficient investment capital in the infancy stage. Weishaupt: "For financing we have crossed the whole country". After four years of searching and not giving up, an important breakthrough occurred (see Section 5.3). When asked whether these problems had seriously damaged the growth of the firm, Weishaupt answered: "Looking back, I would say that this has not occurred. At the time, it was very distressing.You do not renlize that you are undergoing an important leaming process. You get to know so many people who turn out to be very adoantageous afterwards".

\section{Advantages induced by market constraints}

As indicated above, one possible adaptation to local resource constraints consists of extending the operational space and tapping extra-regional sources. This was done particularly with respect to limited sales opportunities in the regional business environment. The Randstad businesses in the sample still clung to the surrounding regional home market very strongly at least ten years after their foundation. Yet from the outset non-Randstad firms operated mainly on extra-regional if not foreign markets. This finding corroborates that of Meyer-Krahmer (1985), who notes that innovative firms in rural areas with adverse structures have a higher outward orientedness than innovative firms in dense agglomerations. Peters $(1990,203)$ observes that the faster-growth firms serve a wider spread of markets. Furthermore, "it is clear that this wide spread of customers served .... resulted in the growth of the high fliers [i.e. rapid-growth firms] rather than vice versa" (ibid). Thus, Peters suggests that firms derive growth incentives from surmounting space and distance. In a similar vein, Kotabe and Czinkota (1992) find that export significantly benefits a firm's competitiveness. Export improves sales, managerial skill and a firm's competitive position.

In contrast, spatial researchers perceive the distance factor as merely a source of extra production costs, and as the main factor inhibiling remote firm growth. In the following, attention will be given to the advantages which can be reaped from overcoming the distance barrier. So, the extra expense incurred by tapping extraregional resources instead of regional ones should not be viewed as costs but as investments. To an extent, it is surprising that this viewpoint has not made its way into in regional science. Spatial scientists have recognized expenditures to overcome other kinds of barriers and time lags as investments from which differential advantages may be reaped. A notable example forms the mastering of technological barriers by means of R\&D investments.

Future business development attached to operating in an extensive market space has some advantages over concentration on the same output in one single but affluent region. One potential advantage is that this may improve a firm's reputation and thus strengthen its position within the home market. This effect has been observed with respect to firms engaged in export activities (Daems and Douma 1989, 
Bakker 1980, Porter 1990a), but it may well apply to an interregional market outlook as well

Secondly, having a foothold in several regions of industrial activity may well be more conducive to a firm's knowledge of the technical and customer standards which govern the market than concentrating all the sales in one area All of the most advanced and sophisticated buyers are rarely located at home, even under the best of circumstances (Porter 1990a, 607-608) The most demanding customers are not exclusively located in a country's economic heartland For example, regions with stnct environmental policies may be marked agricultural or rural areas, where there may or may not be severe environmental pollution Similarly, product requirements reflecting specific structural, cultural and especially natural conditions which prod innovation, need not run parallel to the centre-periphery dichotomy within a nation Obviously, what applies to a firm with sales spread nationally applies to an even greater degree to a firm having its sales dispersed internationally

Thirdly, and perhaps more importantly, highly outward-oriented firms will encounter a diversity of market needs due to legal, cultural, sectoral, natural and other differences between nations and regions This diversity will be greater than that confronting their more inward-oriented counterparts So, the former group of firms may develop greater product and process flexibulity in order to deal with these differences Director Aalberts of Aalberts Industries provides a clear example of this In the beginning of the 1970s, during a business trip in the United States, Aalberts came across a small firm which produces moulds by means of aluminium extrusion, which are sold to the aviation industry Aalberts was convinced that he could do the same, and he set out to study how to process aluminium In the beginning, the business did not come off the ground very smoothly, and hefty losses were incurred Aalberts himself travelled throughout the country, trying to sell his profiles In this way he gained a great deal of information about the variety of wishes and requirements of different types of potential customers Concerning this early start-up period, Aalberts (FEM, 25-2-1989, 68) sald "We learned a lot Because of the expertence we gamed then, we now have become one of the few compantes in Europe, which in close consuliation with the client are able to produce talor-made products of alummum and steel " Director Aalberts has made good use of these expensive lessons in the early beginning, since his company has grown very successfully

Fourth, that which applies to the most demanding and elaborated standards as well as for diversity of market needs also applies to new developments Firms with an extensive action radius are more likely to be directly confronted with new developments at an earlier point in time than businesses with a more limited action radius (cf also Porter 1990a, 59) This may favour the tmming of their innovations An example is provided by director Kragtwijk of the Comdes Group A major specialization of System House Comdes is engineering software for hospital automation, which the firm markets under the label Rodias The initial software was developed in response to an order by the Lukas Hospital in Amsterdam, which in the early 1980s started automating its radiology department Subsequently, Comdes was successful in selling Rodias on the German and Belgian markets as well "Thereby," Kragtwılk said, "we try to introduce the experience ganted in Germany, Belgum, and even in Oman here it the Netherlands You only have to look, for example, at a stmple thing like cancer registration In 
Germany this has been required by law for five to six years already. Over there, they know exactly how to do this. In the Netherlands, no initiatives have been taken on this yet, but the time is drawing near. By now, we are already introducing the German system here in Holland. Well, in that way you gain a certain advantage over [domestic] competitors".

Another example of this is provided by Nedap. In the late 1960 s director Paijens came into contact with chips technology as well as with synthetics technology for the substitution of metal and Bakelite casings by synthetic ones. This took place via very good relations the company maintains with Swiss watchmakers such as Omega. Via a subsidiary of Omega, Paijens got hold of an early electronic watch, made in Japan, and a synthetic one, made in Switzerland. From the Swiss, Paijens learned how to assemble synthetic coverings. However, to grasp the technology of the electronic part of the new Japanese watches Paijens requested professor Rodenburg of the University of Twente to give him more information. Rodenburg took Paijens and some of his fellow workers along with him to U.S. and showed him Silicon Valley, particularly Stanford Research Institute. This occurred in 1972. Paijens was very impressed. Paijens: "They showed us the articles which were going to capture the fut ure. We got the fright of our lives over there." The long and the short of this is that Nedap was able to apply chips technology in the products it manufactured in the early 1970s (e.g. switches). The company saw opportunities to apply integrated circuit technology in the field of electronic detection systems. However, in those days, the market was not ripe for this yet. Paijens: "We took our ideas out on the rond but couldn't sell anything." Nevertheless, the breakthrough of the company as a successful micro-electronics firm was only a matter of time. In 1976 its time had come, though rather unexpectedly. Paijens: "During a birthday party a fellow worker heard somebody telling that there was a need for a system with which cows could be automatically fed with concentrates in a programmed way. Our man responded that Nedap possessed the right knowledge for developing such a system. The day after, that person showed up on our doorstep." The result is that the company started developing a so-called responder, an electronic system for identification in the form of a collar. Paijens: "It proved that our system lent itself perfectly to this. A year thereafter we had drawn in orders worth three million guilders. We were the first in the Netherlands with a good system for administering feed concentrates automatically" (cf also NRC 18-11-1987; Paijens 1987). By 1987 Nedap had about 40 per cent of the world market for electronic collars.

A final example which demonstrates that a high outward-orientedness is a prerequisite for determining the right time to launch new products relates to Bronkhorst High Tech. BHT's major competitors are located in America. According to director Jouwsma, his firm has a lead of two years over the Americans. In order to retain this advantage, Bronkhorst must make sure that it has finished developing a new generation of instruments on the right time. Jouwsma: "Thus, the moment they show signs they are ready to come onto the market with a new product, we are able to deliver ours instantly."

Timing is intertwined with a fifth important source of advantage for firms with a wide geographical (market) outreach relative to their counterparts, who are focused on their home region. The former enjoy so-called first-mover advantages associated with earlier extension of the action space. First-mover advantages are benefits which accrue to a firm not because it is better but because it is earlier than a competitor in taking a 
particular action (Porter 1985). This type of advantage arises when firms move first or early into new technologies. However, according to Porter (ibid), the same advantages can also accrue to the first firms to move into a geographical area. First-mover advantages are particularly important in that they are not very susceptible to rapid imitation behaviour by competitors. The next section will devote more attention to this issue, i.e. the (un)susceptibility of catch-up strategies to imitation by contenders.

\subsection{The sustainability of revenues from adaptation behaviour}

Let us recall the hypothetical two-firm case, consisting of one central and one remote firm. The latter does not resign itself to its locational disadvantage and takes certain innovative steps which make it strongly competitive. ${ }^{3}$. The former, perceiving this, does not just stand around watching. It can restore its initial comparative advantage due to its location by simply imitating the strategic step of the disadvantaged firm. In this way, it can achieve the same revenues. For example, imagine that it proves that employee ownership, introduced by a peripheral firm because of lack of alternative sources of finance (e.g. Casolith Sheets, page 120), enhances its performance. Once aware of this, the central firm may also adopt this form of finance. Thereby, it reestablishes its initial comparative advantage.

Another example might be the case of a promising lean-area firm initiating robotization in response to labour shortages in the external environment. Its competitor in the centre sees this occurring and fears being outstripped by this manoeuvre. It decides to follow its remote contender and starts robotizing too. Carrying through the innovation is likely to be more efficient in the central-area firm than in the one far away, for two reasons. First, the outlying-area firm is the first to develop robotization of the production process and therefore incurs pioneering costs (see Porter 1985). The central-area firm does not, since it may imitate this innovation (so-called second or late-mover advantages). Secondly, because of a wide range of factor advantages, it is much easier for the central-area firm to call in external support to overcome barriers and difficulties encountered in carrying out this innovation than it would be for its peripheral counterpart. Thus, the innovation process is likely to take place more smoothly and efficiently in the central firm than in the outlying one. As a result, the catch-up attempt does not get the latter firm anywhere. On the other hand, we should realize that this is only a theoretical case. In practise, firms may not always react so quickly to each other, for of all kinds of rigidities may interfere. For example, the central-area firm may become aware of the catch-up strategy of its disadvantaged contender rather late. It may underestimate the strategic move of its competitor. Or it may be unwilling to give up some of its initial comparative advantage, and so forth and so on. As a consequence, the innovating peripheral-area firm in our example may enjoy a competitive edge over its centrally located competitor for a considerable period of time. Nevertheless, this lead remains vulnerable to imitation by competitors.

This is, however, not the whole story. In some instances, imitation behaviour on the part of the central-area firm may not suffice to close the gap created by a disadvantaged firm which has taken the lead by making a quantum leap. This is the case when first-mover advantages arise. 
Porter (1985) mentions several types of potential first-mover advantages which may accrue to a firm taking a particular action first. In this section, only some of them will be mentioned. Here the accent lies on firms moving first into a geographical area.

\section{Pre-empting markets and resources}

A first (or early) mover may pre-empt resources, an attractive product, or market position, and thus force followers to use or adopt less desirable ones. This applies particularly to highly specialized niche markets, where resources are scarce on a world-wide basis. An example is Bronkhorst High Tech. The entire line of business in which the firm operates comprises only 13 firms, of which BHT belongs to the topfive in terms of annual sales volume. And according to Jouwsma, the market cannot accommodate more businesses. After ten years of entrepreneurship, Jouwsma and Bruggeman are convinced that they started their firm at the right moment. "Now, it would not be possible any more. The pie has been divided up. No room is left for a newcomer" (van Berkel 1991, 17). For the same reason, it is fortunate that Bruggeman and Jouwsma did not delay in addressing foreign markets. As from the very start-up, BHT still operates mainly on an international market. It now sells its products virtually world-wide.

\section{Channel selection and market expansion strategy}

The firm first operating on a extra-regional market can pick the best brokers, distributors, or retailers to deal with distant customers. Or it may pilfer the most attractive opportunities to address distant markets from right under the nose of its competitors. A case in point, is again Broukhorst High Tech. In the beginning, the company used the more limited but more accessible international laboratory market as a launch pad for reaching the more prosperous but less accessible OEM market. Had Bruggeman and Jouwsma started their firm at a later point in time, the laboratory market would probably have already been exhausted by a predecessor. It would no longer be a useful springboard to reach the OEM market. Consequently, Jouwsma and Bruggeman would have been confronted with the difficult task of dealing with the OEM market directly.

\section{Institutional barriers}

Porter (1985) notes that being first in a country may give a company special status with the government. The case of Intercai suggests aiming at such an advantage, though not only with respect to entering a foreign country; for example, the annual report of 1989 showed a picture of the Belgian minister M. Colla visiting Intercai Belgium. It also seeks that advantage when it enters new domestic regions. On 18 December 1990, for instance, Intercai Utrecht moved to new premises. The new office was opened by the Mayor of Utrecht, Mrs. M.W.M Vos-Van Gortel.

\section{Spatial learning advantages}

Small firms selling in distant markets have another important though intangible advantage over their counterparts whose trade is concentrated (mainly) in one region. 
The former have already taken a difficult hurdle which the latter still have to surmount if growth is to be sustained. In other words, the former have a learning advantage in regard to addressing distant customers, who are difficult to reach? There is a large element of risk attached to such efforts. This is especially true when it comes to developing export markets. The expenditure can be considerable for small firms, while the sales potential is uncertain. However, even centrally located firms eventually have to break out of the local business region if they intend to continue growth. Promising peripheral firms are forced to take this step much earlier in their life cycle, if not from the beginning. This is because of limited market opportunities in their home region. However, if they succeed in accomplishing this, they may reap important competitive advantages simply by taking this step earlier than their central counterparts. From Taylor (1975) we may deduce that such spatial leaming ${ }^{5}$ constitutes a learning process by which firms expand their operational space and thereby cross progressively larger thresholds, i.e. local, regional and national boundaries. So, a firm may have to go through a process of geographical expansion within the domestic market before it is prepared to cross the national boundary. Welch and WiedersheimPaul (1981) and Wiedersheim-Paul et al (1983) refer to this process as domestic internationalizntion, reasoning that the domestic expansion process can be characterized as a type of internationalization. They argue that as a firm expands its operations into more distant regions, it moves into less familiar territory, i.e. more 'foreign' markets. Communication is more difficult and costly than for the local region. But as these barriers are overcome, the relative 'foreignness' of distant markets is reduced ${ }^{6}$. Also, the firm is likely to develop skills in marketing a product at a distance. The first mover begins down the spatial learning curve earlier than his followers. For the latter, it is difficult to reach the same level, since spatial learning is a matter of learning-bydoing or learning-by-experience (cf Denis \& Depelteau 1985, Welch and WiedersheimPaul 1981). And that is a highly individual or localized process. Because of these features of learning-by-doing processes, spatial learning advantages are sustainable and difficult to overcome by competitors (cf Stiglitz 1987). The occurrence of spatial learning processes is clearly suggested by an Australian investigation. This research reveals that non-exporters take almost three times as long as exporters to expand interstate on the domestic market (Welch and Wiedersheim-Paul 1981).

\subsection{Summary}

Although locationally disadvantaged firms may have higher production costs than businesses in well-provided-for areas, these higher costs do not necessarily prohibit the former from being able to outcompete the latter. The success of the sample firms is based on their superior market position. That superiority is the result of absolute or differential advantages, which eclipse the role of production costs. This explains the viewpoint of director Aalberts of Aalberts Industries. When asked whether the fact that many business services cannot be found in the business region is harmful to business performance, Aalberts replied: "It is all the same to us whether such businesses are located in the vicinity of the firm or elsewhere. We simply let them come over here rather than having to travel to Amsterdam ourselves $[181 \mathrm{~km}]$... That really causes no problem." 
Aalberts gives the impression that he considers the extra call-out charges as a joke. Against the background of high incomes such costs are 'peanuts' For the same reason, other directors in the sample can brush aside supposed disadvantages of having matenal suppliers that are not located in their vicinity (cf Chapter Five, Section 5.4) Actually no sample firm operates at maximal cost efficiency Yet this does not prevent them from growing bigger and bigger, thanks to their high incomes resulting from their superior market position Thus, for example, Bronkhorst High Tech's growth is not curbed by the persistent, wasteful difficulties the company had in implementing the nght business automation system System House Comdes, in its heyday, could permit some bungling to find the right organizational structure without hampering company growth (Vaessen and Wever 1990) The labour market deficiencies which Waterland Automation encountered in the mid-1980s may have slowed down company growth, according to Schoneveld, but certainly did not block it And although Infotheek cannot hedge fully against delivery uncertainties caused by its hardware supplier (IBM), the company has nevertheless been growing very rapidly

The one-sided concern with the costs of the 'distance' factor, leads to a significant narrowing of the scope of regional development theory, especially in regard to flexible agglomeration theory Regional scientists only consider the extra costs outlying area firms have to incur to deal with greater distances relative to their more favourably located counterparts With that focus, they fall to give consideration to the extra revenues which may be gained in overcoming this hindrance This chapter has given special attention to promising peripheral area firms which, dnven by local resource scarcities, are forced to extend their market outreach beyond the boundanes of their regional home base earlier and more intensively than their favourably located counterparts We have argued that those firms can thus reap important differential or absolute benefits Such advantages are highly insensitive to the higher production costs which may be attached to a place of location outside a country's economic heartland Moreover, because these benefits include learning advantages, they may be immune to imitation strategies and therefore remain sustainable This is what Porter (1990a, 64-65) makes contending that the differential advantages of moving early in international competition can generate leadership positions which can be held for decades, if not longer

One point remains to be addressed, however It cannot be denied that the responsiveness of small firms towards environmental limitations merely reflects the strength of the companies' human and material resources The crux of the matter is, of course, that these firms' capabilities do not appear out of thin air The last question to be posed in this research therefore concerns the ultimate origins of the resilience observed among the surveyed firms

\section{Notes}

1 The role of pressures and factor disadvantages 15, however, not unconditioned, according to Porter Factor disadvantages must be selective to motivate and not discourage, involving some but not all factors A lack of pressure means that there is rarely progress, but too much adversity leads to paralysis An intermediate level of pressure, involving a balance of advantage in some areas and disadvantage in selected 
others, seems to be the best combination for improvement and innovation. Furthermore, disadvantages in basic and generalized factors (such as the absence of domestic raw materials or shortages of low and semiskilled labour) and high absolute factor costs can be much better circumvented by means of innovation relative to disadvantages with respect to advanced and specific factors, such as highly and narrowly skilled workers, technological infrastructure and scientific knowledge bases in particular fields. Therefore, while the former adversaries act as triggers, the latter function as inhibitors of innovation and advancement. Thus, "A nation's firms succeed in an industry because pressures are juxtaposed with some advantages in responding to them, such as sophisticated local demand, a highly developed supplier base, and specialized factor pools (Porter 1990a, 174)." So, even though Porter keeps repeating the message that competitive advantage results from overcoming barriers instead of using advantages, ultimately his perspective is ecological, or - using Porter's own terminology - Ricardian (ibid, 173).

2. It may be clear that this ability to turn negatives into positives is not granted to every firm. Otherwise small-business growth in peripheral areas would be a phenomenon much more common than it actually is. After all, in itself the occurrence of environmental barriers does not trigger differential advantages; businesses must also liave the right capacities and sufficient means to master these constraints.

3. This is not to suggest that only scarcity of extemal resources stimulate innovativeness and that munificence does not. Arguing this would be ridiculous as in general the majority of innovative businesses are located in well-provided-for metropolitan heartlands. This applies even to the Netherlands (Davelaar 1989). In regional science several theories explain this phenomenon (Davelaar 1989, Malecki 1991). What is contended in this text is that innovations and advantages may arise both from harsh' and from 'benign' conditions in the local business environment. For example, whereas a lean-area firm having its buyers widely dispersed across space may have better knowledge of the diversity of customer demands, a central-area firm having its clients located close by may have more intimate knowledge of customer demand. Differential advantages may be reaped from both factors.

4. On the other hand, central area firms are more likely to grow larger in their business region, i.e. to accumulate more assets, than their peripheral contenders. Thus, the former may be better equipped to undertake the venture of breaking out of the home region. However, if first-mover advantages in entering a geographical space do exist, the central-area firms may need these extra resources badly as well, since late movers may face distinctive disadvantages which lead to higher costs (Porter 1985). This is aggravated by the fact that the market capacity outside of the core region may be so limited that in some countries central-area firms virtually have to jump from the regional to the international level when expanding their market space. It obviously requires large amounts of moneyand skills to accomplish this. On the other hand, if these central-area firms prove to be able to realize such a giant step across space, they may well have compensated for falling behind the outward-oriented peripheral-area firms in the spatial learning process.

5. The term spatial leaming has been borrowed from Harrison et al. (1979). 
6. Apart from communication (and information) barriers, the so-called threshold fear forms another important barrier in the spatial learning curve. The threshold fear relates to the attitude and orientation of the decision-maker with respect to addressing distant markets. Thereby, Wiedersheim-Paul et al. (1983) see not only export markets as 'foreign' or distant, but also regions outside of the local environment. They define interiational orientation as: "the extent to which a decision maker perceives and considers as interesting events occurring outside his own country - or perhaps even outside his own local environment" (ibid, 48). Overcoming threshold fear is the first priority of non-exporting firms with the potential to actually become exporters (Dichtl et al. 1983). It has been shown that unless decision-makers possess certain opinions and attitudes, firms will not respond adequately to export opportunities, such as an unsolicited order from a forcign customer, nor will they adequately exploit internal potentials, such as the unique characteristics of their product (Dichtl et al. 1983, 1984). Furthermore, it appears that exporters have a higher level of education, less fear of the risks involved, lower cost estimates of export activities, and less fear of communication difficulties than their colleagues concentrating on the local market. 


\section{Chapter Seven}

\section{Environmental responsiveness of small firms (III): its origins}

\subsection{Introduction}

In attempting to discover the origins of the high level of adaptability and fitness of the sample firms, our attention is drawn to their history. After all, the ability of businesses to respond effectively to comparative disadvantages is dependent on their accumulated stock of assets, both financial and non-financial (e.g. experience, skills, knowledge). So a company's present adaptability results from a company's past success (see Levinthal 1991). Consequently, to trace the ultimate origins of a (contemporary) business's resilience, we have to review the determinants of its initial growth. The money made in this incipient period governs the organization's scope for subsequent development and survival. It determines the range of options available for organizational reflection and problem solving; it sets the stage on which management can act. In sum, the slack resources created in the infant growth phase define the opportunities for future survival and growth of a new firm. Growth, in turn, is dependent upon the firm's success in marketing the goods and services produced. The impact of the initial market position on the chance of survival can be illustrated in the light of an example borrowed from Levinthal (1991, 399). The first spreadsheet program for the MS-DOS operating system, developed by Lotus Corporation, was a phenomenal success. This successful initial product introduction buffered the company from imminent failure despite the turbulence of the computer software industry and Lotus's subsequent difficulty in introducing new products and extending its product line.

The only viable way to generate enough money to cover the cash demands of the pioneering phase is by obtaining enough orders. Easy access to customers is crucial to rapid growth. The adage that business growth starts with access to markets is meant not in a deterministic but in a facilitative way; that is, market access is a necessary but not sufficient condition for sustained growth. All the sample firms, irrespective of whether or not they ran into trouble afterwards, got past the phase of early growth.

But what are the factors out of which initial success in the marketplace evolves? For the sample firms two sources influenced the market position of start-up firms, both lying outside of the companies' walls. One is the regional environment or ecology. 
Obviously, small firms located in a metropolitan core region, where large numbers of potential buyers are concentrated, may enjoy a major advantage over firms in the periphery. Those in the core may receive a greater impetus for growth because they are in much closer proximity to the market. Remember that a wide gap was observed between the opportunity for centrally and peripherally situated firms to rely on local customers. Proximity can facilitate access to buyers. Along the lines of spatial monopoly hypothesis in regional science (McDermott 1974, Greenhut 1974) Porter (1990a, 1990b), argues that this is because distance to potential buyers affects the producer's knowledge of their demands and needs. He suggests that the closer the potential buyers, the more intimate the producer's market knowledge will be, the better the manufacturer is able to adopt, and the more ready is his access to the market. Waterland Automation seems to lend support to this line of reasoning. The automation firm was founded in response to its founder's knowledge of the difficulty that locally based large manufacturing enterprises experienced in detaching from central computer centres for administration automation in the 1970s.

As head of the 'process automation' department of the fairly big metalworking company VMF Stork in Amsterdam, Pieter Schoneveld had to deal with business automation. Schoneveld: "In those days [mid-1970s] mini-computer systems arose, which basically enabled organizations to break away from central computer centres. We conducted relatively much research into this phenomenon. Thereby we could also observe that this decentralization of business automation conused conflicts between the computer centre and the organization. This induced me to start a business of my own, to further develop administrative business automation.... To me it was perfectly clear that the preference to undertake automation at firm sites, which the rise of mini-computer systems made possible, was an enormously rapidly growing market segment."Schoneveld appeals to the personal connections established in this period of research to get the first customers. His idea proved to be sound. Schoneveld: "I had no difficulty in finding customers, who were located in Amsterdam - where I started the company - and in the middle of the country, in Utrecht [37 km] and its surrounding area."

Not only was the perception of market opportunities underlying the start-up of Waterland Automation grounded within the business region. Also the initial trigger for acquiring a unique software product, called IMPCON, which gives Waterland a differential advantage, comes from the immediate business environment. After all, requests by customers - who were all locally based in the beginning - to implement production control systems on mini-computers led Schoneveld in 1981 to start investigating American Material Resource Planning (MRP) system software, albeit without much success. However, via his contacts with English software companies also dating from his period at Stork VMF - Schoneveld got into touch with a London company, Computer Systems Development (CSD). It appeared that CSD was ahead in developing MRP software. CSD was one of the first companies that was able to bring an MRP software package onto the market, called IMPCON. Waterland purchased IMPCON and was able to adapt it to the Dutch market successfully. IMPCON became the main money maker of the company. 
The case of Groenendijk plc demonstrates how the density of the regional sales market can trigger business growth, in that market opportunities virtually impose themselves upon producers. Groenendijk was established in 1947 in Rotterdam by Mr. M. Groenendijk as a one-man transport business. This situation remained the same for about 20 years. Then, in the second half of the 1960s, the firm gradually expanded to about six or seven trucks, and director Groenendijk took on some drivers. An important breakthrough did not occur until the early 1970s. In those days Groenendijk transported mobile huts for construction sites, which are used by house painters, building contractors, and the like. Director Groenendijk Jr. "From the contacts with these people, at a certain point the idea emerged to let out these mobile make-shift accomodations as well. This meant the start of the leasing company. Hiring out mobile site huts was something very new in those days. The market developed as we and our colleagues began to offer these huts to let." Furthermore, Groenendijk emphasizes that the combination of the transport activities and the rental enterprise is very fortunate. This makes it possible both to serve customers immediately and to provide day and night service. Anyway, the new enterprise was quite successful. The growth of the company then received a second, even larger impulse. It was discovered that the mobile huts can be made suitable for other uses besides construction works. Through standardizing the size and shape of the units, coupling them and piling them up, almost any type of accommodation can be created in any size desired. So the mobile site hut for construction works developed into a cabin which can also serve as a temporary shop, school, and many other uses, even as a mobile operating room. The density of the local market again played an important role in this process of successful product development, as can be inferred from Groenendijk's account. "The one who was the first to hire a temporary shop from us was not approached by us. We did not know that we were leasing temporary shops. So not we, but the guy who rented from us was the one with the idea to use our mobile site hut as a temporary shop. But we energetically picked up this idea. This is the way it has occurred in many instnnces." Both valuable ideas, i.e. renting mobile huts to construction firms and adapting these huts to suit other potential users, emerged directly out of local market linkages, within the Greater Rotterdam area. It is much less likely that this feedback would also have occurred if the firm had been located outside of the Randstad, all else being equal, since the density of economic activities, and thus potential customers, is significantly lower in those areas. What is more, Groenendijk ple grew into the largest firm in its kind of business in the Netherlands merely on the basis of its sales to the local region. It has already established national market leadership before the owners decided to invest in penetrating domestic regions outside of the Randstad and the Belgian market.

In the case of Database Consultancy Intemational in Amsterdam, the density of the local economy has worked in a less direct manner, namely via employee relationships, to get the firm off the ground. Director De Zoete: "We lave always been focussed on large customer firms. In the beginning. Albert Heijn and Shell were very important clients. At that time, we were able to pull in Shell [The Hague, $57 \mathrm{~km}$ ], because a number of workers who joined our company already maintained good relations with Shell. This has been very important in establishing a good foundation for further development." Clearly, the greater the density of the regional economy, the more likely such opportunities will occur. By the way, DCI succeeded in acquiring Albert Heijn 
(Zaanstad, $15 \mathrm{~km}$ ) as an early customer via the relationship between the founder of DCI, i.e. Mr. Greystoke, and De Zoete, who was an employee of Albert Heijn in those days.

Furthermore, Randstad companies appear to rely heavily on 'personal selling' to gain clients. Take Waterland Automation again as an example. Schoneveld: "Personally I am convinced that up to now personal selling has been the most important aspect of our company." Director Van Leenen of the Infotheek Group seems to subscribe to this view. As outlined above in Chapter Five, van Leenen thinks that he probably would not have achieved the same growth with Infotheek if he had been located outside of the Randstad. Van Leenen: "Outside the Randstad, distance would turn up as a limiting factor. Because our customers are located mainly in the Randstad, a location elsewhere in the country would have become problematical. For face-to-face contacts with clients play an important role in our line of business, and traffic jams would pose great difficulties in visiting them." Definitely, dense clustering of potential customers facilitates going after orders by means of personal selling.

The regional ecology plays an important role in getting access to growthinducing clients of only one non-Randstad firm. This company is Intercai. Intercai's headquarter is now located in Geleen, but in the beginning it was not. The company started out in a small South Limburg village called Beek, a stone's throw from Maastricht $(10 \mathrm{~km})$. Over one year after its administrative foundation and about half a year after it was made operational, business was running smoothly for Intercai. Two major orders laid the foundation for this. The first was an assignment by the city of Heerlen $(15 \mathrm{~km})$ to assist with the construction of a local cable television network. According to Kivits'account: "We knew that the city of Heerlen had plans for the construction of a cable television network. In the Province of Limburg, few cable television mains had been constructed yet. As a consequence, little expertise existed in those days. Our nearest competitors were located in Arnhem $[139 \mathrm{~km}]$ and Gouda $[201 \mathrm{~km}]$ or somewhere over there. The local conncil of Heerlen had to weigh the pros and cons between calling in broad experience from far away or placing their trust in guys nearby with little experience but who showed enthusinsm and personal commitment.... I think that we managed to convince our client that the risk of taking their chances with us was lower than the risks posed by the long distance from other consultants. Apparently this did work." Kivits succeeded in gaining a second important order on New Year's Eve in 1981. The city of Maastricht intended to construct a cable television network as well. Kivits: "In Maastricht, I could, of course make a long story short. To them I could say: look, in Heerlen we are alrendy doing such-andsuch." These two big orders laid the basis for drawing in other projects as well, including a very large order in 1983 by the provincial government of Limburg in Maastricht. This meant the company's 'decisive' break through.

Thus, it has been shown that proximity and thereby the local ecological setting can play a decisive role in triggering initial firm growth. This seems to lend support to Porter's stance that businesses are only able to overcome certain environmental disadvantages if they can take advantage of other elements of the environment in which they are located (see Chapter Six note 1). This is surely true of the national economic environment, to which Porter primarily refers. However, it does not suffice to explain (non-basic) business growth in peripheral regions within countries. This is because regional development theorists assume that many peripheral areas have 
disadvantages over (nearly) the entire range of factors of production, This includes at least the most important ones, such as skilled labour, information and know-how, services, advanced material supplies, and customers. So there must be some other force which sets the ball rolling for successful outlying-area firms. A number of sample firms suggest that to identify this force, one has to go back to the incubator or parent organizations from which these enterprises emanated.

\subsection{The incubator organization}

For the four Randstad firms as well as Intercai, the regional setting has been an important factor in creating initial market access and early firm growth. In contrast, five (younger) non-Randstad firms in the sample, namely Aalberts Industries, Bronkhorst High Tech, Comdes System House, Elephant Industries, and Rood Testhouse, were from the start able to make do without the benefits of many local sales opportunities and still achieve a rapid growth. They had to, since sales opportunities are virtually absent in their place of business. From the outset, these latter businesses succeeded in penetrating national and even foreign markets. At first sight, they seem to have ignored spatial learning processes and relegated the concept of the spatial learning curve to the realm of academic fantasy. Actually, however, these companies' founders had already gone down this curve a long way before they started their own firm. This occurred during their previous employment, which was in nationally or internationally operating companies. The following examples elaborate on their backgrounds.

Before he started his own enterprise, Aalberts (Aalberts Industries) was vicepresident of the very internationally oriented, medium-sized, high-tech metalworking company Indivers plc (for more information about Indivers ltd the reader is referred to Van Twaalfhoven 1984). Bijsterbosch and Weishaupt, directors of Rood Testhouse, got to know each other at Philips Data Systems in Apeldoorn. There both men gained experience in testing electronic systems. They wanted to specialize further in this kind of activity and they wanted to switch over to a small (less than 100 employees) intermational trading company of electronic products, including testing systems. This company is C.N. Rood in Rijswijk (near The Hague), which forms part of the medium sized high-tech-group Phoenix Megatronica plc, to which Hulo International belongs as well. Quekel and Kragtwijk of System House Comdes had made a tour of five or six early and renowned Dutch automation companies, such as Volmac and Raet, prior to self-employment. In this period, Quekel mainly had commercial functions, while Kragtwijk had technical ones. Bruggeman and Jouwsma (Bronkhorst High Tech) came from a manufacturer of measuring and regulating equipment, named Brooks Instruments. Brooks is a Dutch subsidiary of the American Rosemount Measurement and Control Instrumentation Group. Bruggeman fulfilled the function of delegated director and Jouwsma was head of the R\&D department. Director Brouwers of Elephant Industries, finally, was head of the commercial department at the Dutch subsidiary of a French precious metalworks.

This in itself suggests that these entrepreneurs possessed many relations and contacts on a supraregional and even international level through their prior 
employer(s), even before they decided to start their own business (for further evidence of this see Chapter 5, Section 5.6). Thus, it is the incubator organization where the entrepreneurs have developed their outward-orientation and have surmounted the threshold fear in addressing foreign and distant markets. It is also through their previous employer that they discovered the niche or opportunity which they currently exploit. There they also gained a thorough knowledge of the standards which have to be dealt with in the national and international environment. And the organization the entrepreneurs came from is where they established the concrete business relationships to actually enter into distant markets. Take, for example, Bruggeman and Jouwsma (Bronkhorst High Tech) who knew the first sales reps for penetrating the Dutch and German markets from their previous posts at Brooks Instruments, and, perhaps more importantly, the brokers knew them. In the process of learning by doing at the parent organization, the entrepreneurs have overcome communication barriers with foreign customers and other business relations. Thereby, according to Kaynak and Kothari (1984), communication problems surpass verbal language barriers and impose other problems of a more subtle but pervading nature, such as symbolism in body language, non verbal-cues and responses, methods of doing business, and practices involving business relations and protocol. In sum, their outward orientation and their domestic and international contact networks allowed the entrepreneurs ofthese five businesses to surpass the limitations of their local business region, even at the beginning. Actually, none of these enterprises could ever have got off the ground if the operational space or the communication networks of the firms' founders had been confined to the regional scale.

As has been argued above, it is clear that the entrepreneurs developed their wide geographical outlook and linkage network in their incubator organizations. They then incorporated this feature into their own enterprise when they started it. In this way, the new firms inherited their extensive task environment, and thus their accessibility across space, from the organizations they grew out of. We may call the incubator organization the second source of initial market access and, consequently, of incipient small-firm success.

Peters $(1990,196)$, who studied small-business growth, also contends that the incubator organization is a source of success. He states that "the nature of the incubator firm will influence the new founder's choice of product and his or her knowledge of market opportunities. Such opportunities need not necessarily be local: whilst most small firms tend to serve local markets (...), it is usual for innovative ones to supply non-local customers (...). Indeed, knowledge of such non-local markets for innovative products may well be gained from employment in local firms already serving distant markets." This may explain why the most rapidly growing firms, the so-called high fliers, tend to spin out from larger incubator organizations, particularly public and foreign-owned companies (ibid, 322). Cooper (1985) and Cooper and Bruno (1977) obtain similar results. These insights fit in with $O^{\prime}$ Farrell's and Hitchen's finding of a foreign dimension in the competitiveness of small (Irish) firms ( $O^{\prime}$ Farrell and Hitchens 1987). They observe that, generally speaking, the products of small Irish firms are less quality-competitive relative to small Scottish firms. However, those Irish companies that are competitive reveal a remarkable overseas influence. This occurs either directly, when the company in Ireland is owned and run by a foreign propri- 
etor who has been trained abroad, or indirectly, through training and work experience abroad or being trained in an MNE branch in Ireland. "In this way they were made aware of international standards of training, productivity, quality, design and delivery" (ibid, 412).

In this context, Peters (1990) as well as Cooper (1985) stress the powerful influence that incubator organizations may exert on the nature and growth perspectives of their spin-offs (see also Cooper and Bruno 1977). In sum, there may be some transmission of attributes from parent organizations to their offspring. Actually, this phenomenon is more familiar in economic and regional science than one would think at first sight. Anyhow, one feature is widely recognized to be transferred from parent organizations to their spin-offs. This is the choice of the new firm's product (Peters 1990). Wever (1986), for example, finds a very strong relationship between the industrial composition of new firms and the existing firms in a region, with Pearson correlation coefficients of at least 0.90 .

A second group of attributes carried over from the parent to a descendant organization is organizational form and structure. Brittain and Freeman (1980), examining the development of the semiconductor industry, note, for instance, that semiconductor firms founded by persons from larger and older companies who come. out of manufacturing tend to have a production efficiency style of operation and structure. In contrast, those founded by persons from engineering and research and development are organized somewhat more organically and have a product strategy emphasizing technical innovation.

The present study adds to this a third attribute that may be inherited by new firms, namely their geographical outlook or, in other words, the nature and spatial extent of their linkage network'. In sum, there may be instances of what Betton and Dess (1985) term a gentotype, spreading from incubator organization to its spin-offs in a way that success literally may breed success.

Anyhow, the point is that new entrepreneurs do not start out as a tabula rasa. Instead, prior to start-up they may already possess certain valuable assets of a tangible (monetary) and especially an intangible nature. The starter can capitalise on these assets and thus withstand initial pressures from the production environment, including limited local market opportunities. In Figure 3.6, these assets have been termed pre-existence and start-up capital. They are made up of material (or tangible) and organizational (or intangible) resources.

Recapitulating the findings on the external sources of early firm fitness, the possibility arises to supplement the ecological model (Lambooy 1984) with a heredity model. Drawing together these two kinds of forces creates a naturalistic framework ${ }^{2}$ for the explanation of initial small-firm success. In contrast to the ecological perspective, this wider framework may also account for peripheral small-firm growth. From such a naturalistic model of the forces behind initial firm growth, it follows that the existence of new promising companies in outlying areas depends on the presence of appropriate incubator organizations. These carry over the right properties to their spin-offs and allow them to escape the stronger selection pressure from the environment in these regions. In more favourable ecologies, such as large cities and industrial districts, in contrast, where selection pressures are weaker, new entrepreneurs can manage on less capabilities ${ }^{3}$. In such environments relatively poorly equipped entre- 
preneurs may also get the chance to grow rapidly, though sometimes not until they have led an inconspicuous life for years, as is the case with Groenendijk plc. This is due to local opportunities and support which a firm in a central, metropolitan milieu is more likely to find in its path than would its counterpart in a leaner, outwardoriented environment".

There remains one subject yet to be dealt with in this research. This is the stormy weather a number of sample firms have landed in recently, including the complete break-down of three of them.

May I reiterate that the above explanation of central versus peripheral-area firm growth only explains the triggering of initial business growth. This early growth provides the basis for developing enterprise resilience and adaptability in a more advanced stage of firm development. From that point onward, a behavioural or adaptation approach may become increasingly adequate to explain firm performance. From this perspective, I can agree with Kimberly (1980), who finds that early success in the founding of an organization (i.e. an innovative medical school) is incompatible with its requirements for survival in the long run. The process of getting off the ground is strongly influenced by external forces, since the requisite assets, capabilities, or whatever may be needed to get production going, does not appear out of the blue. Instead, these inputs must be tapped from sources outside of the new firm. The potential of these external sources is largely evinced by initial business sales.

However, successful passage through the creation phase enhances only the potential subsequent staying power of an organization. In general, as firms grow, the accumulation of resources increases the scope for strategic adaptation and self-control. What actunlly occurs, whether or not a company is able to follow up its initial growth, however, depends on management decisions and organizational aspects. Consequently, as organizations mature, success and failure become more of an internal affair and less of an external one. For no president of Philips or IBM or any other established enterprise, can be excused when his organization gets into serious trouble and suffers heavy losses because of external head-winds encountered of whatever kind, wherever they come from. And there is absolutely no excuse for trouble due to local constraints. Research on firm failure has revealed that factors internal to the organization exert more influence than external factors in cases of bankruptcy (De Graaf and Jaspers 1989, GRAYDON 1991a, 1991b).

Those sample firms which have got into trouble confirm this picture. Notwithstanding, external factors, such as worsening market conditions, did play a role in the decline of these firms as well. In this respect it is telling that five out of the six companies which ran into a crisis after the start of the research in 1988 are in the computer industry. It is common knowledge that the latest economic recession hit the computer sector earlier and harder than many other sectors of business activity. However, on closer scrutiny market decline only explains a small part of the businesses' distress. This applies most clearly to System House Comdes, as this company was already in trouble in 1987, prior to the start of the decline in computer business. (For 
more detailed information about Comdes's decline see Vaessen and Wever 1990.) Also with respect to Infotheek it is clear that developments in the industry cannot explain the company's failure. Infotheek's downfall occurred too rapidly to blame the industry. Within a time interval of only six months, the company turned from the nation's market leader in its field of business into a bankrupt estate (Het Financieele Dagblad 5-11-1991). The major direct cause of Infotheek's collapse, which received abundant attention in the media, is the failure of the company's internationalization strategy (Het Financieele Dagblad 29-5-1991). With respect to the other two automation firms which went out of business, Waterland Automation and Database Consultancy International, as well as the hard times Rood Testhouse is currently in, external forces probably played a greater role in these companies' declines. These firms would most likely have stayed in business or would not have experienced such severe decline if market growth had continued. On the other hand, the worsened external conditions laid bare some serious internal flaws, such as poor anticipation by the management and/or taking restructuring measures too late. Here we will not explore these weaknesses ${ }^{6}$.

In sum, this section emphasizes that the naturalistic framework, addressed in the previous section, only serves to explain the perspectives for initial business growth, not for eventual growth. In other words, it is suggested that exogenous circumstances (environmental and/or progenitory), take precedence over endogenous forces (i.e. management and/or organization) to influence initial competitiveness and the market position of new small businesses. But this relation (gradually) reverses after the first substantial growth has been achieved. By then, in principle the assets have been accumulated to exert self-control and adapt to external pressures. The present research cannot and does not pretend to explain small business success and resilience in the long term. At most, it attempts to identify the major ingredients which lay the basis for initial growth capacity. So, as a consequence, irrespective of whether or not sample companies eventually got into trouble or even went bankrupt, they have experienced substantial growth. By that very fact, they belong to the select ensemble of promising small companies out of which an even more select group will achieve long-lasting growth, and out of which only one or two may go on to become giants.

\section{5 \\ Summary and conclusions}

The research question dealt with in this chapter considers the origins of the adaptability of the businesses investigated. It has been determined that adaptability starts with easy market access, allowing a firm to generate a sufficient cash flow to build an adequate financial and organizational basis. The (younger) sample companies' first successful market access can be traced back to two basic sources:

(1) the regional ecology, and in particular its endowment with potential buyers;

(2) the incubator organization, and in particular its spatial action radius and linkage networks.

This suggests that there is scope in regional science to supplement the prevailing ecological perspective in regional science with an economic heredity approach. Both 
approaches can be drawn together in a naturalistic framework of external sources of promising entrepreneurship. A purely ecological perspective strongly favours smallfirm growth in a nation's economic core areas. The heredity force - grafted onto the role of the incubator organization - offers an explanation for the phenomenon of noncentral business growth. Conversely, the lack of promising new firm activity in noncentral areas can be attributed to either inadequate environmental conditions or to inappropriate incubator firms. The latter are inappropriate in that they spawn too few new firms with the right attributes to overcome or even take advantage of suboptimal conditions in their environment. The question arises whether the finding that incubator organizations hold the seeds of small-business success is a fruitful clue for policy-making in peripheral areas. This question will be dealt with briefly in the closing chapter.

\section{Notes}

1. In regard to the survey firms, one observation seems to contradict the suggestion that new firms inherit their market space from their incubator organizations. This is that the founders of Randstad firms such as Waterland Automation, Database Consultancy International, and Infotheek maintained national and international contacts in their positions prior to self-employment. This may be inferred from the following statements. Schoneveld: "Sirce 1975, I maintain quite intensive relations with American software companies." Van Leenen, who comes from Control Data, said: "I have taken a long time for fact finding, to see what's what in this line of business. When I went on a business trip to America, I alwways took a week off as zuell, to look for opportimitics." The intemational scope of Mr. Greystoke, founder of DCI, is self-evident. Greystoke himself is an Englishman, managing the Dutch subsidiary of an American automation company. In spite of this international dimension in these entrepreneurs' backgrounds, their firms' sales linkages are strongly concentrated on the regional market, although some export does take place. A similar finding has been observed by Peters (1990). A high proportion of successlul businesses located in the economic heartland of Great Britain, i.e. the prosperous South, sell their products within their own county and region. In contrast, more peripheral North West firms sell outside their home region. This is surprising, considering that more owner-managers of Southern firms have an international background (i.e. they are more likely to come from foreign-owned plants). However, as Peters suggests, the higher market potential in the South East removes the incentive for Southern firms to look far outside their own region. On this basis it can be hypothesized that affluent conditions at home may induce firms to leave an initial potential to address far-away markets unused, whereas poor market potential at home just activates this potency. With that, just as in biological evolution theory, potencies lying idle cause their degeneration, whereas breaking into potencies may develop them further.

2. The terminology is borrowed from literary criticism. The naturalistic school dominated European literature from 1880 to 1900 . It considers man as a product of unrelenting social and natural forces. In the novels of Emile Zola, the founding father of literary naturalism, two conditions determine man: environment or milieu and heredity. 
3. Simultaneously, this may explain the lower failure rates observed in outward areas realitve to other regions (see Chapter One and also Westhead and Birley 1992).

4. On the other hand, a munificent environment may not only upgrade firms with moderate capacities, but it may also downgrade entrepreneurs or companies initially possessing great capacities, as has been argued in note 1 .

5. For further information about Infotheek's downfall, see Het Financieele Dagblad 31-101991, 5-11-1991; NRC Handelsblad 29-5-1991, 31-10-1991; De Volkskrant 15-6-1991, 296-1991; Trouw 5-11-1991.

6. For more information about the decline of Waterland Automation and Rood Testhouse the reader is referred to Vacssen and Wever 1990 (Ch. Seven) and newspaper reports (Het Financicele Dagblad 7-9-1991, 22-4-1992, 27/29-6-1992, 7-9-1992, 8/10-8-1992, 5-111992; De Volkskrant 27-6-1992; NRC Handelsblad 25-6-1991) respectively. 


\title{
Summary and conclusions
}

\author{
8.1 Summary
}

\section{Introduction}

Though prosperity is a quality generally attributed to distinct nations, its origins are not. Regional scientists observe that wealth is not produced nationwide but in one or a few subnational regions, designated as economic heartlands or core areas. They point out that the underlying processes of industrialization and economic growth are spatially concentrated. Consequently, theorizing in regional science has come to focus almost completely on processes of polarization and inequality across space. It is argued that territorial polarization and inequality are inevitable conditions of growth itself. And at the same time, it is acknowledged that economic advancement emerges from technological innovation. Thus it is argued that core regions offer environmental conditions which are important to organizations' innovativeness. Recently, this view has been applauded by experts in the area of management science.

However, clustering of innovative activities is not a hard and fast rule. In reality, many businesses are able to start up and grow outside of a country's economic heartland. In some countries, such as in the Netherlands, the concentration of promising businesses in a few select regions is hardly observed. In other instances, there are large and unspecified gaps in the explanation of the location of economic activities. But even if non-central areas prove to accommodate low percentages of growth-inducing activities, the absolute number of firms may still be considerable. Their presence poses a challenge to prevailing theoretical insights about the importance of territorial conditions for innovation and competitiveness. And that phenomenon forms the point of departure for this thesis: the formation and growth of small firms in contrasting regional environments, that is, in central as well as peripheral regions. The aim of the book is to find an approach to the clarification of business success in disparate environments. Accordingly, the general research question is: What is the underlying reason why some firms in non-central areas are no less competitive than their counterparts in the centre? This broad research question has been split into three more specific questions. These are:

1 How do prospective firms respond to economic conditions in the production environment, both opportunities and limitations? 
What can be said about the strength or the efficacy of environmental responsiveness of small firms?

3 What are the ultimate sources of the firms' environmental responsiveness?

\section{Firm and environment in regional science}

In order to meet the research objective, first an analysis is conducted on the views prevailing in regional science about the role of the environment relative to the organization in explaining business growth. Basically, spatial theories can be arranged into two broad lines of approach. One line assigns primacy to the environment over the firm (i.e. location theories and regional development theories). The other, in contrast, assigns primacy to the organization over the environment (behavioural and particularly corporate geography). Environmental or ecological approaches have come to dominate theorizing in the field of regional science. Initially, traditional location theories ran the show, but gradually regional development theories took over this position. These latter theories concentrate on developmental differences across space between prosperous central and backward peripheral regions. Regional development theories have in common the central role of agglomeration advantages for economic development. The concept of agglomeration economies supposes that spatial propinquity allows better access to the inputs and outputs necessary for production, thereby raising revenues, lowering costs and increasing productivity (Walker 1986, 248).

Behavioural and organizational approaches, on the other hand, have developed in response to criticisms of the shortcomings of ecological approaches. One such criticism concerns the subservience of the individual entrepreneur to the dictates of the surrounding economic environment. In location theory as well as regional development theory, the individual entrepreneur is deprived of any ability to counteract the constraints of the production environment. Organizational and behavioural approaches accuse ecological approaches of environmental determinism. Nevertheless, the former never succeeded in providing an adequate alternative for the latter in regional science, particularly not for regional development theories. This is because the geography of enterprise lacks an appropriate theoretical framework itself. Three shortcomings have been highlighted in particular. The first is the concentration of corporate geography on the big multinational company and its neglect of the small firm, which makes up the vast majority of profit-seeking organizations. A serious implication of this bias is that corporate geography fails to deal with the origins of growth. The second point of critique is that the geography of enterprise treats firms in isolation. As a consequence, corporate geography pays insufficient attention to processes of interfirm competition. Third, the geography of enterprise offers an inadequate definition of the environment a firm has to deal with from a spatial point of view. It tends to focus on geographical attributes of the environment as outcomes of organizational behaviour, passing over the role of unequal input conditions across space. As an input condition for organizational behaviour, the environment is conceived of in terms of non-geographical attributes such as environmental dynamism and uncertainty, if indeed it is taken into account at all. The effect of this omission is that corporate geography does not develop a stance concerning the impact of comparative and agglomeration advantages on business development, which is a 
crucial theme in the field of regional science.

In order to explain company growth outside of a country's favoured core areas, it must be demonstrated how small businesses can resist and adapt to locational disadvantages while competitors enjoy comparative advantages. For developing such an adaptive or organizational approach in regional science, urban field theory forms a useful basis and starting point. It allows the broad framework to be deduced, within which opportunities arise for greater business autonomy vis-à-vis environmental conditions. The urban field approach links the opportunities for non-central business growth to the degree of national economic and technological advancement. In the developed nations, economic facilities are so accessible and dispersed so widely that even deep in the periphery business growth is not inhibited by environmental conditions. This is not to say that unequal economic conditions across space have been evened out. Rather, it means that in the periphery, certain minimum requirements have been met which make it possible for certain outlying area-firms to take measures to overcome any locational disadvantages. In this way, urban field theory creates room for more managerial or organizational autonomy vis-à-vis the surrounding environment. In order to take advantage of this space and to buttress such an approach in the field of regional science, we have drawn some lessons from a different economic discipline which also deals with firm-environment relationships and interactions, namely organization theory.

\section{Organization and environment: a perspective from organization theory}

The quest for useful ways to incorporate greater organizational autonomy in regional science yielded the following conclusions. First, out of the constellation of environmental forces, those which originate in the business region, as a rule, will be less compelling than those which originate in the macro environment.

Second, munificence rather than dynamism or complexity is the crucial dimension of the environment which regional scientists have to deal with. After all, it is not so much the variability (dynamism) or the heterogeneity (complexity) but rather the available abundance (munificence) of the requisite resources that differ across space. Organizational approaches in regional science do not seem to recognize this.

Third, within the field of organization theory resource dependence theory emphasizes the reactive organization as an intermediate form between the proactive and the inactive organization. At first sight, it looks as if regional science only acknowledges the latter two characteristics imputed to organizations. Corporate geography is preoccupied with the proactive firm, whereas location theory and regional development theory are focussed on the inactive or powerless firm. However, on further consideration, by a stretch of the imagination, we can name one early offshoot within the geography of enterprise school which does deal with a kind of reactive organization. This offshoot, which has remained underdeveloped in regional science, could be called adaptation geography. The concept of the reactive organization assumes that firms are affected by the environment without being determined by it. In line with this, adaptation geography describes how organizations (can) respond or adapt to environmental forces, paying special attention to spatial adjustments. This somewhat premature line of thought in industrial geography contains two major 
flaws. One is that it treats the adaptive organization in isolation. The other shortcoming is its inadequate treatment of an organization's environment from a regional science point of view. The adaptation approach in corporate geography conceives of the environment as a source of uncertainty instead of scarcity. Consequently, adaptation geography bypasses the essential issues of comparative advantages and agglomeration economies.

Fourth, the numerous ways in which firms can respond to environmental forces have been grouped into four basic categories. These are: manipulation, immunization, adaptation, and utilization.

Fifth, while regional science is in the grip of the dichotomy between the big powerful corporate organization and the restrained small firm, thus obstructing the development of an adaptation approach for small firms, some organization theorists have paved the way for such a perspective. This is based on the recognition that although small firms possess less power to deal wilh external forces, they also make fewer demands on the environment. Therefore, they are exposed to fewer external pressures than big enterprises. So, although there irrefutably exists a wide gap in absolute power between large multiplant companies and small single-plant firms, the relative gap may be much smaller. If small companies' adaptive capacity exceeds a minimum level, they may be able to cope with the environment in accordance with their needs for survival. This is the basic notion which underlies the developmental model of organizational adaptability. Therein, a modest initial adaptive ability expands along with an organization's growth, the resulting rise in its external requirements, and the associated increasing environmental pressures.

\section{Research method and sample}

The empirical part of the research attempted to demonstrate the environmental responsiveness of the reactive small and medium-sized firm. To achieve this, it was decided to use a qualitative research method. Such a method involves the collection and analysis of qualitative data. Qualitative data are detailed, concrete, non-metric descriptions of people and events. So the research sought to obtain in-depth knowledge about the interactions between firm and environment by means of semistructured face-to-face interviews with the directors of small and medium-sized firms. As a consequence, only a limited number of cases were investigated, albeit quite extensively. The thesis took a qualitative case-study approach because of its great potential for accuracy and specificity in understanding causal mechanisms. On the other hand, its reliability and representativeness is more difficult to assess than in a quantitative method, which is able to express these matters statistically. Nevertheless, various measures were taken to gain insight into the reliability and the representativeness of the research findings. Concerning reliability, the research used triangulation of interview questions and member checks, and also supplied precise information about the methods and procedures used. Representativeness rests on the logic of the inferences made as well as on comparison of the results with the outcomes of other investigations. Despite these measures, only future research of a quantitative nature will be able to measure the reliability and representativeness of the research findings more adequately. 
The survey sample comprises 13 growth-oriented small and medium-sized firms. All but one of these are medium and high-technology companies. The sample includes enterprises in the fields of micro-electronics, metal components, synthetics, transport equipment, services related to computer software and hardware, telecommunication consultancy, and rental of temporary business accommodation. Four of the firms are located in the polycentric metropolis in the western part of the country known as Randstad Holland, which constitutes the national economic heartland. The other nine firms lie in the more rural northern, eastern, and southern peripheries of the country. With regard to age, the sample includes nine younger and four older enterprises. Of the younger companies, the founders were interviewed.

\section{Environmental responsiveness of small firms (I): its forms}

This chapter deals with research question number one, elucidating how small firms respond in practice to the conditions in the external environment, both opportunities and handicaps. In regard to the process by which the sample businesses satisfy their resource needs, three observations were made. First, in addition to Randstad firms, non-Randstad companies take considerable advantage of local resources. Centreperiphery contrasts seem to be moderate in the Netherlands. Secondly, this contention needs to be qualified. On the one hand, the equal satisfaction of production requirements across space is related to the growth stage of a firm. As firms mature and professionalize, they raise their demands on quantity as well as the quality of external inputs and deliveries. Ultimately, a greater resource capacity of central areas may come to light. On the other hand, interregional convergence of economic conditions differs with respect to type of resources. Centre-periphery contrasts are greater and more discriminating in regard to local sales opportunities than to input resources. The third observation is that these comparative disadvantages did not prevent the peripheral firms from being competitive and successful. In general, the sample firms revealed a considerable ability to overcome the constraints of the production environment. They did so by manipulating external resource conditions, by immunizing themselves against them, or by adapting to them. In practice, the ability to cope with centre-periphery contrasts in availability of factor resources manifests itself most clearly in non-Randstad businesses that adopt a wider geographical market outreach earlier in their development than Randstad firms. This has been interpreted as a form of immunization against limited local sales opportunities.

\section{Environmental responsiveness of small firms (II): its strength}

This chapter dealt with research question number two, concerning the effectiveness of entrepreneurial jostling vis-à-vis the environment, as this can drive production costs upward. It should be noted that not all acts to counter external constraints involve high expenditures. For example, a firm may benefit greatly from a good reputation as it seeks to satisfy its needs and fulfil its requirements. Furthermore, in a small, affluent country like the Netherlands, production costs presumably differ moderately between regions. But even if these costs are comparatively high relative to another 
region, due to locational disadvantages, nothing is proved until one considers the returns from adaptation strategies. There is evidence that businesses can gain absolute or differential advantages from overcoming comparative disadvantages. Differential advantages are benefits which are so great that they outweigh the drawback of higher production costs. It was argued that dealing with locational handicaps may yield important differential advantages. Special attention was devoted to the advantages that can be reaped from overcoming limited local sales capacity. It was reasoned that small companies which are forced to spread their sales widely across space and therefore gain a foothold in several geographical markets, as a rule, will possess higher market intelligence than their counterparts, which concentrate their output in their home region. A wide geographical market outreach may alter a company's insight in both domestic and international production and consumer standards, its flexibility in dealing with different customer requirements, as well as the timing of innovations and strategic actions. Furthermore, an outward-oriented company can reap important benefits from the very fact of having to expand its market reach earlier than locally oriented firms. These gains, which have been labelled first-mover advantages across space, are of particular importance in that they are resistant to catch-up attempts of contenders and therefore are quite sustainable. Anyhow, the central message of this chapter is that, under certain circumstances, environmental constraints in peripheral areas can provoke organizations to use catch-up strategies which improve their differential position, whereby the drawback of higher production costs attached to such regions is eventually outweighed.

\section{Environmental responsiveness of small firms (III): its origins}

Chapter Seven considers research question number three, which concerns the ultimate sources of the sample firms' environmental responsiveness. The ability to turn comparative disadvantages into differential advantages can not be taken for granted by any firm. Otherwise small-business growth in outlying areas would be much more common than it is now. It was determined that adaptability starts with easy market access. This allows a firm to generate the cash flow to build an adequate financial and organizational basis for further action. The earliest successful market access of the (younger) firms in the survey was traced to one of two basic sources. The first of these is the regional ecology, and in particular a region's endowment with potential buyers. For all the Randstad firms and one non-Randstad company in the sample, the initial success in customer acquisition resulted from contacts and processes within the home region.

The second basic source of first thriving market access is the organization the entrepreneurs came from. The impact of the incubator or parent organization was clearly revealed by five younger peripheral sample businesses. From the outset, these companies proved to be able to counteract limited local sales opportunities by addressing extra-regional and even foreign markets. This is due to the extensive geographical scope their founders held at the time they started a firm of their own. It is quite clear that the entrepreneurs developed their wide geographical outlook and linkage network in the organizations where they worked before starting their own business. These incubator or parent organizations all operated on a national or 
international scale. The new entrepreneurs then incorporated this outward-orientation in their own enterprise when they started it. In this way, the new firms inherited an extensive task environment, and hence their knowledge of and access to extraregional markets, from the organizations they had grown out of.

This suggests that regional science might be able to supplement the prevailing ecological perspective with an economic heredity approach. Both approaches could be drawn together in a naturalistic framework of external sources of promising entrepreneurship. A purely ecological perspective is a forceful tool in explaining small-firm growth in a nation's economic core areas. But the heredity approach grafted onto the role of the incubator organization - offers an explanation for the phenomenon of non-central business growth. A policy issue then arises: does this finding that incubator organizations act as originators of small-business success offer a useful clue for policy-making in lagging regions?

\subsection{Conclusions}

\section{Implications for policy}

In relation to regional policy, the main question is what can be learned from the counterfactual businesses in the sample. These companies have shown an ability to maintain their productivity and competitiveness in 'unfavourable' production environments. Regional policy has long been directed at improving input conditions and lowering production costs to firms in policy regions. However, as Malecki and Nijkamp (1987) and Miller and Coté (1985) point out, it is impossible to find a substitute in smaller regions for the synergy found in large urban core areas. Analysing the sample firms, an alternative route is suggested for attempting to increase the number of new firms, and particularly their potential for growth. This alternative rests on two pillars. One is the familiar observation that the conditions of the production environment in less-developed regions deserve careful attention from policy-makers. Of course, we have no illusions that public policy can close the gap vis-à-vis metropolitan core areas in this respect. However, this is not really necessary. What is important is that economic infrastructure in peripheral areas reaches an acceptable or threshold level over a wide range of factors which penetrate deeply into the economy as well. The presence of that level of facilities would ensure that no bottlenecks prevent promising, small companies from taking appropriate measures to offset environmental disadvantages. Such an approach is in accordance with the idea expressed by Thoss (1984) about endogenous development promotion. Thoss poses that a strategy of endogenous development must also embrace a strategy aimed at the elimination of obstacles. Meeting minimum requirements for several relevant factor conditions in a policy region is more important than developing one particular factor to its optimum. With respect to education infrastructure, for example, it is more important to provide good education on a primary and secondary level in several fields for the great majority than specialized academic education for the happy few (cf Reich 1989; 1991).

The second tenet of such a policy involves improving the regional incubation structure for stimulating high-quality entrepreneurship in policy regions. Basically, 
this can be achieved in two ways. One way is for local authorities to attract organizations which are most likely to function as incubators of new firms. Their contribution will be greatest as incubators of new firms which prove resistant, to a certain extent, to the less-favourable circumstances in outlying territories. The present research suggests that these are the more outward-oriented start-ups. In order to improve the regional incubation structure in this respect, it is of utmost importance to identify which incubator organizations should be attracted. Our understanding of this is still very incomplete. Nevertheless, a few qualities which may make an organization a good incubator will be suggested below. One such attribute emerges from the present research: that these organizations retain a national or international outlook. Such organizations imbue local workers, including the potential entrepreneurs among them, with the abilities to gain access to extra-regional resources, particularly markets. These are abilities they need to overcome the constraints of the local business environment when they start a firm of their own. Another property of fertile incubator companies is that they should not be too large, nor functionally organized. Firm size and functional organization systems are generally inversely related to the number of new firms spawned (Cooper 1985, Malecki 1991). So, acquisition of large production plants of big foreign companies, which make up the bulk of acquisition policy in peripheral areas, will not stimulate new-firm formation. On the contrary, such a policy is likely to stifle this process. Instead, research laboratories, sales offices, and headquarters of international companies, as well as independent nationally or internationally operating small and medium-sized companies, are more eligible candidates for acquisition, as they might improve the regional incubation structure. On the other hand, such establishments are few and far between and, moreover, difficult to lure to peripheral areas.

However, there might be a second option for improvement of the incubation structure. The present research suggests that in remote areas, new firms, and particularly the more promising ones among them, come from outward-oriented parent organizations. So, it can be inferred that a widening of the action radius, and especially of the market space, of existing small peripheral-area firms will improve their incubation function. This relates to the fact that peripheral-area firms in general are much more locally oriented than central-area ones, despite the limited market opportunities in the home region of the former. This applies even in a small country like the Netherlands. It has been abundantly demonstrated that businesses in the northern policy regions display a much greater degree of parochialism with respect to their sales and business contacts than central-area companies (Pellenbarg and Zalme 1985; Buijs and Pellenbarg 1989; Dwars 1989; Machielse et al. 1990). It is necessary to break down this situation. Its demise would not only benefit the growth and competitiveness of the firms in question but would also enhance their function as incubators of prospective new firms. The first step to be taken in that direction is to stimulate and assist existing peripheral-area firms to extend their information and market space within the country. Only when such domestic expansion has been achieved is it feasible to stimulate those firms to cross international boundaries. After all, the mere use of incentives which are tied to international expansion (export) may be totally inappropriate when the target firms are in an early stage of domestic expansion (Welch and Wiedersheim-Paul 1980). Many policy measures can be designed to realize extra-regional expansion of locally bound peripheral-area firms. 
However, this issue is beyond the scope of the present research.

Furthermore, before such a policy can be initiated, much more research is required on the process of spawning new businesses, particularly of promising ones. This process will be affected by many more factors than the one discussed above, i.e. the operational space of the incubator firms.

\section{Suggestions for further research}

As the present research took an explorative approach (see Chapter Four), the points made in this thesis require additional substantiation and testing. The foregoing analysis suggest several points for further research with regard to small-business growth in contrasting environments, using either a quantitative or a qualitative approach or both. But the question remains whether an analogous approach in another country with greater centre-periphery contrasts than found in the Netherlands would have yielded similar outcomes and conclusions. It should be noted that the more frequent occurrence of innovative small businesses in non-central areas in the Netherlands relative to other industrialized countries is only a difference of degree. It is not a fundamental difference, as rapid business growth in outlying areas can be found in any developed country. Anyway, to validate the research outcomes and to further develop adaptation theory in regional science, in-depth research on similar firms in different regions should be conducted in other countries as well.

Secondly, at least three hypotheses can be inferred from the foregoing analysis. These require a quantitative underpinning. First, it was observed that rapidly growing firms in outlying areas tend to come from national or internationally operating parent organizations. The hypothesis has to be verified that they do so more than slowgrowth firms in the same territory and more than successful firms in central areas. Furthermore, it is expected that promising peripheral-area firms, driven by greater local resource scarcities, expand their operational space faster than promising centralarea firms (and, of course, also faster than unsuccessful peripheral colleagues). Thus, prospective small firms in outlying areas should have a wider action radius than their centrally located counterparts of the same age. Finally, the outward orientation of the former is expected to generate greater competitiveness in terms of timing of innovations and product variation.

Thirdly, more explorative research is required on the distinguishing characteristics of fertile incubation organizations as well as on the spin-off process itself. What makes organizations either good or poor incubators of new firms is a severely underinvestigated issue. Research efforts should focus on the role of parameters such as sectoral impacts, organization structure, age, business growth, growth strategy, corporate action radius, business size, and how these variables interfere with a firm's environmental setting. Thereby, attention should be devoted not only to the quantity of new firms spawned but also to their quality, i.e. their potential for growth and survival. Such studies can link up with organizational innovation research, as many new firms can be considered externalized innovations. The relevance of this kind of research to the field of regional science is illustrated by Scott (1988a, 91). He points out the potential role which particular administrative structures of incubator organizations may play in generating regional economic growth. To explain why Silicon 
Valley developed into the major hub of semiconductor industry, whereas initially conditions in other nascent centres of integrated circuit production (Phoenix and Dallas) were equally advantageous if not better than in Santa Clara County, Scott makes an interesting suggestion. The fundamental question is why Fairchild Semiconductors (in Silicon Valley) produced so many more spin-offs than similar firms such as Motorola (in Phoenix) and Texas Instruments (in Dallas). Scott suggests that the particular corporate structures of Motorola and Texas Instruments discouraged horizontal and/or vertical disintegration (spin-off) and encouraged the internalization of new product and process innovations. In contrast, Shockley Laboratories and later Fairchild Semiconductors just externalized these innovations. Unfortunately, according to Scott, the available data do not allow a more careful sifting of these issues (cf Scott and Angel 1987).

Fourth, there is a need to chart the growth path and growth strategy of small firms once they get started. In particular, more detailed knowledge is required about the growth of small firms across space. How do spatial thresholds to development (i.e. local, regional and national boundaries) affect the growth and expansion of small firms? And which organizational adjustments and changes are required to cross these barriers? Spatial science should have a better understanding of how the environmental setting of a firm interferes with the geographical evolution of its market space, in regard to both successful and unsuccessful firms. A useful method to investigate all these questions seems to be longitudinal matched pair analysis between central and peripheral as well as successful and unsuccessful firms which are of similar age and operate within the same sector.

In general we need more insight into why scarcity and constraints in the local area are destructive to many firms and prevent them from growing, while such adverse conditions just channel the growth of other, seemingly comparable, businesses organizations. What makes the latter different from the former? There is clearly much that spatial scientists can learn from deviant cases - that is, strong firms in unfavourable environments - no matter how many or how few there are. This applies not only to the further development of spatial theory but also, and perhaps more importantly, to the formulation of policy to stimulate industrial and regional development. 


\section{Bibliography}

ABLER, R.F. (1991), Hardware, software and brainware. In: D. Brunn and T.R. Leinbach, Eds., Collapsing space and time: geographic aspects of communication and information. New York: Harper Collins Academic, pp. 31-48.

ALCHIAN, A.A. (1950), Uncertainty, evolution and economic theory. Journal of Political Economy 58, pp.211-221.

ALDRICH, H.E. (1979), Organizations and environments. Englewood Cliffs, NJ: Prentice-Hall.

ALDRICH, H.E. AND S. MINDLIN (1978), Uncertainty and dependence: two perspectives on environment. In: L. Karpik, Ed., Organization and environment. Beverly Hills, Calif: Sage, pp. 149-170.

ALGEMEEN DAGBLAD (25-2-1989), Topprodukt in aluminium.

AMENDOLA, G., P. GUERRIERI AND P.C. PADOAN (1991), International patterns of technological accumulation and trade. Paper presented at the 18th Annual Conference of The European Association for Research in Industrial Economics. Ferrara, Italy, September 1-3.

ANGEL, D.P. (1989), The labor market for engineers in the U.S. semiconductor industry. Economic Geography 65, pp. 99-112.

ANGEL, D.P. (1991), High technology agglomeration and the labor market: the case of Silicon Valley. Environment and Planning A 23, pp. 1501-1516.

ANSOFF, H.l. (1968, 1987 revised edition), Corporate Strategy. London: Penguin Books.

APPOLD, J. (1991), The location processes of industrial laboratories. The Annals of Regional Science 25, pp. 131-144.

ASHCROFT, B., J.H. LOVE AND E. MALLOY (1991), New firm formation in the British counties with special reference to Scotland. Regional Studies 25, pp. 395409.

ASTLEY, W.A. (1985), The two ecologies: population and community perspectives on organizational evolution. Administrative Science Quarterly 30, pp. 224-241.

ASTLEY, W.A. AND VAN DE VEN (1983), Central perspectives and debates in organization theory. Administrative Science Quarterly 28, pp. 245-273.

AYDALOT, Ph. AND D. KEEBLE (1988), Technological trajectories and regional innovation in Europe. In: Ph. Aydalot and D. Keeble, Eds., High technology industry and innovative environments: the European experience. London: Routledge, Pp. 22-47.

AYDALOT, Ph. AND D. KEEBLE (1988), High technology industry and innovative environments in Europe: an overview. In: Ph. Aydalot AND D. Keeble, Eds., High technology industry and innovative environments: the European experience. London: Routledge, Pp. 1-21.

BAKKER, B.A. (1980), Export en marketing: enige aspecten van exportmarketing en exportsamenwerking bij Nederlandse ondernemingen. Alphen aan den Rijn: Samson.

BARTON, A.M. AND P.F. LAZARSFELD (1961), Some functions of qualitative analysis in social research. In: S.M. Lipset and N.J. Smelser, Eds., Sociology; The Progress of a Decade, pp. 94-122. Englewood Cliffs: Prentice Hall.

BEARD, D.W. AND G.G. DESS (1988), Modelling organizational species 
interdependence in an ecological community: an input-ouput approach. Academy of Management Review 13, pp. 362-373.

BERKEL, M. VAN (1991), De bewezen haalbaarheid van een onmogelijk ondernemersplan. Bronkhorst High Techin tien jaar naar branche-top. Elan, juli/augustus pp. 15-16.

BENSON, J.K. (1975), The interorganizational network as a political economy. Adminstrative Science Quarterly 20, pp. 229-249.

BERRY, B. (1972), Hierarchical diffusion: the basis of development filtering and spread in a system of growth centers. In: N. Hansen, Ed., Growth Centers in Regional Economic Development. New York: Free Press, pp. 108-138.

BETTON, J. AND G.G. DESS (1985), The application of population ecology models to the study of organizations. Academy of Management Review 10, pp. 750-757.

BUIJS, A.M. AND P.H. PELLENBARG (1989), Regional economic development and information technology. A case study of the Northern Netherlands. In: M. de Smidt and E. Wever, Eds., Regional and local economic policies and technology. Amsterdam/Utrecht/Nijmegen: Netherlands Geographical Studies 99, pp. 93-103.

BLEUMINK, P.H.M., G.B. DE GROOT, J. BILDERBEEK AND E. WEVER (1985), Nieuwe ondernemingen en regio. Een onderzoek naar de regionale verschillen in het startproces en de bedrijfsvoering van in 1975 of in 1980 opgerichte nieuwe zelfstandige bedrijven in de industrie, groothandel en zakelijke dienstverlening. Nijmegen: KUN.

BLUESTONE, B AND B. HARRISON (1982), The deindustrialization of America. New York: Basic Books.

BOSCH, F.A.J. VAN DEN (1989), Over de grenzen van organisaties, bedrijfskunde: organsatie, strategie en omgeving. Delft: Eburon.

BOUDEVILLE, J. (1976), French regional polarization and planning. Paris: Pion.

BOUDEVILLE, J. (1977), Functional regional analysis: an elementary exposition on some selected topics. In: J.H. Paelinck and A. Sailez, Eds., University of Toronto lectures. Paris: Association de Science Régionale de Langue Française.

BRAVERMAN, H. (1974), Labor and monopoly capital: the degradation of work in the twentieth century. New york: Montly Review.

BRITTON, J.N.H. (1974), Environmental adaptation of industrial plants: service linkages, locational environment and organizations. In: F.E.I. Hamilton, Ed., Spatial perspectives on industrial organization and decision making. Chisester: Wiley, pp. 363-390.

BRITTON, J.N.H. (1976), The influence of corporate organization and ownership on the linkages of industrial plants: a Canadian enquiry. Economic Geography 52, pp. 311-324.

BROWN, R.H. (1978), Bureaucracy as praxis: toward a political phenomenology of formal organizations. Administrative Science Quarterly 23, pp. 365-382.

BRUSCO, S. (1986), Small firms and industrial districts: the experience of Italy. In: D. Keeble and E. Wever, Eds., New firms and regional development in Europe. London: Croom Helm, pp. 184-202.

BUCK, R. (1988), Marktpositie en marktattractiviteit van Nederland voor buitenlandse investeerders. In: R. Buck and M. van Nieuwkerk, Red., Nieuwe buitenlandse bedrijven in Nederland: werving in het perspectief van 1992. Nijmegen/Den Haag, Vlaardingen: Buck Consultants International/American Chambers of 
Commerce in the Netherlands/Nederlands Studiecentrum, pp 11-30.

BUSINESS WEEK, (7 October 1990). Thınk Small, midsıze companıes give Germany's export powerhouse its punch, pp. $24 \mathrm{~B} / \mathrm{E}-24 \mathrm{~J} / \mathrm{E}$.

BRITTON, J AND J. GILMOUR (1978), The weakest link: a technological perspective on Canadian industrial development. Ottawa. Science Council of Canada.

CAMPBELL, D T (1965), Vanation and selective retention in socio-cultural evolution. In. H.R Barrınger, G.I. Blanksten, and R Mack, Eds., Social change in developing areas. Cambridge, Mass. Schenkman, pp. 19-49.

CARR, M. (1983), A contribution to the review and critique of behavioural industrial location theory. Progress in Human Geography, pp. 386-401.

CASTELLS, M., Ed. (1985 ed.), High technology, space and society. Vol 28, Berkeley: Sage.

CASTROGIOVANNI, G.J. (1991), Environmental munficence a theoretical assessment. Academy of Managment Review 16, pp. 542-565.

CBS (1991), Statistiek financien van ondernemingen Voorburg/Heerlen. Centraal Bureau voor de Statistrek.

CBS (1992), Volwasseneneducatie bedrijfsopleidingen 1990 deel 1, pariculiere sector. 's-Gravenhage: SDU.

CHAKRAVARTHY, BS. (1982), Adaptation. a promising metaphor for strategic management. Academy of Management Review 7, pp 35-44.

CHAPMAN, K. AND D.F. WALKER (1991), Industrial location principles and policies. Oxford Basil Blackwell.

CHILD, J. (1972), Organizational structure and strategies of control. the role of strategic choice. Sociology 6, pp. 2-22.

CHURCHILL, NC AND VL. LEWISS (1983), The five stages of small business growth. Harvard Business Review 61, pp. 30-50.

CLARKE, I M (1985), The spatial organization of multınational corporations. London. Croom Helm

COOPER, A C (1985), The role of incubator organizations in the founding of growthoniented firms Journal of Business Venturing 1, pp. 75-86.

COOPER, A. C. AND A.V. Bruno (1977), Success among high-technology firms. Business Horizons 20, pp. 16-22

COOPER, A C., W.C. DUNKELBERG AND R.S. FURUTA (1985), Incubator organizations. background and founding characterıstıcs. In. J A. Hornaday, E.B. Shils J.A. Timmons, Eds., Frontiers of Entrepreneurship Research. Wellesley, M.A. Babson College, Center for entrepreneurial sudies, pp 61-79.

CROSS, M. (1981), New firm formation and regional development Westmead Gower.

CRUM, R. AND G. GUDGIN (1977) Non-production activities in UK Manufacturing industry Brussels European Economic Community

CZAMANSKI, S. (1976), Study of spatial industral complexes. Halıfax, Nova Scotıa Inst. of Public Affarrs, Dalhousie University.

DANIELS, P.W. (1987), Supply and demand for intermediate services. an explanatory study of firms in Merseyside. Working Papers on Producer Services 7. Liverpool: Department of Geography, University of Liverpool.

DENIS, J.-E. AND D. DEPELTEAU (1985), Market knowledge, diversificaion and export expansion Journal of International Business Studies 16, pp. 78-86.

DICKEN, P. (1986), Global shift industrial change in a turbulent world. London. 


\section{Harper and Row.}

DICKEN, P. (1990), The geography of enterprise: elements of a research agenda. In:

M. De Smidt and E. Wever, Eds., The corporate firm in a changing world economy. London: Routledge, pp. 234-244.

DICHTL, E., M. LEIBOLD, H.-G. KÖGLMAYR AND S. MÜLLER (1983), The foreign orientation of management as a central construct construct in export-centred decision-making processes. Research for Maketing 10, pp. 7-14.

DICHTL, E., M. LEIBOLD, H.-G. KÖGLMAYR AND S. MULLLER (1984), The exportdecision of small andmediumsized firms: a review. Management Inernational Review 24, Pp. 49-60.

DILL, W.R. (1958), Environment as an influence on managerial autonomy. Administrative Science Quarterly, vol. 2, pp. 409-443.

DILL, W.R. (1962), Environment as an influence on managerial autonomy. In: S. Mailick and E.H. Van Ness. Englewood Cliffs, N.J.: Prentice-Hall, pp. 29-48.

DONCKELS, R. (1990), Gestart, maar dan? Rotterdam: NION.

DORSMAN, A.B. AND A. HUIZING (1989), Organisaties in het web van hun omgeving. In: A.B. Dorsman and A. Huizing, Eds., Organisaties in het web van hun omgeving. Lelystad: Vermande, pp. 1-5.

DOSI, G., K. PAVITT AND L. SOETE (1990), the economics of technical change and internaional trade. New York: University Press.

DOUMA, S AND H. SCHREUDER (1992), Economic approaches to organizations. London: Prentice Hall.

DUNCAN, R.B. (1972), Characteristics of organizational environments and perceived environmental uncertainty. Administrative Science Quarterly 17, pp. 313-327.

DWARS, R.F. (1989), Contactpatronen van bedrijven. Een verkenningvan de ruimtelijke spreiding van bedrijfsexterne informatiebronnen. Girug-serie 46. Groningen:RUG.

THE ECONOMIST (1990), Manufacturing: about time. August 11, p. 66.

EYLES, J. (1988), Interpreting the geographical world: qualitative approaches in geographical research. In: J.E. Eyles and D.M. Smith, Eds., Qualitative methods in human geography. Oxford: Basil Blackwell, pp. 1-16.

FEM (1989), Aalberts Industries zoekt uitbreiding in Europa. FEM (25 februari), pp. 68-69.

FEM (1992), Toeleverwereld is te klein voor Aalberts. FEM (13 juni), pp. 18-19.

HET FINANCIEELE DAGBAD (22-4-1991), Rood Testhouse boekt klein verlies in '91.

HET FINANCIEELE DAGBLAD (29-5-1991), Infotheek springt verder dan polsstok lang is: pc-distribiteur ziet buitenlandse expansie mislukken.

HET FINANCIEELE DAGBLAD (4-9-1991), Aalberts versterkt met VSH greep op markt.

HET FINANCIEELE DAGBLAD (7-9-1991), Rood Testhouse boekt negatief resultaat.

HET FINANCIEELE DAGBLAD (31-10-1991), Infotheek: 'slachtoffer financieel conflict'.

HET FINANCIEELE DAGBLAD (5-11-1991), 'Faillisement Infotheek onnodig'.

HET FINANCIEELE DAGBLAD (27/29-6-1992), Rood Testhouse vervangt voltallige directie.

HET FINANCIEELE DAGBLAD (8/10-8-1992), Rood Technology verplaatst produktie. HET FINANCIEELE DAGBLAD (7-9-1992), Rood Testhouse zoekt 
kapitaalverschaffers.

HET FINANCIEELE DAGBLAD (5-11-1992), Doek valt voor high-tech bedruf Rood Technology.

FREEMAN, J.H. (1982), Organizational life cycles and natural selection processes. In B.M. Staw and L.L. Cummings, Eds., Research in organizational behaviour. Geenwhich, CT. JAI Press, vol. 4, pp. 1-32.

FRIEDMANN, J. (1966), Regional Development Policy: A Case Study of Venezuela. Cambridge, Mass.. MIT Press

FRIEDMANN, J. AND J. MILLER (1965), The urban field. Journal of the American Institute of Planners 21, pp 312-320.

FROEBEL, F., J HEINRICHS AND O. KREYE (1977), The new international division of labor. Cambridge- Cambridge University Press (Engl. edition 1980).

FUCHS, M. AND E. SCHAMP (1990), Standard Elektrik Lorenz introducing CAD into a telecommunications fırm: its impact on labour. In M. De Smidt and $E$. Wever, Eds., The corporate firm in a changing world economy. London: Routledge, pp. 55-76.

GAEBE, W. (1988), Disparities in development between agglomeration areas in the Federal Republic of Germany. Zeitschrift fur Wirtschaftsgeografie 32, pp. 179-191.

GERLACH, L.P. AND G.D. PALMER (1981), Adaptation through evolving interdependence. In P.C Nystrom and W H. Starbuck, Eds., Handbook of organuzational design. Volume 1, Adapting organizations to their environments. London Oxford University Press, pp. 323-381.

GERLACH, M.L. (1992), The Japanese corporate network- a blockmodel analysis. Administrative Science Quarterly 37, pp. 105-139.

GERTLER, M.S. (1984), Regional capital theory. Progress in Human Geography 8, pp. 50-81.

GERTLER, MS (1988), The limits to flexibility comments on the post-fordist vision of production and its geography. Transactions Institute of Britısh Geographers N.S., Pp. 419-432.

GIBBS, D (1991), Venture capital and regional development the operation of venture capital industry in Manchester. Tijdschrift voor Economische en Sociale Geografie 82, pp 242-253

GILMOUR, JM. (1974), External economies of scale, interındustrial lınkages and decision makıng in manufacturıng. In F E.I. Hamilton, Ed., Spatial perspectives on industrial organization and decision making Chisester Wiley, pp. 335-362.

GLASMEIER, A. AND N. SUGIURA (1991), Japan's manufacturing system small business, subcontracting and regional complex formation. International Journal of Urban and Regional Research 15, pp. 395-414.

GODDARD, J.B. (1978), Office development and urban and regional development in Bntain. In: P.W. Danıels, Ed., Spatial patterns of office growth and location. London. Wiley, Pp. 29-60.

GORDIJN, H. AND L. VAN WISSEN (1992), Demografie van bedrijven een andere methode voor analyse en prognose van regionale economische ontwikkelingen. Planning 43, pp. 31-43.

GRAAF, LE DE AND P.F.M. JASPERS (1989),Falliet door mismanagement. NIBonderzoek naar de belangrıjkste faalfactoren.

GRA YDON, (1991a), Bijna helft van fallissementen gevolg van mismanagement. Het 
Nederlands Fallisementenregister 5, no 41, pp. 1-2.

GRAYDON, (1991b), Veel fallissementen kunnen worden voorkomen. Het

Nederlands Faillissementenregister 5, no 42, pp 1-2.

GRUIJTHUIJSEN, E. VAN AND P. JUNGE, (1992), De revolutie van Jan Timmer. Naarden. Strengholt.

GUDGIN, G. (1978), Industrial location processes and regional development growth. Farnborough Saxon House.

HAKANSON, L. (1979), Towards a theory of location and corporate growth. In F.E.I. Hamilton and G.J R. Linge, Eds., Spatıal analysis, industry and the industrial environment Vol. 1, Industral systems, London' Wiley pp. 115-138.

HANNAN, M.T. AND J.H. FREEMAN (1977), The population ecology of organizations. Amencan Journal of Sociology 82, pp. 929-964.

HANNAN, M.T. AND J.H. FREEMAN (1989), Organizational ecology. Cambridge, Mass.. Harvard University Press.

HANSEN, N.M. (1970), Development pole theory in a regional context. In. D L. McKee, R.D. Dean, AND W H Leakey, Eds., Regional economics. Theory and practice. New York: The Free Press, pp. 93-135.

HARRISON, R.T, P.J. BULL AND M HART (1979), Space and time in industrial linkage studies. Area 11, pp 333-338.

HARRISON, R.T. AND M. HART, Eds. (1992), Spatial policy in a divided nation. London Jessica Kingsley Publishers.

HAYTER, R. AND HD WATTS (1983), The geography of enterprise a re-appraisal. Progress in Human Geography 7, pp. 157-181.

HENCKEL, D (1989), Die raumliche Verteilung von Unternehmen der Biotechnik und der Informationstechnık. Ein Vergleich. Informatıonen zur Raumentwicklung $H$. 4, pp. 237-244.

HIGGINS, B. (1988), regional development and efficiency of the national economy. In. B. Higgins and D.J. Savoie, Eds, Regional development essays in honour of Françoıs Perroux Boston Unwın Hyman, pp. 193-224

HITOMI, K (1989), Non-mass, multi-product, small-sized production the state of the art. Technovation 9, pp.357-369.

HIRSCHMAN, A. (1958), The strategy of economic development New Haven Yale University Press.

HORVERS, A. AND E. WEVER (1987), De alerte onderneming. Economisch Statistische Berichten 26 August, pp. 795-798.

HORVERS, A. AND E. WEVER (1989), The alert firm spatial patterns and theory In: M. de Smidt and E. Wever, Eds., Regional and Local Economic Policies and Technology. Amsterdam Netherlands Geographical Studies, Pp. 131-148.

HREBINIAK, L.G. AND W.F. JOYCE (1985), Organızational adaptation. strategic choice and environmental determinism Administrative Science Quarterly 30, pp. 336-349.

HYMER, S. (1972), The multınational corporation and the law of uneven development In J. Bhagwal, Ed, Economics and world order. New York Free Press, pp. 113140.

HYMER, S. (1976), The international operations of national firms a study of direct foreign investments. Cambridge MIT Press.

ILLERIS, S. (1986), New firm formation in Denmark the importance of the cultural 
background. In: D. Keeble and E. Wever, Eds., New firms and regional development in Europe. London: Croom Helm, pp. 141-151.

INTERMAGAZINE (1986), De 1986 Intermagazine top-25. De vijfentwintig snelste kleine grote groeiers van Nederland. Intermagazine, November, p.39.

INTERMAGAZINE (1987), De 1987 Intermagazine top-25. De vijfentwintig snelste kleine grote groeiers van Nederland. Intermagazine, November, p. 39.

INTERMAGAZINE, (1988), De 1988 Intermagazine top-25. De vijfentwintig snelste kleine grote groeiers van Nederland. Intermagazine, November, p. 39.

JANSEN, A.C.M. (1970), The value of the growth pole theory for economic geography. Tijdschrift voor Economische en Sociale Geografie 61, pp. 67-76.

JONG, M.W. DE (1987), New economic activities and regional dynamics. Amsterdam: KNAG, Nederlandse Geografische Studies.

JURKOVICH, R. (1975), A core typology of organizational environments. Administrative Science Quarterly 19, pp. 380-394.

KASARDA, J.D. (1988), People and jobs on the move: America's new spatial dynamics. In: G. Sternlieb and J.W. Hughes, Eds., America's new market geography: nation, region and metropolis. New Brunswick, N.J.: Center for Urban Policy Research, pp. 217-242.

KAYNAK, E. AND V. KOTHARI (1984), Export behaviour of small and medium sized manufacturers: some policy guidelines for international marketers. Management International Review 24, pp. 61-69.

KEEBLE, D. (1986), The changing spatial structure of economic activity and metropolitan decline in the United Kingdom. In: H.J. Ewers, J.B. Goddard and H. Matzerath, Eds., The future of the metropolis: Berlin, London, Paris, New York: economic aspects. Berlin: Walter de Gruyter, pp. 177-199.

KEEBLE, D. (1988), High technology industry and local environments in the United Kingdom. In: Ph. Aydalot and D. Keeble, Eds., High technology industry and innovative environments: the European experience. London: Routledge, pp. 65-98.

KEEBLE, D. (1989), The dynamics of European industrial counterurbanization in the 1980s: corporate restructuring or indigenous growth? The Geographical Journal 155, pp. 70-74.

KEEBLE, D. (1993), Regional influences and policy in new technology-based firm creation and growth. Cambridge: Cambridge University Small Business Research Centre, Working Paper, 34 (forthcoming).

KELLY, T. AND D. KEEBLE (1990), IBM: the corporate chameleon. In: M. De Smidt and $E$. Wever, Eds., The corporate firm in a changing world economy. London: Routledge, pp. 21-54.

KEMPER, N.J. AND P.H. PELLENBARG (1991), Bedrijfsverplaatsingen in Nederland. Econonisch Statistische Berichten 3798, pp. 249-252.

KEMPER, N.J. (1992), Geboorte en sterfte van bedrijven. Geografie, december, pp. 3438.

KIMBERLY, J.R. (1980), Initiation, innovation, and institutionalization in the creation process. In: J.R. Kimberly and R.H. Miles, Eds., The organizational life cycle. San Fransisco: Jossey-Bass, pp. 18-43.

KLEINKNECHT, A. AND T. POOT (1992), Do regions matter for R\&D? Regonal Studies 26, pp. 221-232.

KOBERG, C.S. (1987), Resource scarcity, environmental uncertainty, and adaptive 
organizational behaviour Academy of Management Journal 30, pp 798-807 KOSTERMAN, R AND R HENTENAAR (1992), Wat er mis ging bij Volmac WSG Financieel Economısch Magazıne, 18 April, pp 8-12

KOTABE, M AND M R. CZINKOTA (1992), State government promotion of manufacturing exports a gap analysis Journal of International Business Studies 23 (fourth quarter), pp. 637-658

KRUMME, G (1969a), Towards a geography of enterprise Economic Geography 45, Pp 30-40

KRUMME, G (1969b), Notes on industrial adjustment patterns in industrial geography Geografiska Annaler 51B, pp 15-19

LAMBOOY, J G (1976), Verstedelıjkıngsnota en agglomeratie-voordelen Economisch Statistische Berichten, 31 March pp 318-322

LAMBOOY, JG (1984), The regional ecology of technological change. In JG. Lambooy, Ed, New Spatial Dynamics and Economic Cnses, Tampere IRPA Yearbook Tampere Finnpublıshers, pp 63-76

LAMBOOY, JG AND J H HUIZINGA (1977), Het nieuwe westen des lands balans van 20 jaar ontwikkeling Intermediair, pp 1-7

LAMBOOY, J G AND MANSHANDEN (1992), De mythe van de grote stad als motor van de economie Economisch-Statistische Benchten, 28 October, pp 1045-1050

LAWRENCE, P R AND J W LORSCH (1967), Organization and environment Boston graduate School of Business Administration, Harvard University

LAZONIC, W (1989), Organizational integration in three industrial revolutions In A Heertje and M Perlman, Fds, Evolving technology and market structure studies in Schumpeterian econo0mics Ann Harbor The University of Michigan Press, pp 77-98

LE HERON, R (1990), Goodman Fielder Wattie good food world-wide? Internationalization and performance In $\mathrm{M}$ De Smidt and $\mathrm{E}$ Wever, Eds, The corporate firm in a changing world economy London Routledge, pp 100-119

LEVINTHAL, A (1991), Random walks and organizatıonal mortality Admınıstratıve Science Quarterly 36, PP 397-420

LINCOLN, J R, M HANADA AND J OLSON (1981), Cultural onentations and individual reactions to organizations a study of employees of Japanese-owned firms Administative Science Quarterly 26, pp 93-115

LINGE, G J R (1992), Just-ın-tıme more or less flexible? Economic Geography 68, pp 316-332

LLOYD, P E AND P. DICKEN (1982), Industrial change local manufacturing firms in Manchester and Merseyside, Inner Cities Research Programme 6, Department of the Environment, London

LOEVE, A (1989), Buitenlandse ondernemingen in regionaal perspectief Amsterdam/Utrecht Netherlands Geographical Studies 84

LONSDALE, R E AND H L SEYLER (1979), Eds, Nonmetropolitan industralization. Washington DC Winston

LOUTER, PJ (1991), Spatial variations in economic potentials in manufacturing industries in the Netherlands Rotterdam EGI-onderzoekspublicaties

LOUTER, P J (1991), Ruımtelıje varıaties in de vertegenwoordiging van jonge bedrijvigheid In D-J Kamann and P Rietveld, Nieuwe ideeen in Nederlands ruımtelık onderzoek. Groningen/Amsterdam Regional Science Association 
Nederland, pp. 177-206.

LOUTER, P.J. (1992), Economische structuurverandering en regionale specialisatie.

Rotterdam: EGI-Onderzoekspublikaties 92-4, Erasmus Universiteit Rotterdam.

MACHIELSE, C., P.A. DE RUIJTER, H.A. VETHMAN (1990), Relatiepatronen van bedrijven in het noorden. Een empirisch onderzoek naar netwerkvorming van de industrie, het transport, de groothandel en de zakelijke diensten in het Noorden. Delft: INRO-TNO.

MALECKI, E.J. (1991), Technology and economic development; the dynamics of local, regional and national change. New York: Longman.

MALECKI, E.J. AND S.L. BRADBURY (1992), R\&D facilities and professional labour: labour force dynamics in high technology. Regional Studies 26, pp. 123-136.

MALECKI, E.J. AND P. NIJKAMP (1988), Technology and regional development: some thoughts on policy. Environment and Planning $C$ : Government and policy 6, pp. 383-399.

MARCH, J.G. AND H.A. SIMON (1958), Organizations. New York: Wiley.

MARSHALL, J.N. (1982), Linkages between manufacturing industry and business services. Environment and Planning A 14, p. 1523-1540.

MARTIN, R. (1989), The growth and and geographical anatomy of venture capitalism in the United Kingdom. Regional Studies 23, pp. 9-18.

MASO, I. (1987), Kwalitatief onderzoek. Meppel: Boom.

MASON, C.M. AND R.T. HARRISON (1985), The geography of small firms in the UK: towards a research agenda. Progress in Human Geography 9, pp. 1-37.

MEYER, J.W. AND B. ROWAN (1977), Institutionalized organizations:formal structure as myth and ceremony. American Journal of Sociology 83, pp. 340-363.

MILES, R.E., C.C. SNOW AND J. PFEFFER (1974), Organization-environment: concepts and issues. Industrial Relations 13, pp. 244-264.

MILLER, R. AND M. COTÉ (1985), Growing the next Silicon Valley. Harvard Business Review 63, pp. 114-123.

MINTZBERG, H. (1983), Structures in fives: designing effective organizations. Englewood Cliffs, N.J.: Prentice-Hall.

MITCHELL, J.C. (1983), Case and situation analysis. Sociological review 31, pp. 187211.

MOLLE, W.T.M. (1991), Cultuur en cohesie in de EG. In: D.-J. Kamann and P. Rietveld, Nieuwe ideeën in Nederlands ruimtelijk onderzoek. Groningen/Amsterdam: Regional Science Association Nederland, pp. 11-8.

MORGAN, G. (1988), Riding the waves of change: developing managerial competence for a turbulent world. San Fransisco: Jossey-Bass Publishers.

MORGAN, G. (1992), Proactive management. In: D. Mercerer, Ed., Managing the external environment: a strategic perspective. London: Sage, pp. 24-37.

MOULAERT, F., Y. CHIKHAOUI AND F. DJELLAL (19991), Locational behaviour of French high-tech consultancy firms. International Journal of Urban and Regional Research 15, pp. 5-23

MYRDAL, G. (1957), Economic theory and underdeveloped regions. New York: Harper and Row.

McCLELLAND, D.C. (1961), The achieving society. Princetown: New Jersey.

McDERMOTT, P. AND M. TAYLOR (1982), Industrial Organisation and Location. Cambridge: Cambridge University Press. 
McDERMOTT, P. (1974), Market linkage and spatial monopoly in New Zealand manufacturing. New Zealand Geographer 30, pp. 1-17.

McNEE, R.B. (1960), Towards a more humanistic geography: the geography of enterprise. Tijdschrift voor Economische en Sociale Geografie 51, 201-206.

NELSON, R.R. AND S.G. WINTER (1982), An evolutionary theory of economic change. Cambridge, Mass.: Harvard Univerity.

NEWSWEEK (2-7-1990), Help wanted.

NIJKAMP, P AND E.J. DAVELAAR (1989), Stedelijke agglomeraties en de generatie en diffusie van innovaties. Lezing gehouden tijdens de aio-cursus Ruimtelijke Economie, april.

NRC HANDELSBLAD (29-5-1991), Oprichter Infotheek wijt verlies aan eigen blunders.

NRC HANDELSBLAD (25-6-1991a), Rood Testhouse duikt in rode cijfers.

NRC HANDELSBLAD (25-6-1991b), Chiptester vecht tegen onderbezetting: diversificatie helpt Rood Testhouse nog niet van kwetsbaarheid af.

NRC HANDELSBLAD (31-10-1991), Mister Infotheek is spoor bijster na verlies fortuin.

OAKEY, R. (1984), High technology small firms. New York: St Martin's Press.

OAKEY, R.P. (1985), High technology industry and agglomeration economies. In: P. Hall and A. Markusen (eds.), Silicon Landscapes. Boston: Allen and Unwin.

OAKEY, R., R. ROTHWELL and S. COOPER (1988), The management of innovation in high-technology small firms. Innovation and regional development in Britain and the United States. London: Pinter Publishers.

OAKEY, R.P. AND S.Y. COOPER (1989), High technology industry, agglomeration and the potential for peripherally sited small firms. Regional Studies, vol. 23, pp. 347-360.

OAKEY, R.P. AND P.N. O'FARRELL (1992), The regional extent of cumputer numerically controlled (CNC) machine tool adoption and post adoption success in small British mechanical engineering firms. Regional Studies 26, Pp. 163-175.

O'FARRELL, P.N. AND B. O'LOUGHLIN (1981), The impact of new industry enterprises in Ireland: an analysis of service linkages. Regional Studies 15, pp. 439-456.

O'FARRELL, P.N. AND D.M.W.N. HITCHENS (1987), The relative competitiveness and performance of small manufacturing firms in Scotland and the mid-west of lreland: an analysis of matched pairs. Regional Studies 22, pp. 399-416.

O'FARRELL, P..N. AND D.M. HITCHENS (1990), Producer services and regional development: key conceptual issues of taxonomy and quality measurement. Regional Studies 24, pp. 163-171.

PAIJENS, R.J.M. (1989), Wie bepaalt de richting van de onderneming? Lezing Lunterencongres: Produktie, kern van concurrerende kracht. 24 and 25 October.

PARTHASARTHY, R. AND S. PRAKASH SETHI (1992), The impact of flexible automation on business strategy and organizational structure. Academi of Management Review 17, pp. pp. 86-111.

PATRICK, H. AND T. ROHLEN (1987), Small-scale family enterprises. In: Inoguchi and D.I. Okimoto, Eds., The political economy of Japan: the changing international context. Stanford: Stanford University Press.

PENROSE, E.T. (1959), The theory of the growth of the firm. New York: Wiley. 
PELLENBARG, P.H. (1985), Bedrijfslokatie en ruimtelijke cognitie. Groningen: PhDthesis, Sociaal Geografische Reeks, no. 33, RUG.

PELLENBARG, P.H. AND H. ZALME (1985), Informatieen vernieuwing. Een geografische analyse van de rol van vakbeurzen en vakbladen bij innovatieprocessen in het bedrijfsleven. Groningen: RUG.

PERROUX, F. (1955), Note sur la notion de pôle de croissance. Economie Appliquée 8, Pp. 302-320; translation in D.L. McKee, R.D. Dean, and W. H. Leakey, Eds. (1990), Regional economics: theory and practice. New York: The Free Press, pp. 93-135.

PERROUX, F. (1988), The pole of development's new place in a general theory of economic activity. In: B. Higgins and D.J. Savoie, Regional economic development: essays in honour of François Perroux. Boston: Unwin Hyman, pp. 48-76.

PERROW, C (1961), Goals in complex organizations. American Sociological Review 26, Pp. 854-865.

PERRY, R., K. DEAN AND B. BROWN (1986), Counterurbanization: international case studies of socio-economic change in rural areas. Norwich: Geobooks.

PETERS, I.J. (1990), Small business growth: spatial and non-spatial aspects of development. Southampton: Unpublished Ph.D. thesis, Department of Geography, Southampton University.

PETIT, T.A. (1967), A behavioural theory of management. Journal of Academic Management, December, pp. 341-350.

PFEFFER, J. (1972), Interorganizational influence and managerial attitudes. Academy of Management Journal 15, pp. 317-330.

PFEFFER, J. (1973), Size, composition and function of hospital boards of directors: a study of organization-environment linkage. Administrative Science Quarterly 18, pp. 349-364.

PFEFFER, J. (1981), Power in organizations. Marshfield, MA: Pitman.

PFEFFER, J. (1982), Organizations and organization theory. London: Pitman.

PFEFFER, J. AND H. LEBLICI (1973), The effect of competition on some dimensions of organizational structure. Social forces 55, pp. 268-279.

PFEFFER, J. AND G.R. SALANCIK (1978), The external control of organizations: a resource dependence perspective. New York: Harper and Row.

PIORE, M.C. AND C.F. SABEL (1984), The second industrial divide: possibilities for prosperity. New York: Basic Books.

PLATT, J. (1988), What can case studies do? In: R.G. Burgess, Ed., Studies in qualitative methodology: conducting qualitative research. London: $A$ research Annual, JAI Press, vol. 1, pp. 1-23

POLLARD, S. (1981), Peaceful Conquest. Oxford: Oxford University Press.

PORTER, M.E. (1985), Competitive advantage. New York: The Free Press.

PORTER, M.E. (1990a), The Competitive Advantage of Nations. New York: Free Press.

PORTER, M.E. (1990b), The competitive advantage of nations. Harvard Business Review, March-April, pp. 73-93.

PRAAT, H. (1992), Concumeren of samenwerken: de concurrentiepositie van de Limburgse toeleveringsindustrie. Lezing voor de bijeenkomst 'Toeleveren en samenwerken' Roermond, 27 april.

PRAKKEN, B. (1987), Turbulentiestrategieën. Bedrijfskunde 59, pp. 68-77.

PRATTEN, C. (1991), The competitiveness of small firms. Cambridge: Cambridge 
University Press

PRED, A R (1966), The spatial dynamics of U S urban-ındustrial growth 188-1914 interpretive and theoretical essays Cambridge, Mass MIT Press

PRED, A R (1967), Behaviour and location foundations for a geographic and dynamic location theory, part I Lund Studies in Geogrpahy, Series B, 27

PRED, A R (1974), Urban growth and the circulation of information, 1790-1840 Cambridge, Mass Harvard University Press.

PRED, A R (1980), Urban growth and city systems in the United states, 1840-60 Cambridge, Mass Harvard University Press

REES, J (1978), On the spatial spread and oligopolistic behaviour of large rubber companies Geoforum 9, pp 319-330

REICH, R B (1989), The quiet path to technological preeminence Scientific American 261, pp 19-25

REICH, RB (1991), The work of nations preparing ourselves for the $21^{\text {st }}$-century capitalism New York Alfred A Knopf

RENNER, G T (1947), Geography of industrial localization Economic Geography 23, PP 167-198

RICHARDSON, H W (1973), Regional Growth Theory London Macmillan

ROTHWELL, R AND W ZEGVELD (1982), Innovation and small and medium sized firms Lodon Frances Pinter

SABEL, C (1989), Flexible specialization and the re-emergence of regional economies In $P$ Hirst and $J$ Zeitlin, Eds, Reversing industrial decline? Industrial structure and policy in Britain and her competitors Oxford Berg, pp 17-70

SAXENIAN, A (1981), Silicon chips and spatial structure the industrial basis of urbanization in Santa Clara County, Californa Berkeley Workıng Paper 345, University of Calıfornia, Institute of Urban and Regional Development

SCHOENBERGER, E (1988), From fordism to flexible accumulation technology, competitive strategys and international location Environment and Planning D Society and Space

SCOTT, A J (1983), Industrial organisation and the logic of intrametropolitan location 1 theoretical considerations Economic Geography, vol 59, pp 233-250

SCOTT, A J (1988a), New industrial spaces Flexible production organization and regional development in North America and Western Europe London Pion Limited

SCOTT, A J (1988b), Flexible production systems and regional development the nse of new industrial spaces in North America and Western Europe International Journal of Urban and Regional Research 12, pp 171-186

SCOTT, A J AND DP ANGEL (1987), The US semiconductor industry a locational analysis In Environment and Planning A 19, pp 875-912

SCOTT, AJ AND M STORPER (1987), High technology industry and regional development a theoretical critique and reconstuction International Social Science Journal 112, pp 215-232

SELZNICK, P (1957), Leadershıp in Administration Evanston, IL Row, Peterson

SITTER, LU (1987), Het flexiebele bedrıf integrale aanpak van flexibuliteit, beheersbaarheid, kwaliteit van de arbeid, produkhe-automatisering Deventer Kluwer

SMALING, A AND I MASO (1990), Objectiviteit in kwalıtatıef onderzoek In I. Maso 
and A. Smaling, Eds., Objectiviteit in Kwalitatief Onderzoek. Meppel: Boom, pp. 13-29.

SIMON, H.A. (1957), Administative behaviour. New York: Theatre Press.

SMIDT, M. DE (1988), Ruimtelijke spreiding van bedrijvigheid in Nederland. Geografisch Tijdschrift 12, pp. 117-127.

SMIDT, M. DE (1991), Segmentering van de stedelijke arbeidsmarkt: theoretische perspectieven. In: O.A.L.C. Atzema, M. Hessels and $\mathrm{H}_{\text {, }}$ Zondag, Eds., De werkende stad: aspecten van de grootstedelijke arbeidsmarkt. Utrecht: Stedelijke Netwerken.

SMIDT, M. DE (1990), Philips: a global electronics firm restructures its home base. In: M. De Smidt and E. Wever, Eds., The corporate firm in a changing world economy. London: Routledge, pp. 55-76.

SMIDT, M. DE AND E. WEVER (1986), Nieuwe bedrijven in West-Europa. Geografisch Tijdschrift 20, pp. 245-252.

SMIDT, M. DE AND E. WEVER (1990a), An industrial geography of the Netherlands: an international perspective. London: Routledge.

SMIDT, M. DE AND E. WEVER (1990b), Firms, strategy and changing environments. In: M. De Smidt and E. Wever, Eds., The corporate firm in a changing world economy. London: Routledge, pp. 1-20.

SMITH, D.M. (1966), A theoretical framework for geographical studies of industrial location. Economic Geography 42, pp. 95-113.

SMITH, D.M. (1987), Neoclassical location theory. In: W.F. Lever, Ed., Industrial change in the United Kingdom. Harlow: Longman, pp. 23-37.

STARBUCK, W. (1976), Organizations and their environments. In: M. Dunette, Ed., Handbook of organizational and industrial psychology. Chicago, Ill.: Rand McNally, Pp. 1069-1123.

STAW, B.M. AND E. SZWAJKOWSKI (1975), The scarcity-munificence component of organizational environments and the commission of illegal acts. Administrative Science Quarterly 20, Pp. 345-354.

STEED, G. (1971a), Forms of corporate environmental adaptation. Tijdschrift voor Economische en Sociale Geografie 62, pp. 90-94.

STEED, G. (1971b), Plant adaptation, firm environments and locational analysis. The Professional Geographer 13, pp. 324-327.

STEED, G. (1971c), The Northern Ireland linen complex, 1960-1970. Annals of the Assciaion of American Geographers 64, pp. 397-408.

STIGLITZ, J.E. (1987), Learning to learn, localized learning and technological progress. In: P. Dasgupta and P. Stoneman, Eds., Economic policy and technological prformance. Cambridge: Cambridge University Press.

STRAUSS, A. AND J. CORBIN (1990), Basics of qualitative research: grounded theory procedures and techniques. London: Sage.

STREIT, M.E. (1969), Spatial associations and and economic linkages between industries. Journal of Regional Science 9, pp. 177-189.

STOREY, D. (1982), Entrepreneurship and the new firm. London: Croom Helm.

STORPER, M. (1992), The limits to globalization: technology districts and international trade. Economic Geography 68, pp. 60-93.

STORPER, M. AND R. WALKER (1989), The capitalist imperative. Territory, technology and industrial growth. Oxford: Basil Blackwell 
SWANBORN, PG (1990) Objectıviteit: een poging tot duidelıkheid. In: I. Maso and A. Smaling, Eds, Objectıviteit in Kwalitatief Onderzoek. Meppel Boom, pp. 5074.

TAYLOR, M J. (1975), Organizational growth, spatial interaction and location decision makıng. Regional Studies 9, pp 313-323.

TAYLOR, M J (1986), The product cycle acritique. Environment and Planning A 18. Pp. $751-761$

TAYLOR, M.J AND N. THRIFT (1983), Busıness organization, segmentation and Iocation Regional Studies 17, pp 445-465.

TERREBY, S. (1968), The evolution of organizational environments. Administrative Science Quarterly 13, pp. 590-613.

THOMPSON, C. (1989), The geography of venture capital. Progress in Human Geography 13, pp. 62-98.

THOMPSON, J D. (1967), Organizations in action. New York· McGraw Hill.

THOMPSON, J D. AND W. MCEWEN, (1958), Organizational goals and environment: goalsetting as an interaction process. American Sociological Review 23, pp 23-31.

THOSS, R. (1984), Potentialfactoren als Chance selbstverantworteter Entwicklung der Regionen. Informationen zur Raumentwicklung, Heft $1 / 2$

TNO, (1992), De vitaliteit van het Nederlandse bedrufsleven ontwikkelingen in sectoren, regio's en steden 1982-1991.

TOWNROE, P.M. (1975), Branch plants and regional development. Town Planning Review 46, Pp. 47-62.

TOWNROE, PM. (1974), Post-move stability and the location decision In. F.E I. Hamilton, Ed., Spatial perspectives on industrial organization and decision making. Chisester Wiley, pp. 287-308.

TROUW (5-11-1991), Infotheek had er nog kunnen zijn.

VAESSEN, P (1986), Nieuwe bednjven in het urbaan rurale continuum. Nijmegen: doctoraalscriptie, Katholieke Universiteit Nijmegen.

VAESSEN, P (1989), Bedrijf, regio en succes een micro-onderzoek. Nijmegen: Vakgroep Sociale en Economische Geografie, Katholiek Universiteit Nijmegen.

VAESSEN, P. AND E. WEVER (1986), Stedelıjkheid en startersinitiatief Stedebouw en Volkshuisvesting 67, pP 266-272.

VAESSEN, P. AND E. Wever (1990), Bedriff en omgeving. De betekenis van het produktiemulieu voor snelgroeiende bedrijven: 5 case studies. Assen: Van Gorcum.

VALERY, N. (1987), Factory of the future. The Economist, May 30, pp. 3-18.

VERNON, R. (1966), International investment and international trade in the product cycle. Quarterly Journal of Economics, 2, pp. 190-207.

VOûTE, A. (1991), Aandelen voor werknemers motıvatıe door partıcıpatıe Deventer: Kluwer.

VNO AND AMRO (1988), Faal- en slaagfactoren van startende ondernemingen. No publication data.

DE VOLKSKRANT (27-6-1992), Top Rood Testhouse onverwacht vervangen.

DE VOLKSKRANT (29-6-1991), Dit jaar nog verlies bij verse aankopen Infotheek.

VVK (1983 - 1992), Het bedrıfssleven in Nederland. Enquete Regionaal BedrıfssOntwıkkeling (ERBO). Woerden. Vereniging van Kamers van Koophandel en Fabrieken in Nederland. 
WALKER, R. (1985), Technological determination and determinism: industrial growth and location. In: M. Castells, Ed., High technology, space and society. London: Sage, pp. 226-64.

WALKER, R. (1988), The geographical organization of production-systems. Environment and Planning: Society and Space 6, pp. 377-408.

WALKER, R. (1989), A requiem for corporate geography: new directions in industrial organization, the production of place and uneven development. Geografiska Annaler 71B, 1, pp. 43-68.

WATTS, H.D. (1978), Inter-organisational relations and the location of industy. Regional Studies 12, pp. 215-225.

WATTS, H.D. (1980a), The large industrial enterprise. London: Croom Helm.

WATTS, H.D. (1980b), Conflict and collusion in the British sugar industry, 1924-28. Journal of Historical Geography 6, pp. 291-314.

WATTS, H.D. (1981), The branch-plant economy: a study of external control. London: Longman.

WEICK, K.E. (1979), The social psychology of organizing. Reading, MA: AddisonWesley.

WELCH, L.S. AND F. WIEDERSHEIM-PAUL (1981), Domestic expansion: internationalization at home. Reprint series 1981/2: Department of Business Administration, University of Uppsala.

WESTHEAD, P. AND S. BIRLEY (1992), Environments for business derigistrations in the United Kingdom, 1987-1990. Paper at the conference, small firm 1992.

WESTER, F. (1987), Strategieën voor Kwalitatief Onderzoek. Muiderberg: Coutinho.

WEVER, E. (1984), Nieuwe bedrijven in Nederland. Assen: Van Gorcum.

WEVER, E. (1985), Regionaal ekonomisch perspektief. Een literatuurverkenning naar het ruimtelijk patroon van de bedrijvigheid in Nederland. Nijmegen: Vakgroep Ekonomische Geografie, KUN.

WEVER, E. (1986), New firm formation in the Netherlands. In: D. Keeble AND E. Wever (eds.), New firms and regional development in Europe. London: Croom Helm, PP. 54-74.

WEVER, E. (1990), ccFriesland: Spatial dynamics within a dynamic company. In: $M$. De Smidt and E. Wever, Eds., The corporate firm in a changing world economy. London: Routledge, pp. 120-144.

WEVER, E. (1991), Bedrijf en omgeving, een ongemakkelijke relatie. Economisch Statistische Berichten 23 October, pp. 1072-1076.

WHETTEN, D.A. (1981), Organizational responses to scarcity: exploring the obstacles to innovative approaches to retrenchment in education. Educational Administration Quarterly 17, pp. 80-97.

WIEDERSHEIM-PAUL, F., H.C. OLSON, L.S. WELCH (1978), Pre-export activity: the first step in internaionalization. Journal of International Business Studies 9. pp. 47-58.

WILLIAMSON, O. (1975), Markets and hierarchies. New York: Free Press.

YUCHTMAN, E. AND S. SEASHORE (1967), A system resource approach to organizational effectiveness. American Sociological Review 32, pp. 891-903.

ZUCKER, L. (1983), Organizations as institutions. In: S. Bachrach, Ed., Research in the sociology of organizations. Greenwich, Conn.: JAI Press.

ZWAN, A. VAN DER (1990), Koplopers en achterblijvers: effectiviteit in beleid en 
management. Baarn: Anthos. 


\section{The sample firms one by one}

\section{Aalberts Industries plc}

Aalberts Industries plc is the holding company of, at present, 11 industrial enterprises, employing a total of about 1000 employees. In close consultation with the customer, these factories manufacture tailor-made products, mostly from high-quality aluminium alloys, advanced kinds of steel alloys and synthetic materials. The group has customers spread over a large number of business sectors, including medical equipment, aeronautical engineering, aerospace, computer peripherals, telecommunications, aluminium processing, precision engineering, and many others.

The foundation of the Aalberts Industries Group was laid in 1975, when Mr. J. Aalberts started a company named Mifa Ltd. (Mini Fabrication) in Reuver, a village some $12 \mathrm{~km}$ from Venlo. This business manufacturered and assembled precision engineering components by way of a refined extrusion technique which had not yet been applied in Europe. The beginning was very difficult, even though Aalberts was on familiar ground and he knew many potential customers. When the early development problems had been overcome, the very accurate production processes drew attention of big enterprises such as Philips, IBM, Siemens and Océ-van der Grinten. From that point, in 1977, the company started its impressive advance. The industrial premises in Reuver becomes too small, and Mifa moved to an industrial estate in Venlo. By 1983, the Aalberts Industries Group had a trunover of 14 million guilders and 133 employees. In the four years prior to the start of the present research project, sales figures rose by an average of 40 per cent per year (from 14 million guilders in 1984 to 48 million in 1987). The number of workers hired increased to 255 in 1982. These figures compare very favourably with those of the basic metal industry as a whole. Sales in this sector grew in the same period on average 5.5 per cent per year. The profits of Aalberts Industries also towered above performance figures of the metal industry as a whole. Between 1984 and 1987, after-tax profit amounted on average to 5.7 per cent of sales for Aalberts Industries, in comparison to 3.7 per cent for the aggregate metal industry.

Since 1987, Aalberts Industries has been listed on the Amsterdam Stock Exchange. In 1988, the company won the FEM/PA medal, a distinguished prize for innovative entrepreneurship. After 1987 the growth and success of the company did not decrease. It took over one company after the other. In 1989, Aalberts relocated its holding seat from Venlo to Utrecht, though this involved only a limited number of 
staff members (less than ten). Furthermore, up to now, Aalberts has hardly been affected by the decline in the world economy. By 1992, sales had mounted to nearly 218 million guilders, and the number of employees had risen to 1006. Net profit was 5.7 percent of net sales in 1992.

The interview was conducted on 18 February 1988, with the president and founder of the Aalberts Industries Group, Mr. J. Aalberts. Director Aalberts was born in 1940. His educational background included Technical College.

\section{Bronkhorst High Tech Ltd}

In March 1981, Mr. T. Bruggeman contacted Mr W. Jouwsma with the proposal to start a business company together. The idea was to integrate the different pieces of equipment required for measuring gas flow, such as sensors, valves and accompanying electronics, into one handy instrument. Jouwsma thought that this would pay off, and thus a new firm was created, named Bronkhorst High Tech (BHT). BHT is engaged in the production of electronic flowmeters. The two men started their enterprise in Vorden, a small village situated in a rural landscape on the eastern outskirts of the country, a region known as de Achterhoek.

BHT sells its flow meters to the semiconductor industry and chemical laboratories, among others. Since actual production got off the ground, BHT has been operating very profitably. Soon, the industrial estate in Bronkhorst became too small and BHT moved to Ruurlo, a small village some ten kilometers away. To this day the enterprise has remained in this place. In 1987, sales amounted to approximately 7.5 million guilders. Between 1984 and 1987, tumover expanded at a rate of 24.0 per cent per year. Growth figures of the industrial class to which Bronkhorst belongs (i.e. optical, instrument and other industry) only came to 6.6 per cent per year in this period. The number of employees at Bronkhorst increased from 16 in 1984 to 37 in 1987. Profit after taxes has remained at a high and stable level of 10 per cent of sales. In 1987, Bronkhorst High Tech received the King Willem I plaquet for its achievements, a distinguished award for young entrepreneurship granted every two years to an excelling young business.

In recent years, sales growth gradually slowed down to seven per cent in 1992. Of course, this figure is still higher than the annual 4.5 per cent increase in the optical and instrument industry as a whole in this period. Between 1987 and 1992, the number of employees hired increased at a slow but steady pace, i.e. from 41 to 50 . However, notwithstanding the slower growth, BHT remains very profitable. Up to and including 1992, a hard year for the national economy when many firms went bankrupt, the net profit of BHT remained at the very high level of 10 per cent of sales.

Bronkhorst High Tech was visited twice within the scope of the present research, once in early 1988 and again in January 1990. Both conversations were with Mr. J. Jouwsma, who is the Technical Director of BHT. Jouwsma was born in 1943. He attended the Technical University of Eindhoven, where he graduated in electrical engineering as well as in business administration. 
Casolith Sheets Ltd. was established in 1920 in Leeuwarden under the name of Dutch Casolith Works. Casolith started manufacturing products from natural milk protein, particularly synthetic hom. The company supplied this product mainly to the button industry. Apart from this, in the course of time Casolith increasingly buckled down to the production of cast acrylic sheets, known by the brand name Casocryl. Casocryl is a so-called PMMA product in the field of polymer chemistry. Casocryl sheets serve as semimanufactured supply products for the automotive industry, sanitary industry, illuminated advertising industry, the construction sector and others. Competing brands of Casocryl include, for example, Perspex (ICI, Great Britain) and Plexiglass (Rohm, Germany). In the mid-1970s, Casolith reached the largest size in its life up to now, i.e about 200 employees. However, soon thereafter, the company got in serious trouble, and heavy losses were incurred. In 1978, the Regional Development Corporation for the northern provinces (the NOM) decided to take over Casolith. The family managers left the firm. In 1980, a person from the NOM's own ranks came forward to take the lead over the firm. This man was Mr. E. Jansen. Jansen succeeded in making Casolith Sheets profitable again within two years. From 1983 onward, the company grew quite successfully. Between 1984 and 1987, the average annual increase of sales was 21.3 per cent (from 20 mullion guilders in 1983 to 46 million in 1987). The comparable figure for the chemical industry as a whole amounts to a mere 2.8 per cent. Employment expanded from 82 staff workers in 1983 to 128 in 1987. Annual net profit was on average 4.7 per cent from sales and 5.8 per cent of total assets in this period. For the chemical industry as a whole, these figures were 3.2 and 5.2 per cent respectively. By 1987, the company had grown into the fifth largest (out of about 20) European producer of acrylic sheets. Its share on the European market increased from two per cent in 1980 to seven per cent in 1987.

In 1986, Casolith applied for quotation on the Amsterdam Stock Exchange. However, the listing did not last very long. In Spring 1987, Jansen decided to go along with a take-over bid by the French state enterprise Chemie de France (CdF). $\mathrm{CdF}$, which changed its name in 1988 into Orkem, merged Casolith with two other PMMA manufacturers within the Orkem Group. Director Jansen acquired general directorship over this new PMMA group, which is the biggest in Europe. Thus, he left Casolith in November 1988 and moved to Paris, where the headquarters of Orkem is located. Mr. J. Spoelstra, formerly Casolith's production manager, was appointed as Jansen's successor. In 1991 Casolith Sheets merged into Atochem Holland Holding Ltd. in Amsterdam. At that time, the name of the company changed into Atochem Leeuwarden Ltd. In October 1991, director J. Spoelstra also left the company. He was succeeded by Mr. F.M. De Haas.

The take-over by Orkem ${ }^{1}$ has far reaching organizational consequences for Casolith Sheets. The integration with the other two PMMA manufacturers required a reorganization of the company and that was not without some difficulties. Nevertheless, Casolith was able to maintain its rate of growth. Between 1988 and 1990, sales on average increasd by 23.7 per cent and employment by 5.2 per cent per year. Average annual growth in the chemical industry as a whole was much slower: 4.1 and 2.6 per cent respectively. Furthermore, the annual report for 1991 mentioned that even 
though the PMMA market was in decline that year, which also affected Casolith's sister companies, Casolith itself was able to increase its sales by a modest 1.5 per cent. Average profitability remained about 4.5 per cent from sales, which is below the Chemistry sector's average, which amounted to 7.0 per cent between 1988 and 1990 .

Just like Bronkhorst High Tech, Casolith Sheets was also visited twice during the research. The first interview was conducted in 1988 with Mr. E. Jansen. Jansen was born in 1948. When he finished his secondary education he went on to study economics, first at Rotterdam's Erasmus University and later at the University of Groningen. The second interview took place in early 1990 with Mr. J. Spoelstra. Spoelstra was born in 1956 in a small village in the province of Friesland. Spoelstra completed the Technical College in Groningen.

\section{System House Comdes Ltd}

The year was 1978 when Mr P. Quekel approached Mr. W. Kragtwijk with the proposal to start a new software company. Their basic idea was to incorporate more material knowledge into software packages than was customary in those days in the world of software engineering. The two men sought to achieve this by specializing in a limited number of market segments, i.e. financial services and the legal and healthcare sectors. Furthermore, in order to stand out in the branch of software engineering, soon after start-up Quekel and Kragtwijk decided to take on automation projects at fixed prices instead of hourly wages. This approach led to very rapid growth. Within five or six years, the company has become one of the largest software engineering companies in the Netherlands. Between 1984 and 1987, the growth rate on average amounted to 60.5 per cent per year. In 1987, the turnover amounted to 35.6 million guilders, which eventually increased to 51.6 million in 1988 . Employment expanded from a staff of 57 in 1984 to 161 in 1987 (1988: 175). This represents an average annual increase of 41.6 per cent. These figures are far in excess of the achievements in the business services sector as a whole, where growth averaged 6.6 per cent and employment increased by 10.5 per cent. Net profit of System House Comdes - 4.0 per cent of sales - towered less highly above the industry total - 3.6 per cent - between 1984 and 1987 than the growth figures. Anyway, Comdes took a fifth and an eighth place in the top 25 list of fastest-growing small companies in 1986 and 1987 (Intermagazine, November 1986 and November 1987).

However, already in 1987 things started to go wrong for Comdes, as the company suddenly suffered a loss of 1.9 million guilders. In 1988 this loss amounted to 7.6 million guilders. One year later, in 1989, System House Comdes was taken over by one of its shareholders, namely Modalfa Ltd., a private investment group from Amsterdam. Quekel and Kragtwijk were bought out and they left the company in April 1989. Modalfa appointed a new general manager, Mr. Th. Vissers, who had to make the company profitable again. In a radical reorganization, Vissers slashed the activities of Comdes drastically. As a consequence, sales fell to about 8 million guilders and personnel to 88 by the end of 1990 . Nevertheless, it was not possible to make the company profitable again in 1989 and 1990, although the losses were cut back from 24.2 million guilders in 1989 to 10.9 million in 1990. Early in 1991, Modalfa 
sold Comdes to the British information technology company ICL. In the meantime Vissers left the Comdes Group.

Conversations were held made with Mr. W. Kragtwijk and Mr. Th. Vissers, in the early Spring of 1988 and 1990 respectively. Kragtwijk was born in the beginning of the 1950s. In the period when he and Quekel led the Comdes Group, Kragtwijk fulfilled the function of technical director, whereas Quekel was the commercial man. Kragtwijk's educational background was lower general secondary education, supplemented with evening classes in the field of computerization. Vissers was born in 1942. Vissers had secondary school (i.e. HBS) followed by several marketing courses, including NIMA-A and NIMA-B.

\section{Database Consultancy Investments plc}

The foundation for a group of companies which are united in the Holding company called Database Consultancy International (DCI) was laid in Amsterdam in 1980 with the establishment of Database Consultancy Europe Ltd. (DCE). The founder of the $\mathrm{DCl}$ Group is Mr. K. R. Greystoke. Database Consultancy International is mainly engaged in information management consultancy services. Areas of DCE services include information systems strategy, systems development methods and tools, information resource management and data communications. Between 1984 and 1987, the company's average annual growth rate (sales) was no less than 62.1 per cent business services as a whole grew by 6.6 per cent - whereas employment increased from 48 to 215 workers in those years. Average profit as a percentage of sales in the four-year period prior to the beginning of the research was 5.6 per cent per year; for the service sector as a whole this figure was $4.0^{2}$. The year 1988 was a good one for DCl, as sales, profits and employment kept growing. However, in 1989, only employment retained its growth momentum, as the number of workers increased from 229 in 1988 to 284 in 1989 . The rate in growth of tumover diminished to 14.4 per cent and profit even turned into a loss, albeit a small one of 689,000 guilders $(-1.3$ per cent of sales). The decline of the company increased in 1990. In that year, sales diminished by 10.1 per cent, losses rose to -4.0 per cent of sales and employment was cut back to 218 workers. Greystoke resigned as chairman of the board of directors and was succeeded by the financial director of the company, Mr. E. De Zoete. Nevertheless, in 1991 the situation worsened further, and in 1992 the company was liquidated.

The interview for this research was conducted in the Spring of 1988 with Mr. E. De Zoete. Although De Zoete is not the founder of the DCI Group, he had been engaged in the establishment of the company from the beginning. After all, he was a key figure in raising the start-up capital. During the first six years de Zoete was involved in DCI as a commissioner and adviser. In 1986, he became the company's financial manager. De Zoete was born in 1936. He had received academic education in the field of economics. 


\section{Elephant Industries Ltd}

Elephant Industries was established in Amsterdam in 1969 by its present general Director Mr. M. Brouwers. Elephant Industries manufactures dental products from precious metal and also from porcelain. They work with precious metal alloys, precious metal prosthetic devices and other similar items. Elephant Industries' products serve as input materials for prosthodontics. The company had its highest growth rates in the 1970s, according to director Brouwers. This forced the company to relocate three times in that decade. In 1970 Elephant Industries moved from Amsterdam to Zaandam. Within Zaandam the company relocated in 1974. By 1977 its business office had become too small again, and the firm moved from Zaandam to Hoorn, its present location. In the 1980s, growth slowed down somewhat, but the company remained vital. Between 1984 and 1987 turnover increased on average by 19.6 per cent per year (from 34.2 million to 48.0 million guilders). The total of Elephant's respective industry category, i.e. basic metal industry, grew on average 3.5 per cent per year in this period. Employment at Elephant Industries rose from 43 workers in 1984 to 63 in 1987. Average net profit amounted to 3.8 per cent for Elephant Industries. For basic metal industry this figure is not known; however, Elephant Industries was slightly more profitable than the entire metal industry (3.6 per cent).

After 1987, the company was able to continue both its growth and its profitability, although there was a dip in cash terms in 1989 and 1990. That was due to low prices for precious metals on the world market. Between 1988 and 1991 Elephant's annual monetary sales growth averaged 5.7 per cent (basic metal industry scored 4.0 per cent). Employment kept on growing at a steady pace from 66 employees in 1988 to 83 in 1991. The company's average net profit was 2.7 per cent per year, which was below the average of $5.0^{3}$ per cent for the metal industry as a whole.

The conversation with director Brouwers took place in the beginning of 1988 . Before Brouwers entered the labour market, he had had an advanced level of elementary education (MULO).

\section{Groenendijk plc}

Groenendijk plc was established in 1947 in Rotterdam as a transport firm by Mr. M. Groenendijk. However, significant growth did not get off the ground until 1972, when the company, in addition to providing transport services, began letting out mobile site huts. This line of business became increasingly important in the course of time. By now, Groenendijk has grown into the national market leader in the renting of temporary business accommodation. Between 1984 and 1987, the average annual sales increase was 33.7 per cent, whereas the comparable figure for other services (i.e. not including financial and banking or business services) amounted to 5.5 per cent. The number of personnel hired increased from 36 in 1984 to 62 in 1987. The company was extremely profitable. Profit as a percentage of sales was very high, averaging 25.4 per cent per year between 1984 and 1987. Measured as a percentage of total assets, the profitability of Groenendijk was 16.3 , whereas services as a whole came to only 4.0 
per cent. In 1985, the company moved to its present location in Ridderkerk, which is adjacent to Rotterdam. In 1986, Groenendijk applied for quotation on the Amsterdam Stock Exchange.

After conducting the interview in early 1988, these figures did not decline. Between 1988 and 1991 average annual sales growth came to 294 per cent, staff employed increased from 68 to 165 workers in this four-year penod. Profitability remained at the high level of 20.4 per cent of sales and 11.8 per cent of total assets. However, in 1992, Groenendıjk was affected by the latest economic recession. Turnover was still increasing, albeit at the modest rate of 5.6 per cent However, net profit dıminished by no less than 54.6 per cent. Nevertheless, profit stıll reached the considerable level of 7.1 per cent of sales and 4.5 per cent of total assets. Many firms would be happy with these figures even when the economy is booming.

The interview was conducted with director K. Groenendık, who is a son of Mr. M. Groenendijk. Groenendijk Junior was born in 1949. He became a fellow director of Groenendijk plc in the early 1970s. He received his education at the School for Business Administration and Economics.

\section{Hulo International Ltd}

Hulo International, which is located in the small village of Pannerden (Achterhoek), was established in 1916. Initially the firm was a modest engineering company catering to the needs of the brick industry in the eastern part of the Netherlands. However, in the course of time, the company developed into a modern firm in transport and logistics systems, special handling equipment, robotic manıpulators and related appliances. At the tume the interview took place, Hulo International was a member of Phoenix Megatronica plc at Oosterbeek This is a holding company of about eight smaller businesses engaged in developing systems for process control, environmental technology, measurement and test systems, and pumps. Hulo is by far the largest member of the group. As no information is avallable about the turnover of Hulo before 1985, performance figures about the year 1984 had to be left out We worked with the figures from 1985 onward. Furthermore, the annual reports of Hulo do not give absolute numbers for the company's turnover, only annual percentages of increase or decrease. In 1986 and 1987, the company's sales increased at a rate of 21.1 and 10.2 per cent respectively. This compared extremely well with the annual sales growth of 3.1 and 5.1 per cent for the machine industry in the same years. Employment statistics of Hulo, on the other hand, are avallable from 1984 onward. The number of staff employed rose from 43 in 1984 to 65 in 1987. This is an average increase of 15.7 per cent per year, whereas in the machine industry as a whole employment rose on average only 1.7 per cent per year in the same period. From the public Hulo reports, net profits are known only from 1987 onward. In 1987 this figure came to 52 per cent of total assets, which was higher than the aggregate figure for industry as a whole ( 3.8 per cent) In 1988, Hulo's turnover increased by 128 per cent, but the company's profit declined. It fell below the aggregate industry figure (Hulo, 2.4 per cent of total assets, manufacturing industry 4.0 per cent of total assets). In 1989, the turnover dıminished as well, skıddıng at a rate of 4.6 per cent Profit after 
tax turned into a loss that year, albeit a rather small one of 430,000 guilders. Employment, in contrast, increased from 60 workers in 1988 to 66 in 1989. In January 1989, Hulo International left the Phoenix Megatronica Group and became independent. Later on that year, the company was taken over by a Swedish enterprise, named MTHGruppen AB. In 1992 MTH terminated Hulo's activities in Pannerden and transferred them to Sweden. The interview we conducted took place in March 1988 with the then director Mr. Meeus.

\section{Infotheek plc}

Infotheek is the brainchild of Mr. W. Van Leenen. Van Leenen established Infotheek in 1980 in the city of Leiden. The company was primarily engaged in the computer trade. From 1983 onward, the new firm experienced a dazzling growth. Between 1983 and 1987, sales increased from 4.7 million to 100.2 million guilders, an average annual growth rate of no less than 124.9 per cent. Personnel hired increased in this period from 10 to 156. On average, the annual profit came to 5.7 per cent of sales (for the category of trade activities excluding wholesale and retail trade, this figure was 3.2 per cent). In 1986, Infotheek was listed on the Amsterdam Stock Exchange parallel market. Three years later the company took the step to the official market. By 1988, Infotheek's office had become too small. The company moved from the inner city of Leiden out to the city limits, into a prestigious new office. In that year, 1988, and the subsequent ones, 1989 and 1990, sales skyrocketed to $146.8,270.6$ and 447.4 million guilders respectively. By 1990, personnel employed had increased to 701 staff workers. However, in 1990, completely unexpectedly, the management was confronted with a financial loss, albeit not very large, of 433,000 guilders. Nevertheless, the firm did not survive the subsequent year of 1991. In a timespan of half a year it was wiped. The direct reason for Infotheek's sudden collapse, which received much attention in the press in those days, was the complete failure of the company's international expansion. This was accompanied by a number of very unfortunate large-scale foreign acquisitions.

The conversation with general director Van Leenen took place in 1988. The Infotheek director was born in 1943 in The Hague. Prior to entering the labour market, Van Leenen had studied mathematics at the University of Leiden, although he did not finish this study. In addition, he had taken a course in Higher Business Management.

\section{Intercai Ltd.}

Intercai is a vital company which supplies telematics consultancy services. The firm was founded in 1979 in the village of Beek, which is close to Maastricht. The founder of Intercai is Dr H. Kivits. In 1982, the young firm moved to its present location, Geleen. Company growth got off the ground in 1981 and got larger in 1983. Between 1984 and 1987, sales increased from 900,000 to 7.2 million guilders. The growth rate amounted on average to 71.0 per cent per year. The mean annual expansion of 
aggregate business service activities was 6.6 per cent during this time period, and pales beside the growth of Intercai. Staff employed increased from 19 workers in 1984 to 52 in 1987. Net profit was 9.7 per cent of sales on average per year in this period (cf service industry's, 3.6 per cent). Intercai successively occupied the 17th, 11th and 13th place on the annual top 25 list of fastest-growing small companies between 1986 and 1988 (Intermagazine November 1986, 1987 1988). In 1989, the company received the King Willem I plaquet, for young entrepreneurship, the award which Bronkhorst High Tech won in 1987.

Up to now, the economic recession seems to give Intercai little trouble. In the years 1990 and 1991 sales increased to 20.0 million and 24.4 million guilders respectively. The number of employees rose to 133 and 160 in these years. Net profit remained at the high level of 8.0 and 8.3 per cent of sales in 1990 and 1991 respectively (for business services as a whole these figures were 5.8 and 3.7 per cent).

Intercai Ltd. was visited twice within the context of the present research. In 1988, an interview was conducted with general director Kivits, and in 1990 an interview with staff member Mr. J. Donders. Mr. Donders was instructed by director Kivits. For the benefit of the second interview, a list of questions had been sent to Kivits. Kivits' answers to these questions were considered in the conversation with Donders.

Director Kivits was born in 1953. He studied chemical technology at the Technical University of Eindhoven, and in 1980 he took his doctorate degree in this field at the same university. At the time of the interview, Mr. J. Donders had the position of manager of the Regional Policy unit and was also responsible for the company's public relations policy. Like director Kivits, Donders was born in 1953 . He received higher vocational education prior to his work experience.

\section{Nedap plc}

The establishment of Nedap ple dates from 1929. At the end of the 1940s, the company relocated from Amsterdam to its present location in Groenlo. Initially Nedap manufactured Bakelite door/window fixtures, low and high-voltage current products such as bell transistors, bell push buttons, programme selectors, and transistors. In the 1960s, the company gradually hived off this entire production programme. In the 1970s, it buckled down to the production of electronic detection and identification systems, such as hands-free access control systems, electronic antishoplifting systems, cattle code responders, tool identification systems and, many more electronic high-tech products. From these years onward, growth occurred very rapidly, although the company was affected by the economic crises at the beginning of the 1980s. However, after a reorganization and one year, 1982, of losses, the troubles were overcome. Between 1983 and 1987, sales increased at an average annual rate of 17.0 per cent (from 28.5 million guilders in 1983 to 60.3 million in 1987). The electronics industry as a whole grew in this period at a mean rate of 5.7 per cent per year. Furthermore, Nedap saw its workforce increase from 259 to 317. Mean net result came to 5.4 per cent of sales and 7.0 per cent of total assets per year. For industrial activities as a whole, excluding the metal industry, construction, food industry and 
chemistry, these figures came to 3.1 and 2.9 per cent. For its innovative entrepreneurship, Nedap was honoured with the FEM/PA medal in 1987.

Thereafter, growth and profitability did not diminish. By 1991, sales had increased to 100.3 million guilders, and employees to 421 . Net profit remained at about 5.8 per cent of sales and 8.3 per cent of total assets. Nedap is also listed on the official Amsterdam Stock Exchange.

Nedap is run by a two-headed management team. In 1988, when the interview took place, management consisted of Mr. R Paijens and Mr. A. Westendorp. Both directors were present at the meeting. Director Paijens was born in 1924. He left the firm in 1989, when he retired. Paijens, who is the driving force behind Nedap's remarkable success, has worked at the firm for about 40 years (from 1967 onward as a director). His educational background consists of technical schooling at the secondary level (the Electrical Engineering School in Amsterdam). Mr. Westendorp has worked for Nedap since 1973, and since 1985 as a director.

\section{Rood Testhouse plc}

In 1979, a new firm called Rood Testhouse was started in a small village some $20 \mathrm{~km}$ from Zwolle. The founders were Mr. J. Bijsterbosch and Mr. W. Weishaupt. The new company was primarily engaged in providing services concerning the testing of electronic circuits. For four years prior to the beginning of the research, the company underwent remarkable growth. Sales increased from 6.7 million guiders in 1984 to 13.7 million in 1987, an average expansion of 29.1 per cent per year. Personnel hired rose from 65 to 123 in this period. Profits fluctuated between 5.2 and 12.4 per cent of sales, which is significantly higher than the profitability in the service industry as a whole, which fluctuated between 4.1 and 3.2 per cent of sales. In 1985, the company received the King Willem I plaquet for young entrepreneurship. Since 1986, the company has been quoted on the parallel market of the Amsterdam Stock Exchange.

Between 1988 and 1991, sales growth increased further from 23.1 million to 58.5 million guilders in 1991. Employment expanded to 407 workers in 1991. This growth was mainly caused by the acquisition of the German company SES Electronics GmbH (SES) on January 1, 1991. Notwithstanding these growth figures, Rood Testhouse went through a severe crisis in this period. In 1990, it suddenly found itself confronted with a financial loss of no less than 21.0 per cent of sales 9.0 million guilders). In 1991, a far-reaching reorganization was set in motion. As a result, the company's loss shrank to 400,000 guilders in 1991 (-0.01 per cent of sales). However, this was not the end of it. In June 1992, the two founders, directors Bijsterbosch and Weishaupt, were replaced by two new directors, Mr. E. Pfeilerder, who is the director of SES, and Mr. L. Van Bree. Furthermore, all Dutch activities of Rood Testhouse were closed down at the end of 1992, and the international activities of the enterprise were transferred to Singapore. By the way, the enterprise is still quoted on the parallel market of the Amsterdam Stock Exchange.

The interview was conducted with the two founders of Rood Testhouse. It took place in January 1988. Both directors were born in 1946, and both have enjoyed higher vocational education. 


\section{Waterland Efficiency Group Ltd}

The Waterland Group in Purmerend has its origin in two separate companies; an accountancy company and a system house for software engineering. Both businesses merged in 1979 into one enterprise, the Waterland Efficiency Group. In this research we concentrated primarily on the automation division of the Waterland Group. The core activities of Waterland Automation consist of developing computer software for the benefit of industrial production automation. It develops software packages to support careful planning of input materials required for ever-more complex and flexible production processes in manufacturing industry.

Waterland Automation was establish on January 1, 1978 in Amsterdam by Mr. P. Schoneveld. In the course of 1979, the software firm of Pieter Schoneveld merged with the accountancy business of his brother. The new firm, Waterland Efficiency Ltd., located in Purmerend. The automation division accounts for about 85 per cent of the total turnover of the Waterland Group, whereas the accountancy division accounts for the remaining 15 per cent. The Waterland Group has become quite successful. By 1983, the company achieved a turnover of nearly 10 million guilders. From then on, its growth accelerated. Between 1984 and 1987, sales increase on average by 34.2 per cent per year (cf aggregate business service industry, 6.6 per cent). The number of employees rose in this period from 53 to 149. Net profit came to 7.2 per cent of sales and 6.6 per cent of total assets on average per year. For the aggregate service industry, these figures are 6.6 per cent and 4.1 per cent respectively. It is not surprising that the Waterland Group appeared on the top 25 list of fastest-growing small companies in those years. In 1986 and 1987, the company took 19 th and 16 th place respectively.

However, already in 1988 the firm fell into deep trouble, as it incurred a loss of 5.9 million guilders. In the subsequent year, this did not diminish. By early 1992, Waterland was declared bankrupt.

Within the scope of this research, the Waterland Group was visited twice, once early 1988 and again two years later in January 1990. The first interview was conducted while two directors were present, the Ceneral director Mr. P. Schoneveld and the Commercial director Mr. P. Haakmeester. The second conversation was conducted with the director, Mr. Schoneveld. Director Schoneveld, a middle-aged man, has enjoyed higher vocational education at a technical college. Mr. Haakmeester was responsible for the internationalization of the company. He was the manager of Waterland International, a subsidiary of the Waterland Group, located in Belgium.

\section{Notes}

1. In 1990 Orkem became dismantled. The French Ministry of Industrial Affairs deeided to divide Orkem's activities among the oil and chemistry concerns of Société Elf Acquitaine and Total-Compagnie Francaise des Pétroles. Thereby, Orkem's PMMA production, including Casolith Sheets, was transferred to Elf.

2. This figure for the aggregate service sector is slightly different from the 3.6 per cent mentioned previously, when describing System House Comdes. For the year 1984, DCI 
only had information available on profit before tax For reasons of comparison, the sectoral figure for 1984 is also based on profit before tax instead of profit after tax.

For the year 1991, statistics on industrial subcatcgories have not become available yet. For that reason we used the profitability figure of total manufacturing industry. (Source: Statistisch Bulletin 16-4-1992). 


\section{Snelgroeiende bedrijven in ongelijke gebieden}

Hoewel welvaart vaak wordt toegeschreven aan de prestaties van afzonderlijke landen (we spreken bijvoorbeeld van rijke vs. arme landen) is de voortbrenging daarvan niet een zaak die zich primair op het nationale niveau afspeelt. Ruimtelijke wetenschappers wijzen erop dat de rijkdom van een land wordt geschapen in één of een beperkt aantal gebieden binnen dat land. Zulke regio's worden aangeduid als economische kern- of centrumgebieden. Economische groei, zo luidt de opvatting, vindt geconcentreerd in de ruimte plaats en regionale ongelijkheid is inherent aan het verschijnsel groei. De achterliggende gedachte is dat er door opeenhoping van bedrijven en voorzieningen een gunstig milieu ontstaat voor het bedrijfsleven om te vernieuwen en te groeien. Gewezen wordt dan vaak op de veelheid van universiteiten, de aanwezigheid van hoog opgeleide mensen, professionele ondersteunende diensten en toeleveranciers en een grote afzetmarkt in dergelijke kernregio's. Het belang van de kwaliteit van de plaatselijke omgeving voor de groei van de nationale economie wordt recentelijk ook buiten de vakterreinen van de ruimtelijke wetenschappen steeds meer erkend en benadrukt. In dit opzicht lijkt er sprake van een '(her)ontdekking' van de geografie.

Globaal genomen zijn, naar alle waarschijnlijkheid, de krachten die leiden tot opeenhoping van economische activiteiten inderdaad sterker dan de spreidingstendenzen in de economie, $\mathrm{zij}$ het dat er grote verschillen kunnen bestaan tussen sectoren en landen. Hoe dan ook, lang niet alle goedlopende bedrijven zijn in economische kerngebieden gevestigd. Soms tref je ze aan op de 'onmogelijkste' lokaties. Dergelijke bedrijven zijn altijd enigszins buiten de aandacht gevallen van het ruimtelijk economisch onderzoek, mede omdat het vaak om beperkte aantallen gaat. In elk geval bestaat er geen goed uitgewerkte theorie die de locatie van snelgroeiende bedrijven buiten het economisch kerngebied kan verklaren.

Het voor u liggende onderzoek stelt deze groep van snelgroeiende bedrijven op niet-centrale of perifere lokaties juist centraal. Het tracht een verklaring te vinden voor hun groei tegen de achtergrond van het veronderstelde belang van de omgeving voor het functioneren van kleine en middelgrote ondernemingen. Naar het beleid toe is de achterliggende doelstelling om te kijken wat we kunnen leren van 'sterke bedrijven in zwakke gebieden' ten behoeve van de ontwikkeling van achtergebleven gebieden.

Om dit te realiseren zijn verspreid over het gehele land 13 snelgroeiende kleine en middelgrote ondernemingen uitvoerig onderzocht met behulp van zoge- 
naamde diepte-interviews. Van deze dertien zijn er negen gevestigd buiten het westelijk kerngebied, i.e. de Randstad. Deze negen hebben we vergeleken met vier bedrijven die wel in de Randstad zijn gevestigd.

De centrale onderzoeksvraag luidt waarom sommige 'ongunstig' gelegen bedrijven niet minder succesvol zijn dan hun 'gunstig' gevestigde tegenhangers? Het proefschrift tracht die vraag in drie stappen te beantwoorden. Op de eerste plaats hebben we ons afgevraagd hoe de onderzochte bedrijven concreet reageren op de mogelijkheden en vooral de beperkingen van hun vestigingsgebied. Niet verwonderlijk, blijkt dat de ondernemingen niet passief blijven ten opzichte van hun omgeving en er op honderd en een manier op kunnen reageren. Feit blijft dat theorieën over regionale ontwikkeling met dit gegeven niet goed uit de voeten kunnen. De interacties die we tussen bedrijf en omgeving hebben kunnen waarnemen kunnen worden ingedeeld in vier categorieën. Op de eerste plaats benutten ondernemers natuurlijk de mogelijkheden die de omgeving biedt. Alle bedrijven maken met name in hun beginfase intensief gebruik van lokale hulpbronnen (werknemers, adviseurs, kapitaalverschaffers, toeleveranciers, klanten e.d). Op de tweede plaats proberen de ondernemingen specifieke onderdelen van de omgeving ook te manipuleren of te beinvloeden in hun cigen voordeel. Als beïnvloeding niet mogelijk is kunnen ze proberen het bedrijf in te dekken of imimulun te maken voor 'gebreken' in de omgeving. Als ook dat niet mogelijk is trachten zij zich op meer of minder creatieve wijze aan te passen aan de externe omstandigheden. Terwijl het bij de eerstgenoemde categorie, i.e. het benutten van hulpbronnen, gaat om het profiteren van voordelen van de omgeving staat bij de overige drie categorieën centraal het tegenspel bieden tegen beperkingen in de omgeving. Hoewel we daarin niet moeten overdrijven blijkt uit het onderzoek toch dat waar de Randstadbedrijven profijt kunnen trekken van hun lokale omgeving, hun perifere tegenhangers meer of vaker worden geconfronteerd met (dreigende) knelpunten en belemmeringen, waaraan zij weerstand moeten bieden. Het punt is dat dit soms (niet altijd) aanzienlijke extra kosten met zich meebrengt. Neem bijvoorbeeld de stormachtige ontwikkeling van de computersector in de jaren tachtig. Waar automatiseringsbedrijven in de Randstad in die dagen kunnen profiteren van een sterke concentratie van hoog opgeleide informatici in de westelijke provincies, moeten collega's elders in het land vaak luid met de geldbuidel rammelen om geschikte arbeidskrachten van heinde en verre weg te lokken en/of moeten zij zwaar investeren in dure bedrijfsopleidingen. Hoe je het ook wendt of keert, de minder gunstig gevestigde bedrijven lijken in het nadeel te blijven ten opzichte van hun gunstig gesitueerde tegenhangers. Men kan zich nu eenmaal niet aan de eigen haren uit het moeras omhoog trekken, of...?

De tweede onderzoeksvraag die in het proefschrift wordt behandeld vraagt zich in elk geval of het zich teweer stellen van bedrijven tegen tekortkomingen van de externe omgeving wel echt zoden aan de dijk zet, met het oog op de genoemde kosten die daarmee gepaard kunnen gaan? Het antwoord daarop luidt als volgt. Je moet niet alleen kijken naar de kosten die gepaard kunnen gaan met het nemen van maatregelen tegen nadelen van de vestigingsplaats, maar ook naar wat zij opleveren. Het onderzoek levert aanwijzingen - geen bewijzen - dat de opbrengsten de kosten wel eens ruimschoots kunnen overstijgen. Neem bijvoorbeeld, een Leeuwardens bedrijf in het onderzoek, dat geen externe kapitaalverschaffer in de omgeving bereid vindt om flink in het bedrijf te investeren. Ten einde raad besluiten directie en 
personeel om het bedrijf zelf maar op te kopen. Liefst 23 werknemers blijken bereid om ieder voor minstens 50.000 gulden aandelen van hun eigen bedrijf te kopen. In de literatuur over werknemersparticipatie blijkt nu dat bedrijven met werknemersparticipatie beter presteren dan bedrijven zonder werknemersparticipatie, omdat in de eerstgenoemde groep ondernemingen de werkmotivatie onder het personeel veel hoger is dan in de laatstgenoemde. Een ander voorbeeld vinden we op het gebied van bedrijfsopleidingen. Perifere bedrijven die in vergelijking met hun centraal gevestigde collega's grotere investeringen moeten doen in aanvullende scholing van het personeel, omdat de kwaliteit van de externe arbeidsmarkt tekort schiet, zouden daar wel eens meer dan evenredig voordeel van kunnen hebben. Uit de economische literatuur blijkt namelijk dat bedrijven die de nadruk leggen op het intern opleiden van het personeel uiteindelijk beter functioneren dan bedrijven die vooral direct inzetbare mensen aannemen. Ook hier is de reden dat bij de eerste groep ondernemingen de motivatie en binding van het personeel met het bedrijf groter is dan bij de laatste groep. Een laatste voorbeeld betreft de afzetmarkt. In het onderzoek zien we dat waar de Randstadbedrijven minstens tien jaar na de oprichting hun omzet voor het overgrote deel nog steeds uit de afzetmarkt in het eigen vestigingsgebied halen, de perifere bedrijven al in een zeer vroeg stadium van hun ontwikkeling, zo niet meteen vanaf de start, hun produkten buiten het eigen vestigingsgebied en zelfs in het buitenland moeten afzetten om voldoende omzet te behalen. Zeker voor startende ondernemers is dat geen sinecure, maar indien zij daarin slagen levert het hun enorme leervoordelen op. Het management wordt immers al in een vroeg stadium gepokt en gemazeld in het overwinnen van een van de grootste barrières voor continuering van de groei, namelijk het binnendringen van ver verwijderde afzetmarkten. En naarmate een groeiend bedrijf dit tijdstip uitstelt wordt het probleem alleen maar groter. Dus samengevat kunnen we stellen dat het nemen van tegenmaatregelen tegen nadelen van de bedrijfsomgeving wel degelijk effectief kan zijn, omdat de opbrengsten of voordelen die daaruit kunnen voortvloeien de kosten ruimschoots kunnen overstijgen. Op deze manier kan er compensatie en zelfs overcompensatie ontstaan voor aanvankelijke vestigingsplaatsnadelen. In de economie duidt men situaties waarbij landen, regio's of individuele bedrijven nadelen omzetten in voordelen of een achterstand veranderen in een voorsprong aan met de term dymamische oncoenwichtigheid.

De derde onderzoeksvraag heeft betrekking op de uiteindelijke oorsprong van het groei en weerbaarheid van de onderzochte bedrijven. Met het antwoord op de tweede onderzoeksvraag dat weerstandsstrategieën tegen knelpunten in de omgeving dus wel degelijk effectief kunnen zijn, zijn we er immers nog niet. Er zijn soms grote investeringen mee gemoeid en ook hier geldt dat de kost voor de baat uitgaat. De middelen waarover een bedrijf beschikt om bepaalde investeringen op een bepaald moment te kunnen doen weerspiegelt de groei van dat bedrijf in de voorafgaande periode. De vraag is dus welke factoren of krachten ten grondslag liggen aan de eerste betekenisvolle 'groeistuip' van de onderzochte bedrijven. Deze bepaalt de armslag die het management heeft voor verdere ontwikkeling van de onderneming. $\mathrm{Bij}$ de Randstadbedrijven spelen, in overeenstemming met regionale onwikkelingstheorieën, de voordelen van het grootstedelijk vestigingsmilieu duidelijk een belangrijke rol. Het meest aansprekend is dat gebeurd bij een onderneming waarbij de lokale afzetmarkt zich zowat aan het bedrijf 'opdringt' met innovatieve ideeën. De onderne- 
ming pakt die ideeën op en verandert daardoor binnen enkele jaren van een klein eenmansbedrijf tot nationale marktleider in zijn branche. Bij de bedrijven in overig Nederland ligt de zaak echter anders. Zij illustreren niet zozeer de rol van de bedrijfsomgeving, maar veel meer de betekenis van de aard van de moederorganisatie waaruit ze zijn voortgekomen. Het blijkt dat deze bedrijven bepaalde sterke eigenschappen van het ouder-bedrijf hebben overgeërfd. Daardoor zijn zij van meet af aan uitstekend in staat om de beperkingen van een minder gunstige omgeving te overwinnen. De ondernemers die deze bedrijven hebben opgericht vervulden in hun vorige werkkring leidende posities in nationaal en internationaal opererende (niet noodzakelijk grote) ondernemingen. In deze sterk naar buiten gerichte moederorganisaties vergaren $\mathrm{zij}$ technische en commerciële kennis en vaardigheden en bouwen zij eigen contactennetwerken op die het regionale niveau verre overschr den. En het is precies deze buitenwaardse orientatie, die zij als het ware hebben overgeërfd van de organisaties waaruit zij zijn voortgekomen, die de snelgroeinde bedrijven buiten de Randstad zo kenmerkt. Daardoor hebben zij er bij wijze van spreken geen centje pijn van dat er in hun 'schrale' vestigingsregio nauwelijks of geen potentiële afnemers zijn gevestigd. Deze ondememers verkopen hun produkten al in een zeer vroeg stadium, de meesten meteen vanaf het begin, in alle uithoeken van het land en zelfs in het buitenland. Daarin verschillen zij sterk van het gemiddelde bedrijf in de periferie. De meeste van deze bedrijven zijn uitgesproken lokaal ge-oriënteerd terwijl ze dat, gezien de beperktere capaciteit van de omliggende afzetmarkt, juist het minst zouden moeten zijn. Het onderzoek suggereert dat hier een taak voor de overheid is weggelegd. Het is zaak dat het 'parochialisme' van het bedrijfsleven in perifere gebieden afneemt en hun actieradius zich uitbreidt. Dat lijkt niet alleen belangrijk voor de groei van de bestaande ondernemingen, maar meer nog voor de kwaliteit en de groeikansen van hun 'nageslacht', i.e. de nieuwe bedrijven die zij afsplitsen. 


\section{Curriculum vitae}

The author of the present thesis was born in 1955 in a small Dutch village, called Nijswiller (the rural district of Wittem). After he had completed secundary education and fulfilled his military service he went studying at the teacher training college of De Gelderse Leergangen in Nijmegen in the year 1976. In 1981 he qualified as a biology and geography teacher. In this same year he moved on from the teacher training college to the Catholic Universty of Nijmegen where he graduated in 1986 as an economic geograher. In the period 1986-1987 he fulfilled consultancy activities for the economic consultancy firm Buro Bartels and the conference agency Manager Meetings. In 1987 he joined the department of Economic Geography of the Nijmegen University, initially as an added researcher and between 1988 and 1993 as a PhD student. Between April 1993 and August 1993 he conducted research at the Small Business Research Centre of the Univesity of Cambridge (UK). The latter project deals with fast growing small and medium sized enterprises in unfavourable environments in the United Kingdom. Currently he is working out the results of this research. 
1 G MIK \& J H STIKKELBROEK, Verkuezingen in Rotterdam -- Amsterdam/Rotterdam 1985 Knag/EconomischGeografisch Instutuut Erasmus Universiteit Rotterdem 130 pp, 51 figs, 8 tabs ISBN 90-6809-009-7 Dfl 17,50

2 S MUSTERD, Verschillende structuren en ontwikkelingen van woongebieden in Tulburg -- Amsterdam 1985 Knag/Geografisch en Planologisch Instituut van de Vruje Universiteit Amsterdam 292 pp, 104 figs, 44 tabs ISBN 90-6809-010-0 Dfl 27,75

3 M J TITUS, Ubanisatie, integratie en demografische respons in Jakarta -- Amsterdam/Utrecht 1985 Knag/ Geografisch Instituut Rujksuniversiteit Utrecht 380 pp, 14 figs, 202 tabs ISBN 90-6809-012-7 Dil 39,50

4 H SCHENK, Views on Alleppey - Amsterdam 1986 Knag/nstituut voor Sociale Geografie Universiteit van Amsterdem 246 pp, 41 figs, 36 tabs ISBN 90-6809-011-9 Dn 29,50

5 P J BOELHOUWER \& F M DIELEMAN (red), Wonen in de stad - Amsterdam/Utrecht $1986 \mathrm{Knag} / \mathrm{Geogra-}$ fisch Instituut Ruksuniversiteit Utrecht 138 pp, 41 figs, 32 tabs ISBN 90-6809 0135 Dfl 19,50

6 P LUKKES \& J H M v ROODEN, De makelaardij in onroerende goederen in Nederland - Amsterdam/Groningen $1986 \mathrm{Knag} / \mathrm{Geografisch}$ Instituut Rijksuniversitet Groningen $102 \mathrm{pp}, 9$ figs, 28 tabs ISBN 90-6809-015-1 Out of print

7 P P P HUIGEN, Binnen of buten bereik? Een sociagl-geografiseh onderzoek in Zuidwest-Fneslend - Amsterdam/Utrecht $1986 \mathrm{Knag} / \mathrm{Geografisch} \mathrm{Instituut} \mathrm{Rujksuniversitest} \mathrm{Utrecht} 276$ pp, 58 figs, 72 tabs ISBN 90 6809-014-3 Dn 34,00

B V M v DALEN \& L v d LAAN (red), Werken aan de kust, verslag van het Knag symposium over de plannen tot uitbreidıng van de Nederlandse kust - Amsterdam 1986 Konınkluk Nederlands Aardnjkskundig Genootschap 78 pp, 8 figs, 2 tabs ISBN 90-6809-016-X Dfl 14,00

9 H KNIPPENBERG, Deeiname aan het lager onderwijs in Nederland gedurende de negentiende eeuw - Amsterdam $1986 \mathrm{Knag} /$ Instituut voor Sociale Geografie Universiteit van Amsterdam 268 pp, 29 figs, 81 tabs ISBN 90-6809-017-8 Dfl 29,00

$10 \mathrm{H} J$ A BERENDSEN (red), Het landschap van de Bommelerwaard - Amsterdam/Utrecht $1986 \mathrm{Knag} / \mathrm{Geogra}-$ fisch Instituut Rujksuniversiteit Utrecht 186 pp, 71 figs, 2 maps ISBN 90-6809-019-4 Df 34,50

$11 \mathrm{M}$ de SMIDT (red), Regionale statistiek organisatie en onderzoek - Amsterdam/Utrecht $1986 \mathrm{Knag} / \mathrm{Geogra}-$ fisch Instituut Rujksuniversiteit Utrecht 86 pp, 17 figs, 9 tabs ISBN 90-6809-020-8 Dfl 14,95

$12 \mathrm{~J}$ M $\vee$ MOURIK, Pollen profiles of slope deposits in the Galıcian area (NW Spain) - Amsterdam $1986 \mathrm{Knag} /$ Fysisch-Geografisch en Bodemkundig Laboratonum Universitet van Amsterdam 174 pP, 55 figs, 4 tabs ISBN 90-6809-018-6 Out of print

13 J J HARTS \& L HINGSTMAN, Verhuizingen op een nj - Amsterdam/Utrecht $1986 \mathrm{Knag} /$ Geogra fisch Instituut Rujksuniversitet Utrecht 312 pp, 54 figs, 108 tabs ISBN 90-6809-022-4 Df 38,50

14 A v SCHAJK, Colonsal control and peasant resources in Java - Amsterdam 1986 Knag/lnstituut voor Sociale Geografie Universiteit van Amsterdam 214 pp, 14 figs, 31 tabs ISBN 90-6809-021-6 Dfl 27,00

15 L L J M DIRRIX, T K GRIMMIUS \& P v d VEEN, The functioning of penodic markets in the Bombay Metropolitan Region - Amsterdam/Groningen $1986 \mathrm{Knag} / \mathrm{Geografisch}$ Instituut Rujksuniversiteit Groningen $200 \mathrm{pp}$, 38 figs, 47 tabs ISBN 90-6809-030-5 Out of print

16 J G BORCHERT, L S BOURNE \& R SINCLAIR (eds), Urban Systerns in Transition -- Amsterdam/Utrecht $1986 \mathrm{Knag} / \mathrm{Geografisch} \mathrm{Instituut} \mathrm{Rujksunverseit} \mathrm{Utrecht} 248$ pp, 41 figs, 48 tabs ISBN 90-6809 0283 Dn 24,90

17 P W BLAUW, Suburbanisatie en sociale contacten - Amsterdam/Rotterdam $1986 \mathrm{Knag} /$ Facultent der Economische Wetenschappen Erasmus Universiteit Rotterdam 168 pp, 68 tabs ISBN 90-6809 024-0 Dก 25,00

$18 \mathrm{H}$ J SCHOLTEN, R J $v$ de VELDE \& P PADDING, Doorstroming op de Nederlandse woningmarkt, geanalyseerd en gemodelleerd -- Amsterdam/Utrecht $1986 \mathrm{Knag} / \mathrm{Geografisch}$ Instituut Rıjksuniversiteit Utrecht $116 \mathrm{pp}$, 38 figs, 22 tabs ISBN 90-6809-025-9 DA 13,00

19 P M DIELEMAN, A W P JANSEN \& M de SMIDT (red), Metamorfose van de stad, recente tendenzen van wonen en werken in Nederlandse steden -- Amsterdam/Utrecht 1986 Knag/Geografisch Instituut Rijksuniversiteit Utrecht $134 \mathrm{pp}, 31$ figs, 22 tabs ISBN 90-6809-026-7 Out of print

20 E VOS, M NIEUWENHUIS, M HOOGENDOORN \& A SENDERS, Vele handen , vrouw en werk in Latıjns Amenka - Amsterdam $1986 \mathrm{Knag} / \mathrm{Geografisch}$ en Planologiseh Instituut van de Vruje Universiteit $210 \mathrm{pp}, 9$ figs, 7 tabs ISBN 90-6809-027-5 DN 30,00

$21 \mathrm{~J}$ H J DINTEREN \& H W ter HART (red), Geografie en kantoren 1985 verslag van een congres gehouden te Numegen op 29 november 1985 - Amsterdam/Nymegen 1986 Knag/Geogralisch en Planologisch Instituut Katholieke Universitert Numegen 144 pp, 15 figs, 15 tabs ISBN 90-6809-029-1 DIl 17,00

22 J VIJGEN, R ENGELSDORP GASTELAARS, Stedelıke bevolkungskategoneén in opkomst, stijlen en strategieén un het alledagge bestaan -- Amsterdam 1986 Knag/Instituut voor Sociale Geografie Universiteit van Amsterdam 122 pp, 3 figs, 40 tabs ISBN 90-6809-031-3 Dil 15,00

$23 \mathrm{H} \mathrm{J} \mathrm{MUCHER,} \mathrm{Aspects} \mathrm{of} \mathrm{loess} \mathrm{and} \mathrm{loess-denved} \mathrm{slope} \mathrm{deposits,} \mathrm{an} \mathrm{expenmental} \mathrm{and} \mathrm{micromorphological} \mathrm{ap-}$ proach - Amsterdam 1986 Knag/Fysisch-Geografisch en Bodemkundig Laboratonum Universiteit van Amsterdam 268 pp, 42 figs, 9 tabs ISBN 90-6809-032-1 Out of prant

24 P HENDRIKS, De relationele definitue van begnppen - Amsterdam/Nımegen $1986 \mathrm{Knag} /$ Geografisch en Planologisch Instrtuut Katholieke Universteit Numegen 282 pp, 28 figs, 7 tabs ISBN 90-6809-033-X Dfl 30,00

25 I M G KLEINPENNING (ed), Competition for rural and urban space in Latin Amenca, its consequences for low income groups - Amsterdam/N $/$ ymegen $1986 \mathrm{Knag} /$ Geografisch en Planologisch Instituut Katholieke Universiteit Nymegen 178 pp, 36 figs, 11 tabs ISBN 90-6809-034-8 Dfl 22,50

$26 \mathrm{~J}$ BUURSINK \& E WEVER (red), Regio en ontwhkkeling - Amsterdem/Numegen 1986 Knag/Geografisch-Planologisch Instituut Katholieke Universiteit Numegen 160 pp, 41 figs, 50 tabs ISBN 90-6809-035-6 Dil 20,00 
27 G CLARK, P DOSTAL \& F THISSEN (eds), Rural research and planning the Netherlands and Great Brtain Amsterdam $1987 \mathrm{Knag} /$ Instituut voor Sociale Geografie Universiteit van Amsterdam 88 pp, 6 figs, 4 tabs ISBN 90-6809-037-2 Dil 10,00

28 W M KARREMAN \& M de SMIDT (red), Redevoenngen en kleıne geschnften van Prof A C de Vooys - Amsterdam/Utrecht $1987 \mathrm{Knag} /$ Geografisch Instituut Rjksuniversitest Utrecht $156 \mathrm{pp}, 8$ figs, 5 tabs ISBN 90 6809-036-4 DI 21,70

29 G PEPERKAMP (red), Mens en milieu in de derde wereld - Amsterdam/Nymegen 1987 Knag/Geografisch en Planologisch Instituut Katholieke Universiteit Numegen 146 pp, 17 figs, 11 tabs ISBN 90-6809-038-0 Dfl 20,00

30 A R WOLTERS \& A PIERSMA, Beschermde reservaten? - Amsterdam/Groningen $1987 \mathrm{Knag} / \mathrm{Geografisch}$ Instituut Ruksuniversiteit Groningen 184 pp, 47 figs, 4 tabs ISBN 90-6809-039-9 Out of prunt

31 W J Y d BREMEN \& P H PELLENBARG (red), Het geografisch plechtanker eenheid in verscheidenheid Liber amiconm Rob Tamsma - Amsterdam/Groningen $1987 \mathrm{Knag} / \mathrm{Geografisch}$ Instutuut Rujksuniversiteit Groningen 336 pp, 58 figs, 22 tabs ISBN 90-6809-040-2 Dfl 35,00

32 G MIR, Segregatue in het grootstedelyjk mileu, theone en Rotterdamse werkelyjkheid -- Amsterdam/Rotterdam 1987 Knag/Economisch-Geografisch Instituut Erasmus Universiteit Rotterdam 252 pp, 48 figs, 45 tabs ISBN 90-6809-041-0 DI 25,00

33 H J M GOVERDE, Macht over de Markerruumte - Amsterdam/Nijmegen 1987 Knag/Geografisch en Planologisch Instituut Katholicke Universiteit Numegen 480 pp, 26 figs, 22 tabs ISBN 90-6809 042-9 DI 57,50

34 P P GROENEWEGEN, J P MACKENBACH \& M H STIJNENBOSCH (red), Geografie van gezondheid en gezondheidszorg - Amsterdam/Utrecht 1987 Knag/Geografisch Instituut Ruksuniversiteit Utrecht 132 pp, 25 figs, 19 tabs ISBN 90-6809-043-7 Dfl 19,70

35 R ter BRUGGE \& E WEVER (red), Energiebeleid, het Nederlandse energiebeleid in ruimteluk perspectief Amsterdam/Groningen/Numegen $1987 \mathrm{Knag} / \mathrm{Geografisch}$ Instituut Rujksuniversitet Groningen/Geografisch en Planologisch Instituut Katholeke Universiteit 132 pp, 21 figs, 18 tabs ISBN 90-6809-044-5 DN 18,00

36 J A v d SCHEE, Kujk op kaarten, een empinsch onderzoek naar het gebruk van geografische denkvaardigheden by het analyeeren van kaarten door leerlingen - Amsterdam 1987 Knag/Geografisch en Planologisch Instituut van de Vnje Universitejt Amsterdam 312 pp, 42 figs, 58 tabs ISBN 90-6809 045-3 DN 39,50

37 O VERKOREN \& J v WEESEP (eds), Spatial mobility and urban change - Amsterdam/Utrecht 1987 Knag/ Geografisch Instituut Ruksuniversiteit Utrecht 180 pp, 17 figs, 45 tabs ISBN 90-6809-051-8 DN 24,75

$38 \mathrm{M}$ W de JONG, New economic activities and regional dynamics - Amsterdam $1987 \mathrm{Knag} /$ Economisch-Geografisch Instituut Universiteit van Amsterdam 200 pp, 26 figs, 27 tabs ISBN 90-6809-046-1 Dn 29,00

39 A C M JANSEN, Bier in Nederland en Belgiè, een geografie van de smaak - Amsterdam 1987 Knag/Econo misch Geografisch Instituut Universiteit van Amsterdam 282 pp, 14 figs, 7 tabs ISBN 90-6809-047-X Dfl 37,50

40 Y C J BROUWERS, M C DEURLOO \& L de KLERK, Seleclieve verhusbewegingen en segregatie, de unvloed van de etnische samenstelling van de woonomgeving op verhusgedrag -- Amsterdam 1987 Knag/Instituut voor Sociale Geogra fie Universiteit van Amsterdam 112 pp, 9 figs, 22 tabs ISBN 90-6809-048-8 Dfl 16,00

41 R J SCHOUW \& F M DIELEMAN, Echtscheiding en woningmarkt - Amsterdam/Utrecht 1987 Knag/Geografisch Instituut Ruksuniversiteit Utrecht 98 pp, 8 figs, 21 labs ISBN 90-6809-049-6 Dil 14,95

42 J G GROENENDIJK, De positie van dorpen in het beleid van Nederlandse plattelandsgemeenten, een politiekgeografisch onderzoek naar de verdeling van woningbouwlokgties - Amsterdam $1987 \mathrm{Knag} / \mathrm{lnstituut}$ voor Sociale Geografie Universiteit van Amsterdam 314 pp 22 figs, 55 tabs ISBN 90-6809 050-X DII 31,50

43 J G BORCHERT \& J BUURSINK (red), Citymarketing en geografie - Amsterdam/Nijmegen 1987 Knag/Geografisch en Planologisch Instituut Katholieke Universiteit Nijmegen 172 pP, 32 figs, 14 tabs ISBN $90-6809-052-6$ Dfl 20,00

44 J J M ANGENENT \& A BONGENAAR (eds), Planning without a passport the future of European spatial plan ning - Amsterdam 1987 Knag/Stıchtıng Interuniversitair Instituut voor Soclaal-Wetenschappelijk Onderzoek 184 pp, 26 figs, 7 tabs ISBN 90-6809-053-4 Out of pnit

45 R C v d MARK, A H PERRELS \& J J REYNDERS, Kansen voor het Noorden, cen beleidsstrategisch onderzoek naar nieuwe technologie - Amsterdam/Utrecht $1987 \mathrm{Knag} /$ Geografisch Instituut Ruksuniversiteit Utrecht Economische Faculteit Vnje Universiteit Amsterdam 168 pp, 54 figs, 41 tabs ISBN 90-6809-054-2 Df 22,50

$46 \mathrm{~J} \mathrm{~J}$ STERKENBURG, Rural development and rural development policies cases from Afnia and Asıa -- Amsterdam/Utrecht $1987 \mathrm{Knag} / \mathrm{Geografisch}$ Instituut Rujksuniversiteit Utrecht $196 \mathrm{pp}, 13$ figs, 14 tabs ISBN 90 6809-055-0 Dก 26,50

47 C CORTIE, Alkmaar, van streekcentrum naar groeikem - Amsterdam 1987 Knag/Instituut voor Socıale Geografic Universiteit ven Amsterdam 204 pp, 28 figs, 39 Labs ISBN 90-6809-056-9 Dfl 25,00

48 J A A M KOK \& P H PELLENBARG (red), Buitenlandse bednjven in Nederland - Amsterdam/Groningen $1987 \mathrm{Knag} /$ Geografisch Instituut Rujksuniversitet Groningen $112 \mathrm{pp}, 17 \mathrm{figs}, 30$ tabs ISBN 90-6809-059-3 Out of print

49 T DIETZ, Pastoralists in Dire Straits, survival strategies and external interventions in a semi-and region -- Amsterdam $1987 \mathrm{Knag} /$ Instituut voor Sociale Geografie Universteit van Amsterdam 332 pp, 34 figs, 66 tabs ISBN 90-6809-057-7 DAl 43,00

$50 \mathrm{~F} J \mathrm{~J}$ H v HOORN, Onder anderen, effekten van de vestıging van Mediterranen un naoorlogse wujken - Amsterdam/Utrecht $1987 \mathrm{Knag} / \mathrm{Geografisch}$ Instituut Rujksuniversiteit Utrecht 226 pp, 36 figs, 55 tabs ISBN 90-6809-060-7 Dfl 29,70

51 M J DIJST \& C CORTIE, Universiteit en revitalısering - Amsterdam 1987 Knag/Instituut voor Sociale Geografie Universiteit van Amsterdam 140 pp, 6 figs, 13 tabs ISBN 90-6809-058-5 Dil 17,00

52 Planologie als kleurbepaling, de rol van toonasngevende instellingen en bednjven op de ontunkkelung van de Amsterdamse Museum- en Concertgebouwbuurt - Amsterdam $1987 \mathrm{Knag} / \mathrm{Centrum}$ Belendsadviserend Onderzoek 164 pP, 2 figs, 23 tabs ISBN 90-6809-061-5 Dfl 25,00

53 J VERHORST \& M H STIJNENBOSCH, Bednjvigheid en stadsvernieuwng, analyse van de bednjvigheidsontwhkkeling in enkele stadsvernieuwngsgebieden in Utrecht en Den Hagg - Amsterdam/Utrecht 1987 Knag/ Geografisch Instituut Ruksuniversiteit Utrecht 112 pp, 47 figs, 25 tabs ISBN 90-6809-063-1 DI 15,70 
54 B G J DRIESSEN, R VERHOEF \& J G P ter WELLE-HEETHUIS, Overheid en bevolkangsontwkkelungen, een onderzoek nasr autonome en niet-autonome bevolkongsontwikkelingen in Amhern en Utrecht Amsterdam/Utreeht $1987 \mathrm{Knag} / \mathrm{Geografisch}$ Instituut Rujksuniversiteit Utrecht 166 pp, 53 figs, 42 tabs ISBN 90-6809-064X Dfl 23,30

55 O A L C ATZEMA, P P P HUIGEN, A G A de VOCHT \& C R VOLKERS, De bereikbaarheid van voorzieningen in Noord Nederland - Amsterdam/Utrecht 1987 Knag/Geografisch Instituut Rujksuniversiteit Utrecht 220 pp, 49 figs, 122 tabs ISBN 90-6809-065-8 Dfl 24,00

56 P C BEUKENKAMP, G A HOEKVELD \& A MUDDE (red), Geografie en onderwistelevisie Amsterdam/Utrecht $1987 \mathrm{Knag} / \mathrm{Geografisch}$ Instituut Ruksuniversiteit Utrecht $222 \mathrm{pp}, 29$ figs, 6 tabs ISBN 90-6809-066-6 Dfl 26,50

57 G CARDOL, Ruimte voor agnbusuness-complexen, structuur, positie en dynamiek van het Noordlumburgse tuinbouwcomplex - Amsterdam/Numegen 1988 Knag/Geografisch en Planologisch Instituut Katholieke Universiteit Nijmegen 312 pp, 34 figs, 57 tabs ISBN 90-6809-067-4 Dfl 30,00

$58 \mathrm{M}$ JANSEN-VERBEKE, Leisure, recreation and tounsm in inner cities - Amsterdam/Numegen $1988 \mathrm{Knag} /$ Gcografisch en Planologiseh Instituut Katholieke Unıversiteit Nifnegen 316 pp, 61 figs, 51 tabs ISBN 90-6809-068-2 Out of print

59 A H H M KEMPERS-WARMERDAM, Vergnjzen in het groen, het bereik van ouderen en de bereikbaarheid van voorzıeningen in landelyke gebıeden - Amsterdam/Utrecht $1988 \mathrm{Knag} /$ Geografisch Instituut Ruksuniversiteit Utrecht 236 pp, 47 figs, 70 tabs ISBN 90-6809-069-0 Dfl 29,50

60 P J BOELHOUWER, De verkoop van woningwetwoningen - Amsterdam/Utrecht $1988 \mathrm{Knag} / \mathrm{Geografisch}$ Inst1tuut Rujksuniversitet Utrecht 208 pp, 49 figs, 122 tabs ISBN 90-6809-070-4 Df 29,30

61 A G J DIETVORST \& M C JANSEN-VERBEKE, De binnenstad kader van een sociaal perpetuum mobule, een literatuuratudie naar tıdsbesteding en binnenstadsgebruik - Amsterdam/Numegen $1988 \mathrm{Knag} / \mathrm{Geografisch}$ en Planologisch Instituut Katholeke Universiteit Nijmegen 240 pp, 10 tabs ISBN 90-6809-071-2 Dfl 30,00

62 H SCHRETTENBRUNNER \& J v WESTRHENEN, Empinsche Forschung und Computer im Geographie-unterncht, - Amsterdam $1988 \mathrm{Knag} /$ Centrum voor Educatıeve Geografie Vnje Universiteit $120 \mathrm{pp}, 27$ figs ISBN 90-6809-072-0 Out of print

$63 \mathrm{H}$ J A BERENDSEN \& H $\vee$ STEIJN (red), Nieuwe kartenngsmethoden in de fysische geografie -- Amsterdam/ Utrecht $1988 \mathrm{Knag} / \mathrm{Geografisch}$ Instituut Utrecht $176 \mathrm{pp}, 56$ figs, 24 tabs ISBN 90-6809 0739 Dfl 22,50

64 A G J DIETVORST \& J P M KWAAD (eds), Geographical research in the Netherlands 1978-1987 -- Amsterdam $1988 \mathrm{Knag} / \mathrm{IGU}$ Netherlands $262 \mathrm{pp}, 7$ figs, 2 tabs ISBN 90 6809-074 7 Dn 33,00

$65 \mathrm{~J} v$ WEESEP, Appartementsrechten, het gebruk van het splitsingregime - Amsterdam/Utrecht $1988 \mathrm{Knag} /$ Geografisch Instituut Rujksuniversiteit Utrecht 94 pp, 4 figs, 16 tabs ISBN 90-6809-075-5 Dfl 14,50

66 T W A EPPINK, Choice of mathematical models in geographic research considering alternatives - Amsterdam/ N 49 tabs ISBN 90-6809-076-3 Dn 30,00

67 J HINDERINK \& E SZULC-DABROWIECKA (eds), Successful nural development in Third World Countnes Amsterdam/Utrecht $1988 \mathrm{Knag} /$ Geografisch Instituut Rujksuniversitet Utrecht 256 pp, 14 figs, 20 tabs ISBN 90-6809-077-1 DA 31,50

68 S BARENDS, J D H HARTEN, J RENES, J VERHORST \& K E $\vee$ d WIELEN (red), Planning in het verleden - Amsterdam/Utrecht $1988 \mathrm{Knag} / \mathrm{Geografisch}$ Instituut Rıjksunıversitet Utrecht 192 pp, 71 figs ISBN 906809-078-X Dfl 26,00

69 J MANSVELT BECK, The nse of a subsidized penphery in Spain -- Amsterdam 1988 Knag/Instituut voor Sociale Geografie Universiteit van Amsterdam 286 pp, 15 figs, 28 tabs ISBN 9068090798 DIl 37,50

70 S SMITH, Kleinschalige industne in Latıjns Amenka, een studie van de ontwikkelingsmogelıkheden in AguascaLentes, Mexıco - Amsterdam/Nımegen $1988 \mathrm{Knag} /$ Geografisch en Planologisch Instituut Katholieke Unıver siteit Numegen 422 pp, 4 figs, 16 tabs ISBN 90-6809 080-1 Dn 42,50

71 W DWARKASING, D HANEMAAYER, M de SMIDT \& P P TORDOIR, Rumte voor hoogwaardige kantoren, onderzock naar toplocatıes voor de commerciēle kantorensestor - Amsterdam/Utrecht/Leiden/Delft $1988 \mathrm{Knag} /$ Geografisch Instituut Ryjksuniversiteit Utrecht/Research voor Bcleıd/Inro-tno 112 pp, 7 figs, 44 tabs ISBN 90 6809-081-X Out of print

72 P J KORTEWEG, Dynamiek en ummobiliteit in naoorlogse woonwijken in Alkmaar, Haarlem en Purmerend Amsterdam/Utrecht $1988 \mathrm{Knag} / \mathrm{Geografisch}$ Instıtuut Rujksuniversiteit Utrecht 144 pp, 21 ligs, 34 tabs ISBN 90-6809-082-8 Df 20,90

73 P J WUERS, Land prices in Tokyo - Ansterdam $1988 \mathrm{Knag} /$ Economisch-Geografisch Instituut Universiteit van Amsterdam 84 pp, 12 figs, 8 tabs ISBN 90-6809-084-4 Dfl 47,50

$74 \mathrm{~J}$ v MOURIK (red), Landschap in beweging, ontwikkeling en bewoning van een stu1fzandgebied in de Kempen -Amsterdam $1988 \mathrm{Knag} /$ Faculteit Ruimtelyke Wetenschappen Universiteit van Amsterdam 197 pp, 95 figs, 1 tab ISBN 90-6809-083-6 Dfl 30,00

75 W J M OSTENDORF, Het social profiel van de gemeente, woonmuleudifferentiatie en de vorming van het stadsgewest Amsterdam -- Amsterdam $1988 \mathrm{Knag} /$ Instituut voor Sociale Geografie Universiteit van Amsterdam 192 pp, 12 figs, 26 tabs ISBN 90-6809-085-2 Dil 23,00

$76 \mathrm{~J}$ de BRUIN \& J A KOETSIER (red), De kracht van de regio, sociaal-economische ontwikkelingsmogelıjkheden van de regio - Amsterdam $1988 \mathrm{Knag} /$ Instıtuut voor Sociale Geografie Unuversitet van Amsterdam 104 PP, 12 figs, 6 tabs ISBN 90-6809-086-0 Dn 15,00

77 A G M v d SMAGT \& P H J HENDRIKS (red), Methoden op een keerpunt, opstellen aangeboden aan prof drs $P$ J W Kouwe - Amsterdam/Niımegen $1988 \mathrm{Knag} /$ Geogra fisch en Planologisch Instituut Katholieke Universiteit Numegen 170 pp, 29 figs, 10 tabs ISBN 90-6809-087-9 Dfl 25,00

$78 \mathrm{C}$ v d POST, Migrants and migrant-labour absorption in large and small centres in Swazuland - Amsterdam/Utrecht $1988 \mathrm{Knag} /$ Geografisch Instituut Rujsuniversitelt Utrecht $310 \mathrm{pp}, 32$ figs, 84 tabs ISBN 90 6809-088-7 Df 35,00 
79 L J de HAAN, Overheid en regronale untegretue van de savanne in Togo 1885-1985 - Amsterdam 1988 Knag' Instutuut voor Socuale Geografie Univerartett van Amaterdam 304 pp, 31 figs, 65 tabs ISBN 90-6809-089-5 Df 33,00

80 L H v WINGAARDEN-BAKKeR \& J J M v d MEER (eds), Spatual sciences, research un progress Proceodings of the symposium "Spatial sciences, rescarch in progress" - Amsterdam 1988 Knag/ Faculteit Ruimtelijke Wetenschappen Universiteit van Ansterdem 112 pp, 16 figa, 2 tabs ISBN 90-6809-091-7 Dn 24,00

81 F M H M DRIESSEN \& J H v HOUWELINGEN, Vnje tud en korte verblyfsrecreatie - Amsterdam/Utrecht $1988 \mathrm{Knag} /$ Bureau Dnessen 256 pp, 25 figa, 146 Labs ISBN 90-6809-095 X Df 15,00

82 G HOEKVELD-MEIJER \& G J SCHUTTE, Aardnjkskunde gebiedenderwjs, tekst en uttleg buj het schnjven, lezen, denken en leren over gebieden en verschunselen un gebieden - Amsterdam 1988 Knag/Centrum voor Educatieve Geografie Vnje Universiteit Amsterdam 252 pp, 76 ligs, 12 tabs Out of print

83 P K DOORN, Sociel atructure and spatial mobuty composition and dynamics of the Dutch labour force Amsterdam/Utrecht $1989 \mathrm{Krag} / \mathrm{Geografisch}$ Instituut Ruksuniversitett Utrecht $262 \mathrm{pp}, 72$ figs, 41 labs $15 B N$ 90-6809-092-5 DI 31,50

B4 A LOEVE, Buitenlandse ondernemingen in regionaal perspectıef, vestıgungsstrategieèn en regionale effecten van buitenlandae bednjven in Nederland - Ansterdam/Utrecht 1989 Knag/Geografisch Instjtuut Rujksuniverstent Utrecht 272 pp, 49 figs, 78 tabs ISBN 90-6809-093-3 Dfl 32,00

85 D H de BAKKER, Ruraal nederzettingenpatroon en belend, ontwikkeingen in Zundwest-Fresland - Amsterdam/Utrecht 1989 Knag/Geografiseh Instutut Rujksuniversitest Utrecht 230 pp, 32 figs, 68 tabs ISBN 90 6809-094-1 Dil 29,00

86 L J PAUL (ed). Post-war development of regional geography, with special attention to the United Kingdom, Belgium, and the Netherlands -- Amsterdam/Utrecht $1989 \mathrm{Knag} / \mathrm{Geografisch}$ Instituut Ruksuniversiteit Utrecht 88 pp, 15 figs, 5 tabs ISBN 90-6809-096-8 DII 14,00

87 P HOEKSTRA, Ruver outhow, depositional processes and cosstal morphodynamics in a monsoon-dominated deltaic environment, East Java, Indonesia - Amsterdam/Utrecht 1989 Knag/Geografisch Instituut Rujksuntversiteit Utrecht 220 pp, 77 figs, 24 tabs ISBN 90-6809-097-6 Df 28,50

88 E LENSINK, Intermediaure diensten un landelıjke gebieden -- Amsterdam/Nujmegen 1989 Knag/Faculteit Beleidswetenschappen Katholieke Universitet Nijmegen 246 pp, 21 figs, 65 tabs ISBN 90-6809-098-4 Dfl 30,00

89 P P P HUIGEN \& M C H M $\vee$ d VELDEN (red), De achterkant van verstedelukt Nederland, de positie en funktic van landeluke gebieden in de Nederlandse samenleving - Amsterdam/Utrecht $1989 \mathrm{Krag} /$ Geografiseh Instituut Rujksuniversiteit Utrecht 181 pp, 25 figs, 46 tabs ISBN 90-6809-100-X Out of pnnt

$90 \mathrm{~J} \mathrm{H} \mathrm{I}$ v DINTEREN, Zakeluke diensten en middelgrote steden, een onderzoek nasr dienstverlentngsbednjven in Noord-Brabant, Gelderland en Overjısel - Amsterdam/Numegen 1989 Knag/Faeulteit der Beleıdswetenschappen Katholieke Universiteit Numegen 312 pp, 28 figs, 84 labs ISBN 90-6809-099-2 Df 40,00

91 L V LAAN, H SCHOLTEN \& G A v d KNAAP, Het regional abeidsannbod in Nederland - Amsterdam/ Rotterdam 1989 Knag/Economisch-Geografisch Instituut Erasmus Universitett Rotterdam 128 pp. 27 figs, 28 tabs ISBN 90-6809-101-8 DIl 17,50

92 C CLARK, P HUIGEN \& F THISSEN (eds), Planning and the future of the countryside Great Bntan and the Netherlands - Amsterdam 1989 Knag/nnstituut voor Socıle Geografie Universiteit van Amsterdam 240 pp, 25 figs, 43 tabs ISBN 90-6809-102-6 Dil 35,00

93 J A $~$ DEN BERG. Venabuty of parameters for modelling sol motsture conditions, studies on loamy to sulty souls on marly bedrock in the Ardéche drainage basun, France - Amsterdam/Utrecht $1989 \mathrm{Knag} / \mathrm{Geogra}$ lisch Instituut Ruksuniversitest Utrecht 214 pp, 76 figs, 16 tabs ISBN 90-6809-103-4 Df 28,50

940 VERKOREN, Huzen op de hoogvlakte, een residentieel geografische verkenning van La Paz, Bolivia -- Amsterdam/Utrecht $1989 \mathrm{Knag} /$ Geografisch Instituut Ruksuniversiteit Utrecht 210 pp, 29 figs, 16 tabs ISBN 90 6809-104-2 Dfl 32,00

95 G MIK (red), Herstructurenng in Rotterdem, modermisenng en intemationalisenng en de Kop van Zuid - Amsterdam/Rotterdam 1989 Knag/Economisch Geografisch Instituut Erasmus Universiteit Rotterdam 324 pP 86 figs, 54 tabs ISBN 90-6809-105-0 Dก 30,00

96 P BEEKMAN, P v LINDERT, J POST \& W PRINS, Huisvestingsbeleid en informele bouw in de derde wereld - Amsterdam $1989 \mathrm{Knag} /$ Instituut voor Sociale Geografie Universiteit van Amsterdam 174 pp, 9 figs, 25 tabs ISBN 90-6809-106-9 DI 30,00

97 J G L PALTE, Upland farming on Java, Indonesia - Amsterdam/Utrecht $1989 \mathrm{Knag} / \mathrm{Geografiach}$ Instituut Rujksuniveriteit Utrecht 256 pp, 15 figs, 38 tabs ISBN 90-6809-107-7 Dfl 34,50

98 P v GENUCHTEN, Movement mechanisms and slide velocity vanations of landslides in varved clays in the French Alps - Amsterdam/Utrecht $1989 \mathrm{Knag} /$ Geografisch Instituut Rujksuniversiteit Utrecht 160 pp, 70 figs, 17 tabs ISBN 90-6809 108-5 DI 25,00

$99 \mathrm{M}$ de SMIDT \& E WEVER (eds), Regional and local economic policies and technology - Amsterdam/ Utrecht/Nijmegen $1989 \mathrm{Kag} /$ Geografisch Instituut Rjksuniversiteit Utrecht/Geografisch en Planologisch Inst1tuut Katholieke Universiteit Nijmegen 156 pp, 53 figs, 36 tabo ISBN 90-6809-109 3 Dfl 24,00

100 P J H RIEMENS, On the foreign operations of third world firms - Amsterdam 1989 Knag/Instituut voor Sociale Geografie Universiteit van Amsterdam 148 pp, 20 tabs ISBN 90-6809 110-7 Df 30,00

101 G B M PEDROLI, The nature of landscape, a contrbution to landscape ecology and ecohydrology with examples from the Strjper As landocape - Amsterdam 1989 Knag/Fysisch-Geografisch en Bademkundig Laboratonum Universiteil van Amsterdam 164 pp, 43 figs, 18 tabs ISBN 90-6809-111-5 DII 25,00

102 H LEENAERS, The dispersal of melal mining wastes in the catchment of the nver Geul, Belguum-the Netherlands - Amaterdam/Utrecht $1989 \mathrm{Knag} / \mathrm{Geografisch}$ Instituut Rujksuniveraitent Utrecht 230 Pp, 95 figs, 52 labe ISBN 90-6809-112-3 DI 30,00

103 G A HOEKVELD, G SCHOENMAKER \& J v WESTRHENEN, Wijkende grenzen - Amsterdam 1989 Krag/ c..- - - D. 
105 W BLEUTEN, De verwatenng van meststoffen, analyse en modellenng van de relatiea tussen landgebruuk en wateriwaliteit in het stroomgebied van de Lengbrocker Wetenng - Amsterdam/Utrecht $1990 \mathrm{Knag} / \mathrm{Geogra}-$ fisch Instituut Rulssuniveraiteit Utrecht 262 pp, 80 figs, 31 Lebs ISBN 90-6809-115-8 Df 37,00

$106 \mathrm{~J} \vee$ WEESEP \& P KORCELLI (ods), Residential mobulty and social change, studies from Poland and the Netherlands - Amsterdam/Utrecht $1990 \mathrm{Knag} / \mathrm{Geognafisch}$ Instituut Rukksunivenitedt Utrecht $182 \mathrm{pp} 46$ figs, 34 tubs. ISBN 90-6809-116-6 Dก 29,50

107 M v HERWINEN, R JANSSEN \& P RJTVELD, Herbestemming van landbouwgrond - Amsterdam 1990 Knag/Instutuut Mulieuvraagstukken Vnje Universitell 110 pp, 33 figs, 12 tabs ISBN 90-6809-117-4 D/1 25,00

108 D H DRENTH, De unformatica-sector in Nederland tussen njp en groen - Amstendam/Numegen 1990 Knag/ Paculteit der Beleidswetenschappen Katholeke Universitet Njjmegen 268 pp, 24 figs. 87 labs ISBN 90-6809118-2 DA 37,50

109 H KNOL \& MANSHANDEN, Functionele samenhang in de noordvleugel van de Randstad - Amsterdam/ Utrecht $1990 \mathrm{Kgag} /$ Economisch-Geografiseh Instituut Universitert van Amsterdam/Geografisch Instıtuut Rujksunversitelt Utrecht 112 pp, 26 figs, 27 tabs ISBN 90-6809-119-0 Dfl 19,50

110 C D EYSBERG, The Califomian wine economy - Amsterdam/Utrecht $1990 \mathrm{Knag} / \mathrm{Geografisch}$ Instuluut Rulksuniversiteit Utrecht 272 pp, 64 figs, 26 tabs ISBN 90-6809 121-2 DII 29,75

111 J W A DUKMANS, Aspects of geomorphology and thermoluminescence dating of cold climate colsan sands Amsterdam/Utrecht $1990 \mathrm{Krag} / \mathrm{Geografisch}$ Instituut Ruksuniversitett Utrecht 256 pp, 119 figs, 19 tabs ISBN 90-6809-120-4 Dfl 36,50

$112 \mathrm{H}$ ter HEIDE (ed), Technological change and spatial policy - Amsterdam/Utrecht $1990 \mathrm{Knag} /$ Geografisch Instutuut Ruksuniversitet Utrecht 218 pp, 9 figs, 31 tabs ISBN 90-6809-122-0 DIl 29,00

113 I L M v HEES, De ontwzkkeling van een woningmarktmodel en zujn toepassing op Italie Arnsterdam/ $\mathrm{N}_{1} / \mathrm{me}$ gen $1990 \mathrm{Knag} /$ Faculteit der Beleidgwetenschappen Katholueke Universiteit Nımegen 196 pp, 12 figs, 26 tabs ISBN 90-6809-123-9 Dก 35,00

114 M R HENDRIKS, Regionalisation of hydrological data effects of lithology and land use on storm runoff in cast Luxembourg - Amsterdam/Utrecht $1990 \mathrm{Knag} /$ Geografisch Instituut Ruksuniversitet Utrecht 174 pp, 23 figs, 50 labs ISBN 90-6809-1247 DI 26,00

115 P H RENOOY, The unformal economy - Amsterdam 1990 Knag/Regioplan 204 PP, 12 figs, 21 tabs ISBN 90-6809-125-5 Dfl 35,00

$116 \mathrm{~J}$ H T KRAMER, Luchthavens en hun uitstraling - Amsterdam/Numegen $1990 \mathrm{Knag} /$ Faculteit der Beleidswetenschappen Katholeke Universiteit Nijmegen 312 pp, 47 figs, 60 tabs ISBN 90-6809 126-3 Df 55,00

$117 \mathrm{M}$ de KWAASTENIET, Denomination and prumary education in the Netherlands 1870-1984 - Amsterdam/ Florence $1990 \mathrm{Knag} /$ Instutuut voor Sociale Geografie Universiteit van Amsterdam/European University Insti tute Florence 268 pp, 28 figs, 39 tabs ISBN 906809 127-1 D 36,00

118 W P M F IVENS, Almosphenc deposition onto forests an analysis of the deposition vanabulty by means of throughfall measurements - Amsterdam/Utrecht $1990 \mathrm{Knag} / \mathrm{Geografisch}$ Instituut Rujksuniversiteit Utrecht $156 \mathrm{pp}, 53$ figs, 36 tabs ISBN 90-6809-128-X Dก 25,00

119 R HASSINK, Herstructurenng en unnovaliebevordenng in hat Ruhrgebied -- Amsterdam/Utrecht 1990 Knag/Geografisch Instituut Rujksuniversiteit Utrecht 122 pp, 20 figs, 18 tabs ISBN 90-6809 120-8 Df 24,00

120 P P SCHOT,, Solute transport by groundwater flow to weiland ecosystems, the environmental impact of human activities - Amsterdam/Utrecht 1991 Knag/Geogrefisch Instituut Ryksuniversitet Utrecht 136 pp, 27 figs, 9 labs ISBN 90-6809-130-1 Df 25,00

121 S Den HENGST \& B de PATER (red), Externe relaties en regionale ontwikkeling voorbeelden uit Spanje en Portugal - Amsterdam/Utrecht 1991 Knag/Geografisch Instituut Rujksuniversiteit Utrecht 198 pp, 31 ligs, 27 tabs ISBN $90-6809131-X$ Dn 29,50

$122 \mathrm{~J}$ KROES, Onvolledige opstrek op de Nederlandse zandgronden - Amsterdam/Utrecht $1991 \mathrm{Knag} / \mathrm{Geografisch}$ Instituut Ruksuniversiteit Utrecht 256 pp, 65 figs ISBN 90-6809-132-8 Dfl 35,00

123 H S VERDUIN-MULLER, Serving the knowledge-based society research on knowledge products -- Amster$\mathrm{dam} /$ Utrecht $1991 \mathrm{Knag} /$ Geografisch Inst Universitet Utrecht 116 pp, 3 figs ISBN 90-6809 133-6 DII 24,00

124 F MULDER, Assessment of landslude hazard - Amsterdam/Utrecht 1991 Knag/Geografisch Instituut Rujksuniversiteit Utrecht 156 pp, 59 figs, 25 tabs ISBN 90-6809 134-4 Df 29.50

125 M VIS, Processes and pattems of erosion in natural and disturbed Andean forest ecosystems - Amsterdam 1991 Knag/Fysisch Geografisch en Bodemkundig Laboratonum Universiteit van Amsterdam 190 pp, 70 figs, 40 tabs ISBN $90-6809-136-0$ Df 12,00

126 V EIFF, Beleid voor bednjfsterreinen - Amsterdam 1991 Knag/Instituut voor Sociale Geografie Universiteit van Amsterdam 214 pp, 13 figs, 18 tabs ISBN 90-6809-135-2 Dfl 37,50

127 O ATZEMA, Stad uit, stad in, residentuele suburbanisatie in Nederland - Amsterdam/Utrecht $1991 \mathrm{Knag} /$ Geografisch Instutuut Rujksuniversitest Utrecht 274 pp, 57 figs, 80 tabs ISBN 90-6809-137-9 Dfl 37,50

128 M HULSHOF, Zatopec moves, networks and remittances of US-bound migrants from Oaxaca, Mexico - Amsterdam 1991 Knag/Instituut voor Sociale Geografie Universiteit van Amsterdam 106 pp, 6 figs, 14 tabs ISBN 90-6809-138-7 Dfl 18,50

129 J M J DOOMERNIK, Turkse moskeeēn en mantschappelijke partucipatıe, de instıtutionaluserung van de Turkse Islam in Nederland en de Duitse Bondsrepubliek - Amsterdam 1991 Knag/Instituut voor Sociale Geografie Unversilett van Amsterdam 200 pp, 30 figs, 1 tab ISBN 90-6809-139-5 Di 32,50

$130 \mathrm{M}$ de SMIDT, A GRANBERG \& E WEVER (eds), Regional development strategies and temtonal production complexes, Dutch-USSR perspective - Amsterdam 1991 Knag 216 pP, 25 figs, 40 tabs ISBN 90-6809 $140-9$ DI 29,50

131 P MISDORP, Centrale begnppen un de sociale geografie, cen conceptuele analyse van engelstaluge leerboeken Amsterdarn 1991 Iparto/Knag 316 pp, 39 ligs, 51 tabs ISBN 90-6809-141-7 Df 39,50

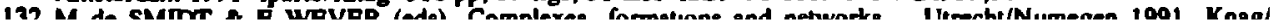


133 I I Y CASTEL, Late Holocene colan dnft sands in Drenthe - Amsterdam/Utrecht 1991 Krag/Geografisch Instituut Ruksouniversiteit Utrecht 162 pp, 49 figs, 15 tabs ISBN 90-6809-143-3 Dil 29,50

134 J G BORCHERT \& M de KRUYF, Bevolkungsgroei ter wille van het voorzienungennivesu' - Utrecht 1991 Knag/Faculteit Ruuntelyke Wetenschappen Universiteit Utrecht 78 pp, 4 figs, 15 tabs ISBN 90-6809-144-1 Dfl 18,00

$135 R$ v d VAART, Educatief ontwerpen ma geografie, cen studie bareffende de structurenng van geografische kennis voor educatieve doeleinden - Utrocht 1991 Knag/Facultent Ruimteljke Wetenschappen Rujksuni versitest Utrecht 256 pp, 84 figs ISBN 90-6809-145-X Dก 36,00

136 P y LINDERT, Huisvestıgingsstrategieèn van lage-inkomensgroepen in Lo Paz - Utrecht 1991 Kneg/ Faculteit Ruimtelyke Wetenschappen Rujksuniversiteit Utrecht 320 pp. 34 figs, 26 tabs ISBN 90-6809-146-8 Df 34, SO

137 J M M v AMERSFOORT \& H KNIPPENBERG (eds), States and nations the reburth of the 'nationalities question' in Europe - UtrechUAmsterdam 1991 Knag/Instiluut voor Sociale Geogrefie Universiteit van Amsterdam 198 pp, 20 figs, 12 tabs ISBN 90-6809-147-6 D 29,50

138 P v TEEFFELEN, Dienstencentra en rurale ontwikkeling, een onderzoek naar het aanbod en gebrulk van overheidsdiensten un MaL, Afrka - Utrecht 1992 Krag/Facultelt Ruimtelıke Wetenschappen Rujksuniversiteit Utrecht 256 pp, 70 figs, 40 tabs ISBN 90-6809-148-4 DI 34,50

139 T H M V d LOOP, Industnal dynamics and fragmented labour markets Construction firms and labourers in India - UtrechU/Amsterdam 1992 Knag/Instituut voor Sociale Geografie Universitest van Amsterdam 350 pp. 30 figs, 71 tabs ISBN 90-6809-149-2 Dil 49,50

$140 \mathrm{H} \vee \mathrm{d}$ WUSTEN (Ed), The urban political arena, geographies of public admunistration - Utrecht/Amsterdam 1992 Knag/Instituut voor Sociale Geogralie Universiteit van Amsterdam 192 pp, 28 figs, 12 tabs ISBN 90 6809-150 6 DI 35,00

I41 B D HOEKSTRA, Informatıenetwerken rondom bednjven, de bibliotheek als informatieleverancier voor het bednjfsleven - Utrecht/Groningen $1992 \mathrm{Knag} /$ Faculteit Ruumteljke Wetenschappen Rujksuniversiteit Groningen 112 pp, 16 figs, 8 tabs ISBN 90-6809-151-4 Dfl 25,00

$142 \mathrm{H}$ SCHRETTENBRUNNER \& J v WESTRHENEN (eds), Empincal research and geography teaching -. Utrecht/Amsterdam 1992 Knag/Centrum voor Educatieve Geografie Vnje Universitett Amsterdam 190 pp, 48 figs, 38 tabs ISBN $90-6809-152-2$ Dfl 30,00

$143 \mathrm{~J}$ v BECKUM \& C v d BURG, Naar een onlune videotex geografisch informatiesysteem voor educatieve toepassingen, het Giset Project 1987-1992 - Utrecht 1992 Knag/Faculteit Ruimtelıjke Wetenschappen Ruksuniversiteit Utrecht 224 pp, 19 figs, 30 tsbs ISBN 90-6809-153-0 Df 29,50

144 C P TERLOUW, the regional geography of the workd system extemal arens, penphery, semipenphery, core - Utrecht 1992 Knag/Faculteit Ruinteluke Wetenschappen Rujksuniversiteit Utrecht 240 pp. 74 figs, 39 tabs ISBN 90-6809 156-5 Dil 34,50

145 R HASSINK, Regional innovation policy case-studies from the Ruhr Area, Baden-Wurttemberg and the North East of England - Utrecht $1992 \mathrm{Knag} /$ Facultett Ruimtelujke Wetenschappen Rujksuniversitet Utrecht 196 pp, 27 figs, 28 labs ISBN 90-6809-155-7 Dil 29.00

I46 H REITSMA. T DIETZ \& L de HAAN (eds). Coping wnth semiandity, how the rural poor survive in dry season environments - UtrechU/Amsterdam 1992 Knag/Instituut voor Sociale Geografie Universiteit van Amsterdam 202 pp, 18 figs, 19 tabs ISBN 90-6809-154-9 Dfl 32,00

147 M HESSELS, Locational dynamics of business services, an untrametropolitan study on the Randstad Holland Utrecht 1992 Knag/Faculteit Rumteluje Wetenschappen Rujksuniversitest Utrechl 232 pp, 25 figs, 79 tabs ISBN 90-6809-157-3 DN 34,50

148 J KANT, Geografen en planologen op de arbeidsmarkt, het sueces op de abeidsmarkt van geografen en plano logen, afgestudeerd in de penode september 1987 - augustus 1990 - Utrecht $1992 \mathrm{Krag} / \mathrm{Stuchtung}$ Geografen werk 176 pp, 39 figs, 41 labs ISBN 90-6809 $15 B$ I Dn 26,00

149 R v d VAART (red), Aardnjkskunde in de basisvorming, terugbluk en perspectieven - Utrecht $1992 \mathrm{Knag}$ Faculteit Rumieljke Wetenschappen Rujksuniversitest Utrecht 168 PP ISBN 90-6809-159-X Dn 32,50

150 M R SCHEFFER, Trading places, fashion, retalers and the changing geography of clothing production Utrecht 1992 Knag/Faculteit Ruuntelyke Wetenschappen Rujksuniversiteit Utrecht 270 pp, 37 figs, 34 tabs ISBN 90-6809-160-3 DI 29,50

151 P P GROENEWEGEN \& P P P HUIGEN (eds), Micro-macro vrasgstukken in de sociologie en de sociale geo grafie - Utrecht $1992 \mathrm{Kng} /$ Facultet Rumtelyke Wetenschappen Ryjksuniversitet Utrecht 140 pp, 15 figs, 9 tabs ISBN 90-6809-161-1 Din 25,00

152 P v TEEFFELEN, L v GRUNSVEN \& O VERKOREN (eds), Possibilities and constraints of GIS applications in developing countnes, Proceedings of the Gisdeco seminar organized by the GIS Working Group of the Facul ty of Geographical Sciences, Utrecht University, 7-8 Apnl 1991 - Utrecht 1992 Krag/Faculteit Ruimtelujke Wetenschappen Ruksuniversiteit Utrecht 126 pp, 16 figs, 11 labs ISBN 90-6809-162-X D fl 24,50

153 L J PAUL, P P P HUIGEN \& C R VOLKERS (eds), The changing function and position of rural areus in Europe -- Utrecht 1992 Knag/Facultett Ruimtelyjke Wetenschappen Rujksuniversiteit Utrecht ca 200 pp ISBN 90-6809-163-8 Dfl 30,00

154 P LUCAS \& G M R A v OORT, Dynamiek in een stadsrandzone, werken en wonen in de stadsrandzone van de agglomeratie Utrecht - Utrecht $1993 \mathrm{Knag} /$ Faculteit Ruimteluke Wetenschappen Universiteit Utrecht 396 pp, 105 figs, 41 tabs, 20 photographs ISBN 90-6809-164-6 Dी 48,00

155 E DIRVEN, J GROENEWEGEN \& $S$ v HOOF (eds). Stuck in the region? Changing scales for regtonal idenity - Utrecht 1993 Knag/Vereniging van Utrechlse Gcografie Studenten Vugs 126 pP, 17 figs, 2 labs ISBN 90 6809-165-4 Dfl 27,50

156 G DRAAJJERS. The vanabulty of atmosphene deposition to forests, the effects of canopy structure and loresi edges - Utrecht 1993 Knag/Facultent Ruuntelyke Wetenachappen Universitent Utrecht ca 208 pP ISBN 90 
157 A P J de ROO, Modelling surface runoff and soil erosion in catchments using Geographical Information Systems; Validity and applicability of the 'answers' model in two catchments in the loess area of South-Limburg (The Netherlands) and one in Devon (UK) - Utrecht 1993: Knag/Faculteit Ruimtelijke Wetenschappen Universiteit Utrecht. 295 pp, 84 figs, 85 tabs. ISBN 90-6809-167-0 Dfl 36,00

158 R VERHOEFF, De weg naar de podia; ruimtelijke aspecten van het bezoek aan podiumkunsten in Nederland Utrecht 1993: Knag/Faculteit Ruimtelijke Wetenschappen Universiteit Utrecht. ca 208 pp. ISBN 90-6809-168-9 Dfl 35,00

159 H ZONDAG, Regio en bedrijfseconomische vitaliteit - Utrecht 1993: Knag/Faculteit Ruimtelijke Wetensehappen Universiteit Utrecht. 208 pp. ISBN 90-6809-169-7 Dfl 34,50

160 T SPIT, Strangled in structures; an institutional analysis of innovative policy by Dutch municipalities - Utrecht 1993: Knag/Paculteit Ruimtelijke Wetenschappen Universiteit Utrecht. 192 pp, 36 figs, 11 tabs. ISBN 90-6809170-0 Dfl 32,50

161 J HAUER \& G HOEKVELD (eds), Moving regions - Utrecht 1993: Knag/Faculteit Ruimtelijke Wetenschappen Universiteit Utrecht. 280 pp, 74 figs, 11 tabs. ISBN 90-6809-173-5 Df 34,50

162 A BARENDREGT, Hydro-ecology of the Dutch polder landscape - Utrecht 1993: Knag/Faculteit Ruimtelijke Wetenschappen Universiteit Utrecht. ca 208 pp. ISBN 90-6809-175-1 Dfl 34,50

163 G B M HEUVELINK, Error propagation in quantitative spatial modelling applications in Geographical Information Systems -- Utrecht 1993: Knag/Faculteit Ruimtelijke Wetenschappen Universiteit Utrecht, ca 160 pp. ISBN 90-6809-176-X Dn 32,00

164 J R RITSEMA v ECK, Analyse van transportnetwerken in GIS voor sociaal-geografisch onderzoek. - Utrecht 1993: Knag/Faculteit Ruimtelijke Wetenschappen Universiteit Utrecht. ca 224 pp. ISBN 90-6809-177-8 Dn 35,00

165 P VAESSEN, Small business growth in contrasting environments - Utrecht/Nijmegen 1993: Knag/Faculteit Beleidswetenschappen Katholieke Universiteit Nijmegen. ca 224 pp. ISBN 90-6809-178-6 Df 35,00

Publications of this series can be ordered from kNAG / NETHERLANDS GeOGRAPHICAL STUdiES, P.O. Box 80123, 3508 TC Utrecht, The Netherlands (Fax +31 30 535523). Prices include packing and postage by surface mail. Orders should be prepaid, with cheques made payable to "Netherlands Geographical Studies". Please ensure that all banking charges are prepaid. Alternatively, American Express, Eurocard, Access, MasterCard, BankAmericard and Visa credit cards are accepted (quote card number and expiry date with your signed order). 



